\title{
THE END OF THE REGULAR COIN SUPPLY IN THE CROATIAN PART OF THE DANUBE LIMES
}

\author{
Katarina Lukić \\ Kralja Zvonimira \\ HR - 10000 Zagreb \\ katarina280793@yahoo.co.uk
}

UDK / UDC: 904:737.1(497.54-37 Podunavlje)"652" Izvorni znanstveni rad/Original scientific paper https://doi.org/10.52064/vamz.54.1.2

This paper analyses, compares and presents numismatic data gathered by examination of Late Roman coin material, from both the display and study collections of the Archaeological Museum in Zagreb, discovered in the area of two Roman castella in the Croatian part of the Danube limes: Teutoburgium (Dalj) and Cornacum (Sotin). This data has been used for constructing the chronology of the coin supply to, and annual coin circula-

Key words:

Late Antiquity, Danube limes, Pannonia Secunda, coin supply, Roman military tion intensity at, the sites in the set time frame - i.e. from the Valentinian dynasty until the latest late-imperial Roman coin - in order to try to establish the end of the regular coin supply, and thus contribute to better understanding of the socio-economic and administrative changes in the aforesaid area in the late $4^{\text {th }}$ century. All determined coins are catalogued in detail, while data analysis is presented in charts and tables.

\section{Introduction}

Castellum Teutoburgium and Cornacum were constituents of a complex frontier system of the Roman Empire known as limes. ${ }^{1}$ The Late Antiquity period of the Pannonian portion of the Danube limes region is especially interesting for research due to various dire events which eventually affected the survival of the western half of the Empire. For the Croatian part of the limes, exactly $188 \mathrm{~km}$ from modern-day Batina Skela (Ad Militare) in the $\mathrm{N}$ to llok (Cuccium) in the $\mathrm{S}^{2}$ (Fig. 1), the Battle of Mursa (Osijek) in 351 between emperor Constantius II and usurper Magnentius, as well as more frequent incursions of various ethnic groups into the Empire, can be pinpointed as such occurrences. ${ }^{3}$ These and other external and internal nuisances that affected the Empire throughout the $4^{\text {th }}$ century and into the beginning of the $5^{\text {th }}$ ultimately led to administrative collapse and the end of the Western Roman authority over the territory. ${ }^{4}$

This work will attempt, from a numismatic perspective, to contribute to better understanding of this complex topic by trying to determine the last regular coin supply, i.e. its end, to the two aforementioned Roman forts. ${ }^{5}$

\footnotetext{
$1 \quad$ Cf. Isaac 1988; Visy 2005, 213; Sanader 2010, 225. For history of research on limes in Croatia see Vukmanić 2009, 31-32

2 Sanader 2003,$135 ; 2010,225$. The area administratively belonged to Pannonia Secunda in the $4^{\text {th }} \mathrm{C}$.
}

3 Mócsy 1974, 286; Pinterović 2014, 191; e.g. "Sarmatian” incursions into Pannonia Secunda in 374, 380 and, possibly, 385, as well as attacks of Gothic groups in 380 (Mócsy 1974, 286; Kovács 2016, 582-586). About the re-examination of Sarmatian ethnicity see Dan 2017

\footnotetext{
$4 \quad$ Mócsy 1974, 352.

I would like to thank T. Bilić, M. Nađ and I. Radman-Livaja of the Archaeological Museum in Zagreb for their guidance and help in the making of this
} work. 


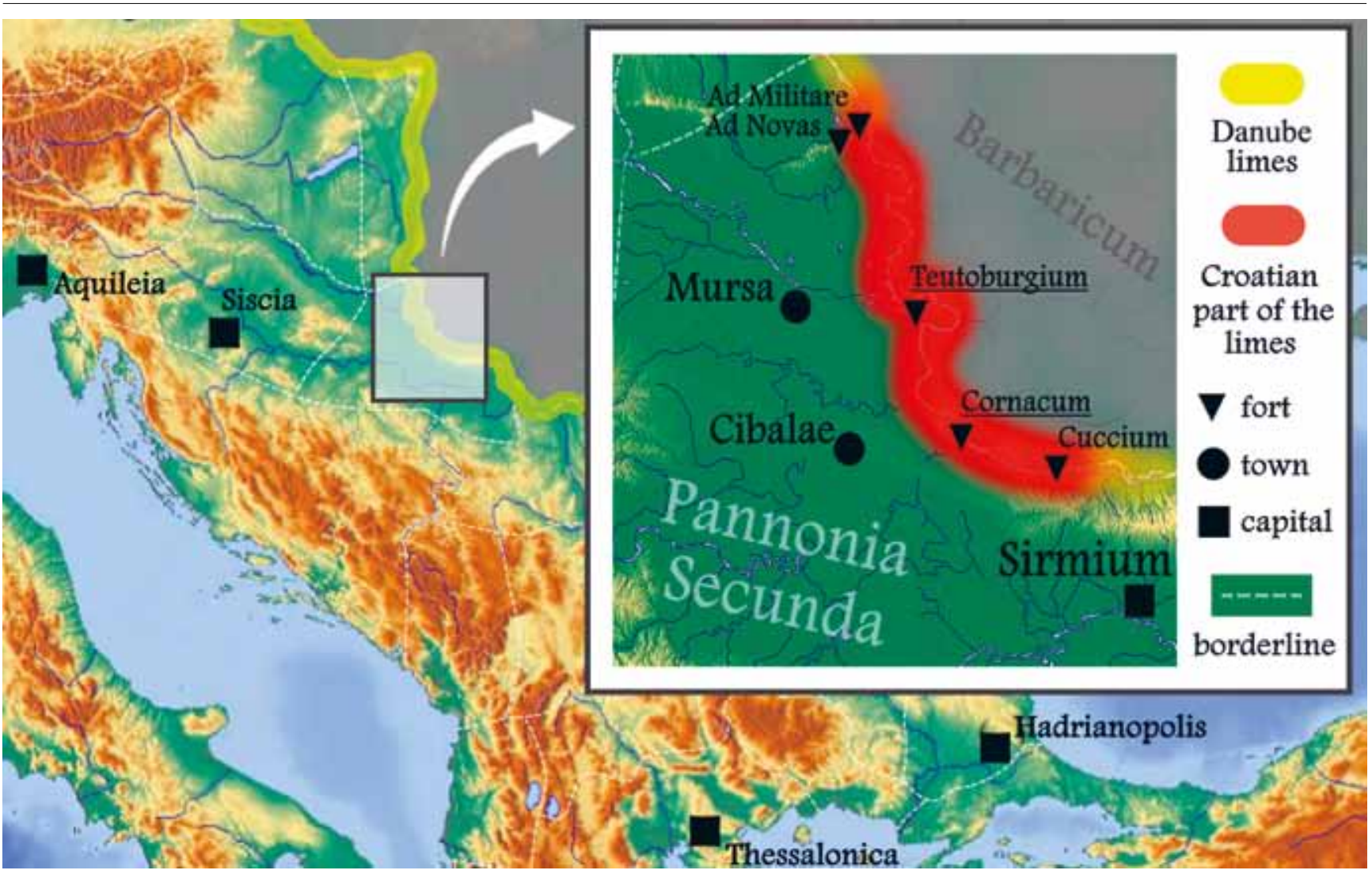

FIGURE 1. Position of the castella (underlined) in the Croatian part of the Danube limes (made by K. Lukić). ${ }^{6}$

\section{Money under the Valentinian Dynasty}

In view of the historic events in the area observed and the aim of this work, among the overall Late Roman numismatic material found in the area of the castella only those coins from the valentinian dynasty onwards have been examined.

On coming to power, emperors Valentinian I and Valens installed reforms $(365-368)$ to restore the purity of bullion and lower prices after the unsteadiness of the monetary system in the $1^{\text {st }}$ half of the $4^{\text {th }}$ century. ${ }^{7}$ Another important change, introduced in 368 , regarding the production of precious metal coins, was the shifting of their manufacture to comitatensian mints that operated mostly at the emperor's residence. ${ }^{8}$ These changes were indirectly prompted by Valentinian I's overhaul of social grading policy with an increasing amount of mobility within the hierarchy (which was especially beneficial for militares). ${ }^{9}$ Moreover, with the edict of 371 , the brother emperors withdrew from circulation all bronze-silver alloy denominations (dichoneutum) and prohibited trade with them, as well as their possession, while silver from withdrawn coins was recycled for production of argentei. The edict of 371 ended the 4-century-long tradition of minting billon coinage in the Empire, and as a consequence solely basemetal coinage became significant once again in an overall golddominated system. ${ }^{10}$ The main bronze denomination under the early Valentinian rule was $Æ 3$ nummus $(2.25 \mathrm{~g}$ ) of the GLORIA ROMANORVM and SECVRITAS REIPVBLICAE type. However, the bronze coinage underwent another reform in 379 when, after his elevation to senior Augustus, Gratian introduced three new denominations. These nummi emulated coins introduced in a
6 The map includes only archaeologically and historically confirmed fort locations. See Sanader 2003.

Harl 1996, 172; Valentinian I decreed that all taxes should be paid in pure gold and delivered to the imperial treasury (scrinium aureae massae) as bullion. This required regular melting of coins. (Cod. Th. 10, 24, 3; Hendy 1985, 320); coins with improved fineness were marked as $\mathrm{OB}$ (obryzum aurum, pure gold) and PS (pusulatum, pure silver) respectively (Moorhead 2012, 602, 609).

8 This resulted in the appearance of the $\mathrm{COMOB}$ mintmark on gold coins (RIC 10, 23-25; Moorhead 2012, 603).
Re-grading required increase in payment which was then levelled by purified gold coins. This endorsed commutation from payment in kind to cash on a wider scale, enabling profiteering by certain groups within the bureaucracy (Banaji 2002, 51, 216).

$10 \mathrm{Harl}$ 1996, 172; Banaji 2002, 87. R. Reece argues that bronze coinage might have had only commercial purpose and circulated only as token value depending on the validation of bullion, whereas precious-metal coins had more of a fiscal value. According to Reece, the Roman Empire distributed its expenditures solely in gold and silver coinage, making the payees first exchange them for bronze denominations at money-changers, thus ensuring the return of precious metals into the Empire's treasury (Reece 2003, 142). About the model, its limitations and other possible interpretations see Reece 1984; for silver coinage see Moorhead 2012, 609-611. 
reform of 348. The largest, $Æ 2(5.25 \mathrm{~g})$, was similar to the withdrawn maiorina and mainly struck with reverse type REPARATIO REIPVB, while the smallest denomination, nummus minimus ( 1.5 g), was in $Æ 4$ flan size and minted with reverse types VOTA, SALVS REIPVBLICAE and VICTORIA AVGG ${ }^{11}$ The main denomination, corresponding to centenionalis, was $Æ 3(2.45 \mathrm{~g})$ with prevailing reverse type CONCORDIA AVGG. This tripartite bronze system continued until the emperor Theodosius I, between 388 and 392, stopped the minting of $Æ 2$ and reduced the production of $Æ 3$ in western mints. Production of $Æ 4$ nummi resumed, albeit of a reduced standard. ${ }^{12}$

Gold, apart from its mercantile value, proved to be an adequate diplomatic asset (in the form of a centaurim - 100 librae of gold) used for controlling the situation along the borders and for paying off foederati. Such payments drastically increased at the end of the $4^{\text {th }}$ century, and general growing demand for gold ultimately led to the downfall of the bronze-based monetary system in the west. ${ }^{13}$ The main gold denomination in the Valentinian period was still solidus ( $4.48 \mathrm{~g}$ ), whilst in 383 Maximus introduced, and, beginning in 388 , Theodosius I continued minting, the tremissis ( $1.5 \mathrm{~g}$ ) as the smallest gold coin. ${ }^{14}$

The monetary circulation in the Roman Empire probably began to decline as a consequence of the socio-economic state that the Empire found itself in during the last third of the $4^{\text {th }}$ century, with the aftermath of the Battle of Hadrianopolis in 378 usually reckoned as a starting point. Increased incursions into imperial territory and other menaces in the Empire must have had a negative effect on the economy in the affected areas, particularly rural, which resulted in the decrease of money circulation. ${ }^{15}$ The numismatic data from Pannonia Valeria demonstrates the aforesaid, implying that termination of the regular coin supply generally followed after $378 .{ }^{16}$ The final coin supply of the Pannonian provinces north of the Drava (Prima, Valeria) is represented by $\notin 4$ coins of the SALVS REIPVBLICAE type. This probably occurred around 395 or 400 , while the coin data from certain inland towns in Pannonia Savia and Secunda suggests unobstructed supply and circulation, albeit of a rather low intensity, continuing into the first half of the $5^{\text {th }}$ century. ${ }^{17}$ Regular coin supply in the neighbouring provinces on the Empire's border, in Noricum Ripense and Raetia Secunda (Vindelica) in the west, likewise ended around 400 with $Æ 4$ SALVS REIPVBLICAE, ${ }^{18}$ whereas coin finds from Moesia Prima and Dacia Ripensis to the east suggest the supply continued until the $1^{\text {st }}$ half of the $5^{\text {th }}$ century. ${ }^{19}$

\section{History of research}

\section{Dalj (Teutoburgium)}

The modern-day village of Dalj developed on the right bank of the Danube, $19 \mathrm{~km}$ NW of the town of Vukovar, in the vicinity of the Drava's confluence with the Danube. There have been no systematic archaeological excavations in the area, but the results of trial trenches and the variety of surface stray finds of Roman provenience, as well as referrals in contemporary sources, attest to the existence of castellum Teutoburgium.$^{20}$ Alongside written sources, epigraphic inscriptions found in the area correspond with numerous finds attributed to military horse equipment, suggesting that mainly cavalry units were stationed at the fort. ${ }^{21}$ Unfortunately, the abrasive forces of modern construction and the River Danube have almost completely disintegrated the Roman architecture, complicating the research of Teutoburgium even more. ${ }^{22}$ From the abovementioned finds, only certain items of military and sepulchral purpose have been published. ${ }^{23}$ Roman coins have not been examined hitherto.

\begin{abstract}
11 RIC 9, 31; Harl 1996, 175
12 In 395 Emperor Honorius prohibited the use and possession of $Æ 2$ coins, and consequentially $\mathbb{E} 4$ nummi became prime currency in the western part of the Empire. $\mathbb{E} 2$ is mentioned in the 395 edict as decargirus, i.e. "silvered tenth", suggesting it might at some point have been worth 10 nummi and, as such, a possible prototype for Byzantine decumania, formed in the late $5^{\text {th }}$ century. The E 3-sized nummus is referred to in the edict as centenionalis (Harl 1996, 174).

13 Harl 1996, 176; Reece 2003, 147. According to S. Moorhead, the purpose of gold in civilian parts of the Empire might have varied from military frontier zones, where it had been used more for paying donatives, gifts and tributes (Moorhead 2012, 606-608); the state's traditional process of integrating foreigners into the socio-economic life of the Empire (receptio) became inefficient with the influx of Goths in the last third of the $4^{\text {th }}$ century; and, after the Empire sanctioned a foedus with certain Goths in 382, other ethnic groups (including Goths) became increasingly encouraged to demand similar agreements (Modéran 2008, 221); see Burns 1994, 12-15, 108
\end{abstract}

14 RIC 9, 26; Moorhead 2012, 604; the tremissis would become the prime gold denomination in the west after the collapse of the Western Empire (Harl 1996, 175, 483)

15 Burns 1994, 42; Harl 1996, 175-176; various Gothic groups, having settled on imperial territory in the last third of the $4^{\text {th }}$ century, certainly contributed to the disruption of monetary circulation, because they had not, at least not completely, integrated into the socio-economic life of the Empire (Mócsy 1975, 343344). Another factor that destabilized the economy and social order in Pannonian provinces was the oppressive tax policy of Probus, PP of Illyricum (Bratož 2011, 590).

16 Burger 1981, 153, 185, 192; RAMMU 1, 3

\footnotetext{
17 Alföldi 1924, 23-35; 1926, 58; FMRÖ 1/2; Kos 1986, 224; see Nađ 2012; apart from coin finds, an indication that administrative organization in the towns of Pannonia Secunda was still present to a certain degree at the end of the $4^{\text {th }}$ cen tury can be found in the inscription of Flavius Lupus ( $A E$ 1968, $113=1998,369=$ 2001, 610; Kovács 2016, 586).

18 FMRD 1, 3; Kos 1986, 223; 2019, 117-119.

19 Rankov Kondić 2013, 44, 54; Vojvoda, Mrđić 2017, 69, 341; for the circulation in the Serbian part of the limes see Vojvoda, Redžić 2018

20 Bulat 1974, 85; Sanader 2003, 141; Teutoburgium (Ptol. Geog. 2, 15, 3); Teu tiburgio (It. Ant. 243, 4); Tittoburgo (TP 5A2, Talbert 1638); 4/5 $5^{\text {th }}$ century Notitia Dignitatum mentions Castellum Teutiborgio, i.e. Teutibarcio, garrisoned with cavalry units equites promoti and cuneus equitum Dalmatarum, as well as de tachments (Vexillationes) of the legio VI Herculia (Not. Dign. [occ.] 32, 4, 11, 23, 30, 47).

21 Radman-Livaja 2012, 176; for references on epigraphic sources see Sanader 2003, 141; Radman-Livaja 2012, 176, n. 144.

22 Sanader 2010, 230.

23 See Bulat 1977; Radman-Livaja 2005
} 


\section{Sotin (Cornacum)}

Sotin is a village located $29 \mathrm{~km}$ SW of Dalj and situated on the right bank of the Danube. ${ }^{24}$ Systematic archaeological excavations have not yet been conducted in the area, but six campaigns of trail excavations (2008-2013) by the Institute of Archaeology and Vukovar Municipal Museum have determined a cultural continuity from Prehistory to the Early Medieval period. Regarding the Roman period, $S$ of the presumed position of castellum Cornacum (Popino brdo), a double ditch (fossatum) of an auxiliary military camp has been discovered, defining the camp's northern and eastern limit. Moreover, peripheral parts of a settlement, possibly an area of production, were discovered between the military camp and the fort, as well as presupposed traces of a road, running NW-SE and W-E respectively, with $2^{\text {nd }}-4^{\text {th }}$ century cremation and skeletal burials along them. It was concluded that the modern village partially overlies a Roman settlement which it must have destroyed, and which is still eroding the existing strata. Nonetheless, on the premise of excavated artefacts, two preliminary layers dating from $2^{\text {nd }}-4^{\text {th }}$ century have been established. In addition, SE of the Roman settlement, i.e. on the Srednje polje position, remains of a settlement with ceramic material dating to the Migration Period have been found..$^{25}$

Apart from surviving features, certain literary sources and various single finds corroborate the existence of the fort and an accompanying settlement. ${ }^{26}$ Among the abovementioned finds, Roman coinage has been found in abundance; ${ }^{27}$ and, based on the numismatic-topographical analysis of part of the material, M. Ilkić has provided some conclusions on the settlement history, the extent and the end of Cornacum. ${ }^{28}$ On the basis of the latest coin type, M. Ilkic sets the end of the occupancy of the majority of the settlement at the end of Valentinian I's rule, or under Valens's rule, ${ }^{29}$ whereas continuous occupation after 378 can only be recognized at the supposed location of the fort (Popino brdo). Regarding the fact that $Æ 4$ SALVS REIPVBLICAE coins, minted between 388 and 403, are the latest-dated numismatic finds, and that there are no coins attributed to emperor Honorius, the author has concluded they might represent the final military payment received before summer 392, connecting military departure with the end of the habitation of the area. ${ }^{30}$
On the other hand, A. Alföldi, on the basis of his research of the systematic collection of the Archaeological Museum in Zagreb, assumed the end of coin supply to the area to fall in $387-388 .^{31}$

\section{Analysis}

In total, 618 Late Roman coins, issued from the Valentinian dynasty onwards, and found on the territory of modern-day Dalj and Sotin, have been examined in the collections of the Archaeological Museum in Zagreb. The majority - 436 coins were found in the Sotin area, whilst 182 have come from the area of Dalj. All except cat. no. 249 and cat no. 475 are Late Roman bronze denominations, among which $Æ 3$ nummi are represented in largest numbers (Dalj $95 \%$, Sotin $87 \%$ ), followed by $Æ 4$ (Dalj $3 \%$, Sotin $11 \%$ ) and $Æ 2$ (both $2 \%$ ). Cat. no. 249 is a RESTITVTOR REIPVBLICA solidus struck under Valentinian I in Antiochia between 364 and 367, while cat. no. 475 is a GLORIA ROMANORVM solidus of emperor Valens, minted in Antiochia between 367 and 375 . Both solidi were found in the Sotin area, and the latter has already been published. ${ }^{32}$ Furthermore, the sites have similar mint distribution, with the Siscia mint clearly dominating in the period under examination. Analysis has shown that $68 \%$ of all coinage from Dalj, and $64 \%$ from Sotin, had been struck in Siscian officinae. The mints of Aquileia (Dalj $10 \%$, Sotin $8 \%$ ), Thessalonica (Dalj $9 \%$, Sotin $16 \%$ ) and Roma (Dalj $6 \%$, Sotin $4 \%$ are also represented in considerable number in comparison to other recognized mints. The aforesaid data is analogous with that from other Pannonian military and civilian sites connected with the limes. ${ }^{33}$ Therefore both forts exhibit the mint distribution characteristic of the wider limes area in the last third of the $4^{\text {th }}$ century34 (Fig. 2).

On the basis of numismatic identification of 618 items, a representative sample of 534 coins with distinguishable time of issue and other elements needed for further analysis was arrived at. The coins examined belong to the category of single finds recovered outside archaeological context, and are therefore only suitable for establishing the coin supply at the sites..$^{35}$ In other words, a comparison of representation of coins of certain emissions has been made in order to ascertain the intensity of monetary circulation in different time periods at the localities in question. ${ }^{36}$ The
24 Presumably, the contemporary road from Dalj to Sotin follows the same direction as did the Roman road that once connected the two castella, skirting the Danube's right bank (Bulat 1969, 43).

25 Ložnjak Dizdar, Hutinec (eds.) 2010; Ložnjak Dizdar, Hutinec 2011, 9-10; 2013, 9; 2014, 9 .

26 Cornacum (Ptol. Geog. 2, 15, 3) Cornaco (It. Ant. 243, 3, TP 5A2, Talbert 1639, Not. Dign. [occ.] 32, 12), n.b. Cornacu (Not. Dign. [occ.] 32, 3); on the finds see Sanader 2003, 141; see also Ilkić 2005; 2006; 2008a; 2008b; 2009a; 2009b; Ložnjak Dizdar, Hutinec (eds.) 2010; Radman-Livaja 2012, 176-177.

27 The majority of these are stray finds, kept in private collections and in several museums (Mirnik 1999, 226; Ilkić 2008a, 51-52); aside from the single finds, an Early Imperial coin hoard has been found as well (Brunšmid 1911, 251277).

28 Ilkić 2003, Table 1-2; 2008a, 52; I would like to thank M. Ilkić of the University of Zadar for allowing me to use essential data from his unpublished PhD thesis.

29 Ilkić 2008a, 55, PI. VI, 1-3

$30 \quad$ Ilkić 2003, 128, Map 7; 2008a, 55, PI. VI, 8-10; according to Notitia, cavalry units: equites Dalmatae, cuneus equitum scutariorum and equites promoti were stationed in Cornacum in the $4^{\text {th }}$ century (Not. Dign. [occ.] 32, 22, 31).
31 He arrived at this conclusion on the basis of two coins: inv. no. C 25241 (cat. no. 612) and inv. no. D 105 (cat. no. 614) (Alf Idi 1924, 27).

32 Demo (ed.) 1994, 127, cat. no. 183; gold coins have not been included in the statistical analysis and data interpretation because the number of gold coins studied in the sample is inadequate for further analysis. Likewise, on the assumption that R. Reece's above model of bullion cycle is correct, gold coins are suitable not for studies of coin supply, but rather for studies of coins as struck (Reece 2003, 143).

33 Pannonia Valeria (Burger 1981, 194-198); Carnuntum (MIR 36, 43, 44, 181, Table 2.33; FMRÖ 3/2)

3421 coins from Dalj and 46 from Sotin have undeterminable mint marks and therefore were omitted from the analysis.

35 Reece 2003, 141-165; 2016, 182; Bilić 2018, 272-273.

36 The possibility that the time of a coin's introduction into the circulation does not necessarily need to correspond with that of its minting should be taken into consideration when conducting this type of analysis (Vučić 2013, 226). 


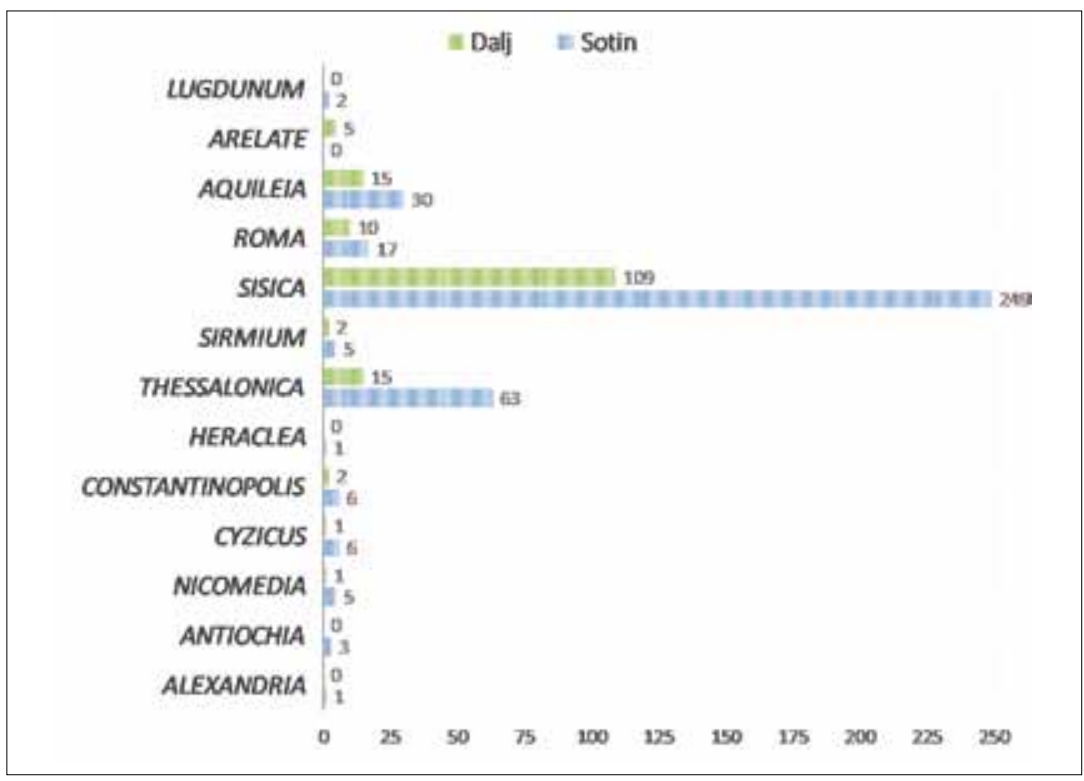

FIGURE 2. Mint distribution at Teutoburgium and Cornacum (made by K. Lukić)

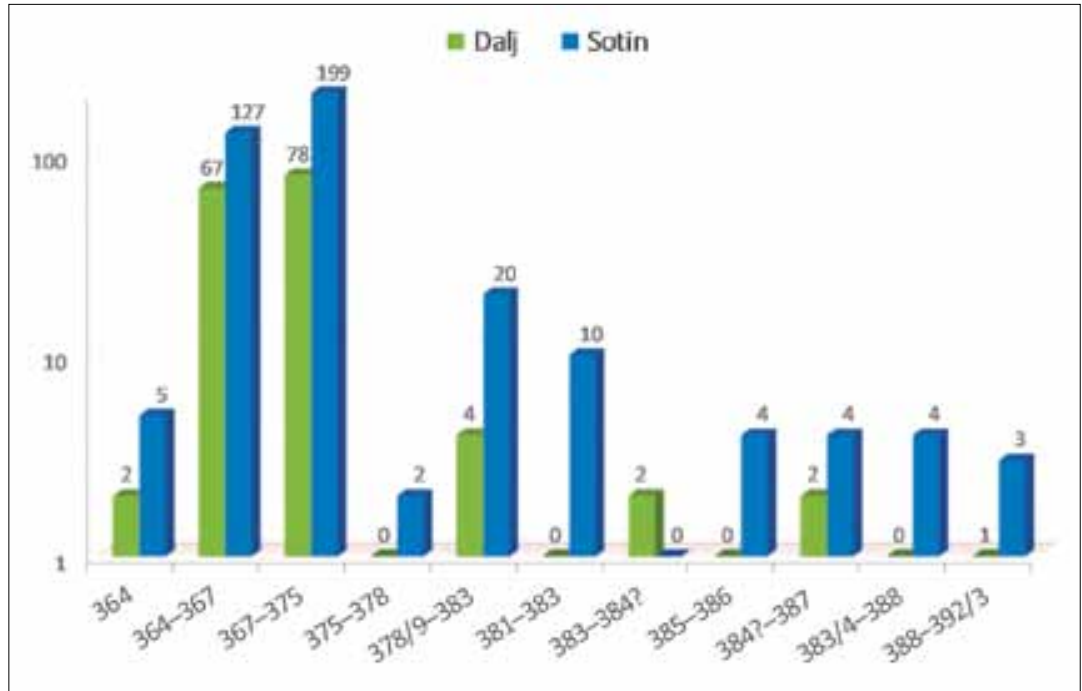

FIGURE 3. Comparison of the coin supply at Teutoburgium and Cornacum (made by K. Lukić).

numismatic analysis of the representative sample has recorded seven different coin emissions between 364 and 393 on 156 coins from Dalj, whilst among the 378 coins from Sotin it was possible to define 10 coin emissions from 364 to 392 (Fig. 3)

Given that certain emissions in the sample are to some extent overlapping, it was possible to merge them into six general time periods in order to present more clearly the chronological progression of the intensity of coin circulation at the two sites. This data has been inserted into the "annual circulation intensity per emission" calculation formula (i.e. index) ${ }^{37}$ and, by dividing the number of coins from a certain time period by the number of years in the period and the number of coins from all compared samples, the annual circulation intensity was calculated and presented with its fluctuation value (Table 1)..$^{38}$
In the calculated intensity values, an analogous progression between 364 and 383 can be seen at both sites (Fig. 4). The period between 364 and 367 , represented by $Æ 3$ nummi of emperors Valentinian I and Valens of types GLORIA ROMANORVM (cat. nos 3-12, 29-46, 69-71, 188-218 and 252-280), SECVRITAS REIPVBLICAE (cat. nos 1, 13-24, 47-67, 72, 184, 219-248 and 281314), RESTITVTOR REIPVBLICA (cat. nos 25-27, 68, 183, 187 and 250-251) and VOTA (cat. nos 2 and 185-186), displays the highest value of coin circulation intensity, while the first observed fluctuation in value happened in the following period. The analysis indicates considerably decreased coin circulation intensity between 367 and 375 in comparison to the previous period. The $Æ 3$ GLORIA ROMANORVM (cat. nos 91-103, 119-132, 162-168, 356-400, 452-474 and 518-547) and SECVRITAS REIPVBLICAE (cat. nos 105$118,134-161,169-170,401-451,476-517$ and 549-556) of emperors 


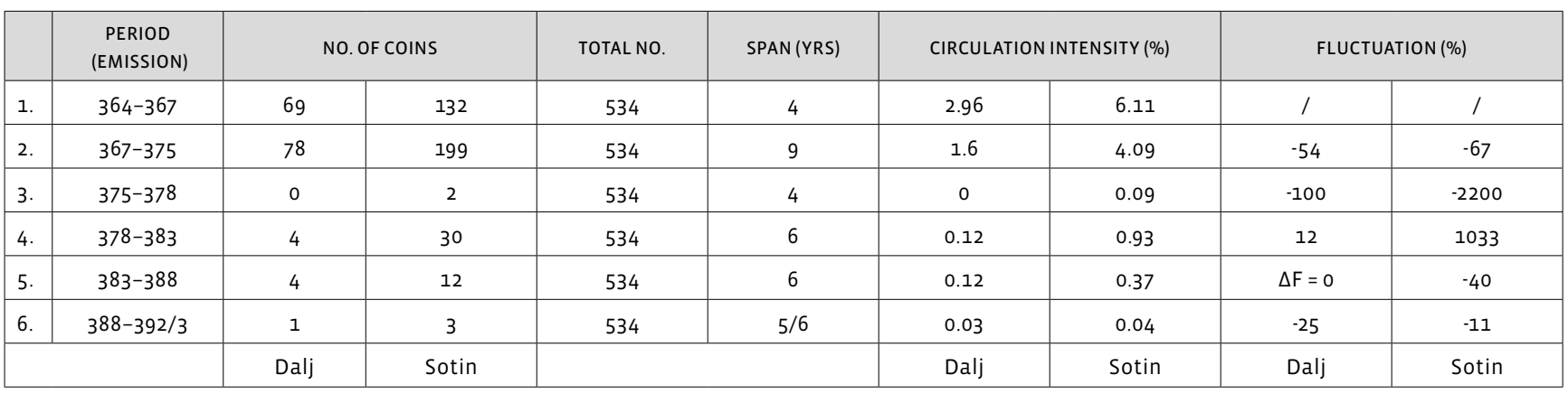

TABLE 1. Annual circulation intensity per period in Teutoburgium and Cornacum between 364 and 392/3 (made by K. Lukić).

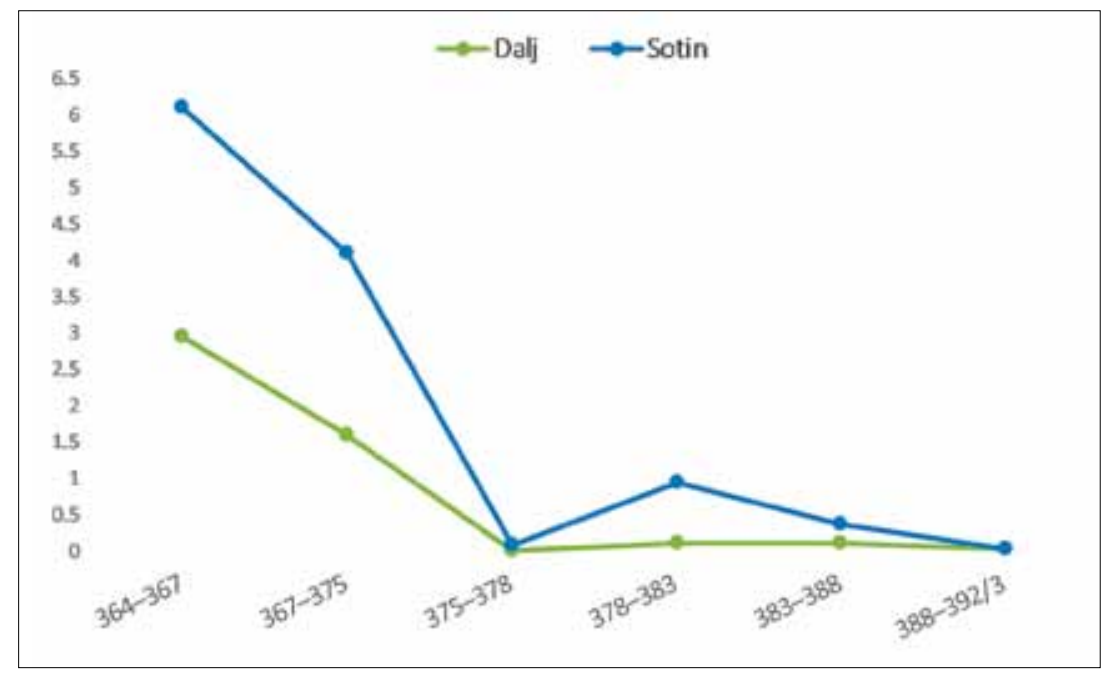

FIGURE 4. Comparison of the coin circulation in tensity at Teutoburgium and Cornacum (made by K. Lukić).
Valentinian I, Valens and Gratian belong to the abovementioned period. An increase in the reduction of coin circulation intensity continued at both sites during the period 375-378. In the sample, only two $Æ$ z GLORIA ROMANORVM nummi of Valens (cat. no. 557), and Gratian (cat. no. 558), issued at Thessalonica and found in the Sotin area, can be dated to this period. ${ }^{39}$ If the bronze coins are to be interpreted according to Reece as military payments (regardless whether they represented an actual form of pay or small change), then the decrease in the circulation intensity could be seen as an indirect result of reduced military activity in the area, i.e. the presence of a smaller number of soldiers. $4^{0}$ This interpretation raises a question: whether such reduction is then related to conditions demanding lesser military presence in the area or to diminished military capacity. Although no firm conclusion on the matter can be drawn without systematic archaeological excavations being carried out, literary sources can provide some information. Taking into consideration the report of a contem- porary, Ammianus Marcellinus, of successful campaigns of the Pannonian troops under Frigeridus against Goths and Taifali in the neighbouring provinces at the beginning of the Gothic war (376-382), it seems possible to suppose that Pannonia was rather peaceful around $375-378,{ }^{41}$ and therefore in no need of great military presence on its borders. The fact that the young heir of the west, Valentinian II, resided in Sirmium at that time supports the idea that the borders of Pannonia Secunda were not threatened. ${ }^{42}$ Hence, these accounts might imply that the low coin circulation intensity in the area was due to the troops who previously garrisoned the two castella being stationed and paid, and spending their money, somewhere else, where their presence was needed, around $375-378 .{ }^{43} \mathrm{~A}$ similar conclusion might be applied to preceding emissions, connecting the more intense coin circulation with Valentinian's construction campaigns along the frontier, as well as with the conflict with the Sarmatians and Quadi in $374-375.44$
39 Although it was excluded from the analysis due to the fact that it was not possible to precisely identify the issue, Valens's E 3 SECVRITAS REIPVBLICAE from Dalj (cat. no 171) might be associated with this period.

40 Reece 1982, 498; 2003, 142-143; Moorhead 2012, 615. See also Callu 1980, 105-106; Duncan 1993
41 Amm. Marc., 31, 7, 3; 9, 1-4; 10, 21; Kovács 2016, 577.

42 Mócsy 174, 295; Paul. Mil. v. Ambr., 11, 1; Kovács 2016, 581

43 Another factor affecting the coin circulation at military sites is the accompanying settlements (vici) that were usually forming in the vicinity. Although military dependants probably followed the troops on their campaigns, some of the populace must have remained in the vicus, and the fort would certainly not have been left unattended. Some civilians lived inside the forts, as well (Hanson 2005, 304-305).

Mócsy 1974, 291-295; Visy 2003, 46; Amm. Marc. 29, 6, 6, 13; perhaps the coin finds from Sotin cat. no. 356 and cat. no. 476, minted at Lugdunum, could be associated with the conflict (Amm. Marc. 29, 6, 16); see also CTh. 15, 1, 13. 
After 378, the circulation intensity dwindled at both sites in comparison to prior periods, with the last recognized increase being a modest one between 378 and 383 . This period is represented in the sample by coinage of $Æ 2$ REPARATIO REIPVB (cat. nos 172-173, 560-561 and 576-578), Æ 3 CONCORDIA AVGGG (cat. nos 579-580 and 586) and $Æ 4$ VOTA (cat. nos 174-175, 562-571, 581-582, 587593 and 596) and VICTORIA AVGG (cat no. 559), issued, mainly in Siscia, for the emperors Gratian, Theodosius I and Valentinian II. Said period is marked by the culmination of the Gothic War and 'barbarian' plundering of the imperial territory after Valens's defeat at Hadrianopolis, as well as a general migration of various ethnic groups towards the Empire, reported by Ammianus. ${ }^{45}$ In 380, Gothic groups led by Alatheus and Safrax raided Pannonia Secunda in a counterattack, during which Mursa (Osijek) was pillaged..$^{46}$ In addition, there is the possibility of associating coin hoards with material dated up to 378 with the Gothic War. Such hoards have been found throughout the Pannonian provinces, attesting that the region was in peril. ${ }^{47}$ The aforesaid situation must have demanded reinforcing the number of soldiers in the affected area, which might be related to the recorded rise of the circulation intensity at both sites. Still, generally low intensity in the castella after 378 might be due to the attenuated capacity to garrison them with regulars and/or the loss of their strategic, defensive importance, especially after the foedus of 382 with some Gothic groups. $4^{8}$ The results of the study of coinage from the Sotin area correspond with M. Ilkić's dating of the end of habitation in the majority of the auxiliary vici; hence, the drastic reduction in coin circulation intensity in Cornacum after 378 could also be connected with the abandonment of the settlement due to these circumstances (although it could possibly already have been unoccupied from 375). 49

The sites display different chronological progressions after 383. The data from Dalj exhibit no change in coin circulation intensity between 383 and 388 in comparison to the previous period (Figs 3-4). The nummi of GLORIA ROMANORVM type, of $Æ 2$ (cat. no. 177) and $Æ 3$ (cat. no. 180) flan size, and $Æ 4$ VICTORIA AVGG (cat. no. 181) of emperor Theodosius I, as well as $\mathbb{E} 2$ SALVS REIPVBLICAE of his wife Flaccilla (cat. no. 176), all minted in Siscia, belong precisely to this period. However, after 388 the coin circulation intensity decreased again. The latest recorded coin supply to Teutoburgium occurred between 388 and 393 and is represented by $Æ 4$ SALVS REIPVBLICAE of emperor Arcadius, coined in Thessalonica under Theodosius (cat. no. 182). The aforesaid circumstances suggest that, between 383 and 393, troops, perhaps cavalry units, loyal to Theodosius I, could have been stationed in Teutoburgium, and could probably have been the last regular garrison in the fort. The results of this study suggest that the official coin supply to the area of Teutoburgium might have ended soon after 393 (Fig. 4).

On the other hand, the data from the Sotin area display continual decrease in the 383-388 period, with a majority of coinage issued at the Siscia mint, after its restoration to Valentinian II, 50 and from Thessalonica (Fig. 3). The $Æ 4$ VICTORIA AVGGG of emperors Valentinian II (cat. nos 604-607), Theodosius I (cat. nos 598 and 608-609) and Arcadius (cat. nos 611 and 614), as well as Arcadius's $Æ$ 3 GLORIA ROMANORVM (cat. no. 610) and Theodosius I's $Æ$ 3 VIRTVS AVGGG (cat. nos 612-613) are all dated to this period. ${ }^{51}$ The higher concentration of Siscian issues minted after 384 in the fort might possibly be put in the context of internal political turmoil in the Empire, provoked by Maximus's usurpation. The latest recognized supply to Cornacum occurred between 388 and 392 and is represented by $Æ 4$ SALVS REIPVBLICAE of emperors Valentinian II (cat. nos 616-617) and Arcadius (cat. no. 618). Thus, the regular coin supply to Cornacum, according to the date of the latest recorded emission in this analysis, ceased $392^{52}$ (Fig. 4). Cat. no. 616 was minted in Aquileia, and cat. no. 617 in Roma, whereas cat. no. 618 comes from the eastern mint in Cyzicus. Considering that, after Maximus's death in 388, Italian mints formerly of Valentinian II came under Theodosius I's con trol, ${ }^{53}$ and therefore all the represented mints in this period have been under his control, it can be supposed that the possibly last regular troops in the fort had likewise been under his command. ${ }^{54}$

Although the number of coins in the last detected emissions from the analysed sample does not suffice it to safely presume that the latest regular coin supply to both localities happened sometime between 388 and 393, these results can be seen as a starting point for further and broader numismatic analysis of the Croatian part of the Danube region. If these numismatic finds are, in fact, military payments of the garrisons that had been stationed at the sites, the simultaneity of their latest coin supply, that is salaries, might serve as a criterion for constructing the chronological time frame for the end of the regular military pres ence in, and consequently defensive significance of, this part of the limes (taking into account the possibility that money could have entered into the circulation slightly later). Moreover, dating obtained in the analysis coincides with literary sources referring

\footnotetext{
45 Amm. Marc. 31, 4, 2; 16, 7; Mócsy 174, 340-341.

46 Kovács 2016, 582, 584.

$47 \quad$ Mócsy 1974, 294; Torbágy 2000, 43-45, 52, Table 1; approximately 20 hoards with bronze coins struck by 378 for Valentinian I, Valens and Gratian have been detected on the territory of Sotin (IIkić 2008a, 55); for contemporary hoard finds in the surrounding area see Nađ 2012, 409, 412

48 Mócsy 1974, 346; on the foederati see Kovács 2016, 582-586.

49 Cf. Ilkić 2008a, 55; the phenomenon of abandoning the auxiliary vici and the retreat of the remaining population to forts, starting from the 360 , has been archaeologically recorded along the limes in Pannonia Valeria (Kovács 2003, 33).
}

50 In 383 the Siscian mint temporarily fell under Theodosius I's authority following his occupation of Valentinian Il's territory as a precautionary measure against Maximus (RIC 9, 22).

51 A. Alföldi dated cat. no. 604 and cat no. 610 to 387-388 (Alföldi 1924, 26).

52 The results attained correspond to the results of M. Ilkićs analysis and are coherent with the author's two-phased dating of the end of life in Cornacum (cf. Ilkić 2008, 55).

53 RIC 9, 26, 167

54 The fact that, at both castella, only Æ 4 of the SALVS REIPVBLICAE type have been identified so far might suggest that, in the late 80's and early 9o's of the $4^{\text {th }}$ century, the area still operated as part of the western monetary system, but under Theodosius's authority (Moorhead 2012, 617). 
to the loss of Pannonian frontier provinces. ${ }^{55}$ The dramatic narrative of contemporaries about total destruction starting around 375-378 and lamentation on the loss is surely an exaggeration originating in certain authors' biased agendas, perspectives and writing styles, referring to events which probably happened only after Theodosius's death, i.e. after $395^{.66}$

That said, even if it is possible to find correlation between coin circulation intensity at the sites and military activity in the area, as I hope I have demonstrated above, following R. Reece, it is in fact highly speculative to try to see a particular event as a sole and definitive cause of the fluctuations in circulation (since correlation does not imply causation). At the same time, it should be kept in mind that there were other forms of pay present apart from in-cash (see below); therefore, the parallels regarding coin circulation intensity adduced here are just a general outline in need of further exploration endorsed by systematic archaeological excavations.

It must be emphasized that the dating suggested for the end of the coin supply in the area studied should not be seen as implying the collapse of the administrative services and economic life in the frontier zone of Pannonia Secunda - merely its degradation. The Roman economy, especially rural, was probably never fully monetized, and barter was certainly present alongside coinage and used as an acceptable means of payment during money shortages, ${ }^{57}$ although cash payments, especially in gold, became a more frequent and desirable form of pay at the end of the $4^{\text {th }}$ century..$^{58}$ Thus, coins should not strictly be seen as evidence of administrative and economic activities in the province, but rather as an indicator of their level at the sites, and of the degree of monetization. ${ }^{59}$ This was a rural frontier military zone where the army was indirectly responsible for establishing any monetized local market that subsequently gravitated towards, and depended on, urban centres in the vicinity. ${ }^{60}$ Therefore, it is only reasonable to assume that the coin supply to the Teutoburgium and Cornacum area depended on military presence in terms of payments to regular garrisons ${ }^{61}$ It is then possible to draw a parallel between the cessation of the coin supply and the defensive significance of the forts and the limes itself in the province due to changed political, administrative, military, demographic, etc. conditions. However, it would be erroneously facile to connect it with the end of Roman authority over the territory (albeit that the latter probably followed the former). ${ }^{62}$

\section{Conclusion}

From the numismatic collection of the Archaeological Museum in Zagreb, a total of 618 Late Roman coins from the Valentinian dynasty onward, found in the area of Roman castella Teutoburgium (Dalj) and Cornacum (Sotin) have been examined, whilst the representative sample selected for further analysis consisted of 534 items. Among the coins identified, most numerous are $Æ 3$ nummi, and there are several $Æ 4$ and $Æ 2$ pieces, while cat. no. 249 and cat. no. 475 are Late Roman solidi, which have been omitted from the analysis. The data gathered has shown that coins from the Siscian mint are the best-represented at both localities, and its emissions are present throughout the majority of the period analysed. Other mints, such as Aquileia, Thessalonica and Roma are likewise significantly present. This data proved to be consistent with the wider limes area. Furthermore, the results showcase a parallel chronological development regarding coin circulation intensity until 378 and a concurrent end of the coin supply. According to the analysis, there is a possibility that the latest regular coin supply to Teutoburgium and Cornacum is related to a dispatch that occurred sometime between 388 and 393, although the size of the sample analysed is insufficient, and additional exploration of a wider area of the Croatian part of the limes is required for stating firm conclusions on the matter. Both localities demonstrate that the coins associated with the last recorded supply are $Æ 4$ SALVS REIPVBLICAE, minted for emperor Valentinian II (cat. nos 616-617) and Arcadius (cat. no. 182 and cat. no. 618) under Theodosius I's authority. The above results correlate with the data from the neighbouring frontier provinces.

To summarize, considering the present state of research, the coin supply to Teutoburgium and Cornacum and the fluctuation in coin circulation intensity might have been connected with the military and its activities at the Empire's frontier. Hence, the period between 388 and 393 could be a terminus post quem for the end of the regular Roman military presence in the studied part of the Danube limes, and therefore of the beginning of the disintegration of its structure, as well as of the decline in local administrative and economic activities as the result of the former. The aforesaid dating can be associated with the accounts of the loss of the Pannonian frontier provinces. Lastly, it must be underlined that further study is needed for providing more definite interpretations of numismatic material, while systematic archaeological excavations in the Croatian part of the Danube limes are highly desired.

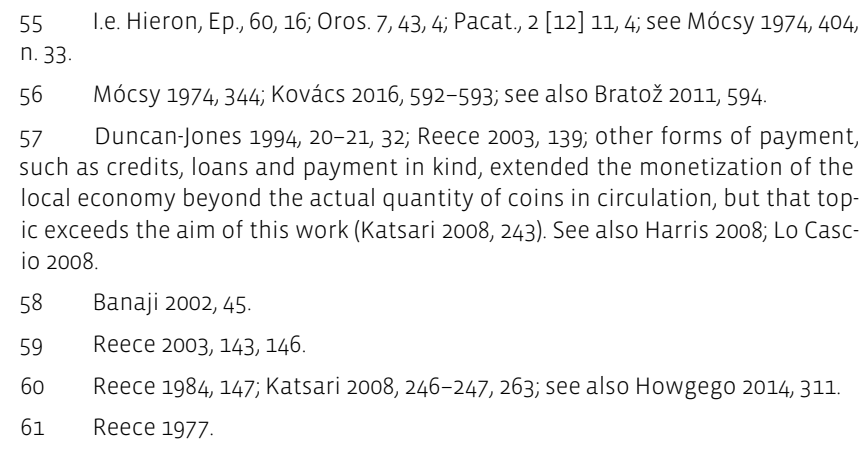

62 When thinking about the limes as a frontier system one should, as A. Mócsy has suggested, recognize the difference between the formal state frontier, frontiers of spheres of interest, and strategic lines occupied by the army (Mócsy 1974, 346). 


\section{Catalogue}

\begin{tabular}{|c|c|c|c|c|c|c|c|c|c|c|c|c|c|}
\hline \multicolumn{14}{|c|}{ DALJ } \\
\hline $\begin{array}{l}\text { CAT. } \\
\text { NO. }\end{array}$ & RULER & $\begin{array}{l}\text { DATING } \\
\text { (YRS) }\end{array}$ & DENOM. & MAT. & $\begin{array}{c}\text { D. } \\
(\mathrm{mm})\end{array}$ & $\begin{array}{l}\text { WT. } \\
\text { (g) }\end{array}$ & $\begin{array}{l}\text { ORI- } \\
\text { ENT. }\end{array}$ & OBVERSE & REVERSE & $\begin{array}{l}\text { MINT } \\
\text { MARKS }\end{array}$ & $\begin{array}{l}\text { REFEREN- } \\
\text { CE }\end{array}$ & $\begin{array}{l}\text { INV. } \\
\text { NO. }\end{array}$ & NOTE \\
\hline 1 & Valens & 364 & E 3 & E & 18 & 2.24 & 12 & $\begin{array}{l}\text { DN VALEN-S } \\
\text { PF AVG }\end{array}$ & $\begin{array}{c}\text { SECVRITAS } \\
\text { REIPVB[LICAE] }\end{array}$ & B SIRM & $\begin{array}{c}\text { RIC 159, } \\
7 \mathrm{~b}\end{array}$ & G133-44 & \\
\hline 2 & Valentinian I & 364 & $\star_{3} 3$ & 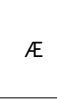 & 18 & 2.27 & 7 & $\begin{array}{c}\text { [DN V]ALEN- } \\
\text { TINI-ANVS PF } \\
\text { AVG }\end{array}$ & VOT IV|MVLT|X & B SIRM & RIC 159,8 & G133-116 & \\
\hline 3 & Valentinian I & $364-367$ & Æ3 & $E$ & 17 & 2.13 & 6 & $\begin{array}{c}{[D N]} \\
\text { VALENTIN[I- } \\
\text { A]NVS P[F } \\
\text { AVG] }\end{array}$ & $\begin{array}{c}\text { [GLORIA RO]- } \\
\text { MANORVM }\end{array}$ & $\begin{array}{c}--/ A / / S M \\
A Q P\end{array}$ & RIC 95,7a & G133-104 & \\
\hline 4 & Valentinian I & $364-367$ & E 3 & 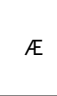 & 18 & 2.54 & 6 & $\begin{array}{l}\text { DN VALENTI- } \\
\text { NI-ANVS PF } \\
\text { AVG }\end{array}$ & $\begin{array}{l}\text { GLORIA RO-MA- } \\
\text { NORVM }\end{array}$ & A SIS C & $\begin{array}{c}\text { RIC 146, } \\
5 \mathrm{a}\end{array}$ & C31581 & \\
\hline 5 & Valentinian I & $364-367$ & $\notin_{3}$ & $\notin$ & 18 & 2.09 & 7 & $\begin{array}{c}\text { DN VALENTI- } \\
\text { NI-ANVS PF } \\
\text { AVG }\end{array}$ & $\begin{array}{c}\text { GLORIA RO-MA- } \\
\text { NORVM }\end{array}$ & $\cdot \Gamma \operatorname{SISC}$ & $\begin{array}{c}\text { RIC 146, } \\
5 a\end{array}$ & G133-6 & \\
\hline 6 & Valentinian I & $364-367$ & E 3 & $\notin$ & 18 & 2.26 & 11 & $\begin{array}{l}\text { DN VALENTI- } \\
\text { NI-ANVS PF } \\
\text { AVG }\end{array}$ & $\begin{array}{l}\text { GLORIA RO-MA- } \\
\text { NORVM }\end{array}$ & $D \Gamma S I S C$ & $\begin{array}{l}\text { RIC } 146, \\
5 a\end{array}$ & G126-9 & \\
\hline 7 & Valentinian I & $364-367$ & Æ 3 & $Æ$ & 18 & 2.65 & 6 & $\begin{array}{l}\text { DN VALENTI- } \\
\text { NI-ANVS PF } \\
\text { AVG }\end{array}$ & $\begin{array}{l}\text { GLORIA RO-MA- } \\
\text { NORVM }\end{array}$ & $\cdot \Gamma \operatorname{SISC}$ & $\begin{array}{c}\text { RIC 146, } \\
5 a\end{array}$ & G133-14 & \\
\hline 8 & Valentinian I & $364-367$ & Æ3 & $\notin$ & 18 & 2.78 & 5 & $\begin{array}{c}\text { DN VALENTI- } \\
\text { NI-ANVS PF } \\
\text { AVG }\end{array}$ & $\begin{array}{l}\text { GLORIA RO-MA- } \\
\text { NORVM }\end{array}$ & D ISISC & $\begin{array}{c}\text { RIC 146, } \\
5 a\end{array}$ & G133-26 & \\
\hline 9 & Valentinian I & $364-367$ & Æ3 & $\notin$ & 18 & 2.66 & 2 & $\begin{array}{c}\text { DN VALENTI- } \\
\text { NI-ANVS PF } \\
\text { AVG }\end{array}$ & $\begin{array}{l}\text { GLORIA RO-MA- } \\
\text { NORVM }\end{array}$ & A SIS C & $\begin{array}{c}\text { RIC 146, } \\
5 a\end{array}$ & G133-29 & \\
\hline 10 & Valentinian I & $364-367$ & $\notin 3$ & $\notin$ & 17 & 2.21 & 1 & $\begin{array}{l}\text { DN VALENTI- } \\
\text { NI-ANVS PF } \\
\text { AVG }\end{array}$ & $\begin{array}{l}\text { GLORIA RO-MA- } \\
\text { NORVM }\end{array}$ & $\mathrm{D} \Gamma[\mathrm{SISC} \mathrm{C}]$ & $\begin{array}{c}\text { RIC 146, } \\
5 a\end{array}$ & G133-90 & \\
\hline 11 & Valentinian I & $364-367$ & Æ3 3 & 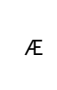 & 18 & 1.64 & 6 & $\begin{array}{c}\text { DN VALENTI- } \\
\text { NI-ANVS PF } \\
\text { AVG }\end{array}$ & $\begin{array}{l}\text { GLORIA RO]- } \\
\text { MANOR[VM] }\end{array}$ & TES B & $\begin{array}{c}\text { RIC } 176, \\
16 a\end{array}$ & G126-21 & \\
\hline 12 & Valentinian I & $364-367$ & $Æ_{3} 3$ & E & 19 & 1.91 & 12 & $\begin{array}{l}\text { DN VALENTI- } \\
\text { NI-ANVS PF } \\
\text { AVG }\end{array}$ & $\begin{array}{l}\text { GLORIA RO-MA- } \\
\text { NORVM }\end{array}$ & TES B & $\begin{array}{c}\text { RIC } 176, \\
16 a\end{array}$ & G133-71 & \\
\hline 13 & Valentinian I & $364-367$ & Æ3 & $\notin$ & 17 & 1.68 & 5 & $\begin{array}{c}\text { [DN] VALEN- } \\
\text { TINI-[ANVS } \\
\text { PF AVG] }\end{array}$ & $\begin{array}{c}\text { SECVRITAS } \\
\text { REIPVBLICAE }\end{array}$ & $\begin{array}{l}\text { OF/III// } \\
\text { CONST }\end{array}$ & RIC 64, 9a & G133-32 & \\
\hline 14 & Valentinian I & $364-367$ & E 3 & $\notin$ & 18 & 2.38 & 6 & $\begin{array}{l}\text { DN VALENTI- } \\
\text { NI-ANVS PF } \\
\text { AVG }\end{array}$ & $\begin{array}{c}\text { SECVRITAS } \\
\text { REIPVBLICAE }\end{array}$ & $\triangle S I S C$ & $\begin{array}{c}\text { RIC 146, } \\
7 a\end{array}$ & С31584 & \\
\hline 15 & Valentinian I & $364-367$ & $Æ_{3} 3$ & $\notin$ & 19 & 2.28 & 1 & $\begin{array}{l}\text { DN VALENTI- } \\
\text { NI-ANVS PF } \\
\text { AVG }\end{array}$ & $\begin{array}{l}\text { SECVRITAS } \\
\text { REIPVBLICAE }\end{array}$ & $\cdot \Delta$ SIS C & $\begin{array}{c}\text { RIC 146, } \\
7 a\end{array}$ & G126-5 & \\
\hline 16 & Valentinian I & $364-367$ & E 3 & $\notin$ & 18 & 2.53 & 12 & $\begin{array}{c}\text { DN VALENTI- } \\
\text { NI-ANVS PF } \\
\text { AVG }\end{array}$ & $\begin{array}{l}\text { SECVRITAS } \\
\text { REIPVBLICAE }\end{array}$ & $\begin{array}{c}* \mathrm{~A} /--/ / \mathrm{D} \Delta \\
\mathrm{SISC}\end{array}$ & $\begin{array}{c}\text { RIC } 146, \\
7 a\end{array}$ & G133-5 & \\
\hline 17 & Valentinian I & $364-367$ & $\star_{3} 3$ & $\notin$ & 19 & 2.72 & 12 & $\begin{array}{c}\text { [DN } \\
\text { VALENTINI]- } \\
\text { ANV[S PF } \\
\text { AVG] }\end{array}$ & $\begin{array}{l}\text { [SECVRITAS] } \\
\text { REIP[VBLICAE] }\end{array}$ & B SIS C & $\begin{array}{c}\text { RIC 146, } \\
7 a\end{array}$ & G133-25 & \\
\hline 18 & Valentinian I & $364-367$ & Æ3 & $\notin$ & 18 & 1.94 & 7 & $\begin{array}{l}\text { DN VALENTI- } \\
\text { NI-ANVS PF } \\
\text { AVG }\end{array}$ & $\begin{array}{c}\text { SECVRITAS } \\
\text { REIPVBLICAE }\end{array}$ & "「SIS C & $\begin{array}{c}\text { RIC 146, } \\
5 a\end{array}$ & G133-65 & \\
\hline 19 & Valentinian I & $364-367$ & E 3 & $\notin$ & 20 & 2.16 & 12 & $\begin{array}{l}\text { DN VALENTI- } \\
\text { NI-ANVS PF } \\
\text { AVG }\end{array}$ & $\begin{array}{l}\text { SECVRITAS } \\
\text { REIPVBLICAE }\end{array}$ & D ISIS C & $\begin{array}{c}\text { RIC 146, } \\
5 a\end{array}$ & G133-66 & \\
\hline 20 & Valentinian I & $364-367$ & Æ 3 & $\notin$ & 18 & 2.34 & 12 & $\begin{array}{l}\text { DN VALENTI- } \\
\text { NI-[ANVS PF } \\
\text { AVG ] }\end{array}$ & $\begin{array}{c}\text { [SECVRITAS] } \\
\text { REIP[VBLICAE] }\end{array}$ & $\begin{array}{c}* \mathrm{~A} /--/ / ? \\
\Delta \mathrm{SISC}\end{array}$ & $\begin{array}{c}\text { RIC 146, } \\
7 a\end{array}$ & G133-87 & \\
\hline
\end{tabular}




\begin{tabular}{|c|c|c|c|c|c|c|c|c|c|c|c|c|c|}
\hline \multicolumn{14}{|c|}{ DALJ } \\
\hline $\begin{array}{l}\text { CAT. } \\
\text { NO. }\end{array}$ & RULER & $\begin{array}{l}\text { DATING } \\
\text { (YRS) }\end{array}$ & DENOM. & MAT. & $\begin{array}{c}\mathrm{D} . \\
(\mathrm{mm})\end{array}$ & $\begin{array}{l}\text { WT. } \\
\text { (g) }\end{array}$ & $\begin{array}{l}\text { ORI- } \\
\text { ENT. }\end{array}$ & OBVERSE & REVERSE & $\begin{array}{l}\text { MINT } \\
\text { MARKS }\end{array}$ & $\begin{array}{l}\text { REFE- } \\
\text { RENCE }\end{array}$ & $\begin{array}{l}\text { INV. } \\
\text { NO. }\end{array}$ & NOTE \\
\hline 21 & Valentinian I & $364-367$ & Æ 3 & $\mathscr{F}$ & 18 & 2.04 & 1 & $\begin{array}{l}\text { DN VALENTI- } \\
\text { NI-ANVS PF } \\
\text { AVG }\end{array}$ & $\begin{array}{l}\text { SECVRITAS } \\
\text { REIPVBLICAE }\end{array}$ & $\begin{array}{c}* \mathrm{~A} /--/ / \mathrm{DA} \\
\mathrm{SISC}\end{array}$ & $\begin{array}{c}\text { RIC } 146 \\
7 a\end{array}$ & G133-89 & \\
\hline 22 & Valentinian I & $364-367$ & $Æ_{3} 3$ & $F$ & 18 & 3 & 7 & $\begin{array}{c}\text { DN VALENTI- } \\
\text { NI-ANVS PF } \\
\text { AVG }\end{array}$ & $\begin{array}{l}\text { SECVRITAS } \\
\text { REIPVBLICAE }\end{array}$ & A SIS C & $\begin{array}{l}\text { RIC } 146 \\
7 a\end{array}$ & G133-93 & \\
\hline 23 & Valentinian I & $364-367$ & Æ 3 & $\mathscr{F}$ & 18 & 1.12 & 6 & $\begin{array}{c}\text { [DN VALENT] } \\
\text { INI-ANVS PF } \\
\text { A[VG] }\end{array}$ & $\begin{array}{l}\text { SE[CVRITAS } \\
\text { REIPVBLI]CAE }\end{array}$ & ?/--//TESA & $\begin{array}{c}\text { RIC 176, } \\
18 \mathrm{a}\end{array}$ & G126-19 & \\
\hline 24 & Valentinian I & $364-367$ & $Æ_{3}$ & $\mathscr{E}$ & 18 & 1.62 & 12 & $\begin{array}{c}\text { DN VALENTI- } \\
\text { NI-ANVS PF } \\
\text { AVG }\end{array}$ & $\begin{array}{l}\text { SECVRITAS } \\
\text { REIPVBLICAE }\end{array}$ & TES A & $\begin{array}{l}\text { RIC 176, } \\
18 \mathrm{a}\end{array}$ & G137-3 & \\
\hline 25 & Valentinian I & $364-367$ & $\mathbb{E}_{3}$ & $\mathscr{E}$ & 19 & 3.1 & 12 & $\begin{array}{l}\text { DN VALENTI- } \\
\text { NI-ANVS PF } \\
\text { AVG }\end{array}$ & $\begin{array}{l}\text { RESTITV-TOR } \\
\text { REIP }\end{array}$ & A SIS C & $\begin{array}{c}\text { RIC 146, } \\
6 \mathrm{a}\end{array}$ & G137-2 & \\
\hline 26 & Valentinian I & $364-367$ & Æ 3 & $\mathscr{F}$ & 18 & 2.61 & 5 & $\begin{array}{c}\text { DN } \\
\text { VALENT[INI]- } \\
\text { ANVS PF AVG }\end{array}$ & $\begin{array}{l}\text { RESTITV-TOR } \\
\text { REIP }\end{array}$ & TES B & $\begin{array}{c}\text { RIC 176 } \\
17 a\end{array}$ & G126-17 & \\
\hline 27 & $\begin{array}{l}\text { Valent. I/ } \\
\text { Valens }\end{array}$ & $364-367$ & $Æ 3$ & E & 19 & 1.48 & 11 & DN VALEN ? & $\begin{array}{l}\text { RES[TITV-TOR } \\
\text { REIP] }\end{array}$ & P CONST & $\begin{array}{l}\text { RIC 64, } \\
8 \mathrm{a}-\mathrm{c}\end{array}$ & G133-120 & \\
\hline 28 & $\begin{array}{l}\text { Valent. I/ } \\
\text { Valens }\end{array}$ & $364-367$ & Æ 3 & E & 18 & 1.91 & 5 & $?$ & $\begin{array}{l}\text { SECVRITAS } \\
\text { REIPVBLICAE }\end{array}$ & $\begin{array}{l}\mathrm{OF} / \mathrm{I} \cdot / / \\
\mathrm{CO}[\mathrm{N}]\end{array}$ & $\begin{array}{l}\text { RIC 64, } \\
9 a / b\end{array}$ & G133-82 & \\
\hline 29 & Valens & $364-367$ & $\mathbb{E}_{3}$ & E & 19 & 1.78 & 7 & $\begin{array}{l}\text { DN VALEN-[S] } \\
\text { PF AV[G] }\end{array}$ & $\begin{array}{l}\text { GLORIA [RO- } \\
\text { MAN]ORVM }\end{array}$ & $\begin{array}{c}--/ \text { cres- } \\
\text { cent//SM } \\
\text { AQS S }\end{array}$ & RIC $95,7 b$ & G126-13 & \\
\hline 30 & Valens & $364-367$ & Æ 3 & E & 18 & 2.11 & 6 & $\begin{array}{l}\text { DN VALEN-S } \\
\text { PF AVG }\end{array}$ & $\begin{array}{l}\text { GLORIA RO-MA- } \\
\text { NORVM }\end{array}$ & $\begin{array}{c}\text { ?--/B//SM } \\
\text { AQP }\end{array}$ & RIC $95,9 b$ & G133-61 & \\
\hline 31 & Valens & $364-367$ & $\mathbb{E}_{3} 3$ & E & 18 & 2.75 & 12 & $\begin{array}{l}\text { DN V[ALEN]-S } \\
\text { PF AVG }\end{array}$ & $\begin{array}{l}\text { GLORIA RO- } \\
\text { [MANO]RVM }\end{array}$ & $\begin{array}{l}\text { crescent } \\
\text { SM AQS }\end{array}$ & RIC 95, 7b & G133-95 & \\
\hline 32 & Valens & $364-367$ & $\notin_{3}$ & E & 18 & 2.3 & 6 & $\begin{array}{l}\text { DN VALEN-S } \\
\text { [PF AVG] }\end{array}$ & $\begin{array}{l}\text { [GLORIA RO- } \\
\text { MANORVM] }\end{array}$ & $\begin{array}{c}--/ A / / S[M] \\
A Q S\end{array}$ & RIC $95,7 b$ & G133-109 & \\
\hline 33 & Valens & $364-367$ & $\notin 3$ & E & 18 & 2.2 & 6 & $\begin{array}{l}\text { DN VALEN-S } \\
\text { PF AVG }\end{array}$ & $\begin{array}{l}\text { [GLORIA RO]- } \\
\text { MANORVM }\end{array}$ & - B SIS C & $\begin{array}{c}\text { RIC } 146 \\
5 b\end{array}$ & G126-10 & \\
\hline 34 & Valens & $364-367$ & $Æ_{3} 3$ & E & 18 & 2.4 & 12 & $\begin{array}{l}\text { DN VALEN-S } \\
\text { PF AVG }\end{array}$ & $\begin{array}{l}\text { GLORIA RO- } \\
\text { [MANORVM] }\end{array}$ & "B SIS C & $\begin{array}{l}\text { RIC 146, } \\
5 \mathrm{~b}\end{array}$ & G133-16 & \\
\hline 35 & Valens & $364-367$ & E 3 & E & 18 & 2.03 & 2 & $\begin{array}{l}\text { DN [VALEN]-S } \\
\text { PF AVG }\end{array}$ & $\begin{array}{c}\text { GLORIA RO-MA- } \\
\text { NORVM }\end{array}$ & B SIS C & $\begin{array}{l}\text { RIC 146, } \\
5 \mathrm{~b}\end{array}$ & G133-21 & \\
\hline 36 & Valens & $364-367$ & E 3 & E & 18 & 2.29 & 7 & $\begin{array}{c}\text { DN [VAL]EN-S } \\
\text { PF AVG }\end{array}$ & $\begin{array}{l}\text { GLORIA RO-MA- } \\
\text { NORVM }\end{array}$ & $\begin{array}{c}--/ * \mathrm{~A} / / \mathrm{DB} \\
\mathrm{SISC}\end{array}$ & $\begin{array}{c}\text { RIC } 146 \\
5 \mathrm{~b}\end{array}$ & G133-23 & \\
\hline 37 & Valens & $364-367$ & $\notin_{3} 3$ & E & 19 & 2.59 & 12 & $\begin{array}{l}\text { DN VALEN-S } \\
\text { PF AVG }\end{array}$ & $\begin{array}{l}\text { GLORIA RO- } \\
\text { [MANORVM] }\end{array}$ & - B SIS C & $\begin{array}{l}\text { RIC } 146 \text {, } \\
5 \mathrm{~b}\end{array}$ & G133-46 & \\
\hline 38 & Valens & $364-367$ & E 3 & E & 18 & 2.62 & 6 & $\begin{array}{l}\text { DN VALEN-S } \\
\text { PF AVG }\end{array}$ & $\begin{array}{l}\text { [GLORIA RO]- } \\
\text { MANORVM }\end{array}$ & $\begin{array}{c}--/ * \mathrm{~A} / / \cdot \mathrm{B} \\
\mathrm{SISC}\end{array}$ & $\begin{array}{l}\text { RIC 146, } \\
5 \mathrm{~b}\end{array}$ & G133-56 & \\
\hline 39 & Valens & $364-367$ & Æ 3 & E & 18 & 2.7 & 6 & $\begin{array}{l}\text { DN VALEN-S } \\
\text { PF AVG }\end{array}$ & $\begin{array}{c}\text { GLORIA RO-MA- } \\
\text { NORVM }\end{array}$ & $\begin{array}{c}--/ * \mathrm{~A} / / \mathrm{D} \mathrm{B} \\
\mathrm{SISC}\end{array}$ & $\begin{array}{l}\text { RIC 146, } \\
5 \mathrm{~b}\end{array}$ & G133-73 & \\
\hline 40 & Valens & $364-367$ & E 3 & E & 18 & 2.13 & 2 & $\begin{array}{l}\text { DN VALEN-S } \\
\text { PF AVG }\end{array}$ & $\begin{array}{c}\text { GLORIA RO-MA- } \\
\text { NORVM }\end{array}$ & D B SIS C & $\begin{array}{l}\text { RIC 146, } \\
5 \mathrm{~b}\end{array}$ & G133-83 & \\
\hline 41 & Valens & $364-367$ & E 3 & E & 18 & 1.91 & 1 & $\begin{array}{l}\text { DN VALEN-S } \\
\text { PF AVG }\end{array}$ & $\begin{array}{l}\text { GLORIA RO-MA- } \\
\text { NORVM }\end{array}$ & B SIS C & $\begin{array}{l}\text { RIC 146, } \\
5 \mathrm{~b}\end{array}$ & G133-86 & \\
\hline 42 & Valens & $364-367$ & $\mathbb{E}_{3} 3$ & E & 18 & 2.37 & 7 & $\begin{array}{l}\text { DN VALE[N-S } \\
\text { P]F AVG }\end{array}$ & $\begin{array}{l}\text { [GLORIA RO- } \\
\text { MANO]RVM }\end{array}$ & $\begin{array}{l}--/ ? D B \\
\text { SIS C }\end{array}$ & $\begin{array}{l}\text { RIC 146, } \\
5 \mathrm{~b}\end{array}$ & G133-97 & \\
\hline 43 & Valens & $364-367$ & $Æ_{3} 3$ & E & 19 & 1.09 & 1 & $\begin{array}{c}\text { [DN VALEN-S } \\
\text { PF] AVG }\end{array}$ & $\begin{array}{l}\text { GL[ORIA RO- } \\
\text { MANO]RVM }\end{array}$ & DA SIS C & $\begin{array}{l}\text { RIC 146, } \\
5 \mathrm{~b}\end{array}$ & G133-105 & \\
\hline 44 & Valens & $364-367$ & Æ 3 & EE & 17 & 2.1 & 12 & $\begin{array}{c}\text { DN [VAL]EN-S } \\
\text { PF AVG }\end{array}$ & $\begin{array}{c}\text { GLORIA RO-MA- } \\
\text { NORVM }\end{array}$ & TES $\Gamma$ & $\begin{array}{l}\text { RIC 176, } \\
16 \mathrm{~b}\end{array}$ & G133-24 & \\
\hline 45 & Valens & $364-367$ & $E_{3}$ & E & 18 & 2.06 & 11 & $\begin{array}{l}\text { DN VALEN-S } \\
\text { PF AVG }\end{array}$ & $\begin{array}{l}\text { [GLOR]IA RO- } \\
\text { MANORVM }\end{array}$ & TES $\Gamma$ & $\begin{array}{l}\text { RIC } 176 \\
16 \mathrm{~b}\end{array}$ & G133-103 & \\
\hline 46 & Valens & $364-367$ & $\notin_{3}$ & EE & 17 & 1.97 & 7 & $\begin{array}{l}\text { DN VALEN-S } \\
\text { [PF AVG] }\end{array}$ & $\begin{array}{l}\text { GLORIA RO- } \\
\text { [MANORVM] }\end{array}$ & CONS $\Gamma$ & $\begin{array}{l}\text { RIC 214 } \\
16 \mathrm{~b}\end{array}$ & G133-126 & \\
\hline 47 & Valens & $364-367$ & Æ 3 & E & 18 & 1.45 & 5 & $\begin{array}{l}\text { DN V[AL]EN-S } \\
\text { PF AVG }\end{array}$ & $\begin{array}{l}\text { [SECVR]ITAS } \\
\text { REIPVBLICAE }\end{array}$ & $\begin{array}{l}\text { OF/III// } \\
\text { CONST }\end{array}$ & RIC $64,9 \mathrm{~b}$ & G135-3 & \\
\hline 48 & Valens & $364-367$ & E 3 & $\mathscr{E}$ & 18 & 1.93 & 6 & $\begin{array}{l}\text { DN VALEN-S } \\
\text { PF AVG }\end{array}$ & $\begin{array}{c}\text { SECVR[ITAS REI] } \\
\text { PVBLICAE }\end{array}$ & $* \mathrm{SM} A Q \mathrm{P}$ & RIC $95,9 b$ & G134-2 & \\
\hline 49 & Valens & $364-367$ & Æ 3 & E & 17 & 1.67 & 5 & $\begin{array}{c}\text { DN VALEN-[S] } \\
\text { PF [AVG] }\end{array}$ & $\begin{array}{c}\text { SECVRIT[AS] } \\
\text { REI[PVBLICAE] }\end{array}$ & $R B$ & $\begin{array}{l}\text { RIC 120, } \\
17 \mathrm{~b}\end{array}$ & G126-18 & \\
\hline
\end{tabular}




\begin{tabular}{|c|c|c|c|c|c|c|c|c|c|c|c|c|c|}
\hline \multicolumn{14}{|c|}{ DALJ } \\
\hline $\begin{array}{l}\text { CAT. } \\
\text { NO. }\end{array}$ & RULER & $\begin{array}{c}\text { DATING } \\
\text { (YRS) }\end{array}$ & DENOM. & MAT. & $\begin{array}{c}\mathrm{D} . \\
(\mathrm{mm})\end{array}$ & $\begin{array}{l}\text { WT. } \\
\text { (g) }\end{array}$ & $\begin{array}{l}\text { ORI- } \\
\text { ENT. }\end{array}$ & OBVERSE & REVERSE & $\begin{array}{c}\text { MINT } \\
\text { MARKS }\end{array}$ & $\begin{array}{l}\text { REFE- } \\
\text { RENCE }\end{array}$ & $\begin{array}{l}\text { INV. } \\
\text { NO. }\end{array}$ & NOTE \\
\hline 50 & Valens & $364-367$ & Æ 3 & $A E$ & 19 & 2.57 & 7 & $\begin{array}{c}\text { DN VALEN-S } \\
\text { PF AVG }\end{array}$ & $\begin{array}{c}\text { SECVRITAS } \\
\text { REIPVBLICAE }\end{array}$ & B SIS C & $\begin{array}{c}\text { RIC } 146, \\
7 b\end{array}$ & С 31588 & \\
\hline 51 & Valens & $364-367$ & $\notin 3$ & E & 18 & 2.41 & 1 & $\begin{array}{c}\text { DN VALEN-S } \\
\text { PF AVG }\end{array}$ & $\begin{array}{c}\text { SEC[VRITAS } \\
\text { REIPV]BLICAE }\end{array}$ & ? A SIS C & RIC $146,7 b$ & G126-16 & \\
\hline 52 & Valens & $364-367$ & $Æ_{3}$ & $E$ & 19 & 2.79 & 5 & $\begin{array}{c}\text { [DN VALEN]-S } \\
{[P F] \text { AVG }}\end{array}$ & $\begin{array}{c}\text { [SECVRI]TAS } \\
\text { [REIPVBLICAE] }\end{array}$ & $\mathrm{DA}$ [SIS C] & RIC $146,7 b$ & G126-22 & \\
\hline 53 & Valens & $364-367$ & Æ 3 & EE & 18 & 2.07 & 6 & $\begin{array}{l}\text { DN VALEN-S } \\
\text { PF AVG }\end{array}$ & $\begin{array}{c}\text { SECVRITAS } \\
\text { REIPVBLICAE }\end{array}$ & - A SIS C & $\begin{array}{c}\text { RIC } 146, \\
7 b\end{array}$ & G133-2 & \\
\hline 54 & Valens & $364-367$ & $Æ 3$ & $\mathscr{E}$ & 18 & 1.96 & 11 & $\begin{array}{c}\text { DN VALEN-S } \\
\text { PF AVG }\end{array}$ & $\begin{array}{c}\text { SECVRITAS } \\
\text { REIPVBLICAE }\end{array}$ & $\begin{array}{l}\text { BA/--//D A } \\
\text { SIS C }\end{array}$ & $\begin{array}{c}\text { RIC } 146, \\
7 b\end{array}$ & G133-4 & \\
\hline 55 & Valens & $364-367$ & $Æ_{3}$ & AE & 18 & 2.17 & 6 & $\begin{array}{l}\text { DN VALEN-S } \\
\text { PF AVG }\end{array}$ & $\begin{array}{l}\text { SECVRITAS } \\
\text { REIPVBLICAE }\end{array}$ & - A SIS C & $\begin{array}{c}\text { RIC } 146, \\
7 b\end{array}$ & G133-17 & \\
\hline 56 & Valens & $364-367$ & $\notin 3$ & $\mathscr{A E}$ & 19 & 2.11 & 12 & $\begin{array}{c}\text { DN VALEN-S } \\
\text { PF AVG }\end{array}$ & $\begin{array}{l}\text { SECVRITAS } \\
\text { REIPVBLICAE }\end{array}$ & - B SIS C & $\begin{array}{c}\text { RIC 146, } \\
5 b\end{array}$ & G133-35 & \\
\hline 57 & Valens & $364-367$ & $Æ_{3}$ & AE & 18 & 2.16 & 7 & $\begin{array}{l}\text { DN VALEN-S } \\
\text { PF AVG }\end{array}$ & $\begin{array}{c}\text { SECVRITAS } \\
\text { REIPVBLICAE }\end{array}$ & B SIS C & $\begin{array}{c}\text { RIC } 146 \\
7 b\end{array}$ & G133-48 & \\
\hline 58 & Valens & $364-367$ & $Æ 3$ & $\mathscr{A E}$ & 20 & 2.24 & 7 & $\begin{array}{c}\text { DN VALEN-S } \\
\text { PF AVG }\end{array}$ & $\begin{array}{c}\text { SECVRITAS } \\
\text { REIPVBLICAE }\end{array}$ & $\cdot \Delta \mathrm{SISC}$ & $\begin{array}{c}\text { RIC } 146, \\
7 b\end{array}$ & G133-49 & \\
\hline 59 & Valens & $364-367$ & $\mathbb{E}_{3}$ & FE & 18 & 2.31 & 7 & $\begin{array}{l}\text { DN VALEN-S } \\
\text { PF AVG }\end{array}$ & $\begin{array}{c}\text { SECVRITAS } \\
\text { [REIPVBLICAE] }\end{array}$ & A SI[SC] & $\begin{array}{c}\text { RIC } 146, \\
7 b\end{array}$ & G133-59 & \\
\hline 60 & Valens & $364-367$ & $Æ 3$ & E & 17 & 2.19 & 7 & $\begin{array}{c}\text { DN VALEN-S } \\
\text { PF AVG }\end{array}$ & $\begin{array}{l}\text { SECVRITAS } \\
\text { REIPVBLICAE }\end{array}$ & $\Delta \mathrm{SISC}$ & $\begin{array}{c}\text { RIC } 146 \\
7 a\end{array}$ & G133-63 & \\
\hline 61 & Valens & $364-367$ & $\mathbb{E}_{3}$ & $\mathscr{A E}$ & 18 & 2.34 & 11 & $\begin{array}{c}\text { DN VALEN-S } \\
{[\text { PF AVG] }}\end{array}$ & $\begin{array}{l}\text { SECVRITAS } \\
\text { REIPVBLICAE }\end{array}$ & D A SIS C & $\begin{array}{c}\text { RIC } 146, \\
7 b\end{array}$ & G133-75 & \\
\hline 62 & Valens & $364-367$ & E 3 & E & 18 & 2.41 & 5 & $\begin{array}{c}\text { DN VALEN-S } \\
\text { PF AVG }\end{array}$ & $\begin{array}{l}\text { SECVRITAS } \\
\text { REIPVBLICAE }\end{array}$ & $\begin{array}{l}\text { DA/--//D A } \\
\text { SIS C }\end{array}$ & $\begin{array}{c}\text { RIC } 146, \\
7 b\end{array}$ & G133-76 & \\
\hline 63 & Valens & $364-367$ & E3 & 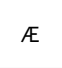 & 19 & 1.79 & 1 & $\begin{array}{l}\text { DN VALEN-S } \\
\text { PF AVG }\end{array}$ & $\begin{array}{c}\text { SECVRITAS } \\
\text { REIPVBLICAE }\end{array}$ & - A SIS C & $\begin{array}{c}\text { RIC } 146, \\
7 b\end{array}$ & G133-78 & \\
\hline 64 & Valens & $364-367$ & E3 3 & E & 17 & 1.89 & 7 & $\begin{array}{c}\text { DN VALEN-S } \\
\text { PF AVG }\end{array}$ & $\begin{array}{l}\text { SECVRITAS } \\
\text { REIPVBLICAE }\end{array}$ & $\begin{array}{l}\text { AA/--//D A } \\
\text { SIS C }\end{array}$ & $\begin{array}{c}\text { RIC } 146, \\
7 b\end{array}$ & G133-84 & \\
\hline 65 & Valens & $364-367$ & $E_{3}$ & $\notin E$ & 18 & 2.56 & 7 & $\begin{array}{c}\text { DN VALEN-[S } \\
\text { PF AVG] }\end{array}$ & $\begin{array}{l}\text { SECVRITAS } \\
\text { REIPVBLICAE }\end{array}$ & D A SIS C & RIC $146,7 b$ & G133-107 & \\
\hline 66 & Valens & $364-367$ & $E_{3}$ & $A$ & 19 & 2.15 & 11 & $\begin{array}{c}\text { DN VALEN-S } \\
\text { PF AVG }\end{array}$ & $\begin{array}{l}\text { SECVRITAS } \\
\text { REIPVBLICAE }\end{array}$ & TES $\Delta$ & $\begin{array}{c}\text { RIC } 176 \\
18 \mathrm{~b}\end{array}$ & G133-113 & \\
\hline 67 & Valens & $364-367$ & E 3 & EE & 18 & 1.91 & 11 & $\begin{array}{c}\text { DN VALEN-S } \\
\text { PF AVG }\end{array}$ & $\begin{array}{c}\text { SECVRITAS } \\
\text { REIPVB[LICAE] }\end{array}$ & ?/--//TES A & $\begin{array}{c}\text { RIC } 176 \\
18 \mathrm{~b}\end{array}$ & G133-121 & \\
\hline 68 & Valens & $364-367$ & $E_{3}$ & EE & 20 & 2.49 & 5 & $\begin{array}{c}\text { DN VALEN- [S } \\
\text { PF AVG] }\end{array}$ & $\begin{array}{l}\text { RESTITV-[TOR] } \\
\text { REIP }\end{array}$ & B SIS C & $\begin{array}{c}\text { RIC } 146, \\
6 b\end{array}$ & C31586 & \\
\hline 69 & Valentinian I & $364-375$ & E 3 & $A E$ & 18 & 2.09 & 11 & $\begin{array}{c}\text { [DN V]ALEN- } \\
\text { TINI-ANVS PF } \\
\text { AVG }\end{array}$ & $\begin{array}{l}\text { [GLOR]IA RO- } \\
\text { [MANORVM] }\end{array}$ & $?$ & incerta & G133-101 & \\
\hline 70 & Valentinian I & $364-375$ & $E_{3}$ & E & 18 & 2.67 & 11 & $\begin{array}{c}\text { [DN VALE] } \\
\text { NTINI-ANVS } \\
\text { PF AVG] }\end{array}$ & $\begin{array}{l}\text { [GLORIA RO- } \\
\text { MAN[ORVM] }\end{array}$ & $?$ & incerta & G133-115 & \\
\hline 71 & Valentinian I & $364-375$ & $E_{3}$ & EE & 18 & 1.6 & 12 & $\begin{array}{c}\text { [DN V]ALEN- } \\
\text { TINI-ANVS PF } \\
\text { AVG }\end{array}$ & $\begin{array}{c}\text { [GL]OR[IA] RO- } \\
\text { MANORVM }\end{array}$ & $?$ & incerta & G133-118 & \\
\hline 72 & Valentinian I & $364-367$ & $E_{3}$ & E & 18 & 1.5 & 11 & $\begin{array}{c}\text { DN VALENTI- } \\
\text { NI-ANVS PF } \\
\text { AVG }\end{array}$ & $\begin{array}{l}\text { [SECVRITAS] } \\
\text { REIPVBLICAE }\end{array}$ & $\begin{array}{l}\text { OF/I// } \\
\text { CONST }\end{array}$ & RIC 64, 9a & G135-2 & \\
\hline 73 & Valentinian I & $364-375$ & $E_{3}$ & E & 18 & 1.52 & 6 & $\begin{array}{c}\text { [DN VALENTI- } \\
\text { NI-ANV]S PF } \\
\text { AVG }\end{array}$ & $\begin{array}{l}\text { [SECVRITAS } \\
\text { REIPVBLICAE] }\end{array}$ & ? SMAQS & $\begin{array}{c}\text { RIC } 95 / 96 \\
7 a / 11 a\end{array}$ & G133-127 & \\
\hline 74 & Valentinian I & $364-375$ & $E_{3}$ & E & 17 & 2.31 & 12 & $\begin{array}{c}\text { DN VALENTI- } \\
\text { NI-ANVS PF } \\
\text { AVG }\end{array}$ & $\begin{array}{c}\text { [SECVRITAS] } \\
\text { [REIPVBLICAE] }\end{array}$ & ? SIS C & incerta & G133-22 & \\
\hline 75 & Valentinian I & $364-375$ & $E_{3}$ & EE & 17 & 2.06 & 11 & $\begin{array}{c}\text { [DN } \\
\text { VALENTINI]- } \\
\text { ANVS PF } \\
{[A V G]}\end{array}$ & $\begin{array}{c}\text { [SE]CVRITAS } \\
\text { R[EIPVBLICAE] }\end{array}$ & $?$ & incerta & G133-128 & \\
\hline 76 & Valens & $364-375$ & $E_{3}$ & EE & 15 & 1.7 & 6 & $\begin{array}{c}\text { [DN ] VALEN- } \\
\text { S P[F AVG ] }\end{array}$ & $\begin{array}{c}\text { SECVRITAS } \\
\text { REIPVBLICAE }\end{array}$ & SM K A & $\begin{array}{l}\text { RIC 241, } \\
11 \mathrm{~b} / 13 \mathrm{~b}\end{array}$ & G133-94 & $\begin{array}{l}\text { issues } \\
\text { indi- } \\
\text { stingu- } \\
\text { ishable }\end{array}$ \\
\hline
\end{tabular}




\begin{tabular}{|c|c|c|c|c|c|c|c|c|c|c|c|c|c|}
\hline \multicolumn{14}{|c|}{ DALJ } \\
\hline $\begin{array}{l}\text { CAT. } \\
\text { NO. }\end{array}$ & RULER & $\begin{array}{c}\text { DATING } \\
\text { (YRS) }\end{array}$ & DENOM. & MAT. & $\begin{array}{c}\text { D. } \\
(\mathrm{mm})\end{array}$ & $\begin{array}{l}\text { WT. } \\
\text { (g) }\end{array}$ & $\begin{array}{l}\text { ORI- } \\
\text { ENT. }\end{array}$ & OBVERSE & REVERSE & $\begin{array}{l}\text { MINT } \\
\text { MARKS }\end{array}$ & $\begin{array}{l}\text { REFE- } \\
\text { RENCE }\end{array}$ & $\begin{array}{l}\text { INV. } \\
\text { NO. }\end{array}$ & NOTE \\
\hline 77 & Valens & $364-378$ & E 3 & $\notin$ & 18 & 1.59 & 6 & $\begin{array}{c}\text { [DN VALE]N-S } \\
\text { PF AVG }\end{array}$ & $\begin{array}{c}\text { [SECVRITAS } \\
\text { REIPVBLICAE] }\end{array}$ & $?$ & incerta & G133-102 & \\
\hline 78 & Valens & $364-378$ & Æ 3 & $\notin$ & 18 & 2.44 & 5 & $\begin{array}{l}\text { DN VALEN-S } \\
\text { PF AVG }\end{array}$ & $\begin{array}{c}\text { SECV[RITAS] } \\
\text { R[EIPVBLICAE] }\end{array}$ & $?$ & incerta & G133-114 & \\
\hline 79 & Valens & $364-378$ & Æ 3 & $\notin$ & 18 & 1.46 & 1 & $\begin{array}{c}\text { [DN V]ALEN-S } \\
\text { PF AVG }\end{array}$ & $\begin{array}{l}\text { [SE]CVRITAS } \\
\text { REIPVBL[ICAE] }\end{array}$ & $?$ & incerta & G133-117 & \\
\hline 80 & Valens & $364-378$ & $\mathbb{E}_{3}$ & $E$ & 16 & 3.11 & 6 & $\begin{array}{l}\text { DN VAL[EN]-S } \\
\text { PF AVG }\end{array}$ & $\begin{array}{l}\text { [SECVRITAS] } \\
\text { REIPVBL[ICAE] }\end{array}$ & $?$ & incerta & G133-124 & \\
\hline 81 & Valens & $364-378$ & $A_{3}$ & $\mathbb{E}$ & 17 & 2.45 & 5 & $\begin{array}{c}\text { [DN VA]LEN-S } \\
\text { PF AVG] }\end{array}$ & $\begin{array}{c}\text { *SEC]VRITAS } \\
\text { [REIPVBLICAE] }\end{array}$ & $?$ & incerta & G133-131 & \\
\hline 82 & Valens & $364-378$ & $A_{3} 3$ & $\mathbb{E}$ & 16 & 1.47 & 5 & $\begin{array}{l}\text { DN VALEN-[S } \\
\text { PF AVG] }\end{array}$ & $\begin{array}{l}\text { [SECVRIT]AS } \\
\text { [REI]PVBLICAE }\end{array}$ & $?$ & incerta & G133-132 & \\
\hline 83 & Valens & $364-378$ & Æ3 & $\mathbb{E}$ & 17 & 2.86 & 12 & $\begin{array}{c}\text { [DN VALEN]-S } \\
\text { PF AVG }\end{array}$ & $\begin{array}{l}\text { [SECVRITAS] } \\
\text { REIPVBLICAE }\end{array}$ & ? & incerta & G133-133 & \\
\hline 84 & Valens & $364-378$ & $E_{3}$ & $\notin$ & 17 & 2.07 & 5 & $\begin{array}{l}\text { DN VALEN-[S } \\
\text { PF AVG] }\end{array}$ & $\begin{array}{c}\text { SECVRITAS } \\
\text { [REIPVBLICAE] }\end{array}$ & $?$ & incerta & G133-134 & \\
\hline 85 & Valens & $364-378$ & $Æ_{3}$ & $\notin$ & 17 & 2.06 & 6 & $\begin{array}{l}\text { DN VALEN-S } \\
\text { PF [AVG] }\end{array}$ & $\begin{array}{c}\text { SECVRITAS } \\
\text { [REIPVBLICAE] }\end{array}$ & ? & incerta & G135-4 & \\
\hline 86 & $\begin{array}{l}\text { Valent. I/ } \\
\text { Valens }\end{array}$ & $364-378$ & Æ 3 & $\notin$ & 15 & 2.26 & 6 & DN VAL? & $\begin{array}{c}\text { SECVRITAS } \\
\text { [REIPVBLICAE] }\end{array}$ & $?$ & incerta & G134-130 & \\
\hline 87 & $\begin{array}{l}\text { Valent. I/ } \\
\text { Valens/Va- } \\
\text { lent. II }\end{array}$ & $364-378$ & $Æ_{3}$ & $A$ & 15 & 2.64 & 6 & DN VALEN? & $\begin{array}{c}\text { SECVRITAS } \\
\text { REI[PVBLICAE] }\end{array}$ & ? & incerta & G133-110 & \\
\hline 88 & $\begin{array}{l}\text { Valens/ } \\
\text { Gratian }\end{array}$ & $364-375$ & Æ 3 & $\notin$ & 14 & 2.42 & 11 & ? S PF AVG & $\begin{array}{l}\text { [GLORIA RO]- } \\
\text { MANOR[VM] }\end{array}$ & $--/ * / / ?$ & $\begin{array}{c}\mathrm{RIC} \\
96 / 176, \\
11 \mathrm{~b}-\mathrm{c} / 16 \mathrm{~b}\end{array}$ & G133-122 & \\
\hline 89 & $\begin{array}{l}\text { Valens/ } \\
\text { Gratian }\end{array}$ & $364-378$ & $\mathbb{E}_{3}$ & $A$ & 17 & 2.22 & 12 & ? & $\begin{array}{l}\text { GLORIA RO- } \\
\text { [MANORVM] }\end{array}$ & ? & incerta & G133-123 & \\
\hline 90 & Gratian & $367-378$ & $Æ_{3} 3$ & $\notin$ & 16 & 1.5 & 12 & $\begin{array}{l}\text { DN GRATIA- } \\
\text { NVS PF AVG }\end{array}$ & $\begin{array}{l}\text { [GLORIA RO- } \\
\text { MANORVM] }\end{array}$ & $?$ & incerta & G133-119 & \\
\hline 91 & Valentinian I & $367-375$ & Æ 3 & $\mathbb{E}$ & 18 & 2.14 & 6 & $\begin{array}{l}\text { DN VALENTI- } \\
\text { NI-ANVS PF } \\
\text { AVG }\end{array}$ & $\begin{array}{l}\text { GLORIA [RO]- } \\
\text { MANORVM }\end{array}$ & SM AQS & $\begin{array}{l}\text { RIC } 96 . \\
11 a\end{array}$ & G133-13 & \\
\hline 92 & Valentinian I & $367-375$ & $Æ_{3}$ & $A$ & 19 & 3.13 & 6 & $\begin{array}{l}\text { DN VALENTI- } \\
\text { NI-ANVS PF } \\
\text { AVG }\end{array}$ & $\begin{array}{l}\text { GLORIA RO-MA- } \\
\text { NORVM }\end{array}$ & $\begin{array}{l}\mathrm{Q} / * \mathrm{~K} / / \mathrm{B} \\
\mathrm{SISCV}\end{array}$ & $\begin{array}{l}\text { RIC 147 } \\
14 \mathrm{a}\end{array}$ & C25850 & \\
\hline 93 & Valentinian I & $367-375$ & $\star_{3} 3$ & $\notin$ & 18 & 2.02 & 1 & $\begin{array}{l}\text { DN VALENTI- } \\
\text { NI-ANVS PF } \\
\text { AVG }\end{array}$ & $\begin{array}{l}\text { GLORIA RO-MA- } \\
\text { NORVM }\end{array}$ & $\begin{array}{l}\mathrm{M} / * \mathrm{P} / / \mathrm{B} \\
\mathrm{SISC}\end{array}$ & $\begin{array}{c}\text { RIC 147, } \\
14 \mathrm{a}\end{array}$ & С31582 & \\
\hline 94 & Valentinian I & $367-375$ & $Æ_{3}$ & $\mathbb{E}$ & 18 & 2.85 & 5 & $\begin{array}{l}\text { DN VALENTI- } \\
\text { NI-ANVS PF } \\
\text { AVG }\end{array}$ & $\begin{array}{l}\text { GLORIA RO-MA- } \\
\text { NORVM }\end{array}$ & S/D B SIS C & $\begin{array}{l}\text { RIC 147 } \\
14 \mathrm{a}\end{array}$ & С 31583 & \\
\hline 95 & Valentinian I & $367-375$ & $\notin_{3} 3$ & E & 19 & 2.76 & 1 & $\begin{array}{l}\text { DN VALENTI- } \\
\text { NI-ANVS PF } \\
\text { AVG }\end{array}$ & $\begin{array}{l}\text { GLORIA RO-MA- } \\
\text { NORVM }\end{array}$ & $\begin{array}{c}--/ D / / *[B] \\
\text { SIS C }\end{array}$ & $\begin{array}{l}\text { RIC 147, } \\
14 \mathrm{a}\end{array}$ & G126-8 & \\
\hline 96 & Valentinian I & $367-375$ & $\notin_{3}$ & E & 17 & 2.04 & 1 & $\begin{array}{l}\text { DN VALENTI- } \\
\text { NI-ANVS PF } \\
\text { AVG }\end{array}$ & $\begin{array}{l}\text { GLORIA RO-MA- } \\
\text { NORVM }\end{array}$ & $\begin{array}{c}\mathrm{S} / * \mathrm{~F} / /[\mathrm{B} \\
\mathrm{SIS} \mathrm{C}]\end{array}$ & $\begin{array}{l}\text { RIC 147, } \\
\quad 14 \mathrm{a}\end{array}$ & G133-7 & \\
\hline 97 & Valentinian I & $367-375$ & $\notin_{3} 3$ & $\notin$ & 18 & 2.09 & 1 & $\begin{array}{l}\text { DN VALENTI- } \\
\text { NI-ANVS PF } \\
\text { AVG }\end{array}$ & $\begin{array}{l}\text { [GLORIA] RO- } \\
\text { MANORVM }\end{array}$ & $\begin{array}{c}\mathrm{F} / \mathrm{AK} / / \mathrm{B} \text { SIS } \\
\text { C4 }\end{array}$ & $\begin{array}{c}\text { RIC 147, } \\
14 \mathrm{a}\end{array}$ & G133-11 & \\
\hline 98 & Valentinian I & $367-375$ & $\notin_{3} 3$ & $\mathbb{E}$ & 18 & 2.15 & 1 & $\begin{array}{l}\text { DN VALENTI- } \\
\text { NI-ANVS PF } \\
\text { AVG }\end{array}$ & $\begin{array}{l}\text { GLORIA RO-MA- } \\
\text { NORVM }\end{array}$ & $\begin{array}{l}\mathrm{M} / * \mathrm{~F} / / \mathrm{B} \\
\mathrm{SISC}\end{array}$ & $\begin{array}{l}\text { RIC 147, } \\
14 \mathrm{a}\end{array}$ & G133-12 & \\
\hline 99 & Valentinian I & $367-375$ & $Æ_{3}$ & $\notin$ & 18 & 2.17 & 12 & $\begin{array}{c}\text { DN VALENTI- } \\
\text { NI-ANVS PF } \\
\text { AVG }\end{array}$ & $\begin{array}{l}\text { GLORIA RO-MA- } \\
\text { NORVM }\end{array}$ & $\begin{array}{l}\mathrm{M} / * \mathrm{~F} / / \mathrm{B} \\
\mathrm{SISC}\end{array}$ & $\begin{array}{l}\text { RIC 147 } \\
14 \mathrm{a}\end{array}$ & G133-27 & \\
\hline 100 & Valentinian I & $367-375$ & E 3 & $\notin$ & 17 & 2.51 & 1 & $\begin{array}{l}\text { DN VALENTI- } \\
\text { NI-ANVS PF } \\
\text { AVG }\end{array}$ & $\begin{array}{c}\text { GLORIA RO-MA- } \\
\text { NORVM }\end{array}$ & $\begin{array}{l}\mathrm{S} / * \mathrm{D} / / \mathrm{B} \\
\mathrm{SISC}\end{array}$ & $\begin{array}{c}\text { RIC } 147 . \\
14 \mathrm{a}\end{array}$ & G133-41 & \\
\hline 101 & Valentinian I & $367-375$ & Æ 3 & $\notin$ & 18 & 1.71 & 11 & $\begin{array}{c}\text { [DN } \\
\text { VALENTINI]- } \\
\text { ANVS PF AVG }\end{array}$ & $\begin{array}{l}\text { GLORIA RO- } \\
\text { [MANORVM] }\end{array}$ & $\begin{array}{c}--/ R / /[\cdot ? \\
\text { SIS C] }\end{array}$ & $\begin{array}{c}\text { RIC } 147 . \\
14 \mathrm{a}\end{array}$ & G133-74 & \\
\hline 102 & Valentinian I & $367-375$ & $\notin_{3} 3$ & $\notin$ & 18 & 2.5 & 7 & $\begin{array}{c}\text { [DN VALENT] } \\
\text { INI-ANVS PF } \\
\text { AVG }\end{array}$ & $\begin{array}{l}\text { [GLORIA] RO- } \\
\text { MANORVM }\end{array}$ & $\begin{array}{l}\text { S/D//B } \\
\text { SIS C }\end{array}$ & $\begin{array}{c}\text { RIC } 147 . \\
14 a\end{array}$ & G133-77 & \\
\hline
\end{tabular}




\begin{tabular}{|c|c|c|c|c|c|c|c|c|c|c|c|c|c|}
\hline \multicolumn{14}{|c|}{ DALJ } \\
\hline $\begin{array}{l}\text { CAT. } \\
\text { NO. }\end{array}$ & RULER & $\begin{array}{l}\text { DATING } \\
\text { (YRS) }\end{array}$ & DENOM. & MAT. & $\begin{array}{c}\text { D. } \\
(\mathrm{mm})\end{array}$ & $\begin{array}{l}\text { WT. } \\
\text { (g) }\end{array}$ & $\begin{array}{l}\text { ORI- } \\
\text { ENT. }\end{array}$ & OBVERSE & REVERSE & $\begin{array}{l}\text { MINT } \\
\text { MARKS }\end{array}$ & $\begin{array}{l}\text { REFE- } \\
\text { RENCE }\end{array}$ & $\begin{array}{l}\text { INV. } \\
\text { NO. }\end{array}$ & NOTE \\
\hline 103 & Valentinian I & $367-375$ & $\mathbb{E}_{3}$ & $\mathbb{E}$ & 18 & 2 & 5 & $\begin{array}{c}\text { DN VALENTI- } \\
\text { NI-ANVS PF } \\
\text { AVG }\end{array}$ & $\begin{array}{c}\text { GLORIA RO-MA- } \\
\text { NORVM }\end{array}$ & $--/ \mathrm{A} / / \mathrm{TES}$ & $\begin{array}{l}\text { RIC 178, } \\
26 \mathrm{a}\end{array}$ & G133-33 & \\
\hline 104 & Valentinian I & $367-375$ & E 3 & $\mathbb{E}$ & 18 & 2.09 & 1 & $\begin{array}{c}\text { DN VALENTI- } \\
\text { NI-ANVS PF } \\
\text { AVG }\end{array}$ & $\begin{array}{l}\text { GLORIA RO- } \\
\text { MANOR[VM] }\end{array}$ & ? & incerta & G133-99 & \\
\hline 105 & Valentinian I & $367-375$ & $\mathbb{E}_{3}$ & E & 18 & 2.36 & 5 & $\begin{array}{c}\text { DN VALENTI- } \\
\text { NI-ANVS PF } \\
\text { AVG }\end{array}$ & $\begin{array}{l}\text { SECVRITAS } \\
\text { REIPVBLICAE }\end{array}$ & $S M \downarrow R Q$ & $\begin{array}{l}\text { RIC 121, } \\
24 \mathrm{a}\end{array}$ & C 31585 & \\
\hline 106 & Valentinian I & $367-375$ & E 3 & $\mathbb{E}$ & 19 & 2.15 & 12 & $\begin{array}{c}\text { DN VALENTI- } \\
\text { NI-ANVS PF } \\
\text { AVG }\end{array}$ & $\begin{array}{l}\text { SECVRITAS } \\
\text { REIPVBLICAE }\end{array}$ & $R \cdot P R I M A$ & $\begin{array}{l}\text { RIC 121, } \\
24 \mathrm{a}\end{array}$ & G133-111 & \\
\hline 107 & Valentinian I & $367-375$ & $E_{3}$ & EE & 18 & 2.48 & 6 & $\begin{array}{c}\text { DN VALENTI- } \\
\text { NI-ANVS PF } \\
\text { AVG }\end{array}$ & $\begin{array}{l}\text { [SECVRITAS] } \\
\text { REIPVBLICAE }\end{array}$ & $\begin{array}{l}* \mathrm{D} / \mathrm{S} / / \Delta \\
\mathrm{SISC}\end{array}$ & $\begin{array}{l}\text { RIC 147, } \\
\text { 15a }\end{array}$ & G126-14 & \\
\hline 108 & Valentinian I & $367-375$ & $\mathbb{E}_{3}$ & 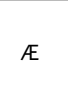 & 18 & 2.5 & 1 & $\begin{array}{c}\text { DN VALENTI- } \\
\text { NI-ANVS [PF } \\
\text { AVG] }\end{array}$ & $\begin{array}{l}\text { SECVRITAS } \\
\text { REIPVBLICAE }\end{array}$ & $\begin{array}{l}\mathrm{R} /--/ / \cdot A \\
\text { SIS C }\end{array}$ & $\begin{array}{l}\text { RIC 147, } \\
\text { 15a }\end{array}$ & G133-10 & \\
\hline 109 & Valentinian I & $367-375$ & $\mathbb{E}_{3}$ & $\mathbb{E}$ & 18 & 2.62 & 7 & $\begin{array}{c}\text { DN VALENTI- } \\
\text { NI-ANVS PF } \\
\text { AVG }\end{array}$ & $\begin{array}{l}\text { SECVRITAS } \\
\text { REIPVBLICAE }\end{array}$ & $\begin{array}{l}* \mathrm{P} / \mathrm{M} / / \Delta \\
\mathrm{SISC}\end{array}$ & $\begin{array}{l}\text { RIC 147, } \\
\text { 15a }\end{array}$ & G133-42 & \\
\hline 110 & Valentinian I & $367-375$ & Æ 3 & $\mathbb{E}$ & 18 & 2.14 & 6 & $\begin{array}{c}\text { DN VALENTI- } \\
\text { NI-ANVS PF } \\
\text { AVG }\end{array}$ & $\begin{array}{c}\text { SECVRITAS } \\
\text { [REIPVBLICAE] }\end{array}$ & $\begin{array}{c}--/ D / / * B \\
S I S C\end{array}$ & $\begin{array}{l}\text { RIC } 147 . \\
14 \mathrm{a}\end{array}$ & G133-45 & \\
\hline 111 & Valentinian I & $367-375$ & $\mathbb{E}_{3}$ & $\mathbb{E}$ & 18 & 1.8 & 11 & $\begin{array}{c}\text { [DN VALEN- } \\
\text { TINI-ANVS PF } \\
\text { AVG] }\end{array}$ & $\begin{array}{l}\text { SECVRITAS } \\
\text { REIPVBLICAE }\end{array}$ & $\begin{array}{c}\mathrm{M} / * \mathrm{P} / / \mathrm{B} \\
\mathrm{SISC}\end{array}$ & $\begin{array}{l}\text { RIC } 147 . \\
14 \mathrm{a}\end{array}$ & G133-50 & \\
\hline 112 & Valentinian I & $367-375$ & $\mathbb{E}_{3}$ & 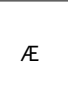 & 18 & 2.05 & 12 & $\begin{array}{c}\text { DN VALENTI- } \\
\text { NI-ANVS PF } \\
\text { AVG }\end{array}$ & $\begin{array}{l}\text { SECVRITAS } \\
\text { [REIPV]BLICAE }\end{array}$ & $\begin{array}{c}* \mathrm{P} / \mathrm{M} / / \Gamma \\
\mathrm{SISC}\end{array}$ & $\begin{array}{l}\text { RIC 147, } \\
\text { 15a }\end{array}$ & G133-54 & \\
\hline 113 & Valentinian I & $367-375$ & $\notin_{3} 3$ & $\mathbb{E}$ & 18 & 2.32 & 12 & $\begin{array}{c}\text { DN VALENTI- } \\
\text { NI-ANVS PF } \\
\text { AVG }\end{array}$ & $\begin{array}{c}\text { SECVRITAS } \\
\text { [REIPVBLICAE] }\end{array}$ & $\begin{array}{l}* \mathrm{P} / \mathrm{M} / / \Gamma \\
\mathrm{SISC}\end{array}$ & $\begin{array}{l}\text { RIC 147, } \\
\text { 15a }\end{array}$ & G133-55 & \\
\hline 114 & Valentinian I & $367-375$ & $E_{3}$ & $\mathbb{E}$ & 17 & 1.96 & 1 & $\begin{array}{c}\text { DN VALENTI- } \\
\text { NI-ANVS PF } \\
\text { AVG }\end{array}$ & $\begin{array}{l}\text { SECVRITAS } \\
\text { REIPVBLICAE }\end{array}$ & $\begin{array}{l}\mathrm{R} /--/ / \cdot A \\
\text { SIS C }\end{array}$ & $\begin{array}{l}\text { RIC 147, } \\
\text { 15a }\end{array}$ & G133-60 & \\
\hline 115 & Valentinian I & $367-375$ & $Æ_{3}$ & $\mathbb{E}$ & 18 & 1.86 & 1 & $\begin{array}{c}\text { [DN } \\
\text { VALENTINI]- } \\
\text { ANVS PF AVG }\end{array}$ & $\begin{array}{c}\text { SECVRITAS } \\
\text { REIPVBL[ICAE] }\end{array}$ & $\begin{array}{l}\text { D/--//?A } \\
\text { SIS C }\end{array}$ & $\begin{array}{l}\text { RIC 147, } \\
\text { 15a }\end{array}$ & G133-70 & \\
\hline 116 & Valentinian I & $367-375$ & E3 & $\notin E$ & 17 & 2.27 & 7 & $\begin{array}{c}\text { [DN } \\
\text { VALENTINI]- } \\
\text { ANVS PF AVG }\end{array}$ & $\begin{array}{l}\text { [SECVRITAS] } \\
\text { REIPVBLICAE }\end{array}$ & $\begin{array}{l}\mathrm{R} /--/ / \cdot \Delta \\
\operatorname{SISC}\end{array}$ & $\begin{array}{l}\text { RIC 147, } \\
\text { 15a }\end{array}$ & G133-96 & \\
\hline 117 & Valentinian I & $367-375$ & $Æ_{3} 3$ & E & 18 & 2.22 & 12 & $\begin{array}{c}\text { DN } \\
\text { VALENT[INI]- } \\
\text { ANVS P[F } \\
\text { AVG] }\end{array}$ & $\begin{array}{c}\text { [SECVR]ITAS } \\
\text { [REIPVBLICAE] }\end{array}$ & $\begin{array}{c}* \mathrm{P} /[\mathrm{M}] / / \text { ? } \\
\mathrm{SIS} \mathrm{C}\end{array}$ & $\begin{array}{l}\text { RIC 147, } \\
\text { 15a }\end{array}$ & G133-108 & \\
\hline 118 & Valentinian I & $367-375$ & $\mathbb{E}_{3}$ & EE & 19 & 2.24 & 6 & $\begin{array}{c}\text { DN VALENTI- } \\
\text { NI-ANVS PF } \\
\text { AVG }\end{array}$ & $\begin{array}{c}\text { SECVRITAS } \\
\text { REIPVBLICAE }\end{array}$ & SM N A & $\begin{array}{l}\text { RIC } 252 \\
12 \mathrm{a}\end{array}$ & G133-135 & \\
\hline 119 & $\begin{array}{l}\text { Valent. I/ } \\
\text { Valens }\end{array}$ & $367-375$ & $E_{3}$ & E & 18 & 2.49 & 11 & $\begin{array}{l}\text { [DN V]ALEN } \\
\text { ? AVG }\end{array}$ & $\begin{array}{l}\text { [GL]ORIA RO- } \\
\text { MANORVM }\end{array}$ & $S M A Q P$ & $\begin{array}{l}\text { RIC 96, } \\
11 a / b\end{array}$ & G133-125 & \\
\hline 120 & Valens & $367-375$ & $E_{3}$ & E & 18 & 1.34 & 6 & $\begin{array}{c}\text { [DN V]ALEN-S } \\
\text { PF AVG }\end{array}$ & $\begin{array}{l}\text { GL[ORIA RO-] } \\
\text { MANORVM }\end{array}$ & $S M A Q P$ & $\begin{array}{l}\text { RIC } 96 \\
11 \mathrm{~b}\end{array}$ & G133-91 & \\
\hline 121 & Valens & $367-375$ & $E_{3}$ & E & 18 & 2.04 & 5 & $\begin{array}{c}\text { [DN] VALEN-S } \\
\text { PF AVG }\end{array}$ & $\begin{array}{c}\text { [GLORIA RO-M] } \\
\text { ANORVM }\end{array}$ & $\begin{array}{c}--/ * / / S M \\
\text { AQP }\end{array}$ & $\begin{array}{l}\text { RIC 96, } \\
\text { 11b }\end{array}$ & G133-129 & \\
\hline 122 & Valens & $367-375$ & $E_{3}$ & EE & 18 & 2.23 & 6 & $\begin{array}{l}\text { DN VALEN-S } \\
\text { PF AVG }\end{array}$ & $\begin{array}{c}\text { GLORIA RO-MA- } \\
\text { NORVM }\end{array}$ & $\begin{array}{l}--/ R / / \cdot B \\
\text { SIS C }\end{array}$ & $\begin{array}{l}\text { RIC 147, } \\
14 \mathrm{~b}\end{array}$ & G126-4 & \\
\hline 123 & Valens & $367-375$ & $E_{3}$ & E & 18 & 1.65 & 7 & $\begin{array}{l}\text { DN VALEN-S } \\
\text { PF AVG }\end{array}$ & $\begin{array}{c}\text { GLORIA RO-MA- } \\
\text { NORVM }\end{array}$ & $\begin{array}{l}--/ R / / \cdot B \\
\text { SIS C }\end{array}$ & $\begin{array}{l}\text { RIC 147, } \\
14 \mathrm{~b}\end{array}$ & G133-15 & \\
\hline 124 & Valens & $367-375$ & $E_{3}$ & E & 18 & 3.32 & 12 & $\begin{array}{l}\text { DN VALEN-S } \\
\text { PF AVG }\end{array}$ & $\begin{array}{l}\text { GLORIA RO-MA- } \\
\text { NORVM }\end{array}$ & $\begin{array}{c}--/ R / / \cdot B \\
\text { SIS C }\end{array}$ & $\begin{array}{l}\text { RIC 147, } \\
14 \mathrm{~b}\end{array}$ & G133-19 & \\
\hline 125 & Valens & $367-375$ & E 3 & E & 18 & 2.19 & 6 & $\begin{array}{l}\text { DN VALEN-S } \\
\text { PF AVG }\end{array}$ & $\begin{array}{l}\text { GLORIA RO-MA- } \\
\text { NORVM }\end{array}$ & {$[*\lceil S I S C]$} & $\begin{array}{l}\text { RIC 147, } \\
14 \mathrm{~b}\end{array}$ & G133-30 & \\
\hline 126 & Valens & $367-375$ & Æ 3 & E & 17 & 2.35 & 12 & $\begin{array}{l}\text { DN VALEN-S } \\
\text { PF AVG }\end{array}$ & $\begin{array}{l}\text { GLORIA RO- } \\
\text { [MANORVM] }\end{array}$ & $\begin{array}{l}--/ R / / \cdot \Gamma \\
\text { SIS C }\end{array}$ & $\begin{array}{l}\text { RIC 147, } \\
14 \mathrm{~b}\end{array}$ & G133-37 & \\
\hline
\end{tabular}




\begin{tabular}{|c|c|c|c|c|c|c|c|c|c|c|c|c|c|}
\hline \multicolumn{14}{|c|}{ DALJ } \\
\hline $\begin{array}{l}\text { CAT. } \\
\text { NO. }\end{array}$ & RULER & $\begin{array}{c}\text { DATING } \\
\text { (YRS) }\end{array}$ & DENOM. & MAT. & $\begin{array}{c}\text { D. } \\
(\mathrm{mm})\end{array}$ & $\begin{array}{l}\text { WT. } \\
\text { (g) }\end{array}$ & $\begin{array}{l}\text { ORI- } \\
\text { ENT. }\end{array}$ & OBVERSE & REVERSE & $\begin{array}{l}\text { MINT } \\
\text { MARKS }\end{array}$ & $\begin{array}{l}\text { REFE- } \\
\text { RENCE }\end{array}$ & $\begin{array}{l}\text { INV. } \\
\text { NO. }\end{array}$ & NOTE \\
\hline 127 & Valens & $367-375$ & $Æ_{3}$ & $\mathscr{E}$ & 18 & 2.55 & 1 & $\begin{array}{l}\text { DN VALEN-S } \\
\text { PF AVG }\end{array}$ & $\begin{array}{c}\text { GLORIA RO-MA- } \\
\text { NORVM }\end{array}$ & $\begin{array}{l}--/ R / / \cdot B \\
\text { SIS C }\end{array}$ & $\begin{array}{c}\text { RIC 147 } \\
14 \mathrm{~b}\end{array}$ & G133-51 & \\
\hline 128 & Valens & $367-375$ & $\notin_{3}$ & E & 18 & 2.15 & 6 & $\begin{array}{l}\text { DN [VALEN-S] } \\
\text { PF AVG }\end{array}$ & $\begin{array}{l}\text { GLORIA RO- } \\
\text { [MANORVM] }\end{array}$ & $\begin{array}{c}--/ D / / * \Gamma \\
\text { SIS C }\end{array}$ & $\begin{array}{c}\text { RIC 147 } \\
14 \mathrm{~b}\end{array}$ & G133-58 & \\
\hline 129 & Valens & $367-375$ & $\notin_{3}$ & E & 17 & 2.16 & 1 & $\begin{array}{l}\text { DN VALEN-S } \\
\text { PF AVG }\end{array}$ & $\begin{array}{c}\text { GLORIA RO-MA- } \\
\text { NORVM }\end{array}$ & $\begin{array}{c}--/ D / / * \Gamma \\
\text { SIS C }\end{array}$ & $\begin{array}{c}\text { RIC 147 } \\
14 \mathrm{~b}\end{array}$ & G133-67 & \\
\hline 130 & Valens & $367-375$ & $\notin_{3}$ & E & 18 & 1.92 & 1 & $\begin{array}{l}\text { DN VALEN-S } \\
\text { PF AVG }\end{array}$ & $\begin{array}{l}\text { GLORIA RO-MA- } \\
\text { NORVM }\end{array}$ & $\begin{array}{c}--/ D / / * \Gamma \\
\text { SIS C }\end{array}$ & $\begin{array}{c}\text { RIC 147 } \\
14 \mathrm{~b}\end{array}$ & G135-1 & \\
\hline 131 & Valens & $367-375$ & Æ 3 & FE & 17 & 2.01 & 11 & $\begin{array}{c}\text { DN VALEN-[S } \\
\text { PF AVG] }\end{array}$ & $\begin{array}{c}\text { [GLORIA RO-MA] } \\
\text { NORVM }\end{array}$ & ?/B//[T]ES & $\begin{array}{c}\text { RIC } 178 \\
26 a\end{array}$ & G133-57 & \\
\hline 132 & Valens & $367-375$ & $\notin_{3}$ & E & 18 & 2.95 & 6 & $\begin{array}{l}\text { DN VAL[ENS] } \\
\text { PF AVG }\end{array}$ & $\begin{array}{c}\text { GLORIA RO-MA- } \\
\text { NORVM }\end{array}$ & CONS4 & $\begin{array}{l}\text { RIC 220 } \\
41 \mathrm{~b}\end{array}$ & C23487 & \\
\hline 133 & Valens & $367-375$ & $\mathbb{E}_{3}$ & E & 18 & 1.85 & 6 & $\begin{array}{l}\text { DN VALEN-S } \\
\text { PF AVG }\end{array}$ & $\begin{array}{l}\text { [GLORIA RO- } \\
\text { MANORVM] }\end{array}$ & $?$ & incerta & G133-9 & \\
\hline 134 & Valens & $367-375$ & $Æ_{3} 3$ & $E$ & 18 & 2.55 & 7 & $\begin{array}{l}\text { DN VALEN-S } \\
\text { PF AVG }\end{array}$ & $\begin{array}{l}\text { SECVRI[T]AS } \\
\text { REIPVBLICAE }\end{array}$ & $S M A Q P$ & $\begin{array}{l}\text { RIC 96, } \\
12 \mathrm{~b}\end{array}$ & С31587 & \\
\hline 135 & Valens & $367-375$ & $\notin_{3} 3$ & E & 20 & 2.85 & 6 & $\begin{array}{l}\text { DN VALEN-[S } \\
\text { PF AVG] }\end{array}$ & $\begin{array}{l}\text { SECVRITAS } \\
\text { REIPVBLICAE }\end{array}$ & $\begin{array}{l}\text { I. wre- } \\
\text { ath/--//SM } \\
\text { AQP }\end{array}$ & $\begin{array}{l}\text { RIC 96, } \\
12 \mathrm{~b}\end{array}$ & G133-34 & \\
\hline 136 & Valens & $367-375$ & Æ 3 & E & 18 & 2.43 & 5 & $\begin{array}{l}\text { DN VALEN-S } \\
\text { PF AVG }\end{array}$ & $\begin{array}{l}\text { SECVRITAS } \\
\text { REIPVBLICAE }\end{array}$ & SM AQS & $\begin{array}{l}\text { RIC 96, } \\
12 \mathrm{~b}\end{array}$ & G133-43 & \\
\hline 137 & Valens & $367-375$ & $\notin_{3}$ & $E$ & 18 & 3 & 1 & $\begin{array}{l}\text { DN VALEN-S } \\
\text { PF AVG }\end{array}$ & $\begin{array}{l}\text { SECVRITAS } \\
\text { REIPVBLICAE }\end{array}$ & R.QVARTA & $\begin{array}{l}\text { RIC 96, } \\
24 \mathrm{~b}\end{array}$ & G133-80 & \\
\hline 138 & Valens & $367-375$ & $Æ 3$ & E & 18 & 1.5 & 7 & $\begin{array}{l}\text { [DN VAL]EN-S } \\
\text { PF AVG }\end{array}$ & $\begin{array}{l}\text { [SECVRITAS REI] } \\
\text { PVBLICAE }\end{array}$ & SM AQS & RIC $95,9 b$ & G133-88 & \\
\hline 139 & Valens & $367-375$ & $\notin_{3}$ & $E$ & 14 & 1.68 & 5 & $\begin{array}{c}\text { [DN VA]LEN-S } \\
\text { P[F AVG] }\end{array}$ & $\begin{array}{l}\text { SECV[RITAS } \\
\text { REIPVBLICAE] }\end{array}$ & $R \cdot \operatorname{PRIM}[A]$ & $\begin{array}{l}\text { RIC 121, } \\
24 \mathrm{~b}\end{array}$ & G126-20 & \\
\hline 140 & Valens & $367-375$ & $Æ_{3} 3$ & E & 18 & 1.84 & 6 & $\begin{array}{l}\text { [DN VALEN]-S } \\
\text { PF AVG }\end{array}$ & $\begin{array}{l}\text { [SECV]RITAS } \\
\text { [REI]PVBLICAE }\end{array}$ & $R \cdot$ TERTIA & $\begin{array}{l}\text { RIC 121, } \\
24 \mathrm{~b}\end{array}$ & G133-112 & \\
\hline 141 & Valens & $367-375$ & $\notin_{3} 3$ & E & 18 & 2.04 & 11 & $\begin{array}{l}\text { DN VALEN-S } \\
\text { PF AVG }\end{array}$ & $\begin{array}{l}\text { [SECVRITAS] } \\
\text { REIPVBLICAE }\end{array}$ & $R \cdot$ QVARTA & $\begin{array}{l}\text { RIC 121, } \\
24 \mathrm{~b}\end{array}$ & G133-31 & \\
\hline 142 & Valens & $367-375$ & $E_{3}$ & EE & 19 & 2.12 & 1 & $\begin{array}{l}\text { DN VALEN-S } \\
\text { PF AVG }\end{array}$ & $\begin{array}{l}\text { SECVRITAS } \\
\text { REIPVBLICAE }\end{array}$ & $\begin{array}{l}{ }^{* F / M} / / \mathrm{A} \\
\text { SIS C }\end{array}$ & $\begin{array}{l}\text { RIC 147, } \\
\text { 15b }\end{array}$ & С31590 & \\
\hline 143 & Valens & $367-375$ & $\notin_{3} 3$ & $E$ & 18 & 2.43 & 6 & $\begin{array}{l}\text { DN VALEN-[S } \\
\text { PF AVG] }\end{array}$ & $\begin{array}{c}\text { SECVRITAS } \\
\text { R[EIPVBLICAE] }\end{array}$ & $\begin{array}{l}\text { D/--//*A } \\
\text { SIS C }\end{array}$ & $\begin{array}{l}\text { RIC 147, } \\
15 \mathrm{~b}\end{array}$ & G126-6 & \\
\hline 144 & Valens & $367-375$ & $\notin_{3} 3$ & EE & 17 & 2.5 & 1 & $\begin{array}{l}\text { DN VALEN-S } \\
\text { PF AVG }\end{array}$ & $\begin{array}{l}\text { SECVRITAS } \\
\text { REIPVBLICAE }\end{array}$ & $\begin{array}{l}\mathrm{R} /--/ / * \mathrm{~A} \\
\mathrm{SISC}\end{array}$ & $\begin{array}{l}\text { RIC 147, } \\
\text { 15b }\end{array}$ & G126-7 & \\
\hline 145 & Valens & $367-375$ & $\notin_{3}$ & E & 18 & 2.38 & 11 & $\begin{array}{l}\text { DN VALEN-S } \\
\text { PF AVG }\end{array}$ & $\begin{array}{l}\text { SECVRITAS } \\
\text { REIPVBLICAE }\end{array}$ & $\begin{array}{l}* \mathrm{P} / \mathrm{M} / / \mathrm{A} \\
\text { SIS C }\end{array}$ & $\begin{array}{l}\text { RIC 147, } \\
15 \mathrm{~b}\end{array}$ & G128-1 & \\
\hline 146 & Valens & $367-375$ & $\notin_{3}$ & EE & 17 & 1.92 & 5 & $\begin{array}{l}\text { DN VALEN-S } \\
\text { PF AVG }\end{array}$ & $\begin{array}{l}\text { SECVRITAS } \\
\text { REIPVBLICAE }\end{array}$ & $\begin{array}{l}* \mathrm{~K} / \mathrm{Q} / / \mathrm{A} \\
\mathrm{SISCR}\end{array}$ & $\begin{array}{l}\text { RIC 147, } \\
\text { 15b }\end{array}$ & G133-18 & \\
\hline 147 & Valens & $367-375$ & $\notin_{3} 3$ & E & 18 & 2.31 & 6 & $\begin{array}{l}\text { DN VALEN-S } \\
\text { PF AVG }\end{array}$ & $\begin{array}{l}\text { SECVRITAS } \\
\text { REIPVBLICAE }\end{array}$ & $\begin{array}{c}* \mathrm{RO} / \mathrm{M} / / \mathrm{A} \\
\mathrm{SISCR}\end{array}$ & $\begin{array}{c}\text { RIC 147, } \\
15 \mathrm{~b}\end{array}$ & G133-20 & \\
\hline 148 & Valens & $367-375$ & $\notin_{3}$ & E & 18 & 2.13 & 12 & $\begin{array}{l}\text { [DN VALEN]-S } \\
\text { PF AVG }\end{array}$ & $\begin{array}{c}\text { SECVRITAS } \\
\text { [REIPVB]LICAE }\end{array}$ & $\begin{array}{l}\text { *F/M } / / A \\
\text { SIS C }\end{array}$ & $\begin{array}{l}\text { RIC 147, } \\
\text { 15b }\end{array}$ & G133-28 & \\
\hline 149 & Valens & $367-375$ & $\notin_{3}$ & EE & 18 & 2.27 & 6 & $\begin{array}{l}\text { DN VALEN-S } \\
\text { PF AVG }\end{array}$ & $\begin{array}{l}\text { SECVRITAS } \\
\text { REIPVBLICA[E] }\end{array}$ & A SIS C & $\begin{array}{l}\text { RIC 147, } \\
15 \mathrm{~b}\end{array}$ & G133-36 & \\
\hline 150 & Valens & $367-375$ & $\notin_{3}$ & E & 19 & 2.25 & 2 & $\begin{array}{l}\text { DN VALEN-S } \\
\text { PF AVG }\end{array}$ & $\begin{array}{c}\text { SECVRITAS } \\
\text { REIPVBLI[CAE] }\end{array}$ & $\begin{array}{l}* \mathrm{P} / \mathrm{M} / /[\mathrm{A}] \\
\mathrm{SISC}\end{array}$ & $\begin{array}{c}\text { RIC 147, } \\
15 \mathrm{~b}\end{array}$ & G133-40 & \\
\hline 151 & Valens & $367-375$ & $\notin_{3}$ & E & 18 & 2.11 & 1 & $\begin{array}{l}\text { DN VALEN-S } \\
\text { PF AVG }\end{array}$ & $\begin{array}{l}\text { SECVRITAS } \\
\text { REIPVBLICAE }\end{array}$ & $\begin{array}{l}* \mathrm{~F} / \mathrm{M} / / \mathrm{A} \\
\text { SIS C }\end{array}$ & $\begin{array}{c}\text { RIC 147, } \\
15 \mathrm{~b}\end{array}$ & G133-53 & \\
\hline 152 & Valens & $367-375$ & $\notin_{3}$ & E & 19 & 2.3 & 6 & $\begin{array}{l}\text { DN VAL[EN]-S } \\
\text { PF AVG }\end{array}$ & $\begin{array}{l}\text { SEC[VRITAS RE] } \\
\text { IPVBLICAE }\end{array}$ & $\begin{array}{c}* \mathrm{~F} / \mathrm{M} / /[\mathrm{A} \\
\mathrm{SIS} \mathrm{C}]\end{array}$ & $\begin{array}{c}\text { RIC 147, } \\
15 \mathrm{~b}\end{array}$ & G133-98 & \\
\hline 153 & Valens & $367-375$ & $\notin_{3}$ & $E$ & 18 & 2.31 & 6 & $\begin{array}{l}\text { DN VALEN-S } \\
\text { PF AVG }\end{array}$ & $\begin{array}{l}\text { SECVRITAS } \\
\text { REIPVBLICAE }\end{array}$ & $\begin{array}{c}* \mathrm{RO} / \cdot \mathrm{M} / / \mathrm{A} \\
\mathrm{SISC}\end{array}$ & $\begin{array}{c}\text { RIC 147, } \\
15 \mathrm{~b}\end{array}$ & G133-62 & \\
\hline 154 & Valens & $367-375$ & $\notin_{3} 3$ & E & 18 & 1.96 & 12 & $\begin{array}{l}\text { DN VALEN-S } \\
\text { PF AVG }\end{array}$ & $\begin{array}{l}\text { SECVRITAS } \\
\text { REIPVBLICAE }\end{array}$ & $\begin{array}{c}* \mathrm{P} / \cdot \mathrm{M} / / \mathrm{A} \\
\mathrm{SISC}\end{array}$ & $\begin{array}{c}\text { RIC 147, } \\
15 \mathrm{~b}\end{array}$ & G133-69 & \\
\hline 155 & Valens & $367-375$ & $\notin_{3} 3$ & E & 18 & 2.53 & 2 & $\begin{array}{l}\text { DN VALEN-S } \\
\text { PF AVG }\end{array}$ & $\begin{array}{l}\text { SECVRITAS } \\
\text { REIPVBLICAE }\end{array}$ & $\begin{array}{l}\mathrm{R} /--/ / \cdot \mathrm{A} \\
\mathrm{SISC}\end{array}$ & $\begin{array}{c}\text { RIC 147, } \\
15 \mathrm{~b}\end{array}$ & G133-72 & \\
\hline 156 & Valens & $367-375$ & $\notin_{3} 3$ & E & 17 & 1.72 & 2 & $\begin{array}{l}\text { DN VALEN-S } \\
\text { PF AVG }\end{array}$ & $\begin{array}{l}\text { SECVRITAS } \\
\text { REIPVBLICAE }\end{array}$ & $\begin{array}{c}* \mathrm{~F} / \mathrm{S} / / \mathrm{A} \\
\mathrm{SISC}\end{array}$ & $\begin{array}{c}\text { RIC 147, } \\
15 \mathrm{~b}\end{array}$ & G133-79 & \\
\hline
\end{tabular}




\begin{tabular}{|c|c|c|c|c|c|c|c|c|c|c|c|c|c|}
\hline \multicolumn{14}{|c|}{ DALJ } \\
\hline $\begin{array}{l}\text { CAT. } \\
\text { NO. }\end{array}$ & RULER & $\begin{array}{l}\text { DATING } \\
\text { (YRS) }\end{array}$ & DENOM. & MAT. & $\begin{array}{c}\text { D. } \\
(\mathrm{mm})\end{array}$ & $\begin{array}{l}\text { WT. } \\
(\mathrm{g})\end{array}$ & $\begin{array}{l}\text { ORI- } \\
\text { ENT. }\end{array}$ & OBVERSE & REVERSE & $\begin{array}{l}\text { MINT } \\
\text { MARKS }\end{array}$ & $\begin{array}{l}\text { REFE- } \\
\text { RENCE }\end{array}$ & $\begin{array}{l}\text { INV. } \\
\text { NO. }\end{array}$ & NOTE \\
\hline 157 & Valens & $367-375$ & Æ3 & $\notin$ & 17 & 1.96 & 7 & $\begin{array}{l}\text { DN VALEN-S } \\
\text { PF AVG }\end{array}$ & $\begin{array}{c}\text { SECVRITAS } \\
\text { [REIPVBLICA]E }\end{array}$ & $\begin{array}{l}* \mathrm{P} / \mathrm{M} / / \mathrm{A} \\
\mathrm{SISC}\end{array}$ & $\begin{array}{l}\text { RIC 147, } \\
\text { 15b }\end{array}$ & G133-85 & \\
\hline 158 & Valens & $367-375$ & $\mathbb{E}_{3}$ & E & 18 & 1.66 & 1 & $\begin{array}{l}\text { DN VALEN-S } \\
\text { PF AVG }\end{array}$ & $\begin{array}{l}\text { [SECVRITAS] } \\
\text { REIPVBLICAE }\end{array}$ & $\begin{array}{l}* \mathrm{~K} / \mathrm{Q} / / \mathrm{A} \\
\mathrm{SISCR}\end{array}$ & $\begin{array}{l}\text { RIC 147, } \\
15 \mathrm{~b}\end{array}$ & G133-92 & \\
\hline 159 & Valens & $367-375$ & $\mathbb{E}_{3}$ & E & 17 & 1.98 & 7 & $\begin{array}{l}\text { DN VALEN-[S] } \\
\text { PF AVG }\end{array}$ & $\begin{array}{l}\text { SECVRITAS [REI] } \\
\text { PVBLICAE }\end{array}$ & $\begin{array}{l}\text { D/S//A } \\
\text { SIS C }\end{array}$ & $\begin{array}{l}\text { RIC 147, } \\
15 \mathrm{~b}\end{array}$ & G134-1 & \\
\hline 160 & Valens & $367-375$ & E 3 & E & 19 & 2.23 & 7 & $\begin{array}{c}\text { DN VALEN-S } \\
\text { [PF AVG] }\end{array}$ & $\begin{array}{l}\text { SECVRITAS } \\
\text { REIPVBLICAE }\end{array}$ & ${ }^{*}$ A S SIS C & $\begin{array}{l}\text { RIC 147, } \\
15 \mathrm{~b}\end{array}$ & G133-49 & \\
\hline 161 & Valens & $367-375$ & $\mathbb{E}_{3}$ & E & 18 & 3.01 & 6 & $\begin{array}{l}\text { DN VALEN-S } \\
\text { PF AVG }\end{array}$ & $\begin{array}{c}\text { SECVRITAS } \\
\text { [REIPVBLICAE] }\end{array}$ & $\mathrm{P} / \Delta / /[\mathrm{TES}]$ & $\begin{array}{l}\text { RIC 178, } \\
27 \mathrm{~b}\end{array}$ & G134-3 & \\
\hline 162 & Gratian & $367-375$ & $\mathbb{E}_{3}$ & E & 18 & 2.85 & 1 & $\begin{array}{l}\text { DN GRATIA- } \\
\text { NVS PF AVG }\end{array}$ & $\begin{array}{l}\text { GLORIA RO-[M] } \\
\text { ANORVM }\end{array}$ & $\Delta \mathrm{SISC}$ & $\begin{array}{l}\text { RIC 147, } \\
14 \mathrm{C}\end{array}$ & G126-12 & \\
\hline 163 & Gratian & $367-375$ & E 3 & E & 18 & 2.53 & 12 & $\begin{array}{l}\text { DN GRATIA- } \\
\text { NVS PF AVG }\end{array}$ & $\begin{array}{c}\text { GLORIA RO-MA- } \\
\text { NORVM }\end{array}$ & $\begin{array}{l}\mathrm{S} / * \mathrm{~F} / / \Gamma \\
\mathrm{SISC}\end{array}$ & $\begin{array}{l}\text { RIC 147, } \\
14 \mathrm{C}\end{array}$ & G133-3 & \\
\hline 164 & Gratian & $367-375$ & $\mathbb{E}_{3}$ & E & 18 & 3.29 & 6 & $\begin{array}{l}\text { DN GRATIA- } \\
\text { NVS PF AVG }\end{array}$ & $\begin{array}{c}\text { GLORIA RO-MA- } \\
\text { NORVM }\end{array}$ & $\begin{array}{c}--/ D / / * B \\
\text { SIS C }\end{array}$ & $\begin{array}{l}\text { RIC 147, } \\
14 \mathrm{C}\end{array}$ & G133-52 & \\
\hline 165 & Gratian & $367-375$ & $Æ_{3} 3$ & $\mathbb{E}$ & 18 & 2.29 & 2 & $\begin{array}{l}\text { DN GRATIA- } \\
\text { NVS PF AVG }\end{array}$ & $\begin{array}{c}\text { GLORIA RO-MA- } \\
\text { NORVM }\end{array}$ & $\begin{array}{l}--/ R / / \cdot B \\
\text { SIS C }\end{array}$ & $\begin{array}{l}\text { RIC 147, } \\
\quad 14 \mathrm{C}\end{array}$ & G133-81 & \\
\hline 166 & Gratian & $367-375$ & $\mathbb{E}_{3}$ & E & 18 & 1.72 & 1 & $\begin{array}{l}\text { DN GRATIA- } \\
\text { NVS PF [AVG] }\end{array}$ & $\begin{array}{l}\text { [GL]ORIA RO- } \\
\text { MANORVM }\end{array}$ & $\begin{array}{c}\text { S·/RÁ//[L] } \\
\text { SIS C E }\end{array}$ & $\begin{array}{l}\text { RIC 147, } \\
14 \mathrm{C}\end{array}$ & G137-4 & \\
\hline 167 & Gratian & $367-375$ & $\mathbb{E}_{3}$ & E & 17 & 2.44 & 12 & $\begin{array}{l}\text { DN GRATIA- } \\
\text { NVS PF AVG }\end{array}$ & $\begin{array}{c}\text { GLORIA RO-MA- } \\
\text { NORVM }\end{array}$ & $* / * \mathrm{~F} / / \mathrm{TES}$ & $\begin{array}{l}\text { RIC 178, } \\
26 \mathrm{c}\end{array}$ & G133-64 & \\
\hline 168 & Gratian & $367-375$ & Æ3 & E & 16 & 2.09 & 11 & $\begin{array}{l}\text { [DN GRATIA- } \\
\text { NV]S PF AVG }\end{array}$ & $\begin{array}{l}\text { [GLORIA RO]- } \\
\text { MANORVM }\end{array}$ & $--/ \mathrm{B} / / \mathrm{TES}$ & $\begin{array}{l}\text { RIC } 178, \\
26 \mathrm{c}\end{array}$ & G133-8 & \\
\hline 169 & Gratian & $367-375$ & $\mathbb{E}_{3}$ & E & 17 & 2.48 & 6 & $\begin{array}{l}\text { DN GRATIA- } \\
\text { NVS PF AVG }\end{array}$ & $\begin{array}{l}\text { SECVRITAS } \\
\text { REIPVBLICAE }\end{array}$ & R.QVARTA & $\begin{array}{l}\text { RIC 121, } \\
24 \mathrm{C}\end{array}$ & G128-2 & \\
\hline 170 & $\begin{array}{l}\text { Valent. I- } \\
\text { Gratian }\end{array}$ & $367-375$ & E 3 & E & 15 & 2.77 & 5 & $?$ & $\begin{array}{l}\text { [SECVRITAS } \\
\text { REIPV]BLICAE }\end{array}$ & $\begin{array}{l}{[\mathrm{R} \cdot \mathrm{SEC}]} \\
\mathrm{VNDA}\end{array}$ & $\begin{array}{l}\text { RIC 121, } \\
24 \mathrm{a}-\mathrm{c}\end{array}$ & G133-106 & \\
\hline 171 & Valens & $367-378$ & E 3 & $\mathbb{E}$ & 19 & 2.48 & 12 & $\begin{array}{l}\text { DN VALEN-S } \\
\text { PF AVG }\end{array}$ & $\begin{array}{l}\text { SECVRITAS } \\
\text { REIPVBLICAE }\end{array}$ & $S M \downarrow R P$ & $\begin{array}{c}\text { RIC } \\
121 / 122 \\
24 \mathrm{~b} / 28 \mathrm{a}\end{array}$ & С31589 & $\begin{array}{l}\text { issues } \\
\text { indi- } \\
\text { stingu- } \\
\text { ishable }\end{array}$ \\
\hline 172 & Valentinian II & $378-383$ & Æ 2 & E & 24 & 3.71 & 5 & $\begin{array}{c}\text { DN VALENTI- } \\
\text { NIANVS [IVN } \\
\text { PF AVG] }\end{array}$ & $\begin{array}{l}\text { REPARATIO } \\
\text { REI[PVB] }\end{array}$ & *A SIS C & $\begin{array}{l}\text { RIC 150, } \\
26 \mathrm{~b}\end{array}$ & G133-39 & \\
\hline 173 & Valentinian II & $378-383$ & $\mathbb{E} 2_{2}$ & $\mathbb{E}$ & 24 & 4.6 & 6 & $\begin{array}{c}\text { DN VALENTI- } \\
\text { NIANVS IVN } \\
\text { PF AVG }\end{array}$ & $\begin{array}{l}\text { REPARATIO } \\
\text { REIPVB }\end{array}$ & $*$ B SIS C & $\begin{array}{l}\text { RIC 150, } \\
26 \mathrm{~b}\end{array}$ & G133-47 & \\
\hline 174 & Theodosius I & $379-383$ & $\notin 4$ & $\mathbb{E}$ & 15 & 1.61 & 12 & $\begin{array}{l}\text { DN THEODO- } \\
\text { SIVS PF AVG }\end{array}$ & VOT/V|MVL[T]|X & B SIS C & $\begin{array}{l}\text { RIC 152, } \\
29 \mathrm{~b}\end{array}$ & G126-11 & \\
\hline 175 & Theodosius I & $379-383$ & ÆE 4 & E & 15 & 1.61 & 12 & $\begin{array}{l}\text { DN THEODO- } \\
\text { SIVS PF AVG }\end{array}$ & $\begin{array}{l}\text { VOT[|X|] } \\
\text { MVLT|X[X] }\end{array}$ & A SIS C & $\begin{array}{l}\text { RIC 152, } \\
30 \mathrm{~b}\end{array}$ & G126-15 & \\
\hline 176 & Aelia Flaccilla & $383-384 ?$ & $\notin 2$ & E & 24 & 4.24 & 1 & $\begin{array}{l}\text { AEL FLAC- } \\
\text { CILLA AVG }\end{array}$ & $\begin{array}{c}\text { SALVS REI-PVBLI- } \\
\text { CAE }\end{array}$ & B SIS C. & $\begin{array}{c}\text { RIC 153, } \\
34\end{array}$ & G133-1 & \\
\hline 177 & Theodosius I & $383-384 ?$ & Æ 2 & E & 22 & 4.12 & 12 & $\begin{array}{l}\text { DN THEODO- } \\
\text { SIVS PF AVG }\end{array}$ & $\begin{array}{c}\text { GLORIA RO-MA- } \\
\text { NORVM }\end{array}$ & $\begin{array}{l}\text { I. wreath/-- } \\
\text { //A SIS C. }\end{array}$ & $\begin{array}{l}\text { RIC 153, } \\
32 \mathrm{~b}\end{array}$ & G137-1 & \\
\hline 178 & $\begin{array}{l}\text { Theodosius } \\
\text { I-Honorius }\end{array}$ & $383-403$ & ÆE 4 & E & 12 & 0.72 & 10 & ? & $\begin{array}{l}\text { [SALVS R]EI- } \\
\text { PVBLI[CAE] }\end{array}$ & $?$ & incerta & G133-38 & \\
\hline 179 & Arcadius & $383-403$ & $\mathbb{E}_{4}$ & $\mathbb{E}$ & 12 & 0.81 & 12 & $\begin{array}{l}\text { DN ARCADI- } \\
\text { VS PF AVG }\end{array}$ & $\begin{array}{l}\text { [SALVS REI]- } \\
\text { PV[BLICAE] }\end{array}$ & $?$ & incerta & G133-100 & \\
\hline 180 & Theodosius I & $384 ?-387$ & Æ3 & $\mathbb{E}$ & 18 & 2.08 & 1 & $\begin{array}{l}\text { DN THEODO- } \\
\text { SIVS PF AVG }\end{array}$ & $\begin{array}{c}\text { GLORIA RO-MA- } \\
\text { NORVM }\end{array}$ & B SIS C. & $\begin{array}{l}\text { RIC 154, } \\
38 \mathrm{~b}\end{array}$ & G133-68 & \\
\hline 181 & Theodosius I & $384 ?-387$ & $\mathbb{E}_{4}$ & E & 14 & 1.14 & 12 & $\begin{array}{l}\text { DN THEODO- } \\
\text { SIVS PF AVG }\end{array}$ & $\begin{array}{l}\text { VICTOR-IA } \\
\text { AVGGG }\end{array}$ & A SIS C. & $\begin{array}{l}\text { RIC 155, } \\
39 \mathrm{~b}\end{array}$ & G126-15 & \\
\hline 182 & Arcadius & $388-393$ & $Æ_{4}$ & $\mathbb{E}$ & 13 & 1.07 & 6 & $\begin{array}{c}\text { [DN AR] } \\
\text { CADIVS [PF] } \\
\text { AVG }\end{array}$ & $\begin{array}{c}\text { SALVS REI-PVBLI- } \\
\text { CAE }\end{array}$ & $\mathrm{P} /--/ /$ TES $\Gamma$ & $\begin{array}{l}\text { RIC 188, } \\
65 \mathrm{C}\end{array}$ & G135-5 & \\
\hline
\end{tabular}




\begin{tabular}{|c|c|c|c|c|c|c|c|c|c|c|c|c|c|}
\hline \multicolumn{14}{|c|}{ SOTIN } \\
\hline $\begin{array}{l}\text { CAT. } \\
\text { NO. }\end{array}$ & RULER & $\begin{array}{l}\text { DATING } \\
\text { (YRS) }\end{array}$ & DENOM. & MAT. & $\begin{array}{c}\text { D. } \\
(\mathrm{mm})\end{array}$ & $\begin{array}{l}\text { WT. } \\
\text { (g) }\end{array}$ & $\begin{array}{l}\text { ORI- } \\
\text { ENT. }\end{array}$ & OBVERSE & REVERSE & $\begin{array}{l}\text { MINT } \\
\text { MARKS }\end{array}$ & $\begin{array}{l}\text { REFE- } \\
\text { RENCE }\end{array}$ & $\begin{array}{l}\text { INV. } \\
\text { NO. }\end{array}$ & NOTE \\
\hline 183 & Valentinian I & 364 & Æ 3 & $Æ$ & 20 & 2.3 & 7 & $\begin{array}{l}\text { DN VALENTI- } \\
\text { NI-ANVS PF } \\
\text { AVG }\end{array}$ & $\begin{array}{l}\text { RESTITV-TOR } \\
\text { REIP }\end{array}$ & B SIRM & $\begin{array}{c}\text { RIC 159, } \\
6 \mathrm{a}\end{array}$ & C23026 & NOTE \\
\hline 184 & Valentinian I & 364 & Æ 3 & $Æ$ & 18 & 2.04 & 1 & $\begin{array}{l}\text { DN VALENTI- } \\
\text { NI-ANVS PF } \\
\text { AVG }\end{array}$ & $\begin{array}{l}\text { SECVRITAS } \\
\text { REIPVBLICAE }\end{array}$ & B SIRM & $\begin{array}{l}\text { RIC 159, } \\
\quad 7 \mathrm{a}\end{array}$ & G60-236 & \\
\hline 185 & Valentinian I & 364 & E 3 & $Æ$ & 20 & 2.56 & 5 & $\begin{array}{c}\text { DN VALENTI- } \\
\text { NI-ANVS P[F } \\
\text { AVG] }\end{array}$ & VOT|V|MVLT|X & A SIRM & RIC 159,8 & C23413 & \\
\hline 186 & Valentinian I & 364 & Æ 3 & 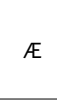 & 18 & 2.91 & 11 & $\begin{array}{l}\text { DN VALENTI- } \\
\text { NI-ANVS P[F } \\
\text { AVG] }\end{array}$ & VOT|V|MVLT|X & B SI[RM] & RIC 159,8 & G60-267 & \\
\hline 187 & Valens & 364 & E 3 & $\notin$ & 18 & 2.36 & 12 & $\begin{array}{c}\text { [DN VALEN]-S } \\
\text { PF AVG }\end{array}$ & $\begin{array}{l}\text { RESTITV-TOR } \\
\text { REIP }\end{array}$ & B SIRM & $\begin{array}{c}\text { RIC 159, } \\
6 \mathrm{~b}\end{array}$ & C23734 & \\
\hline 188 & Valentinian I & $364-367$ & $\mathbb{E}_{3}$ & $Æ$ & 18 & 2.02 & 1 & $\begin{array}{l}\text { DN VALENTI- } \\
\text { NI-[ANVS PF } \\
\text { AVG] }\end{array}$ & $\begin{array}{l}\text { [GLO]RIA RO- } \\
\text { MANORVM }\end{array}$ & $\begin{array}{c}--/ \mathrm{A}-/ /[\mathrm{SM}] \\
\mathrm{AQS}\end{array}$ & RIC 95, 7a & G60-258 & \\
\hline 189 & Valentinian I & $364-367$ & E 3 & $E$ & 18 & 2.51 & 5 & $\begin{array}{l}\text { DN VALENTI- } \\
\text { NI-ANVS PF } \\
\text { AVG }\end{array}$ & $\begin{array}{l}\text { GLORI[A RO- } \\
\text { MANORVM] }\end{array}$ & $\begin{array}{c}--/ A / / S M \\
A Q S\end{array}$ & RIC $95,7 \mathrm{a}$ & G61-42 & \\
\hline 190 & Valentinian I & $364-367$ & $\mathbb{E}_{3}$ & $Æ$ & 18 & 2.05 & 12 & $\begin{array}{c}\text { [DN V] } \\
\text { ALEN[TINI- } \\
\text { ANVS] PF } \\
\text { AVG }\end{array}$ & $\begin{array}{l}\text { GLORIA RO- } \\
\text { [MANORVM] }\end{array}$ & $\begin{array}{c}--/ A / / S M \\
A Q S\end{array}$ & RIC $95,7 \mathrm{a}$ & G61-62 & \\
\hline 191 & Valentinian I & $364-367$ & E 3 & $Æ$ & 20 & 2.27 & 6 & $\begin{array}{c}\text { DN VALENTI- } \\
\text { NI-[ANVS PF } \\
\text { AVG] }\end{array}$ & $\begin{array}{l}\text { GLORIA R[O- } \\
\text { MAN]ORVM }\end{array}$ & » $\Gamma$ SIS C & $\begin{array}{l}\text { RIC 146, } \\
5 a\end{array}$ & C22767 & \\
\hline 192 & Valentinian I & $364-367$ & Æ 3 & $Æ$ & 18 & 2.21 & 6 & $\begin{array}{c}\text { DN VALENTI- } \\
\text { NI-ANVS PF } \\
\text { AVG }\end{array}$ & $\begin{array}{l}\text { [GLORIA RO]- } \\
\text { MANORVM }\end{array}$ & D I SIS C & $\begin{array}{l}\text { RIC 146, } \\
5 a\end{array}$ & C22775 & \\
\hline 193 & Valentinian I & $364-367$ & Æ 3 & $Æ$ & 18 & 1.99 & 7 & $\begin{array}{c}\text { [DN VAL] } \\
\text { ENTINI-ANVS } \\
\text { PF [AVG] }\end{array}$ & $\begin{array}{l}\text { GLORIA RO-MA- } \\
\text { NORVM }\end{array}$ & $\begin{array}{c}--/ * \mathrm{~A} / / \mathrm{D} \Gamma \\
\mathrm{SISC}\end{array}$ & $\begin{array}{l}\text { RIC 146, } \\
5 a\end{array}$ & C22790 & \\
\hline 194 & Valentinian I & $364-367$ & $Æ_{3} 3$ & $Æ$ & 19 & 2.54 & 7 & $\begin{array}{l}\text { DN VALENTI- } \\
\text { NI-ANVS PF } \\
\text { AVG }\end{array}$ & $\begin{array}{l}\text { GLORIA RO-MA- } \\
\text { NORVM }\end{array}$ & $\begin{array}{c}--/ * \mathrm{~A} / / \mathrm{D} \Gamma \\
\mathrm{SISC}\end{array}$ & $\begin{array}{l}\text { RIC 146, } \\
5 a\end{array}$ & C22791 & \\
\hline 195 & Valentinian I & $364-367$ & $Æ_{3} 3$ & $Æ$ & 19 & 2.3 & 11 & $\begin{array}{c}\text { DN } \\
\text { VAL[ENTINI- } \\
\text { ANVS PF } \\
\text { AVG] }\end{array}$ & $\begin{array}{l}\text { GLORIA [RO]- } \\
\text { MANORVM }\end{array}$ & $\begin{array}{c}--/ * \mathrm{~A} / / \mathrm{D} \Gamma \\
\mathrm{SISC}\end{array}$ & $\begin{array}{l}\text { RIC 146, } \\
5 a\end{array}$ & G60-136 & \\
\hline 196 & Valentinian I & $364-367$ & $Æ_{3}$ & E & 17 & 1.93 & 5 & $\begin{array}{c}\text { [DN } \\
\text { VALENTINI]- } \\
\text { ANVS PF AVG }\end{array}$ & $\begin{array}{l}\text { [GLORIA] RO- } \\
\text { MANORVM }\end{array}$ & $D \Gamma S I S C$ & $\begin{array}{l}\text { RIC 146, } \\
5 a\end{array}$ & G60-138 & \\
\hline 197 & Valentinian I & $364-367$ & Æ 3 & $Æ$ & 18 & 1.97 & 7 & $\begin{array}{l}\text { DN VALENTI- } \\
\text { NI-ANVS PF } \\
\text { AVG }\end{array}$ & $\begin{array}{l}\text { [GL]ORIA RO- } \\
\text { MANORVM }\end{array}$ & $\begin{array}{c}--/ * \mathrm{~A} / / ? \Gamma \Gamma \\
\mathrm{SISC}\end{array}$ & $\begin{array}{l}\text { RIC 146, } \\
5 a\end{array}$ & G60-175 & \\
\hline 198 & Valentinian I & $364-367$ & E 3 & $\notin$ & 17 & 1.89 & 7 & $\begin{array}{l}\text { DN VALENTI- } \\
\text { NI-ANVS PF } \\
\text { AVG }\end{array}$ & $\begin{array}{l}\text { [GLORIA RO]- } \\
\text { MANO[RVM] }\end{array}$ & ? SIS C & $\begin{array}{l}\text { RIC 146, } \\
5 a\end{array}$ & G60-176 & \\
\hline 199 & Valentinian I & $364-367$ & $Æ_{3} 3$ & E & 18 & 1.4 & 12 & $\begin{array}{c}\text { [DN VALENT] } \\
\text { INI-ANV[S PF } \\
\text { AVG] }\end{array}$ & $\begin{array}{l}\text { [GLORI]A RO- } \\
\text { [MANORVM] }\end{array}$ & ? SIS C & $\begin{array}{l}\text { RIC 146, } \\
5 a\end{array}$ & G60-210 & \\
\hline 200 & Valentinian I & $364-367$ & $Æ_{3}$ & $Æ$ & 17 & 1.96 & 11 & $\begin{array}{c}\text { DN } \\
\text { VALE[NTINI]- } \\
\text { ANVS PF AVG }\end{array}$ & $\begin{array}{l}\text { GLORI[A] RO- } \\
\text { [MANORVM] }\end{array}$ & $\begin{array}{c}--/ * \mathrm{~A} / / \text { ? } \\
\mathrm{SISC}\end{array}$ & $\begin{array}{l}\text { RIC 146, } \\
5 a\end{array}$ & G60-213 & \\
\hline 201 & Valentinian I & $364-367$ & $Æ_{3} 3$ & $Æ$ & 19 & 2.62 & 7 & $\begin{array}{c}\text { [DN } \\
\text { VALENTINI]- } \\
\text { ANVS PF AVG }\end{array}$ & $\begin{array}{l}\text { GLORIA RO-MA- } \\
\text { NORVM }\end{array}$ & ? SIS C & $\begin{array}{l}\text { RIC 146, } \\
5 a\end{array}$ & G60-233 & \\
\hline 202 & Valentinian I & $364-367$ & Æ 3 & $Æ$ & 18 & 2.02 & 12 & $\begin{array}{c}\text { [DN } \\
\text { VALENTINI]- } \\
\text { ANVS PF AVG }\end{array}$ & $\begin{array}{l}\text { GLORIA RO- } \\
\text { [MANORVM] }\end{array}$ & $\begin{array}{c}--/ * \mathrm{~A} / / \mathrm{D}[\mathrm{C}] \\
\mathrm{SISC}\end{array}$ & $\begin{array}{l}\text { RIC 146, } \\
5 a\end{array}$ & G60-237 & \\
\hline 203 & Valentinian I & $364-367$ & $Æ_{3} 3$ & $Æ$ & 19 & 2.35 & 8 & $\begin{array}{c}\text { DN VALENTI- } \\
\text { NI-ANVS PF } \\
\text { AVG }\end{array}$ & $\begin{array}{l}\text { GLORIA RO-MA- } \\
\text { NORVM }\end{array}$ & $» \Gamma S I S C$ & $\begin{array}{l}\text { RIC 146, } \\
5 \mathrm{a}\end{array}$ & G60-42 & \\
\hline 204 & Valentinian I & $364-367$ & Æ 3 & $Æ$ & 19 & 2.24 & 1 & $\begin{array}{l}\text { DN VALENTI- } \\
\text { NI-ANVS PF } \\
\text { AVG }\end{array}$ & $\begin{array}{l}\text { GLORIA RO-MA- } \\
\text { NORVM }\end{array}$ & $\begin{array}{c}--/ * \mathrm{~A} / / \mathrm{D} \Gamma \\
\mathrm{SISC}\end{array}$ & $\begin{array}{l}\text { RIC 146, } \\
5 \mathrm{a}\end{array}$ & G60-45 & \\
\hline
\end{tabular}




\begin{tabular}{|c|c|c|c|c|c|c|c|c|c|c|c|c|c|}
\hline \multicolumn{14}{|c|}{ SOTIN } \\
\hline $\begin{array}{l}\text { CAT. } \\
\text { NO. }\end{array}$ & RULER & $\begin{array}{c}\text { DATING } \\
\text { (YRS) }\end{array}$ & DENOM. & MAT. & $\begin{array}{c}\mathrm{D} . \\
(\mathrm{mm})\end{array}$ & $\begin{array}{l}\text { WT. } \\
\text { (g) }\end{array}$ & $\begin{array}{l}\text { ORI- } \\
\text { ENT. }\end{array}$ & OBVERSE & REVERSE & $\begin{array}{c}\text { MINT } \\
\text { MARKS }\end{array}$ & $\begin{array}{l}\text { REFE- } \\
\text { RENCE }\end{array}$ & $\begin{array}{l}\text { INV. } \\
\text { NO. }\end{array}$ & NOTE \\
\hline 205 & Valentinian I & $364-367$ & A 3 & $Æ$ & 18 & 1.77 & 1 & $\begin{array}{c}\text { [DN } \\
\text { VALENTINI]- } \\
\text { ANVS PF AVG }\end{array}$ & $\begin{array}{l}\text { GLORIA RO-MA- } \\
\text { NORVM }\end{array}$ & $\begin{array}{c}--/ * \mathrm{~A} / / \mathrm{D} \Gamma \\
\mathrm{SISC}\end{array}$ & $\begin{array}{c}\text { RIC 146, } \\
5 a\end{array}$ & G60-46 & \\
\hline 206 & Valentinian I & $364-367$ & A 3 & $Æ$ & 19 & 2.58 & 12 & $\begin{array}{c}\text { DN VALENTI- } \\
\text { NI-ANVS PF } \\
\text { AVG }\end{array}$ & $\begin{array}{l}\text { GLORIA RO-MA- } \\
\text { NORVM }\end{array}$ & $\begin{array}{c}--/ * \mathrm{~A} / / \mathrm{D} \Gamma \\
\mathrm{SISC}\end{array}$ & $\begin{array}{c}\text { RIC 146, } \\
5 a\end{array}$ & G60-59 & \\
\hline 207 & Valentinian I & $364-367$ & A 3 & $Æ$ & 19 & 2.52 & 12 & $\begin{array}{c}\text { DN VALENTI- } \\
\text { NI-ANVS PF } \\
\text { AVG }\end{array}$ & $\begin{array}{l}\text { GLORIA RO-MA- } \\
\text { NORVM }\end{array}$ & $\cdot \Gamma \mathrm{SISC}$ & $\begin{array}{l}\text { RIC 146, } \\
5 a\end{array}$ & G6o-60 & \\
\hline 208 & Valentinian I & $364-367$ & E 3 & $Æ$ & 19 & 2.52 & 2 & $\begin{array}{c}\text { DN VALENTI- } \\
\text { NI-ANVS PF } \\
\text { [AVG] }\end{array}$ & $\begin{array}{l}\text { GLORIA RO-MA- } \\
\text { NORVM }\end{array}$ & $\begin{array}{c}--/ * \mathrm{~A} / / \cdot \Gamma \\
\text { SIS C }\end{array}$ & $\begin{array}{c}\text { RIC 146, } \\
5 \mathrm{a}\end{array}$ & G60-83 & \\
\hline 209 & Valentinian I & $364-367$ & A 3 & $Æ$ & 18 & 2.13 & 6 & $\begin{array}{c}\text { [DN } \\
\text { VALENTINI]- } \\
\text { ANVS PF AVG }\end{array}$ & $\begin{array}{l}\text { [GLORIA R]O- } \\
\text { MANORVM }\end{array}$ & » 「SIS C & $\begin{array}{c}\text { RIC 146, } \\
5 a\end{array}$ & G60-89 & \\
\hline 210 & Valentinian I & $364-367$ & Æ 3 & $Æ$ & 19 & 2.15 & 1 & $\begin{array}{c}\text { DN VALENTI- } \\
\text { NI-ANVS PF } \\
\text { AVG }\end{array}$ & $\begin{array}{l}\text { GLORIA RO-MA- } \\
\text { NORVM }\end{array}$ & $\cdot\ulcorner$ SIS C & $\begin{array}{l}\text { RIC 146, } \\
5 a\end{array}$ & G60-93 & \\
\hline 211 & Valentinian I & $364-367$ & A 3 & $Æ$ & 20 & 2.41 & 6 & $\begin{array}{c}\text { DN VALENTI- } \\
\text { NI-ANVS PF } \\
\text { AVG }\end{array}$ & $\begin{array}{l}\text { GLORIA RO-MA- } \\
\text { NORVM }\end{array}$ & » ГSIS C & $\begin{array}{l}\text { RIC 146, } \\
5 \mathrm{a}\end{array}$ & G60-99 & \\
\hline 212 & Valentinian I & $364-367$ & A 3 & $Æ$ & 17 & 2.27 & 12 & $\begin{array}{c}\text { DN VALENTI- } \\
\text { NI-ANVS PF } \\
\text { AVG }\end{array}$ & $\begin{array}{l}\text { GLORIA RO-MA- } \\
\text { NORVM }\end{array}$ & $\mathrm{D}[\Gamma] \mathrm{SIS} \mathrm{C}$ & $\begin{array}{l}\text { RIC 146, } \\
5 \mathrm{a}\end{array}$ & G61-27 & \\
\hline 213 & Valentinian I & $364-367$ & A 3 & $Æ$ & 18 & 2.09 & 12 & $\begin{array}{c}\text { DN VALENTI- } \\
\text { NI-ANVS PF } \\
\text { AVG }\end{array}$ & $\begin{array}{l}\text { GLORIA RO- } \\
\text { [MANORVM] }\end{array}$ & ? SIS C & $\begin{array}{c}\text { RIC 146, } \\
5 \mathrm{a}\end{array}$ & G61-28 & \\
\hline 214 & Valentinian I & $364-367$ & Æ 3 & $Æ$ & 17 & 1.98 & 7 & $\begin{array}{c}\text { [DN VALE] } \\
\text { NTINI-[ANVS } \\
\text { PF AVG] }\end{array}$ & $\begin{array}{l}\text { [GLORI]A RO- } \\
\text { [MANORVM] }\end{array}$ & $\begin{array}{l}--/ * \mathrm{~A} / / \cdot \Gamma \\
\text { SIS C }\end{array}$ & $\begin{array}{c}\text { RIC 146, } \\
5 \mathrm{a}\end{array}$ & G61-35 & \\
\hline 215 & Valentinian I & $364-367$ & Æ 3 & $Æ E$ & 18 & 2.07 & 12 & $\begin{array}{c}\text { DN VALENTI- } \\
\text { NI-ANVS PF } \\
\text { AVG }\end{array}$ & $\begin{array}{l}\text { GLORIA RO-MA- } \\
\text { NORVM }\end{array}$ & $\cdot \Gamma$ SIS C & $\begin{array}{l}\text { RIC 146, } \\
5 \mathrm{a}\end{array}$ & G61-7 & \\
\hline 216 & Valentinian I & $364-367$ & A 3 & $Æ$ & 18 & 2.32 & 12 & $\begin{array}{c}\text { DN VALENTI- } \\
\text { NI-ANVS PF } \\
\text { AVG }\end{array}$ & $\begin{array}{l}\text { GLORIA RO-MA- } \\
\text { NORVM }\end{array}$ & A SIS C & $\begin{array}{c}\text { RIC 146, } \\
5 \mathrm{a}\end{array}$ & G67-4 & \\
\hline 217 & Valentinian I & $364-367$ & Æ 3 & $Æ$ & 17 & 2.51 & 12 & $\begin{array}{c}{[\mathrm{DN}]} \\
\text { VALEN[TINI]- } \\
\text { ANVS PF AVG }\end{array}$ & $\begin{array}{l}\text { GLORIA RO- } \\
\text { [MANOR]VM }\end{array}$ & $--/ * / /$ TES ? & $\begin{array}{l}\text { RIC } 176 \\
16 \mathrm{a}\end{array}$ & G61-25 & \\
\hline 218 & Valentinian I & $364-367$ & Æ 3 & $Æ$ & 18 & 2.61 & 11 & $\begin{array}{c}\text { DN VALENTI- } \\
\text { NI-[ANVS PF] } \\
\text { AVG }\end{array}$ & $\begin{array}{c}\text { [GLORIA RO-MA] } \\
\text { NORVM }\end{array}$ & $\begin{array}{c}--/ * / / \\
\text { cons } \Delta\end{array}$ & $\begin{array}{l}\text { RIC 214, } \\
16 \mathrm{a}\end{array}$ & G60-165 & \\
\hline 219 & Valentinian I & $364-367$ & Æ 3 & $Æ$ & 18 & 1.87 & 6 & $\begin{array}{c}\text { D[N VALEN] } \\
\text { TINI-ANV[S } \\
\text { PF AVG] }\end{array}$ & $\begin{array}{c}\text { SECVRI[TAS } \\
\text { REIPVBLIC]AE }\end{array}$ & $\begin{array}{c}A /--/ / S M \\
\text { AQS }\end{array}$ & RIC 95, 9a & G60-48 & \\
\hline 220 & Valentinian I & $364-367$ & Æ 3 & $Æ$ & 20 & 1.9 & 6 & $\begin{array}{c}\text { DN VALENTI- } \\
\text { NI-ANVS PF } \\
\text { AVG }\end{array}$ & $\begin{array}{c}\text { SECVRITAS [REIP] } \\
\text { VBLICAE }\end{array}$ & * SM AQ P & RIC 95, 9a & G60-181 & \\
\hline 221 & Valentinian I & $364-367$ & Æ 3 & ÆE & 19 & 2.03 & 7 & $\begin{array}{c}\text { [DN } \\
\text { VALENTINI]- } \\
\text { ANVS PF AVG }\end{array}$ & $\begin{array}{l}\text { SECVRITAS } \\
\text { REIPVBLICAE }\end{array}$ & $\begin{array}{c}{[\mathrm{B}] \cdot /--/ / \mathrm{SM}} \\
\mathrm{AQS}\end{array}$ & RIC 95, 9a & G60-270 & \\
\hline 222 & Valentinian I & $364-367$ & Æ 3 & $Æ E$ & 18 & 1.64 & 12 & $\begin{array}{c}\text { DN VALENTI- } \\
\text { NI-ANVS PF } \\
\text { AVG }\end{array}$ & $\begin{array}{l}\text { SECVRITAS } \\
\text { REIPVBLICAE }\end{array}$ & $\begin{array}{c}--/ A / / S M \\
\text { AQ S }\end{array}$ & RIC 95, 7a & G61-22 & \\
\hline 223 & Valentinian I & $364-367$ & Æ 3 & $Æ$ & 19 & 2.77 & 1 & $\begin{array}{c}\text { DN VALENTI- } \\
\text { NI-ANVS PF } \\
\text { AVG }\end{array}$ & $\begin{array}{l}\text { SECVRITAS } \\
\text { REIPVBLICAE }\end{array}$ & $\cdot \Delta$ SIS C & $\begin{array}{c}\text { RIC 146, } \\
7 a\end{array}$ & C23165 & \\
\hline 224 & Valentinian I & $364-367$ & Æ 3 & $Æ$ & 18 & 2.16 & 1 & $\begin{array}{c}\text { DN VALENTI- } \\
\text { NI-ANVS PF } \\
\text { AVG }\end{array}$ & $\begin{array}{l}\text { SECVRITAS } \\
\text { REIPVBLICAE }\end{array}$ & $\begin{array}{l}* \mathrm{~A} /--/ / \mathrm{D} \Delta \\
\mathrm{SISC}\end{array}$ & $\begin{array}{c}\text { RIC 146, } \\
7 a\end{array}$ & C23203 & \\
\hline 225 & Valentinian I & $364-367$ & Æ 3 & $Æ$ & 18 & 2.11 & 1 & $\begin{array}{c}\text { DN VALENTI- } \\
\text { NI-ANVS PF } \\
\text { AVG }\end{array}$ & $\begin{array}{l}\text { SECVRITAS } \\
\text { REIPVBLICAE }\end{array}$ & A SIS C & $\begin{array}{l}\text { RIC 146, } \\
7 a\end{array}$ & C25783 & \\
\hline 226 & Valentinian I & $364-367$ & Æ 3 & $Æ$ & 18 & 2.57 & 6 & $\begin{array}{c}\text { DN VALENTI- } \\
\text { NI-ANVS PF } \\
\text { AVG }\end{array}$ & $\begin{array}{l}\text { SECVRITAS } \\
\text { REIPVBLICAE }\end{array}$ & B SIS C & $\begin{array}{c}\text { RIC 146, } \\
7 \mathrm{a}\end{array}$ & G60-20 & \\
\hline
\end{tabular}




\begin{tabular}{|c|c|c|c|c|c|c|c|c|c|c|c|c|c|}
\hline \multicolumn{14}{|c|}{ SOTIN } \\
\hline $\begin{array}{l}\text { CAT. } \\
\text { NO. }\end{array}$ & RULER & $\begin{array}{l}\text { DATING } \\
\text { (YRS) }\end{array}$ & DENOM. & MAT. & $\begin{array}{c}\text { D. } \\
(\mathrm{mm})\end{array}$ & $\begin{array}{l}\text { WT. } \\
\text { (g) }\end{array}$ & $\begin{array}{l}\text { ORI- } \\
\text { ENT. }\end{array}$ & OBVERSE & REVERSE & $\begin{array}{c}\text { MINT } \\
\text { MARKS }\end{array}$ & $\begin{array}{c}\text { REFE- } \\
\text { RENCE }\end{array}$ & $\begin{array}{l}\text { INV. } \\
\text { NO. }\end{array}$ & NOTE \\
\hline 227 & Valentinian I & $364-367$ & Æ 3 & $\mathscr{E}$ & 19 & 2.33 & 7 & $\begin{array}{l}\text { DN VALENTI- } \\
\text { NI-ANVS PF } \\
\text { AVG }\end{array}$ & $\begin{array}{l}\text { SECVRITAS } \\
\text { REIPVBLICAE }\end{array}$ & $» \Delta S I S C$ & $\begin{array}{c}\text { RIC 146, } \\
7 a\end{array}$ & G60-30 & \\
\hline 228 & Valentinian I & $364-367$ & Æ 3 & $\mathscr{F}$ & 19 & 2.01 & 6 & $\begin{array}{l}\text { DN VALENTI- } \\
\text { NI-ANVS PF } \\
\text { AVG }\end{array}$ & $\begin{array}{l}\text { SECVRITAS } \\
\text { REIPVBLICAE }\end{array}$ & $\mathrm{D} \Delta \mathrm{SISC}$ & $\begin{array}{c}\text { RIC 146, } \\
7 a\end{array}$ & G60-52 & \\
\hline 229 & Valentinian I & $364-367$ & E 3 & $F$ & 19 & 2.14 & 12 & $\begin{array}{l}\text { DN VALENTI- } \\
\text { NI-ANVS PF } \\
\text { AVG }\end{array}$ & $\begin{array}{c}\text { SECVRITAS } \\
\text { [REIPVBLICAE] }\end{array}$ & $\cdot \Delta \operatorname{SISC}$ & $\begin{array}{c}\text { RIC 146, } \\
7 a\end{array}$ & G60-69 & \\
\hline 230 & Valentinian I & $364-367$ & Æ 3 & F & 19 & 2.31 & 7 & $\begin{array}{l}\text { DN VALENTI- } \\
\text { NI-ANVS PF } \\
\text { AVG }\end{array}$ & $\begin{array}{l}\text { SECVRIT[AS] } \\
\text { REIPVBLICAE }\end{array}$ & $" \Delta \mathrm{SISC}$ & $\begin{array}{c}\text { RIC 146, } \\
7 a\end{array}$ & G60-74 & \\
\hline 231 & Valentinian I & $364-367$ & E 3 & F & 18 & 2.15 & 6 & $\begin{array}{l}\text { DN VALENTI- } \\
\text { NI-ANVS PF } \\
\text { AVG }\end{array}$ & $\begin{array}{l}\text { SECVRITAS } \\
\text { REIPVBLICAE }\end{array}$ & $\begin{array}{c}* \mathrm{~A} /--/ / \mathrm{D} \Delta \\
\mathrm{SISC}\end{array}$ & $\begin{array}{c}\text { RIC 146, } \\
7 a\end{array}$ & G60-95 & \\
\hline 232 & Valentinian I & $364-367$ & Æ 3 & F & 18 & 2.59 & 6 & $\begin{array}{l}\text { DN VALENTI- } \\
\text { NI-[ANVS PF } \\
\text { AVG] }\end{array}$ & $\begin{array}{l}\text { SECVRITAS } \\
\text { REIPVBLICAE }\end{array}$ & - A SIS C & $\begin{array}{c}\text { RIC } 146, \\
7 a\end{array}$ & G60-143 & \\
\hline 233 & Valentinian I & $364-367$ & Æ 3 & $F$ & 19 & 2.91 & 12 & $\begin{array}{l}\text { DN VALENTI- } \\
\text { NI-ANVS PF } \\
\text { AVG }\end{array}$ & $\begin{array}{l}\text { SECVRITAS } \\
\text { REIPVBLICAE }\end{array}$ & $\begin{array}{c}* \mathrm{~A} /--/ / \mathrm{D} \mathrm{A} \\
\mathrm{SISC}\end{array}$ & $\begin{array}{c}\text { RIC 146, } \\
7 a\end{array}$ & G60-150 & \\
\hline 234 & Valentinian I & $364-367$ & Æ 3 & FE & 19 & 2.01 & 7 & $\begin{array}{l}\text { DN VALENTI- } \\
\text { NI-ANVS PF } \\
\text { AVG] }\end{array}$ & $\begin{array}{l}\text { [SECVRITAS } \\
\text { REIPVBLI]CAE }\end{array}$ & $\cdot \Delta \operatorname{SISC}$ & $\begin{array}{l}\text { RIC } 146 \\
7 a\end{array}$ & G60-166 & \\
\hline 235 & Valentinian I & $364-367$ & Æ 3 & $F$ & 20 & 2.62 & 7 & $\begin{array}{l}\text { DN VALENTI- } \\
\text { NI-ANVS PF } \\
\text { AVG }\end{array}$ & $\begin{array}{c}\text { SECVRITAS } \\
\text { [REIPVBLICAE] }\end{array}$ & $\Delta \mathrm{SISC}$ & $\begin{array}{c}\text { RIC } 146, \\
7 a\end{array}$ & G60-172 & \\
\hline 236 & Valentinian I & $364-367$ & Æ3 & FE & 20 & 2.37 & 1 & $\begin{array}{c}\text { DN } \\
\text { VALENTIN[I- } \\
\text { ANVS PF } \\
\text { AVG] }\end{array}$ & $\begin{array}{l}\text { [SECVRITAS] } \\
\text { REIPVBLICAE }\end{array}$ & $\mathrm{D} \Delta \mathrm{SISC}$ & $\begin{array}{c}\text { RIC 146, } \\
7 a\end{array}$ & G60-195 & \\
\hline 237 & Valentinian I & $364-367$ & Æ 3 & FE & 19 & 1.39 & 1 & $\begin{array}{l}\text { DN VALENTI- } \\
\text { NI-ANVS [PF } \\
\text { AVG] }\end{array}$ & $\begin{array}{l}\text { SECVRITAS } \\
\text { REIPVBLICAE }\end{array}$ & - A SIS C & $\begin{array}{c}\text { RIC 146, } \\
7 a\end{array}$ & G60-199 & \\
\hline 238 & Valentinian I & $364-367$ & $Æ_{3} 3$ & E & 19 & 2.29 & 6 & $\begin{array}{c}\text { DN } \\
\text { VALE[NTINI- } \\
\text { ANVS] PF } \\
\text { AVG }\end{array}$ & $\begin{array}{l}\text { SECVRITAS } \\
\text { REIPVBLICAE }\end{array}$ & $\mathrm{D} \Delta \mathrm{SISC}$ & $\begin{array}{c}\text { RIC 146, } \\
7 a\end{array}$ & G60-265 & \\
\hline 239 & Valentinian I & $364-367$ & $Æ_{3}$ & $\notin$ & 19 & 2.46 & 7 & $\begin{array}{c}\text { DN VALENTI- } \\
\text { NI-ANVS PF } \\
\text { AVG }\end{array}$ & $\begin{array}{l}\text { SECVRITAS } \\
\text { REIPVBLICAE }\end{array}$ & $\begin{array}{c}* \mathrm{~A} /--/ / \mathrm{D} \mathrm{A} \\
\text { SIS C }\end{array}$ & $\begin{array}{c}\text { RIC } 146 \\
7 a\end{array}$ & G61-2 & \\
\hline 240 & Valentinian I & $364-367$ & Æ 3 & $\notin$ & 18 & 2.34 & 1 & $\begin{array}{c}\text { [DN } \\
\text { VALENTINI]- } \\
\text { ANVS PF AVG }\end{array}$ & $\begin{array}{c}\text { SECVRITAS } \\
\text { REIPVBLI[CAE] }\end{array}$ & $\mathrm{D} \Delta \mathrm{SISC}$ & $\begin{array}{c}\text { RIC 146, } \\
7 a\end{array}$ & G61-30 & \\
\hline 241 & Valentinian I & $364-367$ & Æ 3 & EE & 19 & 2.22 & 1 & $\begin{array}{l}\text { DN VALENTI- } \\
\text { NI-ANVS PF } \\
\text { AVG }\end{array}$ & $\begin{array}{l}\text { SECVRITAS } \\
\text { REIPVBLICAE }\end{array}$ & $\mathrm{D} \Delta \mathrm{SISC}$ & $\begin{array}{c}\text { RIC 146, } \\
7 a\end{array}$ & G61-34 & \\
\hline 242 & Valentinian I & $364-367$ & $\star_{3} 3$ & E & 19 & 2.1 & 2 & $\begin{array}{c}\text { [DN V]ALEN- } \\
\text { TINI-[ANVS } \\
\text { PF AVG] }\end{array}$ & $\begin{array}{l}\text { SECVRITAS } \\
\text { REIPVBLICAE }\end{array}$ & $\begin{array}{l}* \mathrm{~A} /--/ / \mathrm{D} \\
\mathrm{ASISC}\end{array}$ & $\begin{array}{l}\text { RIC 146, } \\
7 a\end{array}$ & G61-59 & \\
\hline 243 & Valentinian I & $364-367$ & $\mathbb{E}_{3}$ & EE & 18 & 2.71 & 5 & $\begin{array}{l}\text { DN VALENTI- } \\
\text { NI-ANVS PF } \\
\text { AVG }\end{array}$ & $\begin{array}{l}\text { SECVRITAS } \\
\text { REIPVBLICAE }\end{array}$ & TES A & $\begin{array}{c}\text { RIC 176, } \\
18 a\end{array}$ & C23366 & \\
\hline 244 & Valentinian I & $364-367$ & $Æ_{3} 3$ & EE & 18 & 2.24 & 11 & $\begin{array}{l}\text { DN VALENTI- } \\
\text { NI-ANVS PF } \\
\text { AVG }\end{array}$ & $\begin{array}{c}\text { SECVRITAS } \\
\text { [REIPVBLICAE] }\end{array}$ & TES A & $\begin{array}{c}\text { RIC 176, } \\
18 \mathrm{a}\end{array}$ & G60-77 & \\
\hline 245 & Valentinian I & $364-367$ & Æ 3 & E & 18 & 2.14 & 10 & $\begin{array}{c}\text { [DN VALEN] } \\
\text { TINI-ANVS PF } \\
\text { AVG }\end{array}$ & $\begin{array}{c}\text { SECVRITAS } \\
\text { REIPV[BLICAE] }\end{array}$ & $\operatorname{TES}[A]$ & $\begin{array}{c}\text { RIC 176, } \\
18 a\end{array}$ & G60-157 & \\
\hline 246 & Valentinian I & $364-367$ & Æ3 & $\notin$ & 18 & 2.58 & 11 & $\begin{array}{c}\text { [DN } \\
\text { VALENTINI]- } \\
\text { ANVS [PF } \\
\text { AVG] }\end{array}$ & $\begin{array}{c}\text { SECVRITAS } \\
{[\text { REIPV]BLICAE }}\end{array}$ & TES A & $\begin{array}{c}\text { RIC 176, } \\
18 a\end{array}$ & G60-203 & \\
\hline 247 & Valentinian I & $364-367$ & E 3 & E & 18 & 2.13 & 5 & $\begin{array}{c}\text { DN VALENTI- } \\
\text { NI-ANVS P[F } \\
\text { AVG] }\end{array}$ & $\begin{array}{l}\text { [SECV]RITAS } \\
\text { REIPVBLICAE }\end{array}$ & $* /--/ /$ TES A & $\begin{array}{l}\text { RIC 176, } \\
18 a\end{array}$ & G60-206 & \\
\hline
\end{tabular}




\begin{tabular}{|c|c|c|c|c|c|c|c|c|c|c|c|c|c|}
\hline \multicolumn{14}{|c|}{ SOTIN } \\
\hline $\begin{array}{l}\text { CAT. } \\
\text { NO. }\end{array}$ & RULER & $\begin{array}{l}\text { DATING } \\
\text { (YRS) }\end{array}$ & DENOM. & MAT. & $\begin{array}{c}\mathrm{D} . \\
(\mathrm{mm})\end{array}$ & $\begin{array}{l}\text { WT. } \\
\text { (g) }\end{array}$ & $\begin{array}{l}\text { ORI- } \\
\text { ENT. }\end{array}$ & OBVERSE & REVERSE & $\begin{array}{l}\text { MINT } \\
\text { MARKS }\end{array}$ & $\begin{array}{l}\text { REFE- } \\
\text { RENCE }\end{array}$ & $\begin{array}{l}\text { INV. } \\
\text { NO. }\end{array}$ & NOTE \\
\hline 248 & Valentinian I & $364-367$ & $\mathbb{E}_{3}$ & $\mathbb{E}$ & 17 & 2.24 & 5 & $\begin{array}{l}\text { DN VALENTI- } \\
\text { NI-ANVS PF } \\
\text { AVG }\end{array}$ & $\begin{array}{l}\text { SECVRITAS } \\
\text { REIPVBLICAE }\end{array}$ & TES A & $\begin{array}{c}\text { RIC 176, } \\
18 \mathrm{a}\end{array}$ & G60-239 & \\
\hline 249 & Valentinian I & $364-367$ & solidus & AV & 22 & 4.42 & 1 & $\begin{array}{l}\text { DN VALENTI- } \\
\text { NI-ANVS PF } \\
\text { AVG }\end{array}$ & $\begin{array}{l}\text { RESTITVTOR } \\
\text { REIPUBLICAE }\end{array}$ & ANTS & $\begin{array}{l}\text { RIC 272, } \\
2 \mathrm{a}\end{array}$ & C31441 & \\
\hline 250 & Valentinian I & $364-367$ & Æ 3 & E & 18 & 1.91 & 7 & $\begin{array}{l}\text { DN VALENTI- } \\
\text { NI-ANVS PF } \\
\text { AVG }\end{array}$ & $\begin{array}{l}\text { RESTITV-TOR } \\
\text { REIPVB }\end{array}$ & TES B & $\begin{array}{l}\text { RIC 176, } \\
17 a\end{array}$ & G71-2 & \\
\hline 251 & Valentinian I & $364-367$ & E 3 & E & 19 & 2.55 & 6 & $\begin{array}{l}\text { DN VALENTI- } \\
\text { NI-ANVS PF } \\
\text { AVG }\end{array}$ & $\begin{array}{c}\text { RESTITV-[TOR } \\
\text { RE]IP }\end{array}$ & CONSP $\mathrm{P}$ & $\begin{array}{l}\text { RIC 215, } \\
\text { 20a }\end{array}$ & C23007 & \\
\hline 252 & Valens & $364-367$ & E 3 & $\mathbb{E}$ & 18 & 2.74 & 6 & $\begin{array}{l}\text { DN VALEN-S } \\
\text { PF AVG }\end{array}$ & $\begin{array}{l}\text { GLORIA RO-MA- } \\
\text { NORVM }\end{array}$ & $\begin{array}{c}--/ B \cdot / / S M \\
A Q S\end{array}$ & RIC 95, 7b & C23473 & \\
\hline 253 & Valens & $364-367$ & $Æ_{3}$ & $\mathbb{E}$ & 18 & 2.11 & 7 & $\begin{array}{l}\text { DN VALEN-S } \\
\text { PF AVG }\end{array}$ & $\begin{array}{l}\text { GLORIA RO-MA- } \\
\text { NORVM }\end{array}$ & * SM AQS & RIC 95, 7b & G60-189 & \\
\hline 254 & Valens & $364-367$ & $\mathbb{E}_{3}$ & $\mathbb{E}$ & 19 & 2.1 & 12 & $\begin{array}{l}\text { DN VALEN-[S } \\
\text { PF AVG ] }\end{array}$ & $\begin{array}{l}\text { [GLO]RIA RO- } \\
\text { MAN[O]RVM }\end{array}$ & $* \mathrm{SM} A Q \mathrm{~S}$ & RIC 95, 7b & G61-57 & \\
\hline 255 & Valens & $364-367$ & $\mathbb{E}_{3}$ & $\mathbb{E}$ & 17 & 2.23 & 7 & $\begin{array}{l}\text { DN VALEN-S } \\
\text { PF AVG }\end{array}$ & $\begin{array}{c}\text { GLORIA RO-MA- } \\
\text { NORVM }\end{array}$ & $\begin{array}{c}--/ * \mathrm{~A} / / \mathrm{DB} \\
\mathrm{SISC}\end{array}$ & $\begin{array}{l}\text { RIC } 146 \text {, } \\
5 \mathrm{~b}\end{array}$ & G60-35 & \\
\hline 256 & Valens & $364-367$ & E 3 & $\mathbb{E}$ & 18 & 2.18 & 6 & $\begin{array}{l}\text { DN VALEN-S } \\
\text { PF AVG }\end{array}$ & $\begin{array}{l}\text { GLORIA RO-MA- } \\
\text { NORVM }\end{array}$ & B SIS C & $\begin{array}{l}\text { RIC } 146 \text {, } \\
5 \mathrm{~b}\end{array}$ & G60-65 & \\
\hline 257 & Valens & $364-367$ & $Æ 3$ & $\mathbb{E}$ & 19 & 1.78 & 7 & $\begin{array}{l}\text { DN VALEN-S } \\
\text { PF AVG }\end{array}$ & $\begin{array}{l}\text { [GLORIA R]O- } \\
\text { MANORVM }\end{array}$ & $\begin{array}{c}--/ * \mathrm{~A} / / \cdot \mathrm{B} \\
\mathrm{SISC}\end{array}$ & $\begin{array}{l}\text { RIC } 146 \text {, } \\
5 \mathrm{~b}\end{array}$ & G60-94 & \\
\hline 258 & Valens & $364-367$ & Æ 3 & $\mathbb{E}$ & 18 & 1.72 & 2 & $\begin{array}{l}\text { DN VALEN-S } \\
\text { PF AVG }\end{array}$ & $\begin{array}{l}\text { GLORIA RO-MA- } \\
\text { NORVM }\end{array}$ & A SIS C & $\begin{array}{l}\text { RIC } 146 \text {, } \\
5 \mathrm{~b}\end{array}$ & G60-149 & \\
\hline 259 & Valens & $364-367$ & $Æ_{3}$ & $A E$ & 18 & 3.06 & 11 & $\begin{array}{l}\text { DN VALEN-S } \\
\text { PF AVG }\end{array}$ & $\begin{array}{l}\text { GLORIA RO-MA- } \\
\text { NORVM }\end{array}$ & D B SIS C & $\begin{array}{l}\text { RIC } 146 \text {, } \\
5 \mathrm{~b}\end{array}$ & G60-160 & \\
\hline 260 & Valens & $364-367$ & Æ 3 & $\mathbb{E}$ & 20 & 2.36 & 7 & $\begin{array}{l}\text { [DN V]ALEN-S } \\
\text { PF AVG }\end{array}$ & $\begin{array}{l}\text { [GLORIA] RO- } \\
\text { MANORVM }\end{array}$ & D B SIS C & $\begin{array}{l}\text { RIC } 146 \text {, } \\
5 \mathrm{~b}\end{array}$ & G60-170 & \\
\hline 261 & Valens & $364-367$ & $Æ_{3}$ & $\mathbb{E}$ & 18 & 1.98 & 7 & $\begin{array}{l}\text { DN VA[LEN-S } \\
\text { PF AVG ] }\end{array}$ & $\begin{array}{l}\text { GLORIA RO- } \\
\text { [MANORVM] }\end{array}$ & $\begin{array}{c}--/ * \mathrm{~A} / / \mathrm{DB} \\
\mathrm{SISC}\end{array}$ & $\begin{array}{l}\text { RIC } 146, \\
5 \mathrm{~b}\end{array}$ & G60-185 & \\
\hline 262 & Valens & $364-367$ & Æ 3 & $\mathbb{E}$ & 17 & 1.78 & 7 & $\begin{array}{c}\text { [DN VALEN]-S } \\
\text { PF [AVG] }\end{array}$ & $\begin{array}{l}\text { [GLORIA RO]- } \\
\text { MANORVM }\end{array}$ & $\begin{array}{c}--/ * \mathrm{~A} / / \cdot \mathrm{B} \\
\mathrm{SISC}\end{array}$ & $\begin{array}{l}\text { RIC } 146 \text {, } \\
5 \mathrm{~b}\end{array}$ & G60-289 & \\
\hline 263 & Valens & $364-367$ & $\mathbb{E}_{3}$ & E & 18 & 2.59 & 2 & $\begin{array}{l}\text { DN VALEN-S } \\
\text { PF AVG }\end{array}$ & $\begin{array}{c}\text { GLORIA RO-MA- } \\
\text { NORVM }\end{array}$ & $\begin{array}{c}--/ * \mathrm{~A} / / \mathrm{DB} \\
\mathrm{SISC}\end{array}$ & $\begin{array}{l}\text { RIC } 146, \\
5 b\end{array}$ & G61-13 & \\
\hline 264 & Valens & $364-367$ & $E_{3}$ & E & 18 & 2.2 & 1 & $\begin{array}{c}\text { [DN VALE] } \\
\mathrm{N}-[\mathrm{S} \text { PF AVG] }\end{array}$ & $\begin{array}{l}\text { [GLORIA RO-] } \\
\text { MAN[ORVM] }\end{array}$ & ? SIS C & $\begin{array}{l}\text { RIC } 146 \text {, } \\
5 \mathrm{~b}\end{array}$ & G61-37 & \\
\hline 265 & Valens & $364-367$ & $\mathbb{E}_{3}$ & E & 19 & 2.35 & 12 & $\begin{array}{l}\text { DN VALEN-S } \\
\text { PF AVG }\end{array}$ & $\begin{array}{l}\text { GL[OR]IA RO- } \\
\text { MANORVM }\end{array}$ & - B SIS C & $\begin{array}{l}\text { RIC } 146, \\
5 \mathrm{~b}\end{array}$ & G61-47 & \\
\hline 266 & Valens & $364-367$ & $Æ_{3}$ & E & 18 & 1.98 & 1 & $\begin{array}{l}\text { DN VALEN-S } \\
\text { PF AVG }\end{array}$ & $\begin{array}{l}\text { GLORIA RO-MA- } \\
\text { NORVM }\end{array}$ & - A SIS C & $\begin{array}{l}\text { RIC } 146 \text {, } \\
5 \mathrm{~b}\end{array}$ & G67-3 & \\
\hline 267 & Valens & $364-367$ & $Æ_{3}$ & E & 18 & 2.33 & 6 & $\begin{array}{l}\text { DN VALEN-S } \\
\text { PF AVG }\end{array}$ & $\begin{array}{c}\text { GLORIA RO-MA- } \\
\text { NORVM }\end{array}$ & - B SIS C & $\begin{array}{l}\text { RIC } 146 \text {, } \\
5 \mathrm{~b}\end{array}$ & G71-1 & \\
\hline 268 & Valens & $364-367$ & $Æ_{3}$ & $\mathbb{E}$ & 20 & 2.53 & 7 & $\begin{array}{l}\text { DN VALEN-S } \\
\text { PF AVG }\end{array}$ & $\begin{array}{c}\text { GLORIA RO-MA- } \\
\text { NORVM }\end{array}$ & - A SIS C & $\begin{array}{l}\text { RIC } 146 \text {, } \\
5 \mathrm{~b}\end{array}$ & C23529 & \\
\hline 269 & Valens & $364-367$ & $Æ_{3} 3$ & E & 19 & 2.34 & 12 & $\begin{array}{l}\text { DN VALEN-S } \\
\text { PF AVG }\end{array}$ & $\begin{array}{c}\text { GLORIA RO-MA- } \\
\text { NORVM }\end{array}$ & - B SIS C & $\begin{array}{l}\text { RIC } 146 \text {, } \\
5 \mathrm{~b}\end{array}$ & C23530 & \\
\hline 270 & Valens & $364-367$ & $Æ_{3} 3$ & $\mathbb{E}$ & 18 & 2.55 & 6 & $\begin{array}{l}\text { DN VALEN-S } \\
\text { PF AVG }\end{array}$ & $\begin{array}{c}\text { GLORIA RO-MA- } \\
\text { NORVM }\end{array}$ & - B SIS C & $\begin{array}{l}\text { RIC } 146 \text {, } \\
5 \mathrm{~b}\end{array}$ & C23531 & \\
\hline 271 & Valens & $364-367$ & $E_{3} 3$ & $\notin$ & 19 & 2.42 & 7 & $\begin{array}{l}\text { DN VALEN-S } \\
\text { PF AVG }\end{array}$ & $\begin{array}{l}\text { GLORIA RO-MA- } \\
\text { NORVM }\end{array}$ & B SIS C & $\begin{array}{l}\text { RIC } 146 \text {, } \\
5 \mathrm{~b}\end{array}$ & C23532 & \\
\hline 272 & Valens & $364-367$ & $E_{3}$ & $\notin$ & 18 & 2.35 & 1 & $\begin{array}{l}\text { DN VALEN-S } \\
\text { PF AVG }\end{array}$ & $\begin{array}{c}\text { GLORIA RO-MA- } \\
\text { NORVM }\end{array}$ & " B SIS C & $\begin{array}{l}\text { RIC } 146 \text {, } \\
5 \mathrm{~b}\end{array}$ & C23565 & \\
\hline 273 & Valens & $364-367$ & A 3 & $\notin$ & 18 & 2.22 & 6 & $\begin{array}{l}\text { DN VALEN-S } \\
\text { PF AVG }\end{array}$ & $\begin{array}{l}\text { GLORI[A RO]- } \\
\text { MANORVM }\end{array}$ & $\begin{array}{c}--/ * \mathrm{~A} / / \cdot \mathrm{B} \\
\mathrm{SISC}\end{array}$ & $\begin{array}{l}\text { RIC } 146, \\
5 b\end{array}$ & C23582 & \\
\hline 274 & Valens & $364-367$ & $E_{3}$ & $\notin$ & 18 & 2.19 & 5 & $\begin{array}{l}\text { DN VALEN-S } \\
\text { PF AVG }\end{array}$ & $\begin{array}{c}\text { GLORIA RO-MA- } \\
\text { NORVM }\end{array}$ & TES $\Gamma$ & $\begin{array}{l}\text { RIC 176, } \\
16 \mathrm{~b}\end{array}$ & G60-163 & \\
\hline 275 & Valens & $364-367$ & $\notin 3$ & $\notin$ & 17 & 2.53 & 11 & $\begin{array}{l}\text { DN VALEN-[S } \\
\text { PF AVG ] }\end{array}$ & $\begin{array}{l}\text { [GLORIA RO- } \\
\text { MANO]RVM }\end{array}$ & TES S & $\begin{array}{l}\text { RIC 176, } \\
16 \mathrm{~b}\end{array}$ & G60-205 & \\
\hline 276 & Valens & $364-367$ & $E_{3} 3$ & E & 17 & 2.53 & 6 & $\begin{array}{l}\text { DN VALEN-S } \\
\text { PF AVG }\end{array}$ & $\begin{array}{l}\text { GLORIA [RO]- } \\
\text { MANORVM }\end{array}$ & TES $\Gamma$ & $\begin{array}{l}\text { RIC 176, } \\
16 \mathrm{~b}\end{array}$ & G61-32 & \\
\hline
\end{tabular}




\begin{tabular}{|c|c|c|c|c|c|c|c|c|c|c|c|c|c|}
\hline \multicolumn{14}{|c|}{ SOTIN } \\
\hline $\begin{array}{l}\text { CAT. } \\
\text { NO. }\end{array}$ & RULER & $\begin{array}{l}\text { DATING } \\
\text { (YRS) }\end{array}$ & DENOM. & MAT. & $\begin{array}{c}\text { D. } \\
(\mathrm{mm})\end{array}$ & $\begin{array}{l}\text { WT. } \\
\text { (g) }\end{array}$ & $\begin{array}{l}\text { ORI- } \\
\text { ENT. }\end{array}$ & OBVERSE & REVERSE & $\begin{array}{l}\text { MINT } \\
\text { MARKS }\end{array}$ & $\begin{array}{l}\text { REFE- } \\
\text { RENCE }\end{array}$ & $\begin{array}{l}\text { INV. } \\
\text { NO. }\end{array}$ & NOTE \\
\hline 277 & Valens & $364-367$ & E 3 & $F$ & 17 & 1.96 & 12 & $\begin{array}{l}\text { DN VALEN-S } \\
\text { PF [AVG] }\end{array}$ & $\begin{array}{c}\text { [G]LORIA RO-[M] } \\
\text { ANORVM }\end{array}$ & SMN & $\begin{array}{c}\text { RIC 252, } \\
9 \mathrm{C}\end{array}$ & C23509 & \\
\hline 278 & Valens & $364-367$ & E 3 & E & 19 & 2.02 & 7 & $\begin{array}{l}\text { DN VALENS- } \\
\text { P[F AVG] }\end{array}$ & $\begin{array}{l}\text { GLORI[A RO- } \\
\text { MAN]ORVM }\end{array}$ & SM N B & $\begin{array}{c}\text { RIC 252, } \\
9 \mathrm{C}\end{array}$ & G60-164 & \\
\hline 279 & Valens & $364-367$ & E 3 & $\mathscr{E}$ & 18 & 1.81 & 11 & $\begin{array}{l}\text { DN VALEN-S } \\
\text { P[F AVG ] }\end{array}$ & $\begin{array}{c}\text { GLORIA RO-[MA] } \\
\text { NORVM }\end{array}$ & SM N A & $\begin{array}{c}\text { RIC } 252 \\
2 a\end{array}$ & G60-224 & \\
\hline 280 & Valens & $364-367$ & E 3 & E & 17 & 2.37 & 7 & $\begin{array}{l}\text { DN VALENS } \\
\text { PF A[VG] }\end{array}$ & $\begin{array}{l}\text { GLORIA [RO- } \\
\text { MANORVM] }\end{array}$ & $\mathrm{SM} N \Delta$ & $\begin{array}{c}\text { RIC 252, } \\
9 \mathrm{C}\end{array}$ & G60-235 & \\
\hline 281 & Valens & $364-367$ & E 3 & $F$ & 17 & 2.01 & 12 & $\begin{array}{c}\text { DN VALEN-[S } \\
\text { PF AVG] }\end{array}$ & $\begin{array}{l}\text { [SECVRITAS] } \\
\text { REIPVBLICAE }\end{array}$ & $S M A Q P$ & RIC $95,9 b$ & C23785 & \\
\hline 282 & Valens & $364-367$ & E 3 & E & 20 & 2.07 & 7 & $\begin{array}{l}\text { DN VALEN-S } \\
\text { PF AVG }\end{array}$ & $\begin{array}{l}\text { SECVRITAS } \\
\text { REIPVBLICAE }\end{array}$ & $\begin{array}{l}\text { cres- } \\
\text { cent/--// } \\
\text { SM AQ P }\end{array}$ & RIC $95,9 b$ & G60-21 & \\
\hline 283 & Valens & $364-367$ & E 3 & $Æ$ & 18 & 2.26 & 6 & $\begin{array}{l}\text { DN VALEN-S } \\
\text { PF AVG }\end{array}$ & $\begin{array}{c}\text { SECVRITAS } \\
\text { REIPVBLICAE }\end{array}$ & "SM AQS & RIC 95, 9b & G60-107 & \\
\hline 284 & Valens & $364-367$ & E 3 & $F$ & 18 & 2.14 & 5 & $\begin{array}{l}\text { DN VALEN-S } \\
\text { [PF AVG] }\end{array}$ & $\begin{array}{c}\text { SECVRITAS } \\
\text { [REIPVBLI]CAE }\end{array}$ & $\begin{array}{c}B \cdot /--/ / S M \\
A Q P\end{array}$ & RIC $95,9 b$ & G60-187 & \\
\hline 285 & Valens & $364-367$ & Æ 3 & $F$ & 17 & 2.24 & 6 & $\begin{array}{l}\text { [DN V]ALEN- } \\
\text { [S PF AVG] }\end{array}$ & $\begin{array}{c}\text { SECVRITAS } \\
\text { [REIPVBLICAE] }\end{array}$ & $\begin{array}{c}\text { A-/--// } \\
\text { SMA[Q] ? }\end{array}$ & RIC $95,9 b$ & G60-256 & \\
\hline 286 & Valens & $364-367$ & Æ 3 & $F$ & 19 & 2.35 & 12 & $\begin{array}{l}\text { DN VALEN-S } \\
\text { PF AVG }\end{array}$ & $\begin{array}{l}\text { [SEC]VRITAS } \\
\text { REIPVBLICAE }\end{array}$ & $S M A Q ?$ & RIC $95,9 b$ & G61-58 & \\
\hline 287 & Valens & $364-367$ & E 3 & E & 19 & 1.91 & 6 & $\begin{array}{c}\text { DN [VALEN]-S } \\
\text { PF AVG }\end{array}$ & $\begin{array}{l}\text { SECVRITAS } \\
\text { REIPVBLICAE }\end{array}$ & $\begin{array}{c}B \cdot /-/ / S M \\
\text { AQS }\end{array}$ & RIC $95,9 b$ & G67-5 & \\
\hline 288 & Valens & $364-367$ & Æ 3 & FE & 18 & 2.72 & 7 & $\begin{array}{l}\text { DN VALEN-S } \\
\text { PF A[V]G }\end{array}$ & $\begin{array}{l}\text { SECVRITAS } \\
\text { REIPVBLICAE }\end{array}$ & B SIS C & RIC $146,7 b$ & C23920 & \\
\hline 289 & Valens & $364-367$ & $Æ_{3}$ & E & 18 & 2.47 & 11 & $\begin{array}{l}\text { DN VALEN-S } \\
\text { PF AVG }\end{array}$ & $\begin{array}{l}\text { SECVRITAS } \\
\text { REIPVBLICAE }\end{array}$ & "A SIS C & $\begin{array}{l}\text { RIC 146, } \\
7 b\end{array}$ & G60-3 & \\
\hline 290 & Valens & $364-367$ & $Æ_{3}$ & F & 17 & 2.24 & 7 & $\begin{array}{l}\text { DN VALEN-S } \\
\text { PF AVG }\end{array}$ & $\begin{array}{l}\text { SECVRITAS } \\
\text { REIPVBLICAE }\end{array}$ & $\begin{array}{c}* \mathrm{~A} /--/ / \mathrm{DA} \\
\text { SIS C }\end{array}$ & $\begin{array}{l}\text { RIC 146, } \\
7 b\end{array}$ & G60-31 & \\
\hline 291 & Valens & $364-367$ & $Æ 3$ & $\notin$ & 19 & 1.97 & 12 & $\begin{array}{l}\text { DN VALEN-S } \\
\text { PF AVG }\end{array}$ & $\begin{array}{l}\text { SECVRITAS } \\
\text { REIPVBLICAE }\end{array}$ & "A SIS C & $\begin{array}{c}\text { RIC 146, } \\
7 b\end{array}$ & G60-32 & \\
\hline 292 & Valens & $364-367$ & $Æ_{3}$ & F & 18 & 2.6 & 12 & $\begin{array}{l}\text { DN VALEN-S } \\
\text { PF AVG }\end{array}$ & $\begin{array}{l}\text { SECVRITAS } \\
\text { REIPVBLICAE }\end{array}$ & $\begin{array}{c}* \mathrm{~A} /--/ / \cdot \mathrm{A} \\
\mathrm{SISC}\end{array}$ & $\begin{array}{l}\text { RIC 146, } \\
7 b\end{array}$ & G60-66 & \\
\hline 293 & Valens & $364-367$ & $Æ_{3}$ & $\mathscr{E}$ & 18 & 2.07 & 1 & $\begin{array}{l}\text { DN VALEN-S } \\
\text { PF AVG }\end{array}$ & $\begin{array}{l}\text { [SECVRITAS] } \\
\text { REIPVBLICAE }\end{array}$ & $\begin{array}{c}* \mathrm{~A} /--/ / \mathrm{D} \mathrm{A} \\
\text { SIS C }\end{array}$ & $\begin{array}{l}\text { RIC 146, } \\
7 b\end{array}$ & G6o-68 & \\
\hline 294 & Valens & $364-367$ & $Æ_{3}$ & F & 18 & 2.46 & 6 & $\begin{array}{c}\text { DN VALEN-[S } \\
\text { PF AVG] }\end{array}$ & $\begin{array}{l}\text { SECVRITAS } \\
\text { REIPVBLICAE }\end{array}$ & $\begin{array}{c}* \mathrm{~A} /--/ / \cdot \mathrm{A} \\
\mathrm{SISC}\end{array}$ & $\begin{array}{c}\text { RIC } 146, \\
7 b\end{array}$ & G60-72 & \\
\hline 295 & Valens & $364-367$ & $Æ 3$ & F & 18 & 2.1 & 7 & $\begin{array}{c}\text { DN VALEN-S } \\
\text { PF AVG }\end{array}$ & $\begin{array}{c}\text { SECVRITAS } \\
\text { REIPVBLICAE }\end{array}$ & D A SIS C & $\begin{array}{c}\text { RIC 146, } \\
7 b\end{array}$ & G60-90 & \\
\hline 296 & Valens & $364-367$ & Æ 3 & $\mathscr{F}$ & 18 & 1.91 & 1 & $\begin{array}{l}\text { DN VALEN-S } \\
\text { PF AVG }\end{array}$ & $\begin{array}{l}\text { SECVRITAS } \\
\text { REIPVBLICAE }\end{array}$ & $\begin{array}{c}* \mathrm{~A}--/ / \mathrm{D} \mathrm{A} \\
{[\mathrm{SI}] \mathrm{SC}}\end{array}$ & $\begin{array}{l}\text { RIC 146, } \\
7 b\end{array}$ & G60-155 & \\
\hline 297 & Valens & $364-367$ & $Æ 3$ & E & 18 & 1.66 & 7 & $\begin{array}{c}\text { DN VALEN-S } \\
\text { PF AVG }\end{array}$ & $\begin{array}{c}\text { SECVRITAS } \\
\text { REIPVBLICAE] }\end{array}$ & $" \Delta \mathrm{SISC}$ & $\begin{array}{c}\text { RIC 146, } \\
7 b\end{array}$ & G60-168 & \\
\hline 298 & Valens & $364-367$ & E 3 & $F$ & 18 & 2.8 & 7 & $\begin{array}{l}\text { DN VALEN-S } \\
\text { PF AVG }\end{array}$ & $\begin{array}{l}\text { SECVRITAS } \\
\text { REIPVBLICAE }\end{array}$ & "A SIS C & $\begin{array}{l}\text { RIC 146, } \\
7 b\end{array}$ & G60-182 & \\
\hline 299 & Valens & $364-367$ & Æ 3 & FE & 18 & 2.27 & 1 & $\begin{array}{l}\text { DN VALEN-S } \\
\text { PF AVG }\end{array}$ & $\begin{array}{l}\text { SECVRITAS } \\
\text { REIPVBLICAE }\end{array}$ & $\begin{array}{c}--/ l . \\
\text { wreath//. A } \\
\text { SIS C }\end{array}$ & $\begin{array}{l}\text { RIC 146, } \\
7 \mathrm{~b}\end{array}$ & G60-205 & \\
\hline 300 & Valens & $364-367$ & Æ 3 & $F$ & 16 & 1.85 & 5 & $\begin{array}{l}\text { DN VALEN-S } \\
\text { PF AVG }\end{array}$ & $\begin{array}{l}\text { [SECVRITAS] } \\
\text { REIPVBLICAEE }\end{array}$ & $\begin{array}{c}* \mathrm{~A} /--/ / ? \\
\mathrm{SISC}\end{array}$ & $\begin{array}{l}\text { RIC } 146, \\
7 b\end{array}$ & G60-215 & \\
\hline 301 & Valens & $364-367$ & Æ 3 & $F$ & 17 & 1.64 & 6 & $\begin{array}{l}\text { DN VALEN-S } \\
\text { PF AVG }\end{array}$ & $\begin{array}{c}\text { SECVRITAS } \\
\text { REIPVB[LICAE] }\end{array}$ & $\begin{array}{l}* \mathrm{~A} /--/ / \text { ? } \\
\text { SIS C }\end{array}$ & $\begin{array}{l}\text { RIC } 146, \\
7 b\end{array}$ & G60-274 & \\
\hline 302 & Valens & $364-367$ & $Æ 3$ & 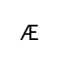 & 17 & 1.81 & 1 & $\begin{array}{c}\text { DN VALEN-[S } \\
\text { PF AVG] }\end{array}$ & $\begin{array}{c}\text { [SECVRITAS } \\
\text { REIPVBLICAE] }\end{array}$ & DA SIS C & $\begin{array}{c}\text { RIC } 146 \\
7 b\end{array}$ & G60-285 & \\
\hline 303 & Valens & $364-367$ & Æ 3 & $F$ & 18 & 1.95 & 12 & $\begin{array}{l}\text { DN VALEN-S } \\
\text { PF AVG }\end{array}$ & $\begin{array}{l}\text { SECVRITAS } \\
\text { REIPVBLICAE }\end{array}$ & $\begin{array}{c}* \mathrm{~A} /--/ / \mathrm{DA} \\
\text { SIS C }\end{array}$ & $\begin{array}{c}\text { RIC } 146, \\
7 b\end{array}$ & G61-21 & \\
\hline 304 & Valens & $364-367$ & Æ 3 & E & 19 & 1.97 & 12 & $\begin{array}{l}\text { DN VALEN-S } \\
\text { PF AVG }\end{array}$ & $\begin{array}{c}\text { SECVRITAS } \\
\text { REIPVBLICAE }\end{array}$ & - A SIS C & $\begin{array}{c}\text { RIC } 146 \\
7 b\end{array}$ & G61-54 & \\
\hline 305 & Valens & $364-367$ & Æ 3 & $F$ & 18 & 2.13 & 6 & $\begin{array}{l}\text { DN VALEN-S } \\
\text { PF AVG }\end{array}$ & $\begin{array}{l}\text { SECVRITAS } \\
\text { REIPVB[LICAE] }\end{array}$ & - A SIS C & $\begin{array}{l}\text { RIC } 146, \\
7 b\end{array}$ & G67-11 & \\
\hline 306 & Valens & $364-367$ & Æ 3 & E & 18 & 2.46 & 5 & $\begin{array}{l}\text { DN VALEN-S } \\
\text { PF AVG }\end{array}$ & $\begin{array}{c}\text { SECVRITAS } \\
\text { REIPVBLICAE }\end{array}$ & $* /--/ /$ TES A & $\begin{array}{c}\text { RIC 176, } \\
18 \mathrm{~b}\end{array}$ & G60-56 & \\
\hline 307 & Valens & $364-367$ & Æ 3 & E & 19 & 1.95 & 10 & $\begin{array}{l}\text { DN VALEN-S } \\
\text { PF AVG }\end{array}$ & $\begin{array}{l}\text { SECVRITAS } \\
\text { REIPVBLICAE }\end{array}$ & TES A & $\begin{array}{c}\text { RIC } 176 \\
18 \mathrm{~b}\end{array}$ & G60-106 & \\
\hline
\end{tabular}




\begin{tabular}{|c|c|c|c|c|c|c|c|c|c|c|c|c|c|}
\hline \multicolumn{14}{|c|}{ SOTIN } \\
\hline $\begin{array}{l}\text { CAT. } \\
\text { NO. }\end{array}$ & RULER & $\begin{array}{l}\text { DATING } \\
\text { (YRS) }\end{array}$ & DENOM. & MAT. & $\begin{array}{c}\mathrm{D} . \\
(\mathrm{mm})\end{array}$ & $\begin{array}{l}\text { WT. } \\
\text { (g) }\end{array}$ & $\begin{array}{l}\text { ORI- } \\
\text { ENT. }\end{array}$ & OBVERSE & REVERSE & $\begin{array}{c}\text { MINT } \\
\text { MARKS }\end{array}$ & $\begin{array}{l}\text { REFE- } \\
\text { RENCE }\end{array}$ & $\begin{array}{l}\text { INV. } \\
\text { NO. }\end{array}$ & NOTE \\
\hline 308 & Valens & $364-367$ & E 3 & Æ & 18 & 1.96 & 11 & $\begin{array}{c}\text { DN VALEN-S } \\
\text { PF AVG }\end{array}$ & $\begin{array}{l}\text { SECVRITAS } \\
\text { REIPVBLICAE }\end{array}$ & $\begin{array}{l}* /--/ / \\
\text { TESA }\end{array}$ & $\begin{array}{c}\text { RIC } 176 \\
18 \mathrm{~b}\end{array}$ & G60-119 & \\
\hline 309 & Valens & $364-367$ & E 3 & $Æ$ & 18 & 1.32 & 1 & $\begin{array}{c}\text { DN VALEN-S } \\
\text { PF AVG }\end{array}$ & $\begin{array}{c}\text { SECVRITAS } \\
\text { REIPVBLICAE }\end{array}$ & TES B & $\begin{array}{c}\text { RIC } 176 \\
18 \mathrm{~b}\end{array}$ & G60-201 & \\
\hline 310 & Valens & $364-367$ & E 3 & 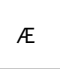 & 17 & 2.58 & 11 & $\begin{array}{l}\text { DN VALEN-[S } \\
\text { PF AVG] }\end{array}$ & $\begin{array}{l}\text { [SECVRITAS] } \\
\text { REIPVBLICAE }\end{array}$ & TES $\Delta$ & $\begin{array}{c}\text { RIC } 176 \\
18 \mathrm{~b}\end{array}$ & G60-230 & \\
\hline 311 & Valens & $364-367$ & E 3 & E & 17 & 1.89 & 11 & $\begin{array}{c}\text { DN VALEN-S } \\
\text { PF AV[G] }\end{array}$ & $\begin{array}{c}\text { SECVRITAS } \\
\text { [REIPVBLICAE] }\end{array}$ & TES ? & $\begin{array}{c}\text { RIC } 176 \\
18 \mathrm{~b}\end{array}$ & G60-234 & \\
\hline 312 & Valens & $364-367$ & E 3 & E & 18 & 1.8 & 11 & $\begin{array}{c}\text { DN VALEN-S } \\
\text { PF AVG }\end{array}$ & $\begin{array}{c}\text { SECVRITAS } \\
\text { REIPVBLICAE }\end{array}$ & TES A & $\begin{array}{c}\text { RIC } 176 \\
18 \mathrm{~b}\end{array}$ & G60-243 & \\
\hline 313 & Valens & $364-367$ & Æ 3 & 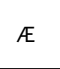 & 17 & 1.6 & 6 & $\begin{array}{c}\text { DN VALEN-S } \\
\text { PF AVG }\end{array}$ & $\begin{array}{l}\text { SECVRITAS } \\
\text { REIPVBLICAE }\end{array}$ & TES A & $\begin{array}{c}\text { RIC } 176 \\
18 \mathrm{~b}\end{array}$ & G61-24 & \\
\hline 314 & Valens & $364-367$ & E 3 & $Æ$ & 17 & 2.56 & 6 & $\begin{array}{l}\text { DN VALEN-[S } \\
\text { PF AVG] }\end{array}$ & $\begin{array}{c}\text { SECVRITAS } \\
\text { REI[PVBLICAE] }\end{array}$ & $* /--/ /$ TES 「 & $\begin{array}{c}\text { RIC } 176 \\
18 b\end{array}$ & G61-39 & \\
\hline 315 & Gratian & $364-367$ & E 3 & E & 17 & 2.19 & 5 & $\begin{array}{l}\text { DN GRATIA- } \\
\text { NVS PF AVG }\end{array}$ & $\begin{array}{l}\text { GLORIA RO-MA- } \\
\text { NORVM }\end{array}$ & $\begin{array}{c}\text { I. } \\
\text { wreath/·Г// } \\
\text { TES }\end{array}$ & $\begin{array}{c}\text { RIC } 176 \\
16 \mathrm{c}\end{array}$ & G61-26 & \\
\hline 316 & Valentinian I & $364-375$ & E 3 & E & 19 & 2.06 & 12 & $\begin{array}{l}\text { DN VALE[] } \\
\text { NTINI]-ANVS } \\
\text { PF AVG }\end{array}$ & $\begin{array}{l}\text { GLORIA RO-MA- } \\
\text { NORVM }\end{array}$ & $?$ & incerta & G60-162 & \\
\hline 317 & Valentinian I & $364-375$ & E 3 & 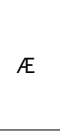 & 18 & 1.77 & 7 & $\begin{array}{c}\text { DN } \\
\text { VALEN[TINI- } \\
\text { ANVS] PF } \\
\text { AVG }\end{array}$ & $\begin{array}{l}\text { GLORIA RO-MA- } \\
\text { NORVM }\end{array}$ & $?$ & incerta & G60-214 & \\
\hline 318 & Valentinian I & $364-375$ & E 3 & 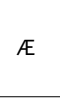 & 17 & 2.68 & 1 & $\begin{array}{c}\text { DN VALENTI- } \\
\text { NI-ANVS PF } \\
\text { AVG }\end{array}$ & $\begin{array}{c}\text { [G]LORIA RO[- } \\
\text { MANORVM] }\end{array}$ & $?$ & incerta & G60-222 & \\
\hline 319 & Valentinian I & $364-375$ & $\mathbb{E}_{3}$ & A & 18 & 2.2 & 12 & $\begin{array}{c}\text { DN VALENTI- } \\
\text { NI-[ANVS PF } \\
\text { AVG] }\end{array}$ & $\begin{array}{l}\text { GLORIA RO- } \\
\text { MANO[RVM] }\end{array}$ & $?$ & incerta & G60-260 & \\
\hline 320 & Valentinian I & $364-375$ & E 3 & Æ & 18 & 2.56 & 1 & $\begin{array}{c}\text { DN VALENTI- } \\
\text { NI-ANVS PF } \\
\text { AVG }\end{array}$ & $\begin{array}{l}\text { GLORIA RO-MA- } \\
\text { NORVM }\end{array}$ & $?$ & incerta & G61-41 & \\
\hline 321 & Valentinian I & $364-375$ & $\mathbb{E}_{3}$ & A & 18 & 1.57 & 12 & $\begin{array}{l}\text { DN VALENTI- } \\
\text { NI-ANVS [PF] } \\
\text { AVG }\end{array}$ & $\begin{array}{l}\text { [SE]CVRITAS } \\
\text { REIPVBLICAE }\end{array}$ & $?$ & incerta & G60-142 & \\
\hline 322 & Valentinian I & $364-375$ & E 3 & A & 17 & 1.41 & 11 & $\begin{array}{c}\text { [DN VALEN] } \\
\text { TINI-ANVS PF } \\
\text { AVG }\end{array}$ & $\begin{array}{c}\text { SECVRITAS } \\
\text { [REIPVBLICAE] }\end{array}$ & $?$ & incerta & G60-240 & \\
\hline 323 & Valentinian I & $364-375$ & $Æ_{3} 3$ & A & 17 & 1.79 & $?$ & $\begin{array}{c}\text { DN } \\
\text { [VALENTINI]- } \\
\text { ANVS PF AVG }\end{array}$ & $?$ & $?$ & incerta & G60-221 & \\
\hline 324 & Valentinian I & $364-375$ & $\mathbb{E}_{3}$ & 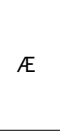 & 17 & 2.17 & 1 & $\begin{array}{c}\text { [DN } \\
\text { VALENTINI]- } \\
\text { ANVS PF AVG }\end{array}$ & $\begin{array}{l}\text { [GLOR]IA RO- } \\
\text { MANO[RVM] }\end{array}$ & SM KA & $\begin{array}{c}\text { RIC } \\
240 / 241 \\
8 a / 12 a\end{array}$ & G60-288 & $\begin{array}{l}\text { issues } \\
\text { indi- } \\
\text { stingu- } \\
\text { ishable }\end{array}$ \\
\hline 325 & Valentinian I & $364-375$ & $E_{3}$ & 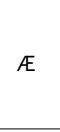 & 18 & 2.54 & 6 & $\begin{array}{c}\text { DN VALENTI- } \\
\text { NI-ANVS PF } \\
\text { AVG }\end{array}$ & $\begin{array}{l}\text { SECVRITAS } \\
\text { REIPVBLICAE }\end{array}$ & SM KA & $\begin{array}{l}\text { RIC 241, } \\
11 \mathrm{a} / 12 \mathrm{a}\end{array}$ & G60-101 & $\begin{array}{l}\text { issues } \\
\text { indi- } \\
\text { stingu- } \\
\text { ishable }\end{array}$ \\
\hline 326 & $\begin{array}{l}\text { Valent. I/ } \\
\text { Valens }\end{array}$ & $364-375$ & $E_{3}$ & 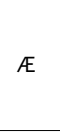 & 16 & 2.1 & 2 & $?$ & $\begin{array}{l}\text { [GLORIA RO- } \\
\text { MANORVM] }\end{array}$ & SM KA & $\begin{array}{c}\text { RIC } \\
240 / 241 \\
8 a-b / 12 a-b\end{array}$ & G60-255 & $\begin{array}{l}\text { issues } \\
\text { indi- } \\
\text { stingu- } \\
\text { ishable }\end{array}$ \\
\hline 327 & $\begin{array}{c}\text { Valent. I/ } \\
\text { Gratian }\end{array}$ & $364-375$ & $E_{3}$ & 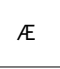 & 15 & 1.72 & 11 & $\begin{array}{c}\text { ? A[NV]S PF } \\
\text { AVG }\end{array}$ & $\begin{array}{c}\text { SECVRITA[S } \\
\text { REIPVBLICAE] }\end{array}$ & $?$ & incerta & G60-251 & \\
\hline 328 & Valens & $364-375$ & E 3 & 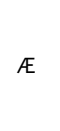 & 18 & 2.23 & 7 & $\begin{array}{c}\text { DN VALEN-S } \\
\text { PF AVG }\end{array}$ & $\begin{array}{c}\text { [SECVRITAS R] } \\
\text { EIPVBLICAE }\end{array}$ & SM K $\Delta$ & $\begin{array}{l}\text { RIC 241, } \\
11 b / 13 b\end{array}$ & G60-287 & $\begin{array}{l}\text { issues } \\
\text { indi- } \\
\text { stingu- } \\
\text { ishable }\end{array}$ \\
\hline 329 & Valens & $364-378$ & $\not E_{3}$ & A & 18 & 2.07 & 7 & $\begin{array}{c}\text { [DN] VALEN-S } \\
\text { PF AVG }\end{array}$ & $\begin{array}{l}\text { GLORIA RO-MA- } \\
\text { NORVM }\end{array}$ & $?$ & incerta & G 60-174 & \\
\hline 330 & Valens & $364-378$ & Æ 3 & $Æ$ & 18 & 2.34 & 7 & $\begin{array}{c}\text { [DN] VALEN-S } \\
\text { PF AVG }\end{array}$ & $\begin{array}{l}\text { GLORIA RO-MA- } \\
\text { NORVM }\end{array}$ & $?$ & incerta & G60-184 & \\
\hline 331 & Valens & $364-378$ & Æ 3 & A & 18 & 2.36 & 11 & $\begin{array}{l}\text { [DN VALEN] } \\
-S \text { PF A[VG] }\end{array}$ & $\begin{array}{l}\text { GLO[R]IA RO- } \\
\text { [MANORVM] }\end{array}$ & $?$ & incerta & G60-192 & \\
\hline
\end{tabular}




\begin{tabular}{|c|c|c|c|c|c|c|c|c|c|c|c|c|c|}
\hline \multicolumn{14}{|c|}{ SOTIN } \\
\hline $\begin{array}{l}\text { CAT. } \\
\text { NO. }\end{array}$ & RULER & $\begin{array}{l}\text { DATING } \\
\text { (YRS) }\end{array}$ & DENOM. & MAT. & $\begin{array}{c}\mathrm{D} . \\
(\mathrm{mm})\end{array}$ & $\begin{array}{l}\text { WT. } \\
\text { (g) }\end{array}$ & $\begin{array}{l}\text { ORI- } \\
\text { ENT. }\end{array}$ & OBVERSE & REVERSE & $\begin{array}{l}\text { MINT } \\
\text { MARKS }\end{array}$ & $\begin{array}{l}\text { REFE- } \\
\text { RENCE }\end{array}$ & $\begin{array}{l}\text { INV. } \\
\text { NO. }\end{array}$ & NOTE \\
\hline 332 & Valens & $364-378$ & E 3 & $\mathscr{E}$ & 18 & 1.39 & 7 & $\begin{array}{c}\text { [DN VALEN]-S } \\
\text { P[F AVG] }\end{array}$ & $\begin{array}{l}\text { [GLORIA RO- } \\
\text { MANORVM] }\end{array}$ & $?$ & incerta & G60-200 & \\
\hline 333 & Valens & $364-378$ & Æ 3 & $F$ & 18 & 0.12 & 2 & $\begin{array}{l}\text { DN VALEN-S } \\
\text { PF AVG }\end{array}$ & $\begin{array}{l}\text { [GLOR]IA RO- } \\
\text { MA[NORVM] }\end{array}$ & $?$ & incerta & G60-229 & \\
\hline 334 & Valens & $364-378$ & $Æ 3$ & $Æ$ & 18 & 1.91 & 5 & $\begin{array}{c}\text { [DN VALEN]-S } \\
\text { PF AVG }\end{array}$ & $\begin{array}{l}\text { GLORIA RO- } \\
\text { [MANORVM] }\end{array}$ & $?$ & incerta & G60-232 & \\
\hline 335 & Valens & $364-378$ & Æ3 & E & 17 & 0.83 & 12 & $\begin{array}{l}\text { [DN VALEN]-S } \\
\text { PF AVG }\end{array}$ & $\begin{array}{l}\text { GLORIA RO- } \\
\text { [MANORVM] }\end{array}$ & $?$ & incerta & G60-241 & \\
\hline 336 & Valens & $364-378$ & $\mathbb{E}_{3}$ & $\mathscr{E}$ & 17 & 1.82 & 6 & $\begin{array}{l}\text { DN V[A]LEN- } \\
{[S] \text { PF [AVG] }}\end{array}$ & $\begin{array}{l}\text { GLORIA RO- } \\
\text { [MANORVM] }\end{array}$ & $?$ & incerta & G60-261 & \\
\hline 337 & Valens & $364-378$ & $A_{3} 3$ & E & 19 & 0.6 & 6 & $\begin{array}{l}\text { [DN VALEN]-S } \\
\text { PF AVG }\end{array}$ & $\begin{array}{l}\text { [GLORIA RO- } \\
\text { MANORVM] }\end{array}$ & ? & incerta & G60-279 & \\
\hline 338 & Valens & $364-378$ & Æ 3 & $F$ & 17 & 1.18 & 6 & $\begin{array}{l}\text { [DN VALEN]-S } \\
\text { PF AVG }\end{array}$ & $\begin{array}{l}\text { [GLORIA RO]- } \\
\text { MANORVM }\end{array}$ & $--/ * / / ?$ & incerta & G61-63 & \\
\hline 339 & Valens & $364-378$ & Æ 3 & $\mathscr{F}$ & 18 & 1.69 & 7 & $\begin{array}{l}\text { DN VALEN-S } \\
\text { PF AVG }\end{array}$ & $\begin{array}{l}\text { SECVRITAS } \\
\text { REIPVBLICAE }\end{array}$ & $?$ & incerta & G60-151 & \\
\hline 340 & Valens & $364-378$ & Æ 3 & FE & 19 & 2.29 & 11 & $\begin{array}{l}\text { DN VALEN-[S } \\
\text { PF AVG ] }\end{array}$ & $\begin{array}{l}\text { [SECVRITAS } \\
\text { REIPVBLICAE] }\end{array}$ & $?$ & incerta & G60-167 & \\
\hline 341 & Valens & $364-378$ & $A_{3}$ & FE & 18 & 2.5 & 5 & $\begin{array}{l}\text { DN VALEN-S } \\
\text { PF AVG }\end{array}$ & $\begin{array}{c}\text { [SECVRITAS } \\
\text { REIPVBLICAE] }\end{array}$ & ? & incerta & G60-186 & \\
\hline 342 & Valens & $364-378$ & $Æ_{3}$ & $F$ & 17 & 2.89 & 10 & $\begin{array}{l}\text { DN VAL[EN]-S } \\
\text { PF AVG }\end{array}$ & $\begin{array}{l}\text { SECVRITAS[ } \\
\text { REIPVBLICAE] }\end{array}$ & ? & incerta & G60-190 & \\
\hline 343 & Valens & $364-375$ & $Æ_{3}$ & E & 19 & 1.83 & 6 & $\begin{array}{l}\text { [DN VALEN]-S } \\
\text { PF AVG }\end{array}$ & $\begin{array}{l}\text { [SECVRITAS] } \\
\text { REIPVBLICAE }\end{array}$ & $\begin{array}{c}\text { ?/--//? SM } \\
\text { AQS }\end{array}$ & $\begin{array}{c}\text { RIC } 95 / 96 \\
9 b / 12 b\end{array}$ & G60-197 & \\
\hline 344 & Valens & $364-378$ & Æ 3 & $\mathscr{F}$ & 18 & 2.43 & 11 & $\begin{array}{l}\text { DN VALEN-S } \\
\text { PF AVG }\end{array}$ & $\begin{array}{c}\text { SECVRITAS [REIP] } \\
\text { VB[LICAE] }\end{array}$ & ? & incerta & G60-216 & \\
\hline 345 & Valens & $364-378$ & $Æ_{3}$ & E & 17 & 2.47 & 12 & $\begin{array}{c}\text { DN VA[LEN]-S } \\
\text { PF AVG }\end{array}$ & $\begin{array}{c}\text { [SECVRITAS REI] } \\
\text { PVB[LICAE] }\end{array}$ & ? & incerta & G60-252 & \\
\hline 346 & Valens & $364-378$ & $Æ_{3}$ & E & 19 & 1.59 & 11 & $\begin{array}{l}\text { DN VALEN-[S } \\
\text { PF AVG] }\end{array}$ & $\begin{array}{l}\text { [SECVRITAS] } \\
\text { REIPVBLICAE }\end{array}$ & $?$ & incerta & G6o-257 & \\
\hline 347 & Valens & $364-378$ & $\mathbb{E}_{3}$ & E & 17 & 1.52 & 5 & $\begin{array}{l}\text { DN VALEN-S } \\
\text { PF AVG }\end{array}$ & $\begin{array}{l}\text { [SECV]RITAS } \\
\text { [REIPV]BLICAE }\end{array}$ & ? & incerta & G60-259 & \\
\hline 348 & Valens & $364-378$ & $Æ_{3}$ & E & 17 & 2.94 & 5 & $\begin{array}{l}\text { DN VALEN-S } \\
\text { PF AVG }\end{array}$ & $\begin{array}{c}\text { [SECVRITAS } \\
\text { REIPVBLICAE] }\end{array}$ & $*|-| /$ ? & incerta & G60-275 & \\
\hline 349 & Valens & $364-378$ & $Æ_{3}$ & E & 17 & 1.76 & 11 & $\begin{array}{l}\text { [DN VALEN]-S } \\
\text { PF AVG }\end{array}$ & $\begin{array}{l}\text { SECVRITAS } \\
\text { REIPV[BLICAE] }\end{array}$ & ? & incerta & G60-280 & \\
\hline 350 & Valens & $364-375$ & $Æ_{3} 3$ & E & 19 & 2.01 & 6 & $\begin{array}{l}\text { DN VALEN-[S } \\
\text { PF AVG] }\end{array}$ & $\begin{array}{l}\text { SECVRITAS } \\
\text { REIPV[BLICAE] }\end{array}$ & ? SM AQS & $\begin{array}{c}\text { RIC } 95 / 96 \\
9 b / 12 b\end{array}$ & G60-286 & \\
\hline 351 & Valens & $364-375$ & $\notin_{3}$ & E & 17 & 2.71 & 5 & $\begin{array}{l}\text { [DN] VALEN-S } \\
\text { PF AVG }\end{array}$ & $\begin{array}{c}\text { SECVRITAS } \\
\text { [REIPVBLICAE] }\end{array}$ & ALE A & $\begin{array}{c}\text { RIC } \\
298 / 299 \\
3 a / 5 b\end{array}$ & G61-23 & $\begin{array}{l}\text { issues } \\
\text { indi- } \\
\text { stingu- } \\
\text { ishable }\end{array}$ \\
\hline 352 & Valens & $364-378$ & $Æ_{3}$ & E & 18 & 1.9 & 11 & $\begin{array}{l}\text { D[N VALE]N-S } \\
\text { PF AVG }\end{array}$ & $\begin{array}{l}\text { [SEC]VRITAS } \\
\text { REIPVBLIC[AE] }\end{array}$ & $?$ & incerta & G68-1 & \\
\hline 353 & Gratian & $364-378$ & $Æ_{3} 3$ & E & 18 & 1.81 & 1 & $\begin{array}{c}\text { DN } \\
\text { GRATANV[S } \\
\text { PF AVG] }\end{array}$ & $\begin{array}{l}\text { GLORIA RO- } \\
\text { [MANORVM] }\end{array}$ & $?$ & incerta & G60-178 & \\
\hline 354 & $\begin{array}{l}\text { Valent. I- } \\
\text { Valent. II }\end{array}$ & $364-378$ & $\notin_{3} 3$ & E & 19 & 1.99 & 5 & ? & $\begin{array}{l}\text { [GLORIA RO- } \\
\text { MANORVM] }\end{array}$ & TES & incerta & G61-44 & \\
\hline 355 & $\begin{array}{l}\text { Valent. I } \\
\text { - Arcadius }\end{array}$ & $364-392$ & $\notin_{3} 3$ & E & 15 & 1.77 & 5 & ? & $\begin{array}{l}\text { [GLORIA RO- } \\
\text { MANORVM] }\end{array}$ & $?$ & incerta & G60-218 & \\
\hline 356 & Valentinian I & $367-375$ & $\notin_{3} 3$ & EE & 18 & 2.21 & 5 & $\begin{array}{c}\text { DN } \\
\text { VALEN[TINI]- } \\
\text { ANVS PF } \\
\text { AVGG }\end{array}$ & $\begin{array}{c}\text { GLORIA RO[-MA] } \\
\text { NORVM }\end{array}$ & $\begin{array}{c}\text { O/FII/pol// } \\
\text { LVG[S] }\end{array}$ & $\begin{array}{l}\text { RIC } 46 \\
20 a\end{array}$ & C22733 & \\
\hline 357 & Valentinian I & $367-375$ & $Æ_{3}$ & EE & 18 & 2.06 & 1 & $\begin{array}{c}\text { DN } \\
\text { VALENTIN[I- } \\
\text { AN]VS PF } \\
\text { AVG }\end{array}$ & $\begin{array}{l}\text { [G]LORI[A RO]- } \\
\text { MANORVM }\end{array}$ & $\begin{array}{c}--/ / \text {. wre- } \\
\text { ath//[SM] } \\
\text { AQP }\end{array}$ & $\begin{array}{l}\text { RIC 96, } \\
\text { 11a }\end{array}$ & G61-51 & \\
\hline 358 & Valentinian I & $367-375$ & $\mathbb{E}_{3}$ & E & 18 & 2.3 & 6 & $\begin{array}{c}\text { DN VALENTI- } \\
\text { NI-ANVS PF } \\
\text { AVG }\end{array}$ & $\begin{array}{l}\text { GLORIA RO-MA- } \\
\text { NORVM }\end{array}$ & $\begin{array}{l}\mathrm{Q} / * \mathrm{RO} / / \mathrm{B} \\
\mathrm{SISC} 4\end{array}$ & $\begin{array}{l}\text { RIC 147, } \\
14 \mathrm{a}\end{array}$ & C22867 & \\
\hline 359 & Valentinian I & $367-375$ & $\notin_{3} 3$ & EE & 18 & 2.4 & 12 & $\begin{array}{l}\text { DN VALENTI- } \\
\text { NI-ANVS PF } \\
\text { AVG }\end{array}$ & $\begin{array}{l}\text { GLORIA RO-MA- } \\
\text { NORVM }\end{array}$ & $\begin{array}{c}--/ D / / \\
\text { crescent B } \\
\text { SIS C }\end{array}$ & $\begin{array}{l}\text { RIC 147, } \\
14 \mathrm{a}\end{array}$ & G60-14 & \\
\hline
\end{tabular}




\begin{tabular}{|c|c|c|c|c|c|c|c|c|c|c|c|c|c|}
\hline \multicolumn{14}{|c|}{ SOTIN } \\
\hline $\begin{array}{l}\text { CAT. } \\
\text { NO. }\end{array}$ & RULER & $\begin{array}{l}\text { DATING } \\
\text { (YRS) }\end{array}$ & DENOM. & MAT. & $\begin{array}{c}\text { D. } \\
(\mathrm{mm})\end{array}$ & $\begin{array}{l}\text { WT. } \\
(\mathrm{g})\end{array}$ & $\begin{array}{l}\text { ORI- } \\
\text { ENT. }\end{array}$ & OBVERSE & REVERSE & $\begin{array}{l}\text { MINT } \\
\text { MARKS }\end{array}$ & $\begin{array}{l}\text { REFE- } \\
\text { RENCE }\end{array}$ & $\begin{array}{l}\text { INV. } \\
\text { NO. }\end{array}$ & NOTE \\
\hline 360 & Valentinian I & $367-375$ & E 3 & $\mathbb{E}$ & 18 & 1.62 & 1 & $\begin{array}{c}\text { DN VALENTI- } \\
\text { NI-ANVS PF } \\
\text { AVG }\end{array}$ & $\begin{array}{l}\text { GLORIA RO-MA- } \\
\text { NORVM }\end{array}$ & $\begin{array}{l}\text { S./RÁ//B } \\
\text { SIS C4 }\end{array}$ & $\begin{array}{l}\text { RIC 147, } \\
\text { 14a }\end{array}$ & G60-26 & \\
\hline 361 & Valentinian I & $367-375$ & E3 & E & 18 & 2.08 & 7 & $\begin{array}{c}\text { DN VALENTI- } \\
\text { NI-ANVS PF } \\
\text { AVG }\end{array}$ & $\begin{array}{l}\text { GLORIA RO-MA- } \\
\text { NORVM }\end{array}$ & $\begin{array}{c}\mathrm{F} / \mathrm{Rk} / / \mathrm{B} \text { SIS } \\
\mathrm{C} 4\end{array}$ & $\begin{array}{l}\text { RIC 147, } \\
14 \mathrm{a}\end{array}$ & G60-27 & \\
\hline 362 & Valentinian I & $367-375$ & E 3 & $\mathbb{E}$ & 18 & 1.9 & 7 & $\begin{array}{c}\text { DN } \\
\text { VALEN[TINI]- } \\
\text { ANVS PF AVG }\end{array}$ & $\begin{array}{l}\text { GLORIA RO- } \\
\text { MANO[RVM] }\end{array}$ & $\begin{array}{c}--/ D / / * B \\
\text { SIS C }\end{array}$ & $\begin{array}{l}\text { RIC 147, } \\
14 \mathrm{a}\end{array}$ & G60-41 & \\
\hline 363 & Valentinian I & $367-375$ & E 3 & E & 18 & 2.25 & 6 & $\begin{array}{c}\text { DN VALENTI- } \\
\text { NI-ANVS PF } \\
\text { AVG }\end{array}$ & $\begin{array}{l}\text { GLORIA RO-MA- } \\
\text { NORVM }\end{array}$ & $\begin{array}{c}\mathrm{M} / * \mathrm{~F} / / \mathrm{B} \\
\mathrm{SISC}\end{array}$ & $\begin{array}{l}\text { RIC 147, } \\
14 \mathrm{a}\end{array}$ & G60-47 & \\
\hline 364 & Valentinian I & $367-375$ & $\mathbb{E}_{3}$ & E & 17 & 2.4 & 7 & $\begin{array}{c}\text { DN VALENTI- } \\
\text { NI-ANVS PF } \\
\text { AVG }\end{array}$ & $\begin{array}{l}\text { GLORIA RO-MA- } \\
\text { NORVM }\end{array}$ & $\begin{array}{l}\text { S/D//B } \\
\text { SIS C }\end{array}$ & $\begin{array}{l}\text { RIC 147, } \\
14 \mathrm{a}\end{array}$ & G60-51 & \\
\hline 365 & Valentinian I & $367-375$ & E 3 & $\mathbb{E}$ & 18 & 1.9 & 6 & $\begin{array}{c}\text { DN VALENTI- } \\
\text { NI-ANVS PF } \\
\text { AVG }\end{array}$ & $\begin{array}{l}\text { GLORIA RO-MA- } \\
\text { NORVM }\end{array}$ & $\begin{array}{c}\mathrm{M} / * \mathrm{P} / / \mathrm{B} \\
\mathrm{SISC}\end{array}$ & $\begin{array}{l}\text { RIC 147, } \\
14 \mathrm{a}\end{array}$ & G60-63 & \\
\hline 366 & Valentinian I & $367-375$ & E 3 & E & 18 & 1.61 & 1 & $\begin{array}{c}\text { DN VALENTI- } \\
\text { NI-ANVS PF } \\
\text { AVG }\end{array}$ & $\begin{array}{l}\text { GLORIA RO-MA- } \\
\text { NORVM }\end{array}$ & $\begin{array}{c}--/ D / / . \\
\text { crescent B } \\
\text { SIS C }\end{array}$ & $\begin{array}{l}\text { RIC 147, } \\
14 \mathrm{a}\end{array}$ & G60-75 & \\
\hline 367 & Valentinian I & $367-375$ & Æ 3 & $\mathbb{E}$ & 17 & 1.93 & 1 & $\begin{array}{c}\text { DN VALENTI- } \\
\text { NI-ANVS PF } \\
\text { AVG }\end{array}$ & $\begin{array}{l}\text { GLORIA RO-MA- } \\
\text { NORVM }\end{array}$ & $\begin{array}{l}--/ R / / \cdot B \\
\text { SIS C }\end{array}$ & $\begin{array}{l}\text { RIC 147, } \\
\text { 14a }\end{array}$ & G60-79 & \\
\hline 368 & Valentinian I & $367-375$ & $\mathbb{E}_{3}$ & $\mathbb{E}$ & 17 & 2.21 & 6 & $\begin{array}{c}\text { DN VALENTI- } \\
\text { NI-ANVS PF } \\
\text { AVG }\end{array}$ & $\begin{array}{l}\text { GLOR[IA RO]- } \\
\text { MANORVM }\end{array}$ & $\begin{array}{l}\mathrm{M} / * \mathrm{~F} / / \mathrm{B} \\
\mathrm{SISC}\end{array}$ & $\begin{array}{l}\text { RIC 147, } \\
14 \mathrm{a}\end{array}$ & G60-96 & \\
\hline 369 & Valentinian I & $367-375$ & E 3 & $\mathbb{E}$ & 17 & 2.23 & 12 & $\begin{array}{c}\text { DN VALENTI- } \\
\text { NI-ANVS PF } \\
\text { AVG }\end{array}$ & $\begin{array}{l}\text { GLORIA RO-MA- } \\
\text { NORVM }\end{array}$ & $\begin{array}{l}\mathrm{M} / * \mathrm{~F} / / \mathrm{B} \\
\mathrm{SISC}\end{array}$ & $\begin{array}{l}\text { RIC 147, } \\
\text { 14a }\end{array}$ & G60-110 & \\
\hline 370 & Valentinian I & $367-375$ & $\mathbb{E}_{3}$ & $\mathbb{E}$ & 17 & 2.61 & 12 & $\begin{array}{c}\text { DN VALENTI- } \\
\text { NI-ANVS PF } \\
\text { AVG }\end{array}$ & $\begin{array}{l}\text { GLORIA RO-MA- } \\
\text { NORVM }\end{array}$ & $\begin{array}{c}\mathrm{M} / * \mathrm{P} / / \mathrm{B} \\
\mathrm{SISC}\end{array}$ & $\begin{array}{l}\text { RIC 147, } \\
\text { 14a }\end{array}$ & G60-126 & \\
\hline 371 & Valentinian I & $367-375$ & E 3 & $\mathbb{E}$ & 18 & 2.44 & 6 & $\begin{array}{c}\text { DN VALENTI- } \\
\text { NI-[ANVS PF } \\
\text { AVG] }\end{array}$ & $\begin{array}{l}\text { GLORIA RO- } \\
\text { [MANORVM] }\end{array}$ & $\begin{array}{l}--/ R / / \cdot B \\
\text { SIS C }\end{array}$ & $\begin{array}{l}\text { RIC 147, } \\
\quad 14 \mathrm{a}\end{array}$ & G60-132 & \\
\hline 372 & Valentinian I & $367-375$ & $E_{3}$ & $\notin$ & 18 & 2.1 & 11 & $\begin{array}{c}\text { DN VALENTI- } \\
\text { NI-ANVS PF } \\
\text { AVG }\end{array}$ & $\begin{array}{l}\text { GLORIA RO-MA- } \\
\text { NORVM }\end{array}$ & $\begin{array}{c}--/ R / /[\cdot] \mathrm{B} \\
\mathrm{SISC}\end{array}$ & $\begin{array}{l}\text { RIC 147, } \\
\text { 14a }\end{array}$ & G60-137 & \\
\hline 373 & Valentinian I & $367-375$ & Æ3 & $\mathbb{E}$ & 19 & 2.54 & 6 & $\begin{array}{c}\text { DN VALENTI- } \\
\text { NI-ANVS PF } \\
\text { AVG }\end{array}$ & $\begin{array}{l}\text { GLORIA RO-MA- } \\
\text { NORVM }\end{array}$ & $\begin{array}{c}\mathrm{M} / * \mathrm{P} / / \mathrm{B} \\
\mathrm{SISC}\end{array}$ & $\begin{array}{l}\text { RIC 147, } \\
\text { 14a }\end{array}$ & G60-141 & \\
\hline 374 & Valentinian I & $367-375$ & $Æ_{3} 3$ & $\mathbb{E}$ & 17 & 2.27 & 6 & $\begin{array}{c}\text { DN } \\
\text { VAL[ENTINI]- } \\
\text { ANVS PF AVG }\end{array}$ & $\begin{array}{l}\text { GLORIA RO- } \\
\text { [MANORVM] }\end{array}$ & $\begin{array}{l}\mathrm{M} / * \mathrm{P} / / \mathrm{B} \\
\mathrm{SISC}\end{array}$ & $\begin{array}{l}\text { RIC 147, } \\
\text { 14a }\end{array}$ & G60-148 & \\
\hline 375 & Valentinian I & $367-375$ & $\mathbb{E}_{3}$ & E & 18 & 2.88 & 7 & $\begin{array}{c}\text { DN } \\
\text { VALEN[TINI- } \\
\text { ANVS PF } \\
\text { AVG] }\end{array}$ & $\begin{array}{l}\text { GLORIA RO-MA- } \\
\text { NORVM }\end{array}$ & $\begin{array}{l}--/ R / / \cdot \Gamma \\
\text { SIS C }\end{array}$ & $\begin{array}{l}\text { RIC 147, } \\
\text { 14a }\end{array}$ & G60-173 & \\
\hline 376 & Valentinian I & $367-375$ & $Æ_{3}$ & $\mathbb{E}$ & 18 & 2.12 & 12 & $\begin{array}{c}\text { DN } \\
\text { VALE[NTINI- } \\
\text { ANVS PF] } \\
\text { AVG }\end{array}$ & $\begin{array}{l}\text { [GLORIA] RO- } \\
\text { MANORVM }\end{array}$ & $\begin{array}{l}--/ R / / \cdot B \\
\text { SIS C }\end{array}$ & $\begin{array}{l}\text { RIC 147, } \\
\text { 14a }\end{array}$ & G60-177 & \\
\hline 377 & Valentinian I & $367-375$ & $\mathbb{E}_{3}$ & $\mathbb{E}$ & 17 & 2.68 & 5 & $\begin{array}{l}\text { DN VALENTI- } \\
\text { NI-ANVS PF } \\
\text { AVG }\end{array}$ & $\begin{array}{l}\text { GLORIA RO-MA- } \\
\text { NORVM }\end{array}$ & $\begin{array}{c}\text { Q-/?//B SIS } \\
C V\end{array}$ & $\begin{array}{l}\text { RIC 147, } \\
\text { 14a }\end{array}$ & G60-238 & \\
\hline 378 & Valentinian I & $367-375$ & E3 & 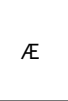 & 18 & 1.72 & 7 & $\begin{array}{c}\text { [DN } \\
\text { VALENTINI]- } \\
\text { ANVS PF AVG }\end{array}$ & $\begin{array}{l}\text { [GLO]RIA RO- } \\
\text { MAN[ORVM] }\end{array}$ & $\begin{array}{l}--/ R / / \cdot ? \\
\text { SIS C }\end{array}$ & $\begin{array}{l}\text { RIC 147, } \\
\quad 14 \mathrm{a}\end{array}$ & G60-276 & \\
\hline 379 & Valentinian I & $367-375$ & $E_{3}$ & $\mathbb{E}$ & 18 & 2.69 & 7 & $\begin{array}{c}\text { DN } \\
\text { VAL[ENTINI-] } \\
\text { ANVS PF AVG }\end{array}$ & $\begin{array}{l}\text { GLORIA RO-MA- } \\
\text { NORVM }\end{array}$ & $\begin{array}{l}\text { S/D//B } \\
\text { SIS C }\end{array}$ & $\begin{array}{l}\text { RIC 147, } \\
\text { 14a }\end{array}$ & G61-5 & \\
\hline 380 & Valentinian I & $367-375$ & $Æ_{3} 3$ & 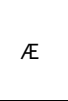 & 18 & 2.48 & 7 & $\begin{array}{c}\text { DN VALENTI- } \\
\text { NI-ANVS PF } \\
\text { AVG }\end{array}$ & $\begin{array}{l}\text { GLORIA RO-MA- } \\
\text { NORVM }\end{array}$ & $\begin{array}{l}--/ R / / \cdot B \\
\text { SIS C }\end{array}$ & $\begin{array}{l}\text { RIC 147, } \\
14 \mathrm{a}\end{array}$ & G67-6 & \\
\hline 381 & Valentinian I & $367-375$ & E 3 & $\mathscr{E}$ & 17 & 1.97 & 6 & $\begin{array}{l}\text { DN VALENTI- } \\
\text { NI-ANVS PF } \\
\text { AVG }\end{array}$ & $\begin{array}{l}\text { GLORIA RO-MA- } \\
\text { NORVM }\end{array}$ & $\begin{array}{c}\mathrm{M} / * \mathrm{~F} / / \mathrm{B} \\
\mathrm{SISC}\end{array}$ & $\begin{array}{l}\text { RIC 147, } \\
14 \mathrm{a}\end{array}$ & G67-8 & \\
\hline
\end{tabular}




\begin{tabular}{|c|c|c|c|c|c|c|c|c|c|c|c|c|c|}
\hline \multicolumn{14}{|c|}{ SOTIN } \\
\hline $\begin{array}{l}\text { CAT. } \\
\text { NO. }\end{array}$ & RULER & $\begin{array}{l}\text { DATING } \\
\text { (YRS) }\end{array}$ & DENOM. & MAT. & $\begin{array}{c}\text { D. } \\
(\mathrm{mm})\end{array}$ & $\begin{array}{l}\text { WT. } \\
\text { (g) }\end{array}$ & $\begin{array}{l}\text { ORI- } \\
\text { ENT. }\end{array}$ & OBVERSE & REVERSE & $\begin{array}{l}\text { MINT } \\
\text { MARKS }\end{array}$ & $\begin{array}{l}\text { REFE- } \\
\text { RENCE }\end{array}$ & $\begin{array}{l}\text { INV. } \\
\text { NO. }\end{array}$ & NOTE \\
\hline 382 & Valentinian I & $367-375$ & Æ 3 & $\notin$ & 19 & 2.62 & 2 & $\begin{array}{l}\text { DN VALENTI- } \\
\text { NI-ANVS PF } \\
\text { AVG }\end{array}$ & $\begin{array}{l}\text { GLORIA RO-MA- } \\
\text { NORVM }\end{array}$ & $\begin{array}{l}\mathrm{M} / * \mathrm{P} / / \mathrm{B} \\
\mathrm{SISC}\end{array}$ & $\begin{array}{l}\text { RIC 147, } \\
14 \mathrm{a}\end{array}$ & G61-11 & \\
\hline 383 & Valentinian I & $367-375$ & E 3 & $\notin$ & 17 & 2.55 & 1 & $\begin{array}{l}\text { DN VALENTI- } \\
\text { NI-ANVS PF } \\
\text { AVG }\end{array}$ & $\begin{array}{c}\text { GLORIA RO-MA- } \\
\text { NORVM }\end{array}$ & $\begin{array}{l}\mathrm{M} / * \mathrm{P} / / \mathrm{B} \\
\mathrm{SISC}\end{array}$ & $\begin{array}{l}\text { RIC } 147, \\
14 \mathrm{a}\end{array}$ & G61-12 & \\
\hline 384 & Valentinian I & $367-375$ & A 3 & $\notin$ & 18 & 2.74 & 7 & $\begin{array}{l}\text { DN VALENTI- } \\
\text { NI-ANVS PF } \\
\text { AVG }\end{array}$ & $\begin{array}{l}\text { GLORIA RO- } \\
\text { MANO[RVM] }\end{array}$ & $\begin{array}{l}--/ R / / \cdot B \\
\text { SIS C }\end{array}$ & $\begin{array}{l}\text { RIC 147, } \\
14 \mathrm{a}\end{array}$ & G61-16 & \\
\hline 385 & Valentinian I & $367-375$ & E 3 & $E$ & 18 & 2.28 & 7 & $\begin{array}{l}\text { DN VALENTI- } \\
\text { NI-ANVS PF } \\
\text { AVG }\end{array}$ & $\begin{array}{l}\text { GLORIA RO-MA- } \\
\text { NORVM }\end{array}$ & $\begin{array}{l}--/ D / / * B \\
\text { SIS C }\end{array}$ & $\begin{array}{l}\text { RIC 147, } \\
14 \mathrm{a}\end{array}$ & G61-29 & \\
\hline 386 & Valentinian I & $367-375$ & E 3 & $E$ & 20 & 2.2 & 1 & $\begin{array}{l}\text { DN VALENTI- } \\
\text { NI-ANVS PF } \\
\text { AVG }\end{array}$ & $\begin{array}{l}\text { GLORIA RO-MA- } \\
\text { NORVM }\end{array}$ & $\begin{array}{c}\mathrm{M} / * \mathrm{PI} / / \mathrm{B} \\
\mathrm{SISC}\end{array}$ & $\begin{array}{l}\text { RIC } 147 \\
14 \mathrm{a}\end{array}$ & G61-38 & \\
\hline 387 & Valentinian I & $367-375$ & $\mathbb{E}_{3}$ & $\notin$ & 18 & 2.15 & 1 & $\begin{array}{c}\text { [DN } \\
\text { VALENTINI]- } \\
\text { ANVS PF AVG }\end{array}$ & $\begin{array}{l}\text { GLORIA RO-MA- } \\
\text { NORVM }\end{array}$ & $\begin{array}{l}\mathrm{S} / * \mathrm{D} / / \mathrm{B} \\
\mathrm{SISC}\end{array}$ & $\begin{array}{l}\text { RIC 147, } \\
14 \mathrm{a}\end{array}$ & G61-52 & \\
\hline 388 & Valentinian I & $367-375$ & Æ 3 & $E$ & 18 & 2.7 & 7 & $\begin{array}{l}\text { DN VALENTI- } \\
\text { NI-ANVS PF } \\
\text { AVG }\end{array}$ & $\begin{array}{l}\text { GLORIA RO-MA- } \\
\text { NORVM }\end{array}$ & $\begin{array}{c}--/ D / / \\
\text { crescent B } \\
\text { SIS C }\end{array}$ & $\begin{array}{l}\text { RIC } 147 \\
14 \mathrm{a}\end{array}$ & G61-65 & \\
\hline 389 & Valentinian I & $367-375$ & Æ 3 & $\notin$ & 18 & 2.2 & 12 & $\begin{array}{c}\text { [DN } \\
\text { VALENTINI]- } \\
\text { ANVS PF AVG }\end{array}$ & $\begin{array}{l}\text { GLORIA RO- } \\
\text { [MANORVM] }\end{array}$ & $\begin{array}{l}--/ R / / \cdot B \\
\text { SIS C }\end{array}$ & $\begin{array}{l}\text { RIC 147, } \\
14 \mathrm{a}\end{array}$ & G61-67 & \\
\hline 390 & Valentinian I & $367-375$ & Æ 3 & $\mathbb{E}$ & 18 & 2.59 & 12 & $\begin{array}{l}\text { DN VALENTI- } \\
\text { NI-ANVS PF } \\
\text { AVG }\end{array}$ & $\begin{array}{c}\text { GLORIA RO-MA- } \\
\text { NORVM }\end{array}$ & $* / * \mathrm{~B} / / \mathrm{TES}$ & $\begin{array}{c}\text { RIC 178 } \\
26 \mathrm{a}\end{array}$ & C22959 & \\
\hline 391 & Valentinian I & $367-375$ & E 3 & $\notin$ & 18 & 2.28 & 6 & $\begin{array}{c}\text { DN VALENTI- } \\
\text { NI-ANVS PF } \\
\text { AVG }\end{array}$ & $\begin{array}{c}\text { GLORIA RO-MA- } \\
\text { NORVM }\end{array}$ & $* / * \mathrm{~B} / / \mathrm{TES}$ & $\begin{array}{c}\text { RIC 178 } \\
26 \mathrm{a}\end{array}$ & C22960 & \\
\hline 392 & Valentinian I & $367-375$ & Æ 3 & $\notin$ & 19 & 1.99 & 5 & $\begin{array}{c}\text { DN } \\
\text { VALEN[TINI]- } \\
\text { ANVS PF AVG }\end{array}$ & $\begin{array}{c}\text { GLORIA RO-[MA] } \\
\text { NORVM }\end{array}$ & $* / * \Delta / /$ TES & $\begin{array}{c}\text { RIC 178 } \\
26 \mathrm{a}\end{array}$ & C22967 & \\
\hline 393 & Valentinian I & $367-375$ & $\notin 3$ & E & 20 & 1.9 & 1 & $\begin{array}{l}\text { DN VALENTI- } \\
\text { NI-ANVS PF } \\
\text { AVG }\end{array}$ & $\begin{array}{l}\text { [GLOR]IA RO- } \\
\text { MANORVM }\end{array}$ & $\mathrm{V} / * \mathrm{~B} / / \mathrm{TES}$ & $\begin{array}{c}\text { RIC 178 } \\
26 a\end{array}$ & C22990 & \\
\hline 394 & Valentinian I & $367-375$ & Æ 3 & $\mathbb{E}$ & 19 & 2.1 & 11 & $\begin{array}{l}\text { DN VALENTI- } \\
\text { NI-ANVS PF } \\
\text { AVG }\end{array}$ & $\begin{array}{l}\text { GLORIA RO-MA- } \\
\text { NORVM }\end{array}$ & $\mathrm{Z} / * \mathrm{~A} / / \mathrm{TES}$ & $\begin{array}{c}\text { RIC } 178 \\
26 a\end{array}$ & C22997 & \\
\hline 395 & Valentinian I & $367-375$ & $Æ_{3} 3$ & $\notin$ & 17 & 2.31 & 11 & $\begin{array}{c}\text { DN } \\
\text { VALENTIN[I]- } \\
\text { ANVS PF AVG }\end{array}$ & $\begin{array}{l}\text { [GL]ORIA RO- } \\
\text { MANORVM }\end{array}$ & $\begin{array}{l}\text { I. wreath } \\
\text { /.A//[TES] }\end{array}$ & $\begin{array}{l}\text { RIC } 178 \\
26 a\end{array}$ & G60-15 & \\
\hline 396 & Valentinian I & $367-375$ & Æ 3 & $\notin$ & 19 & 1.91 & 6 & $\begin{array}{l}\text { DN VALENTI- } \\
\text { NI-ANVS PF } \\
\text { AVG }\end{array}$ & $\begin{array}{l}\text { GLORIA RO-MA- } \\
\text { NORVM }\end{array}$ & $* / * \mathrm{~B} / / \mathrm{TES}$ & $\begin{array}{l}\text { RIC 178, } \\
26 a\end{array}$ & G60-40 & \\
\hline 397 & Valentinian I & $367-375$ & $Æ_{3}$ & $\notin$ & 18 & 2.2 & 11 & $\begin{array}{l}\text { DN VALENTI- } \\
\text { NI-ANVS PF } \\
\text { AVG }\end{array}$ & $\begin{array}{l}\text { [GLO]RIA RO- } \\
\text { MANORVM }\end{array}$ & $--/ * \mathrm{~B} / / \mathrm{TES}$ & $\begin{array}{c}\text { RIC 178, } \\
26 a\end{array}$ & G60-140 & \\
\hline 398 & Valentinian I & $367-375$ & Æ 3 & $\notin$ & 17 & 2.94 & 5 & $\begin{array}{c}\text { [DN VALENTI- } \\
\text { NI-ANV]S PF } \\
\text { A[VG] }\end{array}$ & $\begin{array}{l}\text { [GLORIA RO- } \\
\text { MANORVM] }\end{array}$ & $\begin{array}{c}\text { I. } \\
\text { wreath/A// } \\
\text { TES }\end{array}$ & $\begin{array}{c}\text { RIC 178 } \\
26 \mathrm{a}\end{array}$ & G61-40 & \\
\hline 399 & Valentinian I & $367-375$ & $Æ_{3} 3$ & $\notin$ & 17 & 1.46 & 11 & $\begin{array}{c}\text { DN } \\
\text { VALEN[TINI- } \\
\text { ANVS PF } \\
\text { AVG] }\end{array}$ & $\begin{array}{l}\text { GLOR[IA RO- } \\
\text { MANORVM] }\end{array}$ & $\begin{array}{c}* / l . \\
\text { wreath// } \\
\text { cons } \Delta\end{array}$ & $\begin{array}{l}\text { RIC 219, } \\
\quad 41 \mathrm{a}\end{array}$ & G60-231 & \\
\hline 400 & Valentinian I & $367-375$ & $A_{3}$ & $\notin$ & 17 & 2.66 & 12 & $\begin{array}{c}\text { [DN VALEN] } \\
\text { TINI-ANVS PF } \\
\text { AVG }\end{array}$ & $\begin{array}{l}\text { [GLORIA] RO- } \\
\text { MAN[ORVM] }\end{array}$ & $S M N B$ & $\begin{array}{l}\text { RIC 252, } \\
\text { 9a }\end{array}$ & G60-247 & \\
\hline 401 & Valentinian I & $367-375$ & Æ 3 & $\notin$ & 17 & 3.44 & 1 & $\begin{array}{l}\text { DN VALENTI- } \\
\text { NI-ANVS PF } \\
\text { AVG }\end{array}$ & $\begin{array}{l}\text { SECVRITAS } \\
\text { REIPVBLICAE }\end{array}$ & SM AQ? & $\begin{array}{l}\text { RIC 92, } \\
12 \mathrm{a}\end{array}$ & G6o-19 & \\
\hline 402 & Valentinian I & $367-375$ & $\notin_{3} 3$ & $\notin$ & 18 & 1.58 & 1 & $\begin{array}{c}\text { [DN VALENTI- } \\
\text { NI-AN]VS PF } \\
\text { AVG }\end{array}$ & $\begin{array}{l}\text { [SEC]VRITAS } \\
\text { REIPVBLICAE }\end{array}$ & $\begin{array}{c}* /--/ / S M \\
\text { AQP }\end{array}$ & $\begin{array}{c}\text { RIC 96, } \\
12 \mathrm{a}\end{array}$ & G60-212 & \\
\hline 403 & Valentinian I & $367-375$ & $Æ_{3} 3$ & $\notin$ & 18 & 2.44 & 6 & $\begin{array}{l}\text { DN VALENTI- } \\
\text { NI-ANVS PF } \\
\text { AVG }\end{array}$ & $\begin{array}{l}\text { SECVRITAS } \\
\text { REIPVBLICAE }\end{array}$ & $\begin{array}{c}* /--/ / S M \\
\text { AQP P }\end{array}$ & $\begin{array}{l}\text { RIC 96, } \\
12 \mathrm{a}\end{array}$ & G60-248 & \\
\hline
\end{tabular}




\begin{tabular}{|c|c|c|c|c|c|c|c|c|c|c|c|c|c|}
\hline \multicolumn{14}{|c|}{ SOTIN } \\
\hline $\begin{array}{l}\text { CAT. } \\
\text { NO. }\end{array}$ & RULER & $\begin{array}{l}\text { DATING } \\
\text { (YRS) }\end{array}$ & DENOM. & MAT. & $\begin{array}{c}\text { D. } \\
(\mathrm{mm})\end{array}$ & $\begin{array}{l}\text { WT. } \\
(\mathrm{g})\end{array}$ & $\begin{array}{l}\text { ORI- } \\
\text { ENT. }\end{array}$ & OBVERSE & REVERSE & $\begin{array}{c}\text { MINT } \\
\text { MARKS }\end{array}$ & $\begin{array}{l}\text { REFE- } \\
\text { RENCE }\end{array}$ & $\begin{array}{l}\text { INV. } \\
\text { NO. }\end{array}$ & NOTE \\
\hline 404 & Valentinian I & $367-375$ & E 3 & E & 18 & 2.61 & 1 & $\begin{array}{c}\text { DN VALENTI- } \\
\text { NI-ANVS PF } \\
\text { AVG }\end{array}$ & $\begin{array}{l}\text { SECVRITAS } \\
\text { REIPVBLICAE }\end{array}$ & $\begin{array}{c}\text { R SECNVN- } \\
\text { DA }\end{array}$ & $\begin{array}{l}\text { RIC 121, } \\
24 \mathrm{a}\end{array}$ & C23123 & \\
\hline 405 & Valentinian I & $367-375$ & E 3 & E & 19 & 2.25 & 6 & $\begin{array}{c}\text { DN VALENTI- } \\
\text { NI-ANVS PF } \\
\text { AVG }\end{array}$ & $\begin{array}{l}\text { SECVRITAS } \\
\text { REIPVBLICAE }\end{array}$ & R TERTIA & $\begin{array}{l}\text { RIC 121, } \\
24 \mathrm{a}\end{array}$ & G60-161 & \\
\hline 406 & Valentinian I & $367-375$ & E 3 & E & 18 & 2.58 & 12 & $\begin{array}{c}\text { [DN V]ALEN- } \\
\text { TINI-ANVS PF } \\
\text { AVG }\end{array}$ & $\begin{array}{l}\text { SECVRITAS } \\
\text { REIPVBLICAE }\end{array}$ & SM leaf RP & $\begin{array}{l}\text { RIC 121, } \\
24 \mathrm{a}\end{array}$ & G60-271 & \\
\hline 407 & Valentinian I & $367-375$ & E 3 & E & 18 & 1.88 & 5 & $\begin{array}{c}\text { DN VALENTI- } \\
\text { NI-[AN]VS PF } \\
\text { AV[G] }\end{array}$ & $\begin{array}{l}\text { SECVRITAS } \\
\text { REIPVBLICAE }\end{array}$ & $\mathrm{R} \cdot[\mathrm{PR}] \mathrm{IMA}$ & $\begin{array}{l}\text { RIC 121, } \\
24 \mathrm{a}\end{array}$ & G61-48 & \\
\hline 408 & Valentinian I & $367-375$ & E 3 & E & 17 & 1.89 & 6 & $\begin{array}{c}\text { DN } \\
\text { VALENT[INI- } \\
\text { ANVS PF } \\
\text { AVG] }\end{array}$ & $\begin{array}{l}\text { [SECVRITAS } \\
\text { REIPVBLICAE] }\end{array}$ & {$[\mathrm{R}]$ TERTIA } & $\begin{array}{l}\text { RIC 121, } \\
24 \mathrm{C}\end{array}$ & G61-66 & \\
\hline 409 & Valentinian I & $367-375$ & E 3 & E & 17 & 2.37 & 7 & $\begin{array}{c}\text { [DN]VALEN- } \\
\text { TINI-ANVS PF } \\
\text { AVG }\end{array}$ & $\begin{array}{l}\text { SECVRITA[S] } \\
\text { REIPVBLICAE }\end{array}$ & $\begin{array}{c}\mathrm{D} /--/ / * \Delta \\
\text { SIS C }\end{array}$ & $\begin{array}{c}\text { RIC 147, } \\
15 a\end{array}$ & C23252 & \\
\hline 410 & Valentinian I & $367-375$ & E 3 & E & 18 & 2.24 & 12 & $\begin{array}{l}\text { DN VALENTI- } \\
\text { NI-ANVS PF } \\
\text { AVG }\end{array}$ & $\begin{array}{l}\text { SECVRITAS } \\
\text { REIPVBLICAE }\end{array}$ & $\begin{array}{l}* \mathrm{~K} / \mathrm{Q} / / \Gamma \\
\mathrm{SISCV}\end{array}$ & $\begin{array}{c}\text { RIC 147, } \\
15 a\end{array}$ & C23307 & \\
\hline 411 & Valentinian I & $367-375$ & Æ3 & E & 18 & 2.28 & 12 & $\begin{array}{c}\text { DN VALENTI- } \\
\text { NI-ANVS PF } \\
\text { AVG }\end{array}$ & $\begin{array}{l}\text { SECVRITAS } \\
\text { REIPVBLICAE }\end{array}$ & $\begin{array}{c}\mathrm{D} /--/ / * \mathrm{~A} \\
\text { SIS C }\end{array}$ & $\begin{array}{c}\text { RIC 147, } \\
15 a\end{array}$ & G60-22 & \\
\hline 412 & Valentinian I & $367-375$ & E 3 & E & 17 & 1.96 & 2 & $\begin{array}{c}\text { [DN VALE] } \\
\text { NTINI-ANVS } \\
\text { PF AVG }\end{array}$ & $\begin{array}{l}\text { [SECVRITAS] } \\
\text { REIPVBLICAE }\end{array}$ & $\begin{array}{c}* \mathrm{D} / \mathrm{S} / /[\mathrm{L}] \\
\mathrm{SISC}\end{array}$ & $\begin{array}{l}\text { RIC 147, } \\
15 \mathrm{a}\end{array}$ & G60-39 & \\
\hline 413 & Valentinian I & $367-375$ & $\mathbb{E}_{3}$ & E & 17 & 2.53 & 7 & $\begin{array}{c}\text { DN VALENTI- } \\
\text { NI-[ANVS] PF } \\
\text { AVG }\end{array}$ & $\begin{array}{c}\text { SECVRITAS } \\
\text { [REIPVBLICAE] }\end{array}$ & $\begin{array}{c}* \mathrm{~F} / \mathrm{M} / / \Delta \\
\text { SIS C }\end{array}$ & $\begin{array}{l}\text { RIC 147, } \\
15 a\end{array}$ & G60-44 & \\
\hline 414 & Valentinian I & $367-375$ & E 3 & $\mathbb{E}$ & 18 & 2.03 & 6 & $\begin{array}{l}\text { DN VALENTI- } \\
\text { NI-ANVS [PF] } \\
\text { AVG }\end{array}$ & $\begin{array}{c}\text { SECVRITAS } \\
\text { RE[IPVBLICAE] }\end{array}$ & $\begin{array}{l}\mathrm{CÁ} / \mathrm{S} \cdot / / \Gamma \\
\mathrm{SIS} C 4\end{array}$ & $\begin{array}{l}\text { RIC 147, } \\
15 a\end{array}$ & G60-50 & \\
\hline 415 & Valentinian I & $367-375$ & E 3 & $\mathbb{E}$ & 18 & 2.26 & 7 & $\begin{array}{c}\text { DN VALENTI- } \\
\text { NI-ANVS PF } \\
\text { AVG }\end{array}$ & $\begin{array}{l}\text { SECVRITAS } \\
\text { REIPVBLICAE }\end{array}$ & $\begin{array}{l}\mathrm{R} /--/ / \cdot A \\
\text { SIS C }\end{array}$ & $\begin{array}{l}\text { RIC 147, } \\
15 a\end{array}$ & G60-54 & \\
\hline 416 & Valentinian I & $367-375$ & E 3 & $\mathbb{E}$ & 18 & 1.87 & 6 & $\begin{array}{c}\text { DN VALENTI- } \\
\text { NI-ANVS PF } \\
\text { AVG }\end{array}$ & $\begin{array}{l}\text { SECVRIT[AS] } \\
\text { REIPVBLICAE }\end{array}$ & $\begin{array}{l}* F / M / / \Delta \\
\text { SIS C }\end{array}$ & $\begin{array}{c}\text { RIC 147, } \\
15 a\end{array}$ & G60-78 & \\
\hline 417 & Valentinian I & $367-375$ & E 3 & E & 18 & 2.41 & 1 & $\begin{array}{l}\text { DN VALENTI- } \\
\text { NI-ANVS PF } \\
\text { AVG }\end{array}$ & $\begin{array}{l}\text { SECVRITAS } \\
\text { REIPVBLICAE }\end{array}$ & $\begin{array}{l}\mathrm{R} /--/ / \cdot \mathrm{A} \\
\text { SIS C }\end{array}$ & $\begin{array}{l}\text { RIC 147, } \\
15 a\end{array}$ & G60-81 & \\
\hline 418 & Valentinian I & $367-375$ & E 3 & $\mathbb{E}$ & 17 & 2.44 & 6 & $\begin{array}{l}\text { DN VALENTI- } \\
\text { NI-ANVS PF } \\
\text { AVG }\end{array}$ & $\begin{array}{l}\text { SECVRITAS } \\
\text { REIPVBLICAE }\end{array}$ & $\begin{array}{c}* \mathrm{P} / \mathrm{M} / / \Delta \\
\mathrm{SISC}\end{array}$ & $\begin{array}{c}\text { RIC 147, } \\
15 a\end{array}$ & G60-84 & \\
\hline 419 & Valentinian I & $367-375$ & $E_{3}$ & $A$ & 18 & 3.1 & 7 & $\begin{array}{l}\text { DN VALENTI- } \\
\text { NI-ANVS PF } \\
\text { AVG }\end{array}$ & $\begin{array}{l}\text { SECVRITAS } \\
\text { REIPVBLICAE }\end{array}$ & $\begin{array}{c}\mathrm{D} /--/ / * \Delta \\
\text { SIS C }\end{array}$ & $\begin{array}{l}\text { RIC 147, } \\
15 a\end{array}$ & G60-86 & \\
\hline 420 & Valentinian I & $367-375$ & $\mathbb{E}_{3}$ & E & 18 & 2.26 & 5 & $\begin{array}{l}\text { DN VALENTI- } \\
\text { NI-ANVS PF } \\
\text { AVG }\end{array}$ & $\begin{array}{l}\text { SECVRITAS } \\
\text { REIPVBLICAE }\end{array}$ & $\begin{array}{c}* \mathrm{P} / \mathrm{M} / / \Gamma \\
\mathrm{SISC}\end{array}$ & $\begin{array}{l}\text { RIC 147, } \\
15 a\end{array}$ & G60-92 & \\
\hline 421 & Valentinian I & $367-375$ & E 3 & E & 19 & 2.25 & 5 & $\begin{array}{l}\text { DN VALENTI- } \\
\text { NI-ANVS PF } \\
\text { AVG }\end{array}$ & $\begin{array}{l}\text { SECVRIT[AS] } \\
\text { REIPVBLICAE }\end{array}$ & $\begin{array}{l}\mathrm{R} /--/ / \cdot A \\
\text { SIS C }\end{array}$ & $\begin{array}{l}\text { RIC 147, } \\
15 a\end{array}$ & G60-98 & \\
\hline 422 & Valentinian I & $367-375$ & E 3 & E & 18 & 2.59 & 1 & $\begin{array}{c}\text { DN VALENTI- } \\
\text { NI-AN[VS PF } \\
\text { AVG] }\end{array}$ & $\begin{array}{l}\text { [SECVR]ITAS } \\
\text { REIPVBLICAE }\end{array}$ & $\begin{array}{c}\mathrm{D} /--/ / * ? \\
\text { SIS C }\end{array}$ & $\begin{array}{l}\text { RIC 147, } \\
15 a\end{array}$ & G60-102 & \\
\hline 423 & Valentinian I & $367-375$ & E 3 & $\mathbb{E}$ & 18 & 1.55 & 6 & $\begin{array}{c}\text { DN VALENTI- } \\
\text { NI-ANVS PF } \\
\text { AVG }\end{array}$ & $\begin{array}{l}\text { SECVRITAS } \\
\text { REIPVBLICAE }\end{array}$ & $\begin{array}{c}* \mathrm{RO} / \mathrm{Q} / /[\mathrm{[}] \\
\mathrm{SISC} 4\end{array}$ & $\begin{array}{l}\text { RIC 147, } \\
15 a\end{array}$ & G60-109 & \\
\hline 424 & Valentinian I & $367-375$ & $E_{3}$ & E & 18 & 2.33 & 12 & $\begin{array}{l}\text { DN VALENTI- } \\
\text { NI-ANVS PF } \\
\text { AVG }\end{array}$ & $\begin{array}{l}\text { SECVRITAS } \\
\text { REIPVBLICAE }\end{array}$ & $\begin{array}{l}\mathrm{R} /--/ / \cdot \Delta \\
\text { SIS C }\end{array}$ & $\begin{array}{c}\text { RIC 147, } \\
15 a\end{array}$ & G60-114 & \\
\hline 425 & Valentinian I & $367-375$ & E 3 & E & 19 & 2 & 1 & $\begin{array}{c}\text { DN VALENTI- } \\
\text { NI-ANVS PF } \\
\text { AVG }\end{array}$ & $\begin{array}{l}\text { SECVRI[TAS } \\
\text { REIPVBLICAE] }\end{array}$ & $\begin{array}{l}\mathrm{R} /--/ / \cdot A \\
\text { SIS C }\end{array}$ & $\begin{array}{l}\text { RIC 147, } \\
15 a\end{array}$ & G60-115 & \\
\hline
\end{tabular}




\begin{tabular}{|c|c|c|c|c|c|c|c|c|c|c|c|c|c|}
\hline \multicolumn{14}{|c|}{ SOTIN } \\
\hline $\begin{array}{l}\text { CAT. } \\
\text { NO. }\end{array}$ & RULER & $\begin{array}{l}\text { DATING } \\
\text { (YRS) }\end{array}$ & DENOM. & MAT. & $\begin{array}{c}\mathrm{D} . \\
(\mathrm{mm})\end{array}$ & $\begin{array}{l}\text { WT. } \\
\text { (g) }\end{array}$ & $\begin{array}{l}\text { ORI- } \\
\text { ENT. }\end{array}$ & OBVERSE & REVERSE & $\begin{array}{l}\text { MINT } \\
\text { MARKS }\end{array}$ & $\begin{array}{l}\text { REFE- } \\
\text { RENCE }\end{array}$ & $\begin{array}{l}\text { INV. } \\
\text { NO. }\end{array}$ & NOTE \\
\hline 426 & Valentinian I & $367-375$ & Æ 3 & $\notin$ & 18 & 2.63 & 6 & $\begin{array}{l}\text { DN VALENTI- } \\
\text { NI-ANVS PF } \\
\text { AVG }\end{array}$ & $\begin{array}{l}\text { SECVRITAS } \\
\text { REIPVBLICAE }\end{array}$ & $\begin{array}{l}* \mathrm{P} / \mathrm{M} / / \Delta \\
\mathrm{SISC}\end{array}$ & $\begin{array}{l}\text { RIC 147, } \\
15 \mathrm{a}\end{array}$ & G60-121 & \\
\hline 427 & Valentinian I & $367-375$ & $\notin_{3} 3$ & $\notin$ & 18 & 1.82 & 12 & $\begin{array}{l}\text { DN VALENTI- } \\
\text { NI-ANVS PF } \\
\text { AVG }\end{array}$ & $\begin{array}{l}\text { SECVRITAS } \\
\text { REIPVBLICAE }\end{array}$ & $\begin{array}{l}\text { D/--//·A } \\
\text { SIS C }\end{array}$ & $\begin{array}{l}\text { RIC 147, } \\
15 \mathrm{a}\end{array}$ & G60-122 & \\
\hline 428 & Valentinian I & $367-375$ & $\mathbb{E}_{3}$ & $\notin$ & 17 & 2.46 & 6 & $\begin{array}{l}\text { DN VALENTI- } \\
\text { NI-ANVS PF } \\
\text { AVG }\end{array}$ & $\begin{array}{l}\text { SECVRITAS } \\
\text { REIPVBLICAE }\end{array}$ & $\begin{array}{l}\mathrm{D} /--/ / * \Delta \\
\text { SIS C }\end{array}$ & $\begin{array}{l}\text { RIC 147, } \\
15 \mathrm{a}\end{array}$ & G60-127 & \\
\hline 429 & Valentinian I & $367-375$ & $A_{3}$ & $\notin$ & 18 & 1.56 & 1 & $\begin{array}{c}\text { DN VALENTI- } \\
\text { NI-[ANVS PF } \\
\text { AVG] }\end{array}$ & $\begin{array}{l}\text { [SECVRITA]S } \\
\text { REIPVBLICAE }\end{array}$ & $\begin{array}{l}\mathrm{RÁ} / \mathrm{S} \cdot / / \Gamma \\
\mathrm{SISC} C\end{array}$ & $\begin{array}{l}\text { RIC 147, } \\
15 \mathrm{a}\end{array}$ & G60-145 & \\
\hline 430 & Valentinian I & $367-375$ & $\mathbb{E}_{3}$ & $\notin$ & 18 & 2 & 7 & $\begin{array}{l}\text { DN VALENTI- } \\
\text { NI-ANVS PF } \\
\text { AVG }\end{array}$ & $\begin{array}{l}\text { SECVRITAS } \\
\text { REIPVB[LICAE] }\end{array}$ & $\begin{array}{l}* \mathrm{~K} / \mathrm{Q} / / \Gamma \\
\mathrm{SISCV}\end{array}$ & $\begin{array}{l}\text { RIC 147, } \\
15 \mathrm{a}\end{array}$ & G60-158 & \\
\hline 431 & Valentinian I & $367-375$ & $Æ_{3}$ & $\notin$ & 17 & 2.37 & 12 & $\begin{array}{c}\text { DN } \\
\text { VALEN[TINI- } \\
\text { A]NVS PF } \\
\text { AVG }\end{array}$ & $\begin{array}{l}\text { SECVRITAS } \\
\text { REIPVBL[ICAE] }\end{array}$ & $\begin{array}{l}\mathrm{D} /--/ / * \Delta \\
\text { SIS C }\end{array}$ & $\begin{array}{l}\text { RIC 147, } \\
15 \mathrm{a}\end{array}$ & G60-178 & \\
\hline 432 & Valentinian I & $367-375$ & $\notin_{3}$ & $E$ & 19 & 2.62 & 1 & $\begin{array}{c}\text { DN } \\
\text { VALE[NTINI- } \\
\text { A]NVS[PF] } \\
\text { AVG }\end{array}$ & $\begin{array}{l}\text { [SECVRITAS REI] } \\
\text { PVBL[ICAE] }\end{array}$ & $\begin{array}{l}\mathrm{D} /--/ / ? \\
\mathrm{SISC}\end{array}$ & $\begin{array}{l}\text { RIC 147, } \\
15 \mathrm{a}\end{array}$ & G60-196 & \\
\hline 433 & Valentinian I & $367-375$ & $A_{3} 3$ & $\notin$ & 9 & 2.49 & 6 & $\begin{array}{l}\text { [DN V]ALEN- } \\
\text { TINI-ANVS PF } \\
\text { AVG }\end{array}$ & $\begin{array}{l}\text { SECVRITAS } \\
\text { REIPVBLICAE }\end{array}$ & $\begin{array}{l}* \Gamma \mathrm{\Gamma K} / \mathrm{Q} / / \\
\mathrm{SISCV}\end{array}$ & $\begin{array}{l}\text { RIC 147, } \\
15 \mathrm{a}\end{array}$ & G60-207 & \\
\hline 434 & Valentinian I & $367-375$ & $Æ_{3} 3$ & $\notin$ & 17 & 1.88 & 7 & $\begin{array}{c}\text { [DN VALENTI] } \\
\text { NI-ANVS P[F } \\
\text { AVG] }\end{array}$ & $\begin{array}{l}\text { SECVRITAS } \\
\text { REIP[VBLICAE] }\end{array}$ & $\begin{array}{c}\mathrm{R} /--/ /[\cdot \mathrm{A} \\
\mathrm{SISC}]\end{array}$ & $\begin{array}{l}\text { RIC 147, } \\
15 \mathrm{a}\end{array}$ & G60-227 & \\
\hline 435 & Valentinian I & $367-375$ & $\notin_{3} 3$ & $\notin$ & 18 & 2.34 & 6 & $\begin{array}{l}\text { DN VALENTI- } \\
\text { NI-ANVS PF } \\
\text { AVG }\end{array}$ & $\begin{array}{c}\text { SECVRITAS } \\
\text { REIPVBLICAE }\end{array}$ & $\begin{array}{l}\text { RÁ/S } / / / \Gamma \\
\text { SIS C K }\end{array}$ & $\begin{array}{c}\text { RIC 147, } \\
15 \mathrm{a}\end{array}$ & G60-268 & \\
\hline 436 & Valentinian I & $367-375$ & $Æ_{3} 3$ & $\notin$ & 17 & 2.58 & 1 & $\begin{array}{c}\text { [DN } \\
\text { VALENTINI]- } \\
\text { ANVS PF AVG }\end{array}$ & $\begin{array}{l}\text { [SECVRITAS] } \\
\text { REIPVBLICAE }\end{array}$ & $\begin{array}{l}\mathrm{D} / \mathrm{S} / / \Delta \\
\mathrm{SISC}\end{array}$ & $\begin{array}{l}\text { RIC 147, } \\
15 \mathrm{a}\end{array}$ & G60-269 & \\
\hline 437 & Valentinian I & $367-375$ & $A_{3} 3$ & $E$ & 18 & 2.35 & 12 & $\begin{array}{c}\text { DN } \\
\text { VALEN[TINI- } \\
\text { A]NVS PF } \\
\text { AVG }\end{array}$ & $\begin{array}{l}\text { SECVRIT[AS RE] } \\
\text { IPVBLICAE }\end{array}$ & $\begin{array}{l}\text { D/--//*A } \\
\text { SIS C }\end{array}$ & $\begin{array}{l}\text { RIC 147, } \\
15 \mathrm{a}\end{array}$ & G60-273 & \\
\hline 438 & Valentinian I & $367-375$ & $\notin_{3}$ & $\notin$ & 17 & 2.23 & 6 & $\begin{array}{c}\text { [DN } \\
\text { VALENTINI]- } \\
\text { ANVS PF AVG }\end{array}$ & $\begin{array}{c}\text { [SECVRITAS REIP] } \\
\text { VBLICAE }\end{array}$ & $\begin{array}{l}\mathrm{R} /--/ / \cdot \mathrm{A} \\
\mathrm{SISC}\end{array}$ & $\begin{array}{l}\text { RIC 147, } \\
15 \mathrm{a}\end{array}$ & G60-283 & \\
\hline 439 & Valentinian I & $367-375$ & E 3 & $E$ & 18 & 2.32 & 7 & $\begin{array}{l}\text { DN VALENTI- } \\
\text { NI-ANVS PF } \\
\text { AVG }\end{array}$ & $\begin{array}{l}\text { SECVRITAS } \\
\text { REIPVBLICAE }\end{array}$ & $\begin{array}{l}\text { R/--I/PA } \\
\text { SIS C }\end{array}$ & $\begin{array}{l}\text { RIC 147, } \\
15 \mathrm{a}\end{array}$ & G61-3 & \\
\hline 440 & Valentinian I & $367-375$ & Æ 3 & 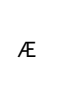 & 16 & 2.33 & 5 & $\begin{array}{l}\text { DN VALENTI- } \\
\text { NI-ANVS PF } \\
\text { AVG }\end{array}$ & $\begin{array}{l}\text { SECVRITAS } \\
\text { REIPVBLICAE }\end{array}$ & $\begin{array}{l}* \mathrm{RO} / \mathrm{Q} / / \Gamma \\
\mathrm{SISC} 4\end{array}$ & $\begin{array}{l}\text { RIC 147, } \\
15 \mathrm{a}\end{array}$ & G61-14 & \\
\hline 441 & Valentinian I & $367-375$ & $\notin_{3} 3$ & $\notin$ & 18 & 2.99 & 2 & $\begin{array}{l}\text { DN VALENTI- } \\
\text { NI-ANVS PF } \\
\text { AVG }\end{array}$ & $\begin{array}{l}\text { SECVRITAS } \\
\text { REIPVBLICAE }\end{array}$ & $\begin{array}{l}\text { R/--I/PA } \\
\text { SIS C }\end{array}$ & $\begin{array}{l}\text { RIC 147, } \\
15 \mathrm{a}\end{array}$ & G61-18 & \\
\hline 442 & Valentinian I & $367-375$ & $\notin_{3}$ & E & 17 & 2.2 & 2 & $\begin{array}{l}\text { DN VALENTI- } \\
\text { NI-[ANVS PF } \\
\text { AVG] }\end{array}$ & $\begin{array}{l}\text { SECVRITAS } \\
\text { REIPVBLICAE }\end{array}$ & $\begin{array}{l}\mathrm{D} / \mathrm{S} / / \Delta \\
\mathrm{SISC}\end{array}$ & $\begin{array}{c}\text { RIC 147, } \\
15 \mathrm{a}\end{array}$ & G61-19 & \\
\hline 443 & Valentinian I & $367-375$ & $A_{3}$ & $A$ & 18 & 2.3 & 1 & $\begin{array}{l}\text { DN VALENTI- } \\
\text { NI-ANVS PF } \\
\text { AVG }\end{array}$ & $\begin{array}{l}\text { SECVRITAS } \\
\text { REIPVBLICAE }\end{array}$ & $\begin{array}{l}\text { R/--I/PA } \\
\text { SIS C }\end{array}$ & $\begin{array}{l}\text { RIC 147, } \\
15 \mathrm{a}\end{array}$ & G61-45 & \\
\hline 444 & Valentinian I & $367-375$ & $A_{3}$ & E & 17 & 2.2 & 6 & $\begin{array}{c}\text { DN } \\
\text { V[ALENTINI- } \\
\text { AN]VS PF } \\
\text { AVG }\end{array}$ & $\begin{array}{c}\text { SECVRITAS } \\
\text { [REIPVBLICAE] }\end{array}$ & $\begin{array}{l}* \mathrm{P} / \mathrm{M} / / \Delta \\
\mathrm{SISC}\end{array}$ & $\begin{array}{l}\text { RIC 147, } \\
15 \mathrm{a}\end{array}$ & G61-46 & \\
\hline 445 & Valentinian I & $367-375$ & $\notin_{3}$ & $\notin$ & 17 & 1.91 & 7 & $\begin{array}{c}\text { DN } \\
\text { VALE[NTINI]- } \\
\text { ANVS PF AVG }\end{array}$ & $\begin{array}{l}\text { SECVRITAS } \\
\text { REIPVBLICAE }\end{array}$ & $\begin{array}{c}* \mathrm{P} / \mathrm{M} / / \text { ?SIS } \\
\mathrm{C}\end{array}$ & $\begin{array}{l}\text { RIC 147, } \\
15 \mathrm{a}\end{array}$ & G61-55 & \\
\hline 446 & Valentinian I & $367-375$ & $A_{3}$ & $\notin$ & 17 & 2.31 & 6 & $\begin{array}{l}\text { DN VALENTI- } \\
\text { NI-ANVS PF } \\
\text { AVG }\end{array}$ & $\begin{array}{l}\text { SECVRITAS } \\
\text { REIPVBLICAE }\end{array}$ & $\begin{array}{c}* \mathrm{P} / \mathrm{M} / / \Gamma \\
\mathrm{SISC}\end{array}$ & $\begin{array}{l}\text { RIC 147, } \\
15 \mathrm{a}\end{array}$ & G67-1 & \\
\hline
\end{tabular}




\begin{tabular}{|c|c|c|c|c|c|c|c|c|c|c|c|c|c|}
\hline \multicolumn{14}{|c|}{ SOTIN } \\
\hline $\begin{array}{l}\text { CAT. } \\
\text { NO. }\end{array}$ & RULER & $\begin{array}{l}\text { DATING } \\
\text { (YRS) }\end{array}$ & DENOM. & MAT. & $\begin{array}{c}\text { D. } \\
(\mathrm{mm})\end{array}$ & $\begin{array}{l}\text { WT. } \\
\text { (g) }\end{array}$ & $\begin{array}{l}\text { ORI- } \\
\text { ENT. }\end{array}$ & OBVERSE & REVERSE & $\begin{array}{l}\text { MINT } \\
\text { MARKS }\end{array}$ & $\begin{array}{l}\text { REFE- } \\
\text { RENCE }\end{array}$ & $\begin{array}{l}\text { INV. } \\
\text { NO. }\end{array}$ & NOTE \\
\hline 447 & Valentinian I & $367-375$ & $Æ_{3}$ & $E$ & 18 & 1.63 & 6 & $\begin{array}{c}\text { DN VALENTI- } \\
\text { NI-ANVS[PF } \\
\text { AVG] }\end{array}$ & $\begin{array}{c}\text { SECVRITAS } \\
\text { [REIPVBLICAE] }\end{array}$ & $\begin{array}{l}\mathrm{D} /--/ / ? \Delta \\
\text { SIS C }\end{array}$ & $\begin{array}{c}\text { RIC 147 } \\
15 a\end{array}$ & G69-1 & \\
\hline 448 & Valentinian I & $367-375$ & $\notin 3$ & EE & 17 & 2.59 & 5 & $\begin{array}{c}\text { DN VALENTI- } \\
\text { NI-ANVS PF } \\
\text { AVG }\end{array}$ & $\begin{array}{l}\text { SECVRITAS } \\
\text { REIPVBLICAE }\end{array}$ & A/--//TES & $\begin{array}{c}\text { RIC 178 } \\
27 a\end{array}$ & G60-55 & \\
\hline 449 & Valentinian I & $367-375$ & Æ 3 & E & 17 & 2.19 & 5 & \begin{tabular}{|c|} 
DN \\
VALEN[TINI-] \\
ANVS P[F \\
AVG]
\end{tabular} & $\begin{array}{l}\text { [SECVRITAS] } \\
\text { REIPVBLICAE }\end{array}$ & $* \Gamma / * / /$ TES & $\begin{array}{l}\text { RIC 178 } \\
27 a\end{array}$ & G60-64 & \\
\hline 450 & Valentinian I & $367-375$ & $Æ_{3}$ & EE & 18 & 2.29 & 5 & $\begin{array}{c}\text { DN VALENTI- } \\
\text { NI-ANVS PF } \\
\text { AVG }\end{array}$ & $\begin{array}{l}\text { SECVRITAS } \\
\text { REIPVBLICAE }\end{array}$ & V/Г//[TES] & $\begin{array}{l}\text { RIC 178 } \\
27 a\end{array}$ & G60-159 & \\
\hline 451 & $\begin{array}{l}\text { Valent. I/ } \\
\text { Valens }\end{array}$ & $367-375$ & $Æ_{3} 3$ & E & 17 & 1.54 & 7 & DN VALE? & $\begin{array}{c}\text { SECVRITAS } \\
\text { [REIPV]BLI[CAE] }\end{array}$ & $S M A Q P$ & $\begin{array}{l}\text { RIC 96, } \\
12 a-b\end{array}$ & G61-56 & \\
\hline 452 & Valens & $367-375$ & Æ 3 & E & 17 & 1.54 & 1 & $\begin{array}{l}\text { DN VALEN-S } \\
\text { PF AV[G] }\end{array}$ & $\begin{array}{l}\text { GLORIA RO-MA- } \\
\text { NORVM }\end{array}$ & $\begin{array}{c}--/ * / / S M \\
A Q P \\
\end{array}$ & $\begin{array}{c}\text { RIC } 96 \\
11 b\end{array}$ & C23449 & \\
\hline 453 & Valens & $367-375$ & Æ 3 & $\notin$ & 18 & 2.25 & 12 & $\begin{array}{c}\text { DN VALEN-S } \\
\text { PF AVG }\end{array}$ & $\begin{array}{l}\text { GLORIA RO-MA- } \\
\text { NORVM }\end{array}$ & $S M A[Q] P$ & $\begin{array}{l}\text { RIC 96, } \\
11 \mathrm{~b}\end{array}$ & G67-12 & \\
\hline 454 & Valens & $367-375$ & Æ 3 & E & 16 & 2.26 & 2 & $\begin{array}{c}\text { [DN V]ALEN-S } \\
\text { PF AVG }\end{array}$ & $\begin{array}{c}\text { GLORIA RO-MA- } \\
\text { NORVM }\end{array}$ & $\begin{array}{l}--/ R / / \cdot B \\
\text { SIS C }\end{array}$ & $\begin{array}{l}\text { RIC 147, } \\
14 \mathrm{~b}\end{array}$ & G60-25 & \\
\hline 455 & Valens & $367-375$ & Æ3 & AE & 17 & 1.96 & 6 & $\begin{array}{c}\text { DN VALEN-[S } \\
\text { PF AVG] }\end{array}$ & $\begin{array}{l}\text { GLORIA RO-MA- } \\
\text { NORVM }\end{array}$ & $\begin{array}{l}--/ D / / * \Gamma \\
\text { SIS C }\end{array}$ & $\begin{array}{l}\text { RIC 147, } \\
14 \mathrm{~b}\end{array}$ & G60-73 & \\
\hline 456 & Valens & $367-375$ & Æ 3 & $\mathscr{E}$ & 18 & 2.73 & 7 & $\begin{array}{l}\text { DN VALEN-S } \\
\text { PF AVG }\end{array}$ & $\begin{array}{c}\text { GLORIA RO-MA- } \\
\text { NORVM }\end{array}$ & $\begin{array}{l}--/ R / / \cdot \Gamma \\
\text { SIS C }\end{array}$ & $\begin{array}{l}\text { RIC 147, } \\
14 \mathrm{~b}\end{array}$ & G60-76 & \\
\hline 457 & Valens & $367-375$ & $Æ_{3}$ & 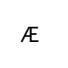 & 17 & 2.34 & 7 & $\begin{array}{c}\text { DN VALEN-[S } \\
\text { P]F AVG }\end{array}$ & $\begin{array}{l}\text { GLORIA RO- } \\
\text { [MANORVM] }\end{array}$ & $\begin{array}{l}\mathrm{R} /--/ / \cdot \Gamma \\
\mathrm{SISC}\end{array}$ & $\begin{array}{l}\text { RIC 147, } \\
14 \mathrm{~b}\end{array}$ & G60-117 & \\
\hline 458 & Valens & $367-375$ & Æ3 & $F$ & 17 & 2.31 & 7 & $\begin{array}{l}\text { DN VALEN-S } \\
\text { PF AVG }\end{array}$ & $\begin{array}{l}\text { [GL]ORIA RO- } \\
\text { MANORVM }\end{array}$ & $\begin{array}{l}--/ R / / \cdot \Gamma \\
\text { SIS C }\end{array}$ & $\begin{array}{l}\text { RIC 147, } \\
14 \mathrm{~b}\end{array}$ & G60-118 & \\
\hline 459 & Valens & $367-375$ & $Æ_{3} 3$ & 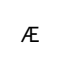 & 17 & 2.35 & 1 & $\begin{array}{c}\text { [DN VALEN]-S } \\
\text { PF AVG }\end{array}$ & $\begin{array}{l}\text { GLORIA RO-MA- } \\
\text { NORVM }\end{array}$ & $\begin{array}{l}--/ R / / \cdot B \\
\text { SIS C }\end{array}$ & $\begin{array}{l}\text { RIC 147, } \\
14 \mathrm{~b}\end{array}$ & G60-120 & \\
\hline 460 & Valens & $367-375$ & Æ 3 & $F$ & 17 & 2.02 & 2 & $\begin{array}{l}\text { DN VALEN-S } \\
\text { PF AVG }\end{array}$ & $\begin{array}{l}\text { GLORIA RO- } \\
\text { M[ANORVM] }\end{array}$ & $\begin{array}{l}--/ R / / \cdot \Gamma \\
\text { SIS C }\end{array}$ & $\begin{array}{l}\text { RIC 147, } \\
14 \mathrm{~b}\end{array}$ & G60-125 & \\
\hline 461 & Valens & $367-375$ & $Æ 3$ & 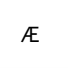 & 18 & 2.03 & 1 & $\begin{array}{l}\text { DN VALEN-S } \\
\text { PF AV[G] }\end{array}$ & $\begin{array}{l}\text { GLORIA RO- } \\
\text { [MANORVM] }\end{array}$ & $\begin{array}{l}--/ D / / *[\Gamma \\
\text { SIS C }]\end{array}$ & $\begin{array}{l}\text { RIC 147, } \\
14 \mathrm{~b}\end{array}$ & G60-191 & \\
\hline 462 & Valens & $367-375$ & Æ 3 & $Æ E$ & 17 & 2.52 & 1 & $\begin{array}{l}\text { DN VALEN-S } \\
\text { PF AVG }\end{array}$ & $\begin{array}{l}\text { GL[ORIA RO]- } \\
\text { MANORVM }\end{array}$ & $\begin{array}{l}--D / / * \Gamma \\
\text { SIS C }\end{array}$ & $\begin{array}{l}\text { RIC 147, } \\
14 \mathrm{~b}\end{array}$ & G61-8 & \\
\hline 463 & Valens & $367-375$ & $Æ 3$ & $Æ$ & 17 & 2.83 & 1 & $\begin{array}{l}\text { DN VALEN-S } \\
\text { PF A[VG] }\end{array}$ & $\begin{array}{l}\text { [GL]ORIA RO- } \\
\text { MANORVM }\end{array}$ & $\begin{array}{c}--/ D / / * \Gamma \\
\text { SIS C }\end{array}$ & $\begin{array}{l}\text { RIC 147, } \\
14 \mathrm{~b}\end{array}$ & G61-15 & \\
\hline 464 & Valens & $367-375$ & Æ 3 & $Æ$ & 19 & 1.97 & 1 & $\begin{array}{l}\text { DN VALEN-S } \\
\text { PF AVG }\end{array}$ & $\begin{array}{l}\text { [GLORIA R]O- } \\
\text { MANORVM }\end{array}$ & $\begin{array}{c}--/ R / / \cdot B \\
\text { SIS C }\end{array}$ & $\begin{array}{l}\text { RIC 147, } \\
14 \mathrm{~b}\end{array}$ & G61-36 & \\
\hline 465 & Valens & $367-375$ & E3 & $Æ$ & 20 & 1.8 & 12 & $\begin{array}{l}\text { DN VALEN-S } \\
\text { PF AVG }\end{array}$ & $\begin{array}{l}\text { GLORIA RO- } \\
\text { MANO[RVM] }\end{array}$ & $\begin{array}{l}--/ R / / \cdot B \\
\text { SIS C }\end{array}$ & $\begin{array}{l}\text { RIC 147, } \\
14 \mathrm{~b}\end{array}$ & G67-7 & \\
\hline 466 & Valens & $367-375$ & $Æ_{3}$ & E & 18 & 2.38 & 12 & $\begin{array}{l}\text { DN VALEN-S } \\
\text { PF AVG }\end{array}$ & $\begin{array}{l}\text { GLORIA RO- } \\
\text { MANO[RVM] }\end{array}$ & $\begin{array}{c}\text { I. } \\
\text { wreath//r// } \\
\text { TES }\end{array}$ & $\begin{array}{l}\text { RIC 178, } \\
26 \mathrm{~b}\end{array}$ & $\mathrm{C} 23680$ & \\
\hline 467 & Valens & $367-375$ & $Æ_{3}$ & E & 19 & 2.57 & 5 & $\begin{array}{l}\text { DN VALEN-S } \\
\text { PF AVG }\end{array}$ & $\begin{array}{c}\text { GLORIA RO-MA- } \\
\text { NORVM }\end{array}$ & $\mathrm{V} / * \mathrm{~A} / / \mathrm{TES}$ & $\begin{array}{l}\text { RIC } 178 \\
26 \mathrm{~b}\end{array}$ & $\mathrm{C} 23697$ & \\
\hline 468 & Valens & $367-375$ & Æ 3 & $Æ$ & 19 & 2.28 & 5 & $\begin{array}{l}\text { DN VALEN-S } \\
\text { PF AVG }\end{array}$ & $\begin{array}{l}\text { GLORIA RO-MA- } \\
\text { NORVM }\end{array}$ & $--/ * \mathrm{~A} / / \mathrm{TES}$ & $\begin{array}{l}\text { RIC } 178 \\
26 \mathrm{~b}\end{array}$ & G60-108 & \\
\hline 469 & Valens & $367-375$ & $\notin_{3} 3$ & $\notin$ & 18 & 2.61 & 6 & $\begin{array}{l}\text { DN VALEN-S } \\
\text { PF AVG }\end{array}$ & $\begin{array}{c}\text { GLORIA RO-[M] } \\
\text { ANORVM }\end{array}$ & $--/ \mathrm{B} / / \mathrm{TES}$ & $\begin{array}{l}\text { RIC } 178 \\
26 \mathrm{~b}\end{array}$ & G60-152 & \\
\hline 470 & Valens & $367-375$ & $\notin_{3} 3$ & EE & 18 & 2.03 & 12 & $\begin{array}{l}\text { DN VALEN-S } \\
\text { PF AVG }\end{array}$ & $\begin{array}{l}\text { GLORIA RO-MA- } \\
\text { NORVM }\end{array}$ & $--/ \mathrm{B} / / \cdot \mathrm{TES}$ & $\begin{array}{l}\text { RIC } 178 \\
26 \mathrm{~b}\end{array}$ & G60-202 & \\
\hline 471 & Valens & $367-375$ & $Æ_{3} 3$ & EE & 18 & 2.52 & 12 & $\begin{array}{l}\text { DN VALEN-S } \\
\text { PF AVG }\end{array}$ & $\begin{array}{l}\text { GLORIA RO- } \\
\text { [MAN]ORVM }\end{array}$ & $\mathrm{Z} / \mathrm{A} / / \mathrm{TES}$ & $\begin{array}{l}\text { RIC } 178 \\
26 \mathrm{~b}\end{array}$ & G60-245 & \\
\hline 472 & Valens & $367-375$ & Æ 3 & EE & 18 & 2.17 & 11 & $\begin{array}{l}\text { DN VALEN-S } \\
\text { PF AVG }\end{array}$ & $\begin{array}{l}\text { [GLORIA] RO- } \\
\text { MANORVM }\end{array}$ & $\begin{array}{l}--/ \Delta / /[\cdot] \\
\text { TES }\end{array}$ & $\begin{array}{l}\text { RIC } 178 \\
26 \mathrm{~b}\end{array}$ & G60-249 & \\
\hline 473 & Valens & $367-375$ & $Æ_{3}$ & EE & 17 & 1.96 & 11 & $\begin{array}{l}\text { DN VALEN-S } \\
\text { PF AVG }\end{array}$ & $\begin{array}{l}\text { GLORIA RO- } \\
\text { [MANORVM] }\end{array}$ & $\begin{array}{c}--/ \cdot \Delta / /[\cdot] \\
\text { TES }\end{array}$ & $\begin{array}{l}\text { RIC } 178 \\
26 \mathrm{~b}\end{array}$ & G60-250 & \\
\hline 474 & Valens & $367-375$ & $Æ_{3}$ & EE & 19 & 2.44 & 11 & $\begin{array}{c}\text { [DN VALEN-S } \\
\text { PF AVG] }\end{array}$ & $\begin{array}{l}\text { [GLORIA RO- } \\
\text { MANORVM] }\end{array}$ & $\begin{array}{c}--/ \cdot B / /[\cdot \\
T E S]\end{array}$ & $\begin{array}{l}\text { RIC } 178 \\
26 \mathrm{~b}\end{array}$ & G60-254 & \\
\hline 475 & Valens & $367-375$ & solidus & AV & & & & $\begin{array}{l}\text { DN VALENS } \\
\text { PER F AVG }\end{array}$ & $\begin{array}{l}\text { GLORIA R-O- } \\
\text { MANORVM }\end{array}$ & AN OB H & $\begin{array}{l}\text { RIC } 9276 \\
16 \mathrm{~d}\end{array}$ & C26228 & $\begin{array}{c}\text { Demo } \\
\text { (Ed.) } \\
1994\end{array}$ \\
\hline
\end{tabular}




\begin{tabular}{|c|c|c|c|c|c|c|c|c|c|c|c|c|c|}
\hline \multicolumn{14}{|c|}{ SOTIN } \\
\hline $\begin{array}{l}\text { CAT. } \\
\text { NO. }\end{array}$ & RULER & $\begin{array}{l}\text { DATING } \\
\text { (YRS) }\end{array}$ & DENOM. & MAT. & $\begin{array}{c}\text { D. } \\
(\mathrm{mm})\end{array}$ & $\begin{array}{l}\text { WT. } \\
\text { (g) }\end{array}$ & $\begin{array}{l}\text { ORI- } \\
\text { ENT. }\end{array}$ & OBVERSE & REVERSE & $\begin{array}{c}\text { MINT } \\
\text { MARKS }\end{array}$ & $\begin{array}{l}\text { REFE- } \\
\text { RENCE }\end{array}$ & $\begin{array}{l}\text { INV. } \\
\text { NO. }\end{array}$ & NOTE \\
\hline 476 & Valens & $367-375$ & E 3 & FE & 18 & 2.19 & 5 & $\begin{array}{l}\text { DN VALEN-S } \\
\text { PF AVG }\end{array}$ & $\begin{array}{l}\text { SECVRITAS } \\
\text { REIPVBLICAE }\end{array}$ & $\begin{array}{c}\mathrm{OF} / \mathrm{I} / / \mathrm{LVG} \\
\mathrm{PA}\end{array}$ & RIC $46,21 \mathrm{a}$ & G67-2 & \\
\hline 477 & Valens & $367-375$ & E 3 & $\mathscr{E}$ & 18 & 2 & 6 & $\begin{array}{l}\text { DN VALEN-S } \\
\text { PF AVG }\end{array}$ & $\begin{array}{l}\text { SECVRITAS } \\
\text { REIPVBLICAE }\end{array}$ & SM AQS & $\begin{array}{l}\text { RIC 96, } \\
12 \mathrm{~b}\end{array}$ & G6o-10 & \\
\hline 478 & Valens & $367-375$ & A 3 & $\mathscr{E}$ & 19 & 2.32 & 6 & $\begin{array}{l}\text { DN VALEN-S } \\
\text { PF AVG }\end{array}$ & $\begin{array}{l}\text { SECVRITAS } \\
\text { REIPVBLICAE }\end{array}$ & $S M A Q S$ & $\begin{array}{l}\text { RIC 96, } \\
12 \mathrm{~b}\end{array}$ & G60-61 & \\
\hline 479 & Valens & $367-375$ & E 3 & $\mathscr{E}$ & 18 & 1.8 & 7 & $\begin{array}{c}\text { [DN VA]LEN-S } \\
\text { PF AVG }\end{array}$ & $\begin{array}{l}\text { [SECVRITAS ] } \\
\text { REIPVBLICAE }\end{array}$ & $\cdot \mathrm{SM} A Q \mathrm{~S}$ & $\begin{array}{l}\text { RIC 96, } \\
12 \mathrm{~b}\end{array}$ & G60-180 & \\
\hline 480 & Valens & $367-375$ & E 3 & F & 19 & 2.74 & 4 & $\begin{array}{l}\text { DN VALEN-S } \\
\text { PF AVG }\end{array}$ & $\begin{array}{l}\text { [SECVRITAS } \\
\text { REIPVBLI]CAE }\end{array}$ & $\begin{array}{c}* /--/ / S M \\
\text { AQS }\end{array}$ & $\begin{array}{l}\text { RIC 96, } \\
12 \mathrm{~b}\end{array}$ & G60-244 & \\
\hline 481 & Valens & $367-375$ & E 3 & FE & 17 & 2.16 & 12 & $\begin{array}{c}\text { [DN VALEN]-S } \\
\text { PF AV[G] }\end{array}$ & $\begin{array}{l}\text { [SECV]RITAS } \\
\text { REI[PVBLICAE] }\end{array}$ & $\begin{array}{c}\text { I. wre- } \\
\text { ath/--//SM } \\
\text { AQS }\end{array}$ & $\begin{array}{l}\text { RIC 96, } \\
12 \mathrm{~b}\end{array}$ & G60-262 & \\
\hline 482 & Valens & $367-375$ & Æ3 & 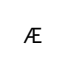 & 18 & 1.84 & 7 & $\begin{array}{c}\text { DN VALE[N]-S } \\
\text { PF AVG }\end{array}$ & $\begin{array}{c}\text { SECVRITAS } \\
\text { REIPV[BLICAE] }\end{array}$ & $\begin{array}{c}\mathrm{R} \cdot \mathrm{SECVN}- \\
\mathrm{DA}\end{array}$ & $\begin{array}{c}\text { RIC } 121 \\
24 \mathrm{~b}\end{array}$ & C23895 & \\
\hline 483 & Valens & $367-375$ & E 3 & E & 18 & 1.35 & 4 & $\begin{array}{l}\text { DN VALEN-[S } \\
\text { PF AVG] }\end{array}$ & $\begin{array}{c}\text { SECVRITAS } \\
\text { [REIPVBLICAE] }\end{array}$ & $\mathrm{R} \cdot \mathrm{TERTIA}$ & $\begin{array}{l}\text { RIC 121, } \\
24 \mathrm{~b}\end{array}$ & G60-217 & \\
\hline 484 & Valens & $367-375$ & Æ3 & $Æ$ & 17 & 2.21 & 5 & $\begin{array}{l}\text { DN VALEN-S } \\
\text { P[[F AVG }]\end{array}$ & $\begin{array}{c}\text { SECVRITAS [REI] } \\
\text { PVBLICAE }\end{array}$ & R TERTIA & $\begin{array}{c}\text { RIC } 121 \\
24 \mathrm{~b}\end{array}$ & G60-228 & \\
\hline 485 & Valens & $367-375$ & E 3 & EE & 18 & 2.38 & 1 & $\begin{array}{l}\text { DN VALEN-S } \\
\text { PF AVG }\end{array}$ & $\begin{array}{c}\text { SECVRITAS } \\
\text { REIPVB[LICAE] }\end{array}$ & $\begin{array}{c}\mathrm{R} \cdot \mathrm{SECVN}- \\
\mathrm{DA}\end{array}$ & $\begin{array}{c}\text { RIC } 121 \\
24 \mathrm{~b}\end{array}$ & G60-246 & \\
\hline 486 & Valens & $367-375$ & Æ3 & $Æ$ & 17 & 2.15 & 7 & $\begin{array}{l}\text { DN VALEN-S } \\
\text { PF AVG }\end{array}$ & $\begin{array}{c}\text { SECVR[ITAS] } \\
\text { REI[PVBLICAE] }\end{array}$ & $R \cdot$ QVARTA & $\begin{array}{l}\text { RIC 121, } \\
24 \mathrm{~b}\end{array}$ & G60-253 & \\
\hline 487 & Valens & $367-375$ & E 3 & $F$ & 18 & 2.44 & 5 & $\begin{array}{c}\text { [D]N VALEN-S } \\
\text { PF AVG }\end{array}$ & $\begin{array}{l}\text { [SE]CVR[ITAS] } \\
\text { REIPVBLICAE }\end{array}$ & R PRIMA & $\begin{array}{l}\text { RIC 121, } \\
24 \mathrm{~b}\end{array}$ & G60-263 & \\
\hline 488 & Valens & $367-375$ & Æ 3 & E & 18 & 2 & 7 & $\begin{array}{l}\text { DN VALEN-S } \\
\text { PF AVG }\end{array}$ & $\begin{array}{c}\text { SECVRITAS } \\
\text { REIPVBLICAE }\end{array}$ & $R \cdot P R I M A$ & $\begin{array}{c}\text { RIC 121, } \\
24 \mathrm{~b}\end{array}$ & G61-20 & $\begin{array}{l}\text { perfo- } \\
\text { rated }\end{array}$ \\
\hline 489 & Valens & $367-375$ & $\notin_{3}$ & $\mathscr{F}$ & 18 & 2.61 & 12 & $\begin{array}{l}\text { DN VALEN-S } \\
\text { PF AVG }\end{array}$ & $\begin{array}{l}\text { SECVRITAS } \\
\text { REIPVBLICAE }\end{array}$ & $\begin{array}{c}* \mathrm{~K} / \mathrm{Q} / / \mathrm{A} \\
\mathrm{SISC}\end{array}$ & $\begin{array}{c}\text { RIC 147, } \\
15 \mathrm{~b}\end{array}$ & C24035 & \\
\hline 490 & Valens & $367-375$ & $\notin 3$ & E & 17 & 2.17 & 1 & $\begin{array}{l}\text { DN VALEN-S } \\
\text { PF AVG }\end{array}$ & $\begin{array}{l}\text { S[EC]VRITAS } \\
\text { REIPVBLICAE }\end{array}$ & $\begin{array}{c}\text { RÁ/S } / / / A \\
\text { SIS C }\end{array}$ & $\begin{array}{c}\text { RIC 147, } \\
15 \mathrm{~b}\end{array}$ & C24073 & \\
\hline 491 & Valens & $367-375$ & $Æ_{3}$ & FE & 18 & 2.1 & 12 & $\begin{array}{l}\text { DN VALEN-S } \\
\text { PF AVG }\end{array}$ & $\begin{array}{l}\text { S[EC]VRITAS } \\
\text { REIPVBLICAE }\end{array}$ & $\begin{array}{c}\mathrm{DP} / \mathrm{F} / / \mathrm{A} \\
\mathrm{SISC}\end{array}$ & $\begin{array}{l}\text { RIC 147, } \\
15 \mathrm{~b}\end{array}$ & C24092 & \\
\hline 492 & Valens & $367-375$ & Æ 3 & E & 18 & 2.31 & 12 & $\begin{array}{l}\text { DN VALEN-S } \\
\text { PF AVG }\end{array}$ & $\begin{array}{l}\text { SECVRITAS } \\
\text { REIPVBLICAE }\end{array}$ & $\begin{array}{c}{ }^{*} \mathrm{~F} / \mathrm{M} / / \mathrm{A} \\
\mathrm{SISC}\end{array}$ & $\begin{array}{c}\text { RIC 147, } \\
15 \mathrm{~b}\end{array}$ & G60-2 & \\
\hline 493 & Valens & $367-375$ & $Æ_{3}$ & E & 19 & 1.68 & 1 & $\begin{array}{l}\text { [DN] VALEN- } \\
\text { [S PF AVG] }\end{array}$ & $\begin{array}{l}\text { S[ECVRITAS] } \\
\text { REIPVBLICAE }\end{array}$ & $\begin{array}{c}\mathrm{KA} / \mathrm{F} / /[\mathrm{A}] \\
\mathrm{SIS} C \mathrm{VE}\end{array}$ & $\begin{array}{l}\text { RIC 147, } \\
15 \mathrm{~b}\end{array}$ & G60-4 & \\
\hline 494 & Valens & $367-375$ & Æ 3 & E & 18 & 2.72 & 12 & $\begin{array}{l}\text { DN VALEN-S } \\
\text { PF AVG }\end{array}$ & $\begin{array}{l}\text { SECVRITAS } \\
\text { REIPVBLICAE }\end{array}$ & $\begin{array}{l}\text { CÁ/S } / / / A \\
\text { SIS C R }\end{array}$ & $\begin{array}{l}\text { RIC 147, } \\
15 \mathrm{~b}\end{array}$ & G6o-6 & \\
\hline 495 & Valens & $367-375$ & Æ 3 & $\mathscr{F}$ & 18 & 1.94 & 2 & $\begin{array}{l}\text { DN VALEN-S } \\
\text { PF AVG }\end{array}$ & $\begin{array}{l}\text { SECVRITAS } \\
\text { REIPVBLICAE }\end{array}$ & $\begin{array}{l}\mathrm{kK} / \mathrm{Q} / / \mathrm{A} \\
\mathrm{SISCE}\end{array}$ & $\begin{array}{l}\text { RIC 147, } \\
15 \mathrm{~b}\end{array}$ & G60-8 & \\
\hline 496 & Valens & $367-375$ & $\notin 3$ & $E$ & 18 & 1.96 & 6 & $\begin{array}{l}\text { DN VALEN-S } \\
\text { PF AVG }\end{array}$ & $\begin{array}{c}\text { SECVRITAS } \\
\text { REIPVBLICAE }\end{array}$ & $\begin{array}{c}* \mathrm{D} / \mathrm{S} / /[\mathrm{A}] \\
\mathrm{SISC}\end{array}$ & $\begin{array}{l}\text { RIC 147, } \\
15 \mathrm{~b}\end{array}$ & G60-16 & \\
\hline 497 & Valens & $367-375$ & $Æ_{3}$ & $F$ & 18 & 2.37 & 7 & $\begin{array}{l}\text { DN VALEN-S } \\
\text { PF AVG }\end{array}$ & $\begin{array}{l}\text { SECVRITAS } \\
\text { REIPVBLICAE }\end{array}$ & $\begin{array}{c}\mathrm{Rk} / \mathrm{F} / / \mathrm{A} \mathrm{SIS} \\
\mathrm{CVE}\end{array}$ & $\begin{array}{c}\text { RIC 147, } \\
15 \mathrm{~b}\end{array}$ & G60-23 & \\
\hline 498 & Valens & $367-375$ & $\notin 3$ & E & 18 & 2.72 & 6 & $\begin{array}{l}\text { DN VALEN-S } \\
\text { PF AVG }\end{array}$ & $\begin{array}{l}\text { [SECVRITAS] } \\
\text { REIPVBLICAE }\end{array}$ & $\begin{array}{l}\mathrm{R} /--/ / \cdot \mathrm{A} \\
\mathrm{SISC}\end{array}$ & $\begin{array}{l}\text { RIC 147, } \\
15 \mathrm{~b}\end{array}$ & G60-34 & \\
\hline 499 & Valens & $367-375$ & $\notin_{3} 3$ & E & 18 & 2.09 & 12 & $\begin{array}{c}\text { [DN VALEN]-S } \\
\text { PF AVG }\end{array}$ & $\begin{array}{l}\text { SECVRITAS } \\
\text { REIPVBLICAE }\end{array}$ & $\begin{array}{l}\mathrm{RÁ} / \mathrm{S} \cdot / / \mathrm{A} \\
\mathrm{SISC} \text { C }\end{array}$ & $\begin{array}{c}\text { RIC 147, } \\
15 \mathrm{~b}\end{array}$ & G60-49 & \\
\hline 500 & Valens & $367-375$ & E 3 & $F$ & 18 & 2.1 & 7 & $\begin{array}{l}\text { DN VALEN-S } \\
\text { PF AVG }\end{array}$ & $\begin{array}{l}\text { SECVRITAS } \\
\text { REIPVBLICAE }\end{array}$ & $\begin{array}{l}\mathrm{RK} / \mathrm{S} \cdot / / \mathrm{A} \\
\mathrm{SIS} \text { C E }\end{array}$ & $\begin{array}{c}\text { RIC 147, } \\
15 \mathrm{~b}\end{array}$ & G60-64 & \\
\hline 501 & Valens & $367-375$ & E 3 & $F$ & 18 & 2.03 & 12 & $\begin{array}{l}\text { DN VALEN-S } \\
\text { PF AVG }\end{array}$ & $\begin{array}{l}\text { SECVRITAS } \\
\text { REIPVBLICAE }\end{array}$ & $\begin{array}{c}* \mathrm{~K} / \mathrm{Q} / /[\mathrm{A}] \\
\mathrm{SIS} C \mathrm{R}\end{array}$ & $\begin{array}{c}\text { RIC 147, } \\
15 \mathrm{~b}\end{array}$ & G6o-67 & $\begin{array}{l}\text { perfo- } \\
\text { rated }\end{array}$ \\
\hline 502 & Valens & $367-375$ & $Æ_{3} 3$ & E & 18 & 1.92 & 1 & $\begin{array}{l}\text { DN VALEN-S } \\
\text { PF AVG }\end{array}$ & $\begin{array}{l}\text { SECVRITAS } \\
\text { REIPVBLICAE }\end{array}$ & $\begin{array}{c}* \mathrm{P} / \mathrm{M} / / \mathrm{A} \\
\mathrm{SISC}\end{array}$ & $\begin{array}{l}\text { RIC 147, } \\
\text { 15b }\end{array}$ & G60-82 & \\
\hline 503 & Valens & $367-375$ & $\notin 3$ & FE & 17 & 2.37 & 6 & $\begin{array}{l}\text { DN VALEN-S } \\
\text { PF AVG }\end{array}$ & $\begin{array}{c}\text { SECVRITAS } \\
\text { [REIPVBLICAE] }\end{array}$ & $\begin{array}{c}\mathrm{R} /--/ / \cdot \mathrm{A} \\
\mathrm{SISC}\end{array}$ & $\begin{array}{l}\text { RIC 147, } \\
15 \mathrm{~b}\end{array}$ & G60-85 & \\
\hline 504 & Valens & $367-375$ & $\notin 3$ & FE & 18 & 2.36 & 1 & $\begin{array}{l}\text { DN VALEN-S } \\
\text { PF AVG }\end{array}$ & $\begin{array}{l}\text { SECVRITAS } \\
\text { REIPVBLICAE }\end{array}$ & $\begin{array}{c}* \mathrm{RO} / \cdot \mathrm{M} / / \mathrm{A} \\
\mathrm{SISC}\end{array}$ & $\begin{array}{c}\text { RIC 147, } \\
15 \mathrm{~b}\end{array}$ & G60-87 & \\
\hline 505 & Valens & $367-375$ & Æ 3 & FE & 18 & 1.43 & 6 & $\begin{array}{l}\text { DN VALEN-S } \\
\text { PF AVG }\end{array}$ & $\begin{array}{c}\text { SECVRITAS } \\
\text { [REIPVB]LICAE }\end{array}$ & $\begin{array}{c}* \mathrm{~F} / \mathrm{M} / / \mathrm{A} \\
\mathrm{SISC}\end{array}$ & $\begin{array}{c}\text { RIC 147, } \\
15 \mathrm{~b}\end{array}$ & G60-128 & \\
\hline 506 & Valens & $367-375$ & Æ 3 & FE & 18 & 1.87 & 2 & $\begin{array}{c}\text { [DN VALEN]-S } \\
\text { PF AVG }\end{array}$ & $\begin{array}{l}\text { SECVRITAS } \\
\text { REIPVBLICAE }\end{array}$ & $\begin{array}{c}\mathrm{Rk} / \mathrm{F} / / \mathrm{A} S \mathrm{SIS} \\
\mathrm{CP}\end{array}$ & $\begin{array}{c}\text { RIC 147, } \\
15 \mathrm{~b}\end{array}$ & G60-129 & \\
\hline
\end{tabular}




\begin{tabular}{|c|c|c|c|c|c|c|c|c|c|c|c|c|c|}
\hline \multicolumn{14}{|c|}{ SOTIN } \\
\hline $\begin{array}{l}\text { CAT. } \\
\text { NO. }\end{array}$ & RULER & $\begin{array}{l}\text { DATING } \\
\text { (YRS) }\end{array}$ & DENOM. & MAT. & $\begin{array}{c}\text { D. } \\
(\mathrm{mm})\end{array}$ & $\begin{array}{l}\text { WT. } \\
\text { (g) }\end{array}$ & $\begin{array}{l}\text { ORI- } \\
\text { ENT. }\end{array}$ & OBVERSE & REVERSE & $\begin{array}{l}\text { MINT } \\
\text { MARKS }\end{array}$ & $\begin{array}{l}\text { REFE- } \\
\text { RENCE }\end{array}$ & $\begin{array}{l}\text { INV. } \\
\text { NO. }\end{array}$ & NOTE \\
\hline 507 & Valens & $367-375$ & E 3 & E & 18 & 2.07 & 1 & $\begin{array}{l}\text { DN VALEN-S } \\
\text { PF AV[G] }\end{array}$ & $\begin{array}{l}\text { SECVRITAS } \\
\text { REIPVBLICAE }\end{array}$ & $\begin{array}{l}{ }^{*} \mathrm{~F} / \mathrm{M} / / \mathrm{A} \\
\mathrm{SISC}\end{array}$ & $\begin{array}{l}\text { RIC 147, } \\
15 \mathrm{~b}\end{array}$ & G60-130 & \\
\hline 508 & Valens & $367-375$ & Æ3 & $\notin$ & 17 & 2.3 & 6 & $\begin{array}{c}\text { [DN VALEN]-S } \\
\text { PF AVG }\end{array}$ & $\begin{array}{l}\text { SECVRITAS } \\
\text { REIPVBLICAE }\end{array}$ & $\begin{array}{l}\mathrm{KP} / \mathrm{Q} / / \mathrm{A} \\
\mathrm{SISCR}\end{array}$ & $\begin{array}{l}\text { RIC 147, } \\
15 \mathrm{~b}\end{array}$ & G60-171 & \\
\hline 509 & Valens & $367-375$ & Æ 3 & E & 18 & 2.4 & 1 & $\begin{array}{c}\text { DN [VALEN]-S } \\
\text { PF AVG }\end{array}$ & $\begin{array}{c}\text { SECVRITAS } \\
\text { [REIPVBLICAE] }\end{array}$ & $\begin{array}{c}{ }^{*} \mathrm{~F} / \mathrm{M} / / \mathrm{A} \\
\text { SIS C }\end{array}$ & $\begin{array}{l}\text { RIC 147, } \\
15 \mathrm{~b}\end{array}$ & G60-281 & \\
\hline 510 & Valens & $367-375$ & $Æ 3$ & $\notin$ & 18 & 2.23 & 5 & $\begin{array}{l}\text { DN VALEN-S } \\
\text { PF AVG }\end{array}$ & $\begin{array}{l}\text { SECVRITAS } \\
\text { REIPVBLICAE }\end{array}$ & $\begin{array}{l}\mathrm{R} /--/ / \cdot \mathrm{A} \\
\text { SIS C }\end{array}$ & $\begin{array}{l}\text { RIC 147, } \\
15 \mathrm{~b}\end{array}$ & G61-1 & \\
\hline 511 & Valens & $367-375$ & $\notin_{3}$ & E & 18 & 2.64 & 12 & $\begin{array}{c}\text { DN VALEN-S } \\
\text { PF AVG }\end{array}$ & $\begin{array}{l}\text { SECVRITAS } \\
\text { REIPVBLICAE }\end{array}$ & $\begin{array}{l}* \mathrm{~F} / \mathrm{S} / / \mathrm{A} \\
\mathrm{SISC}\end{array}$ & $\begin{array}{l}\text { RIC 147, } \\
15 \mathrm{~b}\end{array}$ & G61-17 & \\
\hline 512 & Valens & $367-375$ & Æ3 & $Æ$ & 17 & 1.87 & 1 & $\begin{array}{c}\text { [DN VALEN]-S } \\
\text { PF AV[G] }\end{array}$ & $\begin{array}{c}\text { [SECVRI]TAS } \\
\text { RE[IPVBLICAE] }\end{array}$ & $\begin{array}{l}\text { D/--/?[A } \\
\text { SIS C] }\end{array}$ & $\begin{array}{l}\text { RIC } 147 \\
14 \mathrm{a}\end{array}$ & G61-53 & \\
\hline 513 & Valens & $367-375$ & Æ 3 & E & 17 & 2.04 & 1 & $\begin{array}{l}\text { DN VALEN-S } \\
\text { PF AVG }\end{array}$ & $\begin{array}{l}\text { SECVRITAS } \\
\text { REIPVBLICAE }\end{array}$ & $\begin{array}{c}* \mathrm{P} / \mathrm{M} / / \mathrm{A} \\
\text { SIS C }\end{array}$ & $\begin{array}{l}\text { RIC 147, } \\
15 \mathrm{~b}\end{array}$ & G67-9 & \\
\hline 514 & Valens & $367-375$ & Æ3 & $Æ$ & 18 & 2.22 & 7 & $\begin{array}{l}\text { DN VALEN-S } \\
\text { PF AVG }\end{array}$ & $\begin{array}{c}\text { [SECVRITA] } \\
\text { REIPV[BLICAE] }\end{array}$ & $\begin{array}{c}* \mathrm{P} / \mathrm{M} / / \mathrm{A} \\
\text { SIS C }\end{array}$ & $\begin{array}{l}\text { RIC 147, } \\
15 \mathrm{~b}\end{array}$ & G72-1 & \\
\hline 515 & Valens & $367-375$ & Æ 3 & E & 19 & 2.52 & 11 & $\begin{array}{l}\text { DN VALEN-S } \\
\text { PF AVG }\end{array}$ & $\begin{array}{c}\text { SECVRITAS } \\
\text { REIPVB[LICAE] }\end{array}$ & $* \mathrm{~V} / \Delta / / \mathrm{TES}$ & $\begin{array}{l}\text { RIC } 178 \\
27 \mathrm{~b}\end{array}$ & C24148 & \\
\hline 516 & Valens & $367-375$ & Æ3 & $Æ$ & 17 & 2.59 & 5 & $\begin{array}{c}\text { DN VALEN-[S } \\
\text { PF AVG] }\end{array}$ & $\begin{array}{c}\text { SECVRITAS } \\
\text { [REIPVBLICAE] }\end{array}$ & $* \Delta / ? / / \mathrm{TES}$ & $\begin{array}{l}\text { RIC } 178 \\
27 \mathrm{~b}\end{array}$ & G60-209 & \\
\hline 517 & Valens & $367-375$ & Æ 3 & EE & 17 & 2.01 & 5 & $\begin{array}{l}\text { DN VALENS } \\
\text { [PF AVG] }\end{array}$ & $\begin{array}{l}\text { SECVRITA[S REI] } \\
\text { PVBLIC[AE] }\end{array}$ & $\begin{array}{l}\cdot /--/ / \\
\text { cons S }\end{array}$ & $\begin{array}{l}\text { RIC 221, } \\
42 \mathrm{~b}\end{array}$ & G60-242 & \\
\hline 518 & Gratian & $367-375$ & $Æ 3$ & $\notin E$ & 18 & 2.51 & 12 & $\begin{array}{l}\text { DN GRATIA- } \\
\text { NVS PF AVG }\end{array}$ & $\begin{array}{l}\text { GLORIA RO-MA- } \\
\text { NORVM }\end{array}$ & $\begin{array}{l}\mathrm{M} / * \mathrm{~F} / / \Gamma \\
\mathrm{SISC}\end{array}$ & $\begin{array}{l}\text { RIC } 147, \\
14 \mathrm{C}\end{array}$ & C24251 & \\
\hline 519 & Gratian & $367-375$ & Æ 3 & $\mathscr{E}$ & 18 & 2.6 & 12 & $\begin{array}{l}\text { DN GRATIA- } \\
\text { NVS PF AVG }\end{array}$ & $\begin{array}{c}\text { GLORIA RO-MA- } \\
\text { NORVM }\end{array}$ & $\begin{array}{c}\mathrm{M} / * \mathrm{P} / / \Delta \\
\mathrm{SISC}\end{array}$ & $\begin{array}{c}\text { RIC 147, } \\
14 \mathrm{C}\end{array}$ & $\mathrm{C} 24280$ & \\
\hline 520 & Gratian & $367-375$ & $Æ 3$ & E & 19 & 2.45 & 2 & $\begin{array}{l}\text { DN GRATIA- } \\
\text { NVS PF AVG }\end{array}$ & $\begin{array}{l}\text { GLORIA RO- } \\
\text { [MANOR]VM }\end{array}$ & $\begin{array}{c}\mathrm{F} / \mathrm{DK} / / \Delta \mathrm{SIS} \\
\mathrm{CE}\end{array}$ & $\begin{array}{l}\text { RIC } 147, \\
14 \mathrm{C}\end{array}$ & C24415 & \\
\hline 521 & Gratian & $367-375$ & E 3 & $\mathscr{E}$ & 18 & 2.07 & 6 & $\begin{array}{l}\text { DN GRATIA- } \\
\text { NVS PF AVG }\end{array}$ & $\begin{array}{c}\text { GLORIA RO-MA- } \\
\text { NORVM }\end{array}$ & $\begin{array}{l}--/ R / / \cdot B \\
\text { SIS C }\end{array}$ & $\begin{array}{c}\text { RIC 147, } \\
14 \mathrm{C}\end{array}$ & G60-5 & \\
\hline 522 & Gratian & $367-375$ & $\notin 3$ & $Æ E$ & 19 & 2.41 & 6 & $\begin{array}{l}\text { DN GRATIA- } \\
\text { NVS PF AVG }\end{array}$ & $\begin{array}{l}\text { GLORIA RO-MA- } \\
\text { NORVM }\end{array}$ & $\begin{array}{l}\mathrm{M} / * \mathrm{~F} / / \Gamma \\
\mathrm{SISC}\end{array}$ & $\begin{array}{c}\text { RIC 147, } \\
14 \mathrm{C}\end{array}$ & G60-11 & \\
\hline 523 & Gratian & $367-375$ & Æ 3 & E & 18 & 2.03 & 12 & $\begin{array}{l}\text { DN GRATIA- } \\
\text { NVS PF AVG }\end{array}$ & $\begin{array}{c}\text { GLORIA RO-MA- } \\
\text { NORVM }\end{array}$ & $\begin{array}{l}--/ R / / \cdot B \\
\text { SIS C }\end{array}$ & $\begin{array}{c}\text { RIC 147, } \\
14 \mathrm{C}\end{array}$ & G60-17 & \\
\hline 524 & Gratian & $367-375$ & E 3 & AE & 19 & 2.41 & 7 & $\begin{array}{l}\text { DN GRATIA- } \\
\text { NVS PF AVG }\end{array}$ & $\begin{array}{l}\text { GLORIA RO-MA- } \\
\text { NORVM }\end{array}$ & $\begin{array}{l}\text { S./RÁ//D } \\
\text { SIS C V }\end{array}$ & $\begin{array}{l}\text { RIC } 147, \\
14 \mathrm{C}\end{array}$ & G60-36 & \\
\hline 525 & Gratian & $367-375$ & $Æ_{3}$ & E & 18 & 2.17 & 2 & $\begin{array}{l}\text { DN GRATIA- } \\
\text { NVS PF AVG }\end{array}$ & $\begin{array}{c}\text { GLORIA RO-MA- } \\
\text { NORVM }\end{array}$ & $\begin{array}{l}Q / K P / / \Delta \\
\text { SIS C R }\end{array}$ & $\begin{array}{c}\text { RIC 147, } \\
14 \mathrm{C}\end{array}$ & G60-53 & \\
\hline 526 & Gratian & $367-375$ & $\notin_{3} 3$ & $Æ E$ & 18 & 1.93 & 7 & $\begin{array}{l}\text { DN GRATIA- } \\
\text { NVS PF AVG }\end{array}$ & $\begin{array}{l}\text { GLORIA RO-MA- } \\
\text { NORVM }\end{array}$ & $\begin{array}{l}--/ R / / \cdot B \\
\text { SIS C }\end{array}$ & $\begin{array}{c}\text { RIC } 147, \\
14 \mathrm{C}\end{array}$ & G60-57 & \\
\hline 527 & Gratian & $367-375$ & $E_{3}$ & E & 17 & 1.78 & 12 & $\begin{array}{l}\text { DN GRATIA- } \\
\text { NVS PF AVG }\end{array}$ & $\begin{array}{l}\text { [GLOR]IA RO- } \\
\text { MANORVM }\end{array}$ & $\begin{array}{c}\mathrm{M} / * \mathrm{P} / / \Delta \\
\mathrm{SISC}\end{array}$ & $\begin{array}{l}\text { RIC 147, } \\
14 \mathrm{C}\end{array}$ & G60-61 & \\
\hline 528 & Gratian & $367-375$ & $\notin_{3} 3$ & $Æ E$ & 17 & 1.61 & 6 & $\begin{array}{l}\text { DN GRATIA- } \\
\text { NVS PF AVG }\end{array}$ & $\begin{array}{l}\text { GLORIA RO- } \\
\text { [MANO]RVM }\end{array}$ & $\begin{array}{l}\mathrm{Q} / * \mathrm{~K} / / \Delta \\
\mathrm{SISCR}\end{array}$ & $\begin{array}{l}\text { RIC } 147, \\
14 \mathrm{C}\end{array}$ & G60-91 & \\
\hline 529 & Gratian & $367-375$ & $\mathscr{E}_{3}$ & $E$ & 18 & 2.14 & 7 & $\begin{array}{l}\text { DN GRATIA- } \\
\text { NVS PF AVG }\end{array}$ & $\begin{array}{l}\text { GLORIA [RO]- } \\
\text { MANORVM }\end{array}$ & $\begin{array}{l}\mathrm{M} / * \mathrm{~F} / / \Gamma \\
\mathrm{SIS} \mathrm{C}\end{array}$ & $\begin{array}{c}\text { RIC 147, } \\
14 \mathrm{C}\end{array}$ & G60-97 & \\
\hline 530 & Gratian & $367-375$ & $\notin_{3} 3$ & $E$ & 18 & 1.74 & 1 & $\begin{array}{c}\text { [DN] GRA- } \\
\text { TIANVS PF } \\
\text { AV[G] }\end{array}$ & $\begin{array}{l}\text { GLORIA RO-MA- } \\
\text { NORVM }\end{array}$ & $\begin{array}{l}S \cdot / R A ́ / /[\Delta] \\
\text { SIS CE }\end{array}$ & $\begin{array}{l}\text { RIC 147, } \\
14 \mathrm{C}\end{array}$ & G60-104 & \\
\hline 531 & Gratian & $367-375$ & $E_{3} 3$ & $E$ & 19 & 2.36 & 7 & \begin{tabular}{|c} 
DN \\
GRATIANV[S \\
PF AVG]
\end{tabular} & $\begin{array}{l}\text { [GLOR]IA RO- } \\
\text { MA[NORVM] }\end{array}$ & $\begin{array}{l}\mathrm{M} / * \mathrm{~F} / / \Gamma \\
\mathrm{SISC}\end{array}$ & $\begin{array}{l}\text { RIC 147, } \\
14 \mathrm{C}\end{array}$ & G60-193 & \\
\hline 532 & Gratian & $367-375$ & E 3 & $E$ & 17 & 2.46 & 6 & $\begin{array}{l}\text { DN GRATIA- } \\
\text { NVS PF AVG }\end{array}$ & $\begin{array}{l}\text { GLORIA RO-MA- } \\
\text { NORVM }\end{array}$ & $\begin{array}{l}S \cdot / C A ́ / / \Delta \\
\text { SIS CA }\end{array}$ & $\begin{array}{l}\text { RIC } 147, \\
14 \mathrm{C}\end{array}$ & G60-211 & \\
\hline 533 & Gratian & $367-375$ & $E_{3}$ & $E$ & 19 & 2.74 & 7 & $\begin{array}{l}\text { [D]N GRATIA- } \\
\text { NVS PF AVG }\end{array}$ & $\begin{array}{l}\text { GLORIA RO- } \\
\text { [MANORVM] }\end{array}$ & $\begin{array}{l}\mathrm{M} / * \mathrm{~F} / / \Gamma \\
\mathrm{SISC}\end{array}$ & $\begin{array}{c}\text { RIC 147, } \\
14 \mathrm{C}\end{array}$ & G60-266 & \\
\hline 534 & Gratian & $367-375$ & $A_{3} 3$ & $E$ & 18 & 1.8 & 8 & $\begin{array}{l}\text { DN GRATIA- } \\
\text { NVS PF AVG }\end{array}$ & $\begin{array}{l}\text { GLORIA RO-MA- } \\
\text { NORVM }\end{array}$ & $\begin{array}{c}\cdot \mathrm{M} / * \mathrm{P} / / \Delta \\
\mathrm{SISC}\end{array}$ & $\begin{array}{l}\text { RIC } 147, \\
14 \mathrm{C}\end{array}$ & G60-272 & \\
\hline 535 & Gratian & $367-375$ & $\mathbb{E}_{3}$ & E & 18 & 2.04 & 2 & $\begin{array}{l}\text { DN GRATIA- } \\
\text { NVS PF AVG }\end{array}$ & $\begin{array}{c}\text { GLORIA RO-MA- } \\
\text { NORVM }\end{array}$ & $\begin{array}{l}\mathrm{M} / * \mathrm{P} / / \Gamma \\
\mathrm{SISC}\end{array}$ & $\begin{array}{c}\text { RIC 147, } \\
15 \mathrm{C}\end{array}$ & G61-4 & \\
\hline 536 & Gratian & $367-375$ & $E_{3}$ & EE & 17 & 1.95 & 7 & $\begin{array}{l}\text { DN GRATIA- } \\
\text { NVS PF AVG }\end{array}$ & $\begin{array}{l}\text { GLORIA RO-MA- } \\
\text { NORVM }\end{array}$ & $\begin{array}{c}--/ D / / * \Gamma \\
\text { SIS C }\end{array}$ & $\begin{array}{l}\text { RIC } 147, \\
14 \mathrm{C}\end{array}$ & G61-6 & \\
\hline
\end{tabular}




\begin{tabular}{|c|c|c|c|c|c|c|c|c|c|c|c|c|c|}
\hline \multicolumn{14}{|c|}{ SOTIN } \\
\hline $\begin{array}{l}\text { CAT. } \\
\text { NO. }\end{array}$ & RULER & $\begin{array}{l}\text { DATING } \\
\text { (YRS) }\end{array}$ & DENOM. & MAT. & $\begin{array}{c}\text { D. } \\
(\mathrm{mm})\end{array}$ & $\begin{array}{l}\text { WT. } \\
\text { (g) }\end{array}$ & $\begin{array}{l}\text { ORI- } \\
\text { ENT. }\end{array}$ & OBVERSE & REVERSE & $\begin{array}{l}\text { MINT } \\
\text { MARKS }\end{array}$ & $\begin{array}{l}\text { REFE- } \\
\text { RENCE }\end{array}$ & $\begin{array}{l}\text { INV. } \\
\text { NO. }\end{array}$ & NOTE \\
\hline 537 & Gratian & $367-375$ & E 3 & $\notin$ & 18 & 1.89 & 12 & $\begin{array}{c}\text { DN } \\
\text { GRA[TIAN]VS } \\
{[\text { PF AVG] }}\end{array}$ & $\begin{array}{l}\text { GLORIA [RO]- } \\
\text { MANOR[VM] }\end{array}$ & $\begin{array}{l}\mathrm{M} / * \mathrm{P} / / \Delta \\
\mathrm{SISC}\end{array}$ & $\begin{array}{l}\text { RIC } 147, \\
14 \mathrm{C}\end{array}$ & G61-33 & \\
\hline 538 & Gratian & $367-375$ & E 3 & E & 18 & 2.99 & 2 & $\begin{array}{l}\text { [DN] GRATIA- } \\
\text { NVS PF AVG }\end{array}$ & $\begin{array}{l}\text { GLORIA RO-MA- } \\
\text { NORVM }\end{array}$ & $\begin{array}{l}\mathrm{M} / \text { [F//厂 } \\
\mathrm{SISC}\end{array}$ & $\begin{array}{l}\text { RIC 147, } \\
14 \mathrm{C}\end{array}$ & G67-10 & \\
\hline 539 & Gratian & $367-375$ & Æ 3 & $\notin$ & 15 & 1.88 & 11 & $\begin{array}{c}\text { DN } \\
\text { GR[ATIANV]S } \\
\text { PF AVG }\end{array}$ & $\begin{array}{l}\text { GLORIA RO- } \\
\text { [MANORVM] }\end{array}$ & $\Gamma / \mathrm{P} / /[\mathrm{TES}]$ & $\begin{array}{l}\text { RIC } 178 \\
26 c\end{array}$ & G60-208 & \\
\hline 540 & Gratian & $367-375$ & E 3 & $E$ & 17 & 1.43 & 12 & $\begin{array}{c}\text { DN } \\
\text { GRATIAN[VS } \\
\text { PF AVG ] }\end{array}$ & $\begin{array}{l}\text { [GLORIA RO- } \\
\text { MANORVM] }\end{array}$ & $--/ \Gamma / /$ TES & $\begin{array}{l}\text { RIC 178, } \\
26 \mathrm{c}\end{array}$ & G60-139 & \\
\hline 541 & Gratian & $367-375$ & Æ 3 & $E$ & 18 & 2.27 & 5 & $\begin{array}{l}\text { DN GRATIA- } \\
\text { NVS PF AVG }\end{array}$ & $\begin{array}{c}\text { GLORIA RO-MA- } \\
\text { NORVM }\end{array}$ & $\mathrm{Z} / \cdot \mathrm{A} / / \mathrm{TES}$ & $\begin{array}{l}\text { RIC } 178 \\
26 \mathrm{c}\end{array}$ & G60-153 & \\
\hline 542 & Gratian & $367-375$ & $Æ 3$ & $\notin$ & 18 & 2.18 & 11 & $\begin{array}{l}\text { DN GRATIA- } \\
\text { NVS PF AVG }\end{array}$ & $\begin{array}{l}\text { GLORIA RO-MA- } \\
\text { NORVM }\end{array}$ & /回A//TES & $\begin{array}{l}\text { RIC 178, } \\
26 \mathrm{c}\end{array}$ & G60-111 & \\
\hline 543 & Gratian & $367-375$ & Æ 3 & 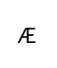 & 17 & 3.05 & 12 & $\begin{array}{l}\text { DN GRATIA- } \\
\text { NVS PF AVG }\end{array}$ & $\begin{array}{c}\text { GLORIA RO-MA- } \\
\text { NORVM }\end{array}$ & $--/ \mathrm{B} / / \mathrm{TES}$ & $\begin{array}{l}\text { RIC } 178 \\
26 \mathrm{C}\end{array}$ & C24436 & \\
\hline 544 & Gratian & $367-375$ & $E_{3}$ & $E$ & 19 & 2.29 & 12 & $\begin{array}{c}\text { [DN GR] } \\
\text { ATIANVS PF } \\
\text { AVG }\end{array}$ & $\begin{array}{l}\text { GLORIA RO- } \\
\text { MANO[RVM] }\end{array}$ & $\begin{array}{l}\text { ]/[B// } \\
{[T] E S}\end{array}$ & $\begin{array}{l}\text { RIC 178, } \\
26 c\end{array}$ & C24458 & \\
\hline 545 & Gratian & $367-375$ & $Æ 4$ & E & 16 & 1.83 & 4 & $\begin{array}{l}\text { [DN GRATIA] } \\
\text { NVS PF AVG }\end{array}$ & $\begin{array}{l}\text { [GLORIA] RO- } \\
\text { MANORVM }\end{array}$ & $\mathrm{V} / \mathrm{BB} / / \mathrm{TES}$ & $\begin{array}{l}\text { RIC } 178 \\
26 \mathrm{C}\end{array}$ & C24477 & \\
\hline 546 & Gratian & $367-375$ & $Æ 3$ & $\notin$ & 18 & 1.79 & 12 & $\begin{array}{c}\text { DN GRATA- } \\
\text { NVS PF [AVG] }\end{array}$ & $\begin{array}{l}\text { [GLORIA RO]- } \\
\text { MANORVM }\end{array}$ & V/㱏//TES & $\begin{array}{l}\text { RIC } 178 \\
26 \mathrm{C}\end{array}$ & C24478 & \\
\hline 547 & Gratian & $367-375$ & Æ 3 & $\notin$ & 18 & 2.31 & 12 & $\begin{array}{l}\text { DN GRATIA- } \\
\text { NVS PF AVG }\end{array}$ & $\begin{array}{l}\text { GLORIA RO-MA- } \\
\text { NORVM }\end{array}$ & $\begin{array}{l}\text { V/ ब्]Г// } \\
\text { [T]ES }\end{array}$ & $\begin{array}{l}\text { RIC } 178 \\
26 \mathrm{C}\end{array}$ & C24489 & \\
\hline 548 & Gratian & $367-375$ & Æ 3 & 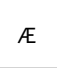 & 15 & 2.74 & 11 & $\begin{array}{l}\text { [DN GRATI]A- } \\
\text { NVS PF AVG }\end{array}$ & $\begin{array}{l}\text { GLORIA RO- } \\
\text { [MANORVM] }\end{array}$ & $?$ & incerta & G60-146 & \\
\hline 549 & Gratian & $367-375$ & Æ 3 & $\mathbb{E}$ & 15 & 1.08 & 6 & $\begin{array}{c}\text { [D]N } \\
\text { GRATI[ANVS } \\
\text { PF AVG] } \\
\end{array}$ & $\begin{array}{l}\text { SECVRITAS } \\
\text { REIPVBLICAE }\end{array}$ & R.PRIMA & $\begin{array}{l}\text { RIC } 121, \\
24 \mathrm{C}\end{array}$ & G61-60 & \\
\hline 550 & Gratian & $367-375$ & Æ 3 & $\notin$ & 19 & 1.87 & 6 & $\begin{array}{l}\text { DN GRATIA- } \\
\text { NVS PF AVG }\end{array}$ & $\begin{array}{l}\text { SECVRITAS } \\
\text { REIPVBLICAE }\end{array}$ & $\begin{array}{l}\mathrm{R} /--/ / \cdot \Delta \\
\text { SIS C }\end{array}$ & $\begin{array}{l}\text { RIC 147, } \\
15 \mathrm{C}\end{array}$ & C24614 & \\
\hline 551 & Gratian & $367-375$ & Æ3 & $\mathbb{E}$ & 17 & 2.4 & 12 & $\begin{array}{c}\text { D[N GRATI] } \\
\text { ANVS PF AVG }\end{array}$ & $\begin{array}{c}\text { SECVRITA[S } \\
\text { REIPVBLICAE] }\end{array}$ & $\begin{array}{l}\text { R/--//·A } \\
\text { SIS C }\end{array}$ & $\begin{array}{c}\text { RIC 147, } \\
15 \mathrm{C}\end{array}$ & G60-12 & \\
\hline 552 & Gratian & $367-375$ & Æ 3 & $\mathbb{E}$ & 16 & 1.94 & 1 & $\begin{array}{l}\text { DN GRATIA- } \\
\text { NVS PF AVG }\end{array}$ & $\begin{array}{l}\text { SECVRITAS } \\
\text { [REIPVBLICAE] }\end{array}$ & $\begin{array}{l}\text { D/--//回 A } \\
\text { SIS C }\end{array}$ & $\begin{array}{c}\text { RIC 147, } \\
15 \mathrm{C}\end{array}$ & G60-62 & \\
\hline 553 & Gratian & $367-375$ & $Æ_{3} 3$ & E & 18 & 3.02 & 7 & $\begin{array}{l}\text { DN GRATIA- } \\
\text { NVS PF AVG }\end{array}$ & $\begin{array}{l}\text { SECVRITAS } \\
\text { REIPVBLICAE }\end{array}$ & $\begin{array}{l}\text { D/--//国A } \\
\text { SIS C }\end{array}$ & $\begin{array}{c}\text { RIC 147, } \\
15 \mathrm{C}\end{array}$ & G60-284 & \\
\hline 554 & Gratian & $367-375$ & Æ 3 & $\notin$ & 18 & 2.25 & 6 & $\begin{array}{l}\text { DN GRATIA- } \\
\text { NVS PF AVG }\end{array}$ & $\begin{array}{l}\text { SECVRITAS } \\
\text { REIPVBLICAE }\end{array}$ & BB/当/TES & $\begin{array}{c}\text { RIC } 178 \\
27 \mathrm{C}\end{array}$ & G60-37 & \\
\hline 555 & Gratian & $367-375$ & Æ 3 & $\notin$ & 18 & 1.85 & 4 & $\begin{array}{l}\text { DN GRATIA- } \\
\text { NVS PF AVG }\end{array}$ & $\begin{array}{l}\text { SECVRITAS } \\
\text { REIPVBLICAE }\end{array}$ & Q $\mathrm{M} / \mathrm{B} / / \mathrm{TES}$ & $\begin{array}{l}\text { RIC } 178 \\
27 \mathrm{C}\end{array}$ & G60-38 & \\
\hline 556 & Gratian & $367-375$ & E 3 & 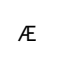 & 18 & 2.04 & 11 & $\begin{array}{l}\text { DN GRATIA- } \\
\text { NVS PF AVG }\end{array}$ & $\begin{array}{l}\text { SECVRITAS } \\
\text { REIPVBLICAE }\end{array}$ & $\begin{array}{l}\text { [A/l. wre- } \\
\text { ath//TES }\end{array}$ & $\begin{array}{c}\text { RIC } 178 \\
27 \mathrm{c}\end{array}$ & G60-219 & \\
\hline 557 & Valens & $375-378$ & Æ 3 & 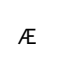 & 17 & 2.21 & 11 & $\begin{array}{l}\text { DN VALEN-S } \\
\text { PF AVG }\end{array}$ & $\begin{array}{l}\text { GLORIA RO- } \\
\text { MANO[RVM] }\end{array}$ & $\mathrm{V}$ /回B//TES & $\begin{array}{c}\text { RIC } 179 \\
31\end{array}$ & G60-1 & \\
\hline 558 & Gratian & $375-378$ & $\star_{3} 3$ & $\notin$ & 18 & 2.67 & 6 & $\begin{array}{l}\text { DN GRATIA- } \\
\text { NVS PF AVG }\end{array}$ & $\begin{array}{c}\text { GLORIA RO-MA- } \\
\text { NORVM }\end{array}$ & V/回 $/ / / T E S$ & $\begin{array}{c}\text { RIC } 179 \\
31\end{array}$ & C24488 & \\
\hline 559 & Gratian & $378-383$ & $Æ 4$ & $Æ$ & 16 & 1.94 & 11 & $\begin{array}{l}\text { [DN GRATIA]- } \\
\text { NVS PF AVG }\end{array}$ & $\begin{array}{l}\text { VICTOR-IA } \\
\text { AVGGG }\end{array}$ & SM R B & $\begin{array}{l}\text { RIC } 127 \\
48 \mathrm{a}\end{array}$ & G60-24 & \\
\hline 560 & Gratian & $378-383$ & $Æ 2$ & $\notin$ & 23 & 5.05 & 7 & $\begin{array}{l}\text { DN GRATIA- } \\
\text { NVS PF AVG }\end{array}$ & $\begin{array}{l}\text { [REPARAT]IO } \\
\text { REIPVB }\end{array}$ & B B SIS C & $\begin{array}{l}\text { RIC } 150 \\
26 \mathrm{a}\end{array}$ & G60-144 & \\
\hline 561 & Gratian & $378-383$ & $Æ 2$ & E & 24 & 4.75 & 12 & $\begin{array}{l}\text { DN GRATIA- } \\
\text { NVS PF AVG }\end{array}$ & $\begin{array}{l}\text { REPARATIO } \\
\text { REIPVB }\end{array}$ & Q A SIS C & $\begin{array}{l}\text { RIC } 150 \\
26 \mathrm{a}\end{array}$ & G61-9 & \\
\hline 562 & Gratian & $378-383$ & $Æ 4$ & $\notin$ & 19 & 1.48 & 6 & $\begin{array}{l}\text { [DN GRATIA]- } \\
\text { NVS PF AVG }\end{array}$ & $\begin{array}{c}\text { VOT } \mid \text { XV | MVLT } \\
\mid \mathrm{XX}\end{array}$ & ? & incerta & G60-282 & \\
\hline 563 & Gratian & $378-383$ & $\mathbb{E}_{4}$ & E & 15 & 1.18 & 7 & $\begin{array}{c}\text { [DN GR] } \\
\text { ATIA-[NV]S } \\
\text { PF AVG }\end{array}$ & $\begin{array}{c}\text { VOT | XV | MVLT } \\
\text { I }[\mathrm{XX}]\end{array}$ & ? & incerta & G61-43 & \\
\hline 564 & $\begin{array}{l}\text { Gratian - } \\
\text { Arcadius }\end{array}$ & $378-388$ & $Æ 4$ & $\notin$ & 12 & 1.11 & 5 & ? & $\begin{array}{c}\text { VOT | X | MVLT } \\
\mid \mathrm{XX}\end{array}$ & ? & incerta & G60-156 & \\
\hline 565 & $\begin{array}{l}\text { Gratian - } \\
\text { Arcadius }\end{array}$ & $378-383$ & Æ3 & $\notin$ & 19 & 3.02 & 9 & ? & $\begin{array}{c}\text { CONCOR-DIA } \\
\text { AVGGG }\end{array}$ & $?$ & incerta & G60-183 & \\
\hline
\end{tabular}




\begin{tabular}{|c|c|c|c|c|c|c|c|c|c|c|c|c|c|}
\hline \multicolumn{14}{|c|}{ SOTIN } \\
\hline $\begin{array}{l}\text { CAT. } \\
\text { NO. }\end{array}$ & RULER & $\begin{array}{l}\text { DATING } \\
\text { (YRS) }\end{array}$ & DENOM. & MAT. & $\begin{array}{c}\text { D. } \\
(\mathrm{mm})\end{array}$ & $\begin{array}{l}\text { WT. } \\
\text { (g) }\end{array}$ & $\begin{array}{l}\text { ORI- } \\
\text { ENT. }\end{array}$ & OBVERSE & REVERSE & $\begin{array}{c}\text { MINT } \\
\text { MARKS }\end{array}$ & $\begin{array}{l}\text { REFE- } \\
\text { RENCE }\end{array}$ & $\begin{array}{l}\text { INV. } \\
\text { NO. }\end{array}$ & NOTE \\
\hline 566 & Valentinian II & $378-383$ & Æ 2 & $Æ$ & 25 & 5.35 & 1 & $\begin{array}{l}\text { DN VALEN- } \\
\text { TINIANVS PF } \\
\text { AVG }\end{array}$ & $\begin{array}{c}\text { REPARATIO } \\
\text { R[EIPVB] }\end{array}$ & SM R $€$ & $\begin{array}{l}\text { RIC 126, } \\
43 \mathrm{C}\end{array}$ & G2342-2 & \\
\hline 567 & Valentinian II & $378-383$ & Æ 2 & ÆE & 24 & 5.12 & 1 & $\begin{array}{l}\text { DN VALENTI- } \\
\text { NIANVS IVN } \\
\text { PF AVG }\end{array}$ & $\begin{array}{l}\text { REPARATIO } \\
\text { REIPVB }\end{array}$ & B SIS C. & $\begin{array}{l}\text { RIC 150, } \\
26 \mathrm{~b}\end{array}$ & C24856 & $\begin{array}{l}\text { Alföldi } \\
1924\end{array}$ \\
\hline 568 & Valentinian II & $378-383$ & Æ 2 & $Æ$ & 22 & 5.52 & 7 & $\begin{array}{l}\text { DN VALENTI- } \\
\text { NIANVS IVN } \\
\text { PF AVG }\end{array}$ & $\begin{array}{l}\text { [REPARATIO] } \\
\text { REIPVB }\end{array}$ & ? SIS C. & $\begin{array}{l}\text { RIC 150, } \\
26 b\end{array}$ & G2342-1 & \\
\hline 569 & Valentinian II & $378-383$ & Æ 3 & $\notin E$ & 19 & 2.39 & 6 & $\begin{array}{l}\text { DN VALEN- } \\
\text { TINIANVS PF } \\
\text { AVG }\end{array}$ & $\begin{array}{c}\text { CONCOR-DIA } \\
\text { AVGGG }\end{array}$ & A SIS C & $\begin{array}{l}\text { RIC 151, } \\
27 \mathrm{~b}\end{array}$ & C24742 & $\begin{array}{c}\text { Alföldi } \\
1924\end{array}$ \\
\hline 570 & Valentinian II & $378-383$ & Æ 3 & $Æ$ & 19 & 2.03 & 6 & $\begin{array}{c}\text { DN VAL[EN] } \\
\text { TINIANVS PF } \\
\text { AVG }\end{array}$ & $\begin{array}{c}\text { CONCOR-DIA } \\
\text { AVGGG }\end{array}$ & B SIS C & $\begin{array}{l}\text { RIC 151, } \\
27 \mathrm{~b}\end{array}$ & G60-33 & \\
\hline 571 & Valentinian II & $378-383$ & Æ 4 & $Æ$ & 13 & 0.7 & 6 & $\begin{array}{l}\text { DN VALENTI- } \\
\text { NIANVS IVN } \\
\text { PF AVG }\end{array}$ & $\begin{array}{c}\text { VOT | V | MVLT } \\
\text { |X }\end{array}$ & * B SIS C & $\begin{array}{l}\text { RIC 152, } \\
29 \mathrm{C}\end{array}$ & G60-225 & \\
\hline 572 & Valentinian II & $378-383$ & E 4 & $Æ$ & 14 & 1.35 & 12 & $\begin{array}{l}\text { DN VALEN- } \\
\text { TINIANVS PF } \\
\text { AVG }\end{array}$ & $\begin{array}{c}\text { VOT | X | MVLT } \\
\text { | XX }\end{array}$ & $\operatorname{CON} \Delta$ & $\begin{array}{l}\text { RIC 229, } \\
63 a\end{array}$ & C24977 & \\
\hline 573 & Valentinian II & $378-383$ & Æ 4 & $Æ$ & 12 & 0.5 & 6 & $\begin{array}{c}\text { [DN VALENT] } \\
\text { INIANVS PF } \\
\text { AVG }\end{array}$ & $\begin{array}{c}\text { VOT } \mid \text { X | MVLT } \\
\text { IXX }\end{array}$ & $?$ & incerta & G60-154 & \\
\hline 574 & Valentinian II & $378-383$ & Æ 3 & $Æ$ & 18 & 2.02 & 6 & DN VALEN ? & $\begin{array}{c}\text { CONCOR-D }[I A A] \\
\text { VGGG }\end{array}$ & $?$ & incerta & G60-169 & \\
\hline 575 & Theodosius I & $379-383$ & Æ 2 & $Æ$ & 23 & 4.83 & 6 & $\begin{array}{l}\text { DN THEODO- } \\
\text { [SIVS PF] } \\
\text { AVG }\end{array}$ & $\begin{array}{l}\text { REPARATIO } \\
\text { R[EIPVB] }\end{array}$ & * B SIS [C] & $\begin{array}{l}\text { RIC 150, } \\
26 \mathrm{c}\end{array}$ & G60-80 & \\
\hline 576 & Theodosius I & $379-383$ & Æ 3 & $Æ$ & 19 & 2.06 & 1 & $\begin{array}{l}\text { DN THEODO- } \\
\text { SIVS PF AVG }\end{array}$ & $\begin{array}{c}\text { CONCOR-[DIA] } \\
\text { AVGGG }\end{array}$ & B SIS C & $\begin{array}{l}\text { RIC 151, } \\
27 d\end{array}$ & G60-100 & \\
\hline 577 & Theodosius I & $379-383$ & A 4 & $Æ$ & 15 & 1.08 & 7 & $\begin{array}{l}\text { DN THEODO- } \\
\text { SIVS PF AVG }\end{array}$ & $\begin{array}{c}\text { VOT | V | MVLT } \\
\mid \mathrm{X}\end{array}$ & A SIS C & $\begin{array}{l}\text { RIC 152, } \\
\text { 29d }\end{array}$ & G60-9 & \\
\hline 578 & Theodosius I & $379-383$ & Æ 4 & $Æ$ & 15 & 1.18 & 1 & $\begin{array}{l}\text { DN THEODO- } \\
\text { [SIVS] PF } \\
\text { AVG }\end{array}$ & $\begin{array}{c}\text { VOT | V | MVLT } \\
\text { |X }\end{array}$ & A SIS C. & $\begin{array}{l}\text { RIC 152, } \\
\text { 29d }\end{array}$ & G60-28 & \\
\hline 579 & Theodosius I & $379-383$ & Æ 4 & $Æ$ & 15 & 1.51 & 1 & $\begin{array}{l}\text { DN THEODO- } \\
\text { SIVS PF AVG }\end{array}$ & $\begin{array}{c}\text { VOT | V | MVLT } \\
\mid \mathrm{X}\end{array}$ & A SIS C & $\begin{array}{l}\text { RIC 152, } \\
29 d\end{array}$ & G60-88 & \\
\hline 580 & Theodosius I & $379-383$ & Æ 4 & $Æ$ & 15 & 1.59 & 1 & $\begin{array}{l}\text { DN THEODO- } \\
\text { SIVS PF AVG }\end{array}$ & $\begin{array}{l}\text { [VOT | V |] } \\
\text { MVLT |X }\end{array}$ & A SIS C. & $\begin{array}{l}\text { RIC 152, } \\
29 d\end{array}$ & G60-188 & \\
\hline 581 & Theodosius I & $379-383$ & Æ 4 & $Æ$ & 16 & 2.55 & 1 & $\begin{array}{l}\text { DN THEODO- } \\
\text { SIVS PF AVG }\end{array}$ & $\begin{array}{c}\text { VOT } \mid \text { X | MVLT } \\
\mid \mathrm{XX}\end{array}$ & A SIS C & $\begin{array}{l}\text { RIC 152, } \\
30 b\end{array}$ & G60-123 & \\
\hline 582 & Theodosius I & $379-383$ & Æ 4 & $Æ$ & 14 & 1.3 & 11 & $\begin{array}{l}\text { DN THEODO- } \\
\text { SIVS PF AVG }\end{array}$ & $\begin{array}{c}\text { VOT | X | MVLT } \\
\mid \mathrm{XX}\end{array}$ & SM HA & $\begin{array}{c}\text { RIC 196, } \\
19 \mathrm{C}\end{array}$ & C25272 & $\begin{array}{c}\text { Alföldi } \\
1924\end{array}$ \\
\hline 583 & Theodosius I & $379-383$ & Æ 4 & $Æ$ & 17 & 1.57 & 7 & $\begin{array}{l}\text { DN THEODO- } \\
\text { SIVS PF AVG }\end{array}$ & $\begin{array}{c}\text { VOT } \mid \text { X | MVLT } \\
\mid X X\end{array}$ & SM K Г & $\begin{array}{l}\text { RIC 244, } \\
21 \mathrm{C}\end{array}$ & G60-113 & \\
\hline 584 & Theodosius I & $379-388$ & Æ 4 & $Æ$ & 15 & 1.2 & 1 & $\begin{array}{c}\text { DN } \\
\text { THE[ODO]- } \\
\text { SIVS PF AVG }\end{array}$ & $\begin{array}{c}\text { VOT | V | MVLT } \\
\mid \mathrm{X}\end{array}$ & $?$ & incerta & G60-198 & \\
\hline 585 & Theodosius I & $379-388$ & Æ 4 & $Æ$ & 13 & 1.16 & 5 & $\begin{array}{l}\text { DN THEODO- } \\
\text { SIVS PF AVG }\end{array}$ & [VOT|?|M]VLT|? & $?$ & incerta & G60-278 & \\
\hline 586 & Gratian & $381-383$ & Æ 4 & $Æ$ & 14 & 1.83 & 7 & $\begin{array}{l}\text { DN GRATIA- } \\
\text { NVS PF AVG }\end{array}$ & $\begin{array}{c}\text { VOT | XV | MVLT } \\
\mid X X\end{array}$ & SIS C & LRBC 1537 & G60-7 & \\
\hline 587 & Gratian & $381-383$ & $Æ 4$ & 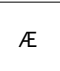 & 15 & 1.51 & 2 & $\begin{array}{l}\text { DN GRATIA- } \\
\text { NVS PF AVG }\end{array}$ & $\begin{array}{c}\text { VOT | XV | MVLT } \\
\text { |XX }\end{array}$ & B SIS C & LRBC 1539 & G60-13 & \\
\hline 588 & Gratian & $381-383$ & Æ 4 & $Æ$ & 15 & 1.13 & 12 & $\begin{array}{l}\text { DN GRATIA- } \\
\text { NVS PF AVG }\end{array}$ & $\begin{array}{c}\text { VOT }|X V| \text { MVLT } \\
\mid X X\end{array}$ & SIS C & LRBC 1537 & G60-18 & \\
\hline 589 & Gratian & $381-383$ & $Æ 4$ & $Æ$ & 15 & 1.07 & 7 & $\begin{array}{l}\text { DN GRATIA- } \\
\text { NVS PF AVG }\end{array}$ & $\begin{array}{c}\text { VOT | XV | MVLT } \\
\mid \mathrm{XX}\end{array}$ & B SIS C. & LRBC 1547 & G60-29 & \\
\hline 590 & Gratian & $381-383$ & Æ 4 & $Æ$ & 16 & 1.5 & 6 & $\begin{array}{l}\text { DN GRATIA- } \\
\text { NVS PF AVG }\end{array}$ & $\begin{array}{c}\text { VOT | XV | MVLT } \\
\mid X X\end{array}$ & B SIS C & LRBC 1539 & G60-105 & \\
\hline 591 & Gratian & $381-383$ & $Æ 4$ & $Æ$ & 15 & 1.6 & 7 & $\begin{array}{l}\text { DN GRATIA- } \\
\text { NVS PF AVG }\end{array}$ & $\begin{array}{c}\text { VOT | XV | MVLT } \\
\mid X X\end{array}$ & A SIS C. & LRBC 1547 & G60-112 & \\
\hline 592 & Gratian & $381-383$ & Æ 4 & $Æ$ & 14 & 1.66 & 1 & $\begin{array}{l}\text { DN GRATIA- } \\
\text { NVS PF AVG }\end{array}$ & $\begin{array}{c}\text { VOT | XV | MVLT } \\
\mid \mathrm{XX}\end{array}$ & B SIS C & LRBC 1539 & G60-124 & \\
\hline 593 & Gratian & $381-383$ & Æ 4 & $Æ$ & 17 & 1.48 & 6 & $\begin{array}{l}\text { DN GRATIA- } \\
\text { NVS PF AVG }\end{array}$ & $\begin{array}{c}\text { VOT | [X]V | } \\
\text { MVLT |XX }\end{array}$ & A SIS C & LRBC 1539 & G60-131 & \\
\hline
\end{tabular}




\begin{tabular}{|c|c|c|c|c|c|c|c|c|c|c|c|c|c|}
\hline \multicolumn{14}{|c|}{ SOTIN } \\
\hline $\begin{array}{l}\text { CAT. } \\
\text { NO. }\end{array}$ & RULER & $\begin{array}{l}\text { DATING } \\
\text { (YRS) }\end{array}$ & DENOM. & MAT. & $\begin{array}{c}\mathrm{D} . \\
(\mathrm{mm})\end{array}$ & $\begin{array}{l}\text { WT. } \\
\text { (g) }\end{array}$ & $\begin{array}{l}\text { ORI- } \\
\text { ENT. }\end{array}$ & OBVERSE & REVERSE & $\begin{array}{c}\text { MINT } \\
\text { MARKS }\end{array}$ & $\begin{array}{l}\text { REFE- } \\
\text { RENCE }\end{array}$ & $\begin{array}{l}\text { INV. } \\
\text { NO. }\end{array}$ & NOTE \\
\hline 594 & Gratian & $381-383$ & Æ 4 & Æ & 15 & 1.31 & 6 & $\begin{array}{l}\text { DN GRATIA- } \\
\text { NVS PF AVG }\end{array}$ & $\begin{array}{c}\text { VOT } \mid \text { XV | MVLT } \\
\mid \mathrm{XX}\end{array}$ & SIS C & LRBC 1537 & G61-10 & \\
\hline 595 & Gratian & $381-383$ & Æ 4 & $Æ$ & 15 & 1.26 & 1 & $\begin{array}{l}\text { DN GRATIA- } \\
\text { NVS PF AVG }\end{array}$ & $\begin{array}{c}\text { VOT | X[V] | } \\
\text { MVLT |XX }\end{array}$ & B SIS C & LRBC 1539 & G61-31 & \\
\hline 596 & Arcadius & 383 & Æ 4 & $Æ$ & 13 & 1.36 & 6 & $\begin{array}{l}\text { [DN ARCA- } \\
\text { DIVS PF AVG }\end{array}$ & VOT | X & CON $\Gamma$ & $\begin{array}{l}\text { RIC } 229 \\
62 b\end{array}$ & G60-43 & \\
\hline 597 & Aelia Flaccilla & $383-386$ & $Æ 4$ & ÆE & 14 & 0.96 & 6 & $\begin{array}{l}\text { AEL FLAC- } \\
\text { CILLA AVG }\end{array}$ & $\begin{array}{l}\text { SALVS REI- } \\
\text { PVB[LICAE] }\end{array}$ & $?$ & incerta & G60-70 & \\
\hline 598 & Theodosius I & $383-388$ & Æ 4 & $Æ$ & 14 & 1 & 5 & $\begin{array}{l}\text { DN THEODO- } \\
\text { SIVS PF A[VG] }\end{array}$ & $\begin{array}{c}\text { VICTOR[-IA } \\
\text { AVGGG] }\end{array}$ & $\mathrm{R} Q$ & $\begin{array}{c}\text { RIC 131, } \\
56 \mathrm{C}\end{array}$ & C25233 & \\
\hline 599 & Arcadius & $383-388$ & Æ 4 & $Æ$ & 13 & 0.73 & 5 & $\begin{array}{c}\text { DN ARCADIVS } \\
\text { [PF AVG] }\end{array}$ & VOT|V & $?$ & incerta & G60-226 & \\
\hline 600 & Arcadius & $383-388$ & $Æ 4$ & $\not E$ & 16 & 1.17 & 7 & $\begin{array}{l}\text { ? [ARCA] } \\
\text { DI-[VS]? }\end{array}$ & $?$ & $?$ & incerta & G60-264 & \\
\hline 601 & $\begin{array}{l}\text { Theod. I - } \\
\text { Arcadius }\end{array}$ & $383-403$ & Æ 4 & E & 13 & 1 & 6 & $?$ & $\begin{array}{l}\text { [SALV]S REI- } \\
\text { PVBLI[CAE] }\end{array}$ & ANT & incerta & G60-194 & \\
\hline 602 & $\begin{array}{l}\text { Theod. I - } \\
\text { Honorius }\end{array}$ & $383-403$ & Æ 4 & E & 14 & 1.29 & 5 & ? & $\begin{array}{l}\text { [SALVS] REI- } \\
\text { PVBLICAE }\end{array}$ & $\mathrm{p} /--/ /$ ? & incerta & G60-220 & \\
\hline 603 & $\begin{array}{l}\text { Theod. I - } \\
\text { Honorius }\end{array}$ & $383-403$ & Æ4 & Æ & 13 & 1.4 & 5 & $?$ & $\begin{array}{l}\text { [SALVS REI- } \\
\text { PVBLICAE] }\end{array}$ & $\mathrm{P} /--/ /$ ? & incerta & G61-49 & \\
\hline 604 & Valentinian II & $385-386$ & $Æ 4$ & A & 14 & 1.24 & 1 & $\begin{array}{c}\text { DN VALENTI- } \\
\text { NI-ANVS PF } \\
\text { AVG }\end{array}$ & $\begin{array}{l}\text { VICTOR-IA } \\
\text { AVGGG }\end{array}$ & A SIS & LRBC 1575 & C24927 & \\
\hline 605 & Valentinian II & $385-386$ & Æ 4 & AE & 14 & 1.13 & 12 & $\begin{array}{c}\text { DN VALENTI- } \\
\text { NI-ANVS PF } \\
\text { AVG }\end{array}$ & $\begin{array}{l}\text { VICTOR-IA } \\
\text { AVG[GG] }\end{array}$ & A SIS & LRBC 1575 & G60-58 & \\
\hline 606 & Valentinian II & $385-386$ & $\notin 4$ & Æ & 14 & 0.89 & 5 & $\begin{array}{c}\text { DN VALENTI- } \\
\text { NI-ANVS PF } \\
\text { AVG }\end{array}$ & $\begin{array}{c}\text { [VICTOR]-IA } \\
\text { AVGGG }\end{array}$ & A SIS & LRBC 1575 & G60-103 & \\
\hline 607 & Valentinian II & $385-386$ & $\notin 4$ & $Æ E$ & 14 & 0.89 & 5 & $\begin{array}{c}\text { DN VALENTI- } \\
\text { NI-ANVS [PF] } \\
\text { AVG }\end{array}$ & $\begin{array}{l}\text { [VICTOR]-IA } \\
\text { AVGGG }\end{array}$ & A SIS & LRBC 1575 & G60-135 & \\
\hline 608 & Theodosius I & $384 ?-387$ & E 4 & AE & 14 & 1.39 & 2 & $\begin{array}{l}\text { DN THEODO- } \\
\text { SIVS PF AVG }\end{array}$ & $\begin{array}{l}\text { VICTOR-IA } \\
\text { AVGGG }\end{array}$ & A SIS & $\begin{array}{l}\text { RIC 155, } \\
39 b\end{array}$ & G60-133 & \\
\hline 609 & Theodosius I & $384 ?-387$ & E 4 & Æ & 14 & 0.89 & 6 & $\begin{array}{l}\text { DN THEODO- } \\
\text { SIVS PF AVG }\end{array}$ & $\begin{array}{c}\text { VICTOR-IA } \\
\text { AVGGG }\end{array}$ & B SIS . & $\begin{array}{c}\text { RIC } 155 \\
39 \mathrm{~b}\end{array}$ & G60-134 & \\
\hline 610 & Arcadius & $384 ?-387$ & E 3 & Æ & 18 & 1.77 & 7 & $\begin{array}{l}\text { DN ARCADI- } \\
\text { VS PP AVG }\end{array}$ & $\begin{array}{c}\text { GLORIA RO-MA- } \\
\text { NORVM }\end{array}$ & B SIS C & $\begin{array}{c}\text { RIC 154 } \\
38 \mathrm{C}\end{array}$ & D70 & \\
\hline 611 & Arcadius & $384 ?-387$ & $\mathbb{E}_{4}$ & AE & 14 & 0.97 & 6 & $\begin{array}{c}\text { DN ARCADIVS } \\
\text { PF AVG }\end{array}$ & $\begin{array}{l}\text { VICTOR-IA } \\
\text { AVGGG }\end{array}$ & A SIS & $\begin{array}{l}\text { RIC 155, } \\
39 \mathrm{C}\end{array}$ & $\mathrm{D} 152$ & \\
\hline 612 & Theodosius I & $384-388$ & $Æ 3$ & A & 17 & 1.9 & 11 & $\begin{array}{l}\text { DN THEODO- } \\
\text { SIVS PF AVG }\end{array}$ & VIRTVS AVGGG & $\mathrm{A} /-/ / \mathrm{TES}$ & $\begin{array}{l}\text { RIC } 186, \\
61 b\end{array}$ & C25241 & \\
\hline 613 & Theodosius I & $384-388$ & Æ 3 & A & 20 & 1.5 & 6 & $\begin{array}{l}\text { DN THEODO- } \\
\text { SIVS PF AVG }\end{array}$ & VIRTVS AVGGG & A/--//TES & $\begin{array}{l}\text { RIC 186, } \\
61 \mathrm{~b}\end{array}$ & G60-147 & \\
\hline 614 & Arcadius & $384-388$ & $E_{4}$ & AE & 13 & 1.47 & 12 & $\begin{array}{c}\text { DN ARCADIVS } \\
\text { PF AVG }\end{array}$ & VICTORIA AVG & TES $\Gamma$ & $\begin{array}{l}\text { RIC 187, } \\
62 \mathrm{C}\end{array}$ & D105 & \\
\hline 615 & Arcadius & $384-388$ & $\mathbb{E}_{4}$ & Æ & 11 & 0.84 & 11 & $\begin{array}{c}\text { [DN } \\
\text { THEODO]- } \\
\text { SIVS PF AVG }\end{array}$ & $\begin{array}{l}\text { [GLORIA REI]- } \\
\text { PVBLICE }\end{array}$ & [TES] & incerta & G60-223 & \\
\hline 616 & Valentinian II & $388-392$ & $\mathbb{E}_{4}$ & AE & 14 & 1.08 & 6 & $\begin{array}{c}\text { DN VALENTI- } \\
\text { NI-ANVS PF } \\
\text { AVG }\end{array}$ & $\begin{array}{l}\text { SALVS REI- } \\
\text { PVBLICA[E] }\end{array}$ & $A Q S$ & $\begin{array}{l}\text { RIC 106, } \\
58 \mathrm{a}\end{array}$ & C24880 & \\
\hline 617 & Valentinian II & $388-392$ & $Æ 4$ & Æ & 14 & 1.29 & 12 & $\begin{array}{c}\text { DN VALENTI- } \\
\text { NI-[ANVS PF } \\
\text { AVG] }\end{array}$ & $\begin{array}{l}\text { SALVS REI-PVBLI- } \\
\text { CAE }\end{array}$ & $P /--/ / R \cdot P$ & $\begin{array}{l}\text { RIC 133, } \\
64 \mathrm{a}\end{array}$ & G60-116 & \\
\hline 618 & Arcadius & $388-392$ & $\notin 4$ & A & 12 & 0.82 & 12 & $\begin{array}{l}\text { DN [ARCADI] } \\
\text { VS PF AVG }\end{array}$ & $\begin{array}{l}\text { SALVS REI- } \\
\text { [PVBLICAE] }\end{array}$ & $\begin{array}{c}\mathrm{P} /--/ / \mathrm{SM} \\
\mathrm{K \Gamma}\end{array}$ & $\begin{array}{c}\text { RIC } 246, \\
26 c\end{array}$ & G60-71 & \\
\hline
\end{tabular}




\section{BIBLIOGRAPHY}

AE - M. Besnier, R. Cagnat, A. Chastagnol, M. Corbier, S. Dardaine, J. Gagé, M Le Glay. A. Merlin. A. Laronde, P. Le Roux, H. G. Pflaum, P. Wuilleumier, L'Année épigraphique, Centre national de la recherche scientifique, 1888-2013.

Alföldi 1924 - A. Alföldi, Der Untergang der Römerherrschaft in Pannonien 1 Ungarische Bibliothek für das Ungarische Institut an der Universität Berlin 10, Walter de Gruyter \& Co., 1924.

Alföldi 1924 - A. Alföldi, Der Untergang der Römerherrschaft in Pannonien 2 Ungarische Bibliothek für das Ungarische Institut an der Universität Berlin 12 Walter de Gruyter \& Co., 1926.

Banaji 2002 - J. Banaji, Agrarian Change in Late Antiquity Gold, Labour, and Aristocratic Dominance, Oxford Classical Monographs, Oxford University Press, 2002.

Bilić 2018 - T. Bilić, Predcarski novac iz Siska, in Drnić, I. (ed.), Segestika i Siscija - od ruba imperija do provincijskog središta / Segestica and Siscia - from the Periphery of the Empire to a Provincial Center, Arheološki muzej u Zagrebu, 2018, 267-277

Bratož 2011- R. Bratož, Die Auswanderung der Bevölkerung aus den pannonischen Provinzen während des 5. und 6. Jahrhunderts, in Konrad, M., Witschel, Ch. (eds.), Römische Legionslager in den Rhein- und Donauprovinzen-Nuclei spätantik-frühmittelalterlichen Lebens?, Abhandlungen 138, Bayerische Akademie der Wissenschaften, Philosophisch-historische Klasse, Neue Folge, 2011, 589-614

Brunšmid 1911 - J. Brunšmid, Nahođaj rimskih denara I. i II. stoljeća u Sotinu, Vijesnik za historiju i arheologiju Dalmatinsku 11, 1911, 241-277.

Bulat 1969 - M. Bulat, Topografska istraživanja limesa u Slavoniji i Baranji, Osječki zbornik 12, 1969, 39-52.

Bulat 1974 - M. Bulat, Dalj - antičko naselje, Arheološki pregled 16, 1974, 85-86.

Bulat 1977 - M. Bulat, Ciglana u Dalju - rimski grob od opeka, Arheološki pregled 19, 1977, 93-95.

Burger 1981 - A. S. Burger, Late Roman Money Circulation in South-Pannonia, Régészeti Füzetek 2/22, Magyar Nemzeti Múzeum, 1981.

Burns 1994 - T. S. Burns, Barbarians within the Gates of Rome. A Study of Roman Military Policy and the Barbarians, ca. 375-425 A.D., Indiana University Press, 1994.

Callu 1980 - J. P. Callu, The distribution and the role of bronze coinage from $A D 348$ to 392, in King, C. E. (ed.), Imperial Revenue, Expenditure and Monetary Policy in the Fourth Century AD, BAR International Series 76, 1980, 95-124.

Casey 1986 - J. Casey, Understanding ancient coins. An introduction for archaeologists and historians, Batsford, 1986

Dan 2017 - A. Dan, The Sarmatians: Some Thoughts on the Historiographical Invention of a West Iranian Migration, in Wiedemann, F., Hofmann, K. P Gehrke H. J. (eds.), Vom Wandern der Völker. Migrationserzählungen in den Altertumswissenschaften, Edition Topoi, 2017, 97-134.

Demo (ed.) 1994 - Ž. Demo (ed.), Od nepobjedivog sunca do sunca pravde - rano kršćanstvo u kontinentalnoj Hrvatskoj / From the Invincible Sun to the Sun of Justice - Early Christianity in Continental Croatia, Arheološki muzej u Zagrebu, 1994.

Duncan 1993 - G. L. Duncan, Coin Circulation in the Danubian and Balkan Provinces of the Roman Empire, 294-578, Royal Numismatic Society special publication 16, The Royal Numismatic Society, 1993.

Duncan-Jones 1994 - R. Duncan-Jones, Money and government in the Roman Empire, Cambridge University Press, 1994.

FMRD - H. J. Kellner, M. Overbeck (eds.), Die Fundmünzen der Römischen Zeit in Deutschland, Bayern / Oberpfalz, Abteilung 1/3, Römisch-Germanischen Kommission des Deutschen Archäologischen Instituts, Zabern Verlag, 1978.

FMRÖ 1/2 - F. Dick, Die Fundmünzen der Römischen Zeit in Österreich, Abteilung 1/2, Burgenland, Veröffentlichungen zur Numismatik (vormals vö der Numismatischen Kommission),Verlag der Österreichischen Akademie der Wissenschaften, 1984
FMRÖ 3/2 - M. Alram, F. Schmidt-Dick, Die Fundmünzen der römischen Zeit in Österreich. Kärnten, Band 3/2, Veröffentlichungen zur Numismatik (vormals VÖ der Numismatischen Kommission), Verlag der Österreichischen Akademie der Wissenschaften, 2007

Hanson 2005 - W. S. Hanson, Civilians on Frontiers, in Visy, Z. (ed.), Limes XIX Proceedings of the XIXth International Congress of Roman Frontier Studies held in Pécs, Hungary, September 2003, University of Pécs, 2005, 303-307.

Harris 2008 - W. V. Harris, The Nature of Roman Money, in Harris, W. V. (ed.), The Monetary Systems of the Greeks and Romans, Oxford University Press, 2008, 174-207.

Harl 1996 - K. W. Harl, Coinage in the Roman Economy zo0 B.C. to A.D. 700, The Johns Hopkins University Press, 1996.

Hendy 1985 - M. F. Hendy, Studies in the Byzantine Monetary Economy, c. 3001450, Cambridge University Press, 1985.

Howgego 2014 - C. Howgego, Questions of Coin Circulation in the Roman Period, in Dörtlük, K., Tekin, O., Boyraz Seyhan, R. (eds.), Birinci Uluslararası Anadolu Para Tarihi ve Numismatik Kongresi-Bildiriler / First International Congress of the Anatolian Monetary History and Numismatics-Proceedings, AKMED, Congress, Symposium, Seminar Series 4, 2014, 307-318.

Ilkić 2003 - M. Ilkić, Cornacum - sotinski prostor i njegovo mjesto u organizaciji južnog dijela provincije Panonije, unpublished PhD thesis, Sveučilište u Zadru, 2003.

Ilkić 2005 - M. Ilkić, Pečati na antičkim opekama i krovnim crepovima iz Sotina (Cornacum), Vjesnik Arheološkog muzeja u Zagrebu 38, 2005, 19-54.

Ilkić 2006 - M. Ilkić, Antičke plombe iz Sotina (Cornacum), Radovi Zavoda za pov ijesne znanosti HAZU u Zadru 48, 2006, 57-80.

Ilkić 2008a - M. Ilkić, Rimski Cornacum u svjetlu numizmatičkih nalaza / Roman Cornacum in the Light of the Numismatic Evidence, in Dobrinić, J. (ed.), Zbornik radova 5. međunarodnog numizmatičkog kongresa u Hrvatskoj / Proceedings of the 5th International Numismatic Congress in Croatia, Acta Numismatica, Hrvatsko numizmatičko društvo, 2008, 51-76.

Ilkić 2008b - M. Ilkić, Arheološki tragovi turnirskih igara rimske vojne posade u Kornakumu, Histria Antiqua 16, 2008, 137-144

Ilkić 2009a - M. Ilkić, Rimska vojna diploma iz Sotina (Cornacum), Priloz Instituta za arheologiju u Zagrebu 26, 2009, 143-164.

Ilkić 200gb - M. Ilkić, Rimske zoomorfne fibule iz Sotina (Cornacum), Histria an tiqua 18, 2009, 439-443.

Isaac 1988 - B. Isaac, The Meaning of the Terms Limes and Limitanei, Journal of Roman Studies 78, 1988, 125-147.

Katsari 2008 - C. Katsari, The Monetization of Rome's Frontier Provinces, in Harris, W. V. (ed.), The Monetary Systems of the Greeks and Romans, Oxford University Press, 2008, 242-266.

Kos 1986 - P. Kos, The Monetary Circulation in the South eastern Alpine Region ca. 300 B. C. - A. D. 1000, Situla - Dissertationes Musei Nationalis Sloveniae 24 Narodni Muzej Ljubljana, 1986.

Kos 2019 - P. Kos, Das spätrömische Kastell Vemania bei Isny III. Auswertung der Fundmünzen und Studien zum Münzumlauf in Raetien im 3. und 4. Jahrhundert, Münchner beiträge Zur vor- und frühgeschichte, Band 65, Bayerische Akademie der Wissenschaften, Verlag c.h.beck münchen, Munich, 2019.

Kovács 2003 - P. Kovács, The Late Roman Army, in Visy, Z. (ed.), The Roman army in Pannonia. An archaeological guide of the Ripa Pannonica, Teleki László Foundation, 2003, 31-36.

Kovács 2016 - P. Kovács, Notes on the Pannonian foederati, in Wolff, C., Faure P. (eds.), Les auxiliaires de l'armée romaine. Des alliés aux fédérés - Actes du sixième Congrès de Lyon (23-25 octobre 2014), Collection Études et Recherches sur l'Occident Romain 51, Diffusion Librairie De Boccard, 2016, 575-725.

Lo Cascio 2008 - E. Lo Cascio, The Function of Gold Coinage in the Monetary Economy of the Roman Empire, in Harris, W. V. (ed.), The Monetary Systems of the Greeks and Romans, Oxford University Press, 2008, 160-173. 
Ložnjak Dizdar, Hutinec (eds.) 2010 - D. Ložnjak Dizdar, M. Hutinec (eds.), Arheološke spoznaje o Sotinu. Rezultati probnih istraživanja 2008. - 2010., Gradski muzej Vukovar, 2010.

Ložnjak Dizdar, Hutinec 2011 - D. Ložnjak Dizdar, M. Hutinec, Sotin, probna arheološka istraživanja 2010., Annales Instituti Archaeologici 7, 2011, 9-12.

Ložnjak Dizdar, Hutinec 2013 - D. Ložnjak Dizdar, M. Hutinec, Sotin - arheološka istraživanja 2012. godine, Annales Instituti Archaeologici 9, 2013, 8-11.

Ložnjak Dizdar, Hutinec 2014 - D. Ložnjak Dizdar, M. Hutinec, Sotin - rezultati istraživanja 2013. godine, Annales Instituti Archaeologici 10, 2014, 9-13.

LRBC - R. A. G. Carson, P. V. Hill, J. P. C. Kent, Late Roman Bronze Coinage AD. 324-489, Spink, 1960.

MIR 36, 43, 44 - R. Göbl, Moneta Imperii Romani, Band 36, 43, 44, Die Münzprägung der Kaiser Valerianus I. / Gallienus / Saloninus (253/268), Regalianus (260) und Macrianus / Quietus (260/262), Veröffentlichungen der Numismatischen Kommission herausgegeben von Michael Alram, Band 35, Verlag der Österreichischen Akademie der Wissenschaften, 2000

Mirnik 1999 - I. Mirnik, Prolegomena to the numismatic topography of Burgenae, in Bertok, K., Torbagy, M. (eds.), Emlékköny Bíró-Sej Katalin és Gedai István 65. Születésnapjára/ Festschrift für Katalin Bíró-Sej und István Gedai zum 65. Geburtstag, Argumentum Kiadó, Magyar Nemzeti Múseum, Magyar Numizmatikai Társulat, 1999, 221-235.

Modéran 2008 - Y. Modéran, The Controlled Immigration of Barbarian Groups in the Roman Empire, in Aillagon, J. J. (ed.), Rome and the Barbarians: The Birth of a New World, Palazzo Grassi, 2008, 220-221.

Moorhead 2012 - S. Moorhead, The Coinage of the Later Roman Empire, 364498, in Metcalf, W. E. (ed.), The Oxford Handbook of Greek and Roman Coinage, Oxford Handbooks, Oxford University Press, 2012, 601-632.

Mócsy 1974 - A. Mócsy, Pannonia and Upper Moesia. A History of Middle Danube Provinces of the Roman Empire, History of The Provinces of The Roman Empire 4, Monographs in Social Theory, Routledge \& K. Paul, 1974.

Nađ 2012 - M. Nađ, Coin Circulation 193-423, in Migotti, B. (ed.), The Archaeology of Roman Southern Pannonia, The state of research and selected problems in the Croatian part of the Roman province of Pannonia, BAR International Series 2393, Archaeopress, 2012, 385-409.

Nađ 2012 - M. Nađ, Coin hoards in Croatia - An update on the CHY, Vjesnik Arheološkog muzeja u Zagrebu 45, 2012, 395-466.

Pinterović 2014 - D. Pinterović, Mursa, Mursa Aeterna 1, Hrvatska akademija znanosti i umjetnosti, Zavod za znanstveni i umjetnički rad u Osijeku, Muzej Slavonije, 2014

Radman-Livaja 2005 - I. Radman-Livaja, Finds of Roman Military Equipment from Teutoburgium, in Visy, Z. (ed.) 2005, Limes XIX: Proceedings of the XIXth International Congress of Roman Frontier Studies held in Pécs, Hungary, September 2003, University of Pécs, 2005, 939-952.

Radman-Livaja 2012 - I. Radman-Livaja, The Roman Army, in Migotti (ed.) 2012, The Archaeology of Roman Southern Pannonia, The state of research and selected problems in the Croatian part of the Roman province of Pannonia, BAR International Series 2393, Archaeopress, 2012, 159-189.

RAMMU 1-3 - P. Prohászka, M. Torbágyi, Regesten der antiken Münzfunden und Münzhorte in Ungarn 1-3, Martin Opitz Verlag, 2017.

Rankov Kondić 2013 - J. Rankov Kondić, Moesian Late Roman limes on the Danube Region of the Iron Gates/ Đerdap, in Popović, I., Borić-Brešković, B. (eds.), Constantine the Great and the Edict of Milan 313. The Birth of Cristianity in the Roman Provinces on the Soil of Serbia, Archaeological monographs 22, Narodni muzej u Beogradu, 2013, 36-59.
Ravetz 1964 - A. Ravetz, The fourth century inflation and romano-british coin finds, The Numismatic Chronicle and Journal of the Royal Numismatic Society 4. 1964, 201-231.

Reece 1977 - R. Reece, Frontiers, Army, Coins, in Fitz, J. (ed.), Limes: Akten des XI. Internationalen Limeskongresses, Akadémiai Kiadó, 1977, 643-646.

Reece 1982 - R. Reece, Economic History from Roman site-finds, in Hacken, T., Weiller, R. (eds.), Actes du gème Congrès international de numismatique / Proceedings of the gth International Congress of Numismatics, Association internationale des numismates professionnels, Association internationale des numismates professionnels, 1982, 495-502.

Reece 1984 - R. Reece, Mints, markets and the military, in Blagg, T.F.C., King, A.C. (eds.) 1984, Military and Civilian in Roman Britain. Cultural Relationships in a Frontier Province, BAR 136, 1984, 143-160.

Reece 2003 - R. Reece, Coins and the Late Roman Economy, in Lavan, L., Bowden, W. (eds.), Theory and practice in Late Roman Archaeology, Brill, 2003, 139-168.

Reece 2016 - R. Reece, Categories of coin finds, in Asolati, M., Callegher, B.Saccocci, A. (eds.), Suadente nummo vetere. Studi in onore di Giovanni Gorini, Esedra, 2016, 107-125

RIC 9 - J. W. E. Pearce, Roman Imperial Coinage 9, Valentinian I - Theodosius I, Spink \& Son, Ltd., 1968.

RIC 10 - J. P. C. Kent, Roman Imperial Coinage 10, The divided empire and the fall of the western parts, AD 395-491, Spink \& Son, Ltd., 1994.

Sanader 2003 - M. Sanader, The ripa Pannonica in Croatia, in Visy, Z. (ed.), The Roman army in Pannonia. An archaeological guide of the Ripa Pannonica, Teleki László Foundation, 2003, 135-142.

Sanader 2010 - M. Sanader, On the problem of topography of the Croatian part of the Danube limes based on recent archaeological excavations, in RadmanLivaja, I. (ed.), Nalazi rimske vojne opreme u Hrvatskoj / Finds of the Roman military equipment in Croatia, Arheološki muzej u Zagrebu, 2010, 221-231.

Torbágy 2000 - M. Torbágy, Osvrt na kolanje rimskog novca u Panoniji nakon 375. godine, Zbornik radova 2. Međunarodog numizmatičkog kongresa u Hrvatskoj, 15.-17. listopada 1998., Opatija, Numizmatički studio Dobrinić \& Dobrinić, 2000, 43-56.

TP - R. Talbert, The Peutinger Map, Digital publication on the website of Cambridge University Press, 2010.

Visy 2003 - Z. Visy, The Limes Road Along the Danube, in Visy, Z. (ed.) 2003, The Roman army in Pannonia. An archaeological guide of the Ripa Pannonica, Teleki László Foundation, 2003, 43-46.

Vojvoda, Mrđić 2017 - M. Vojvoda, N. Mrđić, Nalazi novca sa viminacijumske nekropole Pećine i njihova uloga u pogrebnom ritual / Coin finds from the Viminacium necropolis of Pećine and their role in funerary ritual, VIMINACIVM 5, Arheološki institut Beograd, 2017.

Vojvoda, Redžić 2018 - M. Vojvoda, S. Redžić, Циркулација римског новца у српском делу лимеса (I-V век) / Circulation of Roman coins in the Serbian part of the limes (1st-5th century,) in Korać, M., Pop-Lazić, S. (eds.), Римски лимес и градови на тлу Србийе / Roman Limes and Cities on the Territory of Serbia, Српска академија наука и уметности, Археолошки институт Беозрад, 2018, 50-56.

Vučić 2013 - J. Vučić, Optjecaj novca u kasnoantičkom Zadru / Coin circulation in Zadar in the Late Roman period, Archaeologia Adriatica 7, 2013, 221-269.

Vukmanić 2009 - I. Vukmanić, Granice Rimskog Carstva - spomenik svjetske baštine, Osječki zbornik 29, 2009, 25-36. 